\title{
String Theory: exact solutions, marginal deformations and hyperbolic spaces
}

Domenico Orlando 

A Lei 


\section{Contents}

$\begin{array}{llr}1 & \text { Introduction } & 1\end{array}$

2 Wess-Zumino-Witten Models $\quad \mathbf{5}$

Wess-Zumino-Witten models constitute a large class of the exact string theory solutions which we will use as starting points for most of the analysis in the following. In this chapter we see how they can be studied from different perspectives and with different motivations both from a target space and world-sheet point of view.

2.1 The two-dimensional point of view . . . . . . . . . . 5

2.2 The target space point of view . . . . . . . . . . . . 14

\section{Deformations}

In this rather technical chapter we describe marginal deformations of Wess-Zumino-Witten models. The main purpose for these constructions is to reduce the symmetry of the system while keeping the integrability properties intact, trying to preserve as many nice geometric properties as possible.

3.1 Deformed WZW models: various perspectives . . . . . . . . . 18

3.2 Background fields for the asymmetric deformation . . . . . . . 22

3.3 Geometry of squashed groups . . . . . . . . . . . . . . . 26

3.4 A no-renormalization theorem . . . . . . . . . . . . . 29

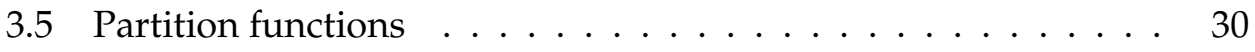

3.6 The deformation as a gauging $\ldots \ldots \ldots \ldots$

\section{Applications}

In this chapter we present some of the applications for the construction outlined above. After an analysis of the most simple (compact and noncompact) examples, we describe the near-horizon geometry for the BertottiRobinson black hole, show some new compactifications and see how Horne and Horowitz's black string can be described in this framework and generalized via the introduction of an electric field.

4.1 The two-sphere CFT . . . . . . . . . . . . . . . . . . . . 43

$4.2 S L(2, \mathbb{R}) \ldots \ldots \ldots \ldots \ldots \ldots \ldots \ldots \ldots$

4.3 Near horizon geometry for the Bertotti-Robinson black hole . . 59

4.4 The three-dimensional black string revisited . . . . . . . . . 62

4.5 New compactifications . . . . . . . . . . . . . 76 
5 Squashed groups in type II

In this chapter we start deviating from the preceding ones because we will no longer deal with WZW models but with configurations in which the group manifold geometry is sustained by RR fields. In particular, then, we see how the squashed geometries can be obtained in type II theories by Tdualizing black brane configurations.

$5.1 S L(2, \mathbb{R}) \times S U(2)$ as a D-brane solution . . . . . . . . . . 85

5.2 T duality with RR fields . . . . . . . . . . . . . . . . . . . 86

5.3 The squashed sphere $\ldots \ldots \ldots . \ldots . \ldots 88$

6 Out of the conformal point: Renormalization Group Flows 91

This chapter is devoted to the study of the relaxation of squashed WZW models further deformed by the insertion of non-marginal operators. The calculation is carried from both the target space and world-sheet points of view, once more highlighting the interplay between the two complementary descriptions. In the last part such techniques are used to outline the connection between the time evolution and the RG-flow which is seen as a large friction limit description; we are hence naturally led to a FRW-type cosmological model.

6.1 The target space point of view . . . . . . . . . . . . . . 92

6.2 The CFT approach . . . . . . . . . . . . . . . . . . 102

6.3 RG flow and friction . . . . . . . . . . . . . . . 105

6.4 Cosmological interpretation . . . . . . . . . . . . . . 110

7 Hyperbolic Spaces $\quad 115$

In this chapter we investigate type II and M-theory geometries written as direct products of constant-curvature spaces. We find in particular a class of backgrounds with hyperbolic components and we study their stability with respect to small fluctuations.

7.1 M-theory solutions . . . . . . . . . . . . . . . 115

7.2 Stability . . . . . . . . . . . . . . . . . . 119

7.3 Type IIB backgrounds . . . . . . . . . . . . . . . . . . . . . . 128

8 Conclusions and further perspectives $\quad 133$

$\begin{array}{ll}\text { A Table of conventions } & 135\end{array}$

B Explict parametrizations for some Lie groups $\quad 137$

B.1 The three-sphere . . . . . . . . . . . . . . . . 137

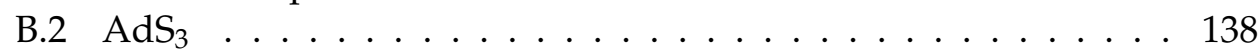

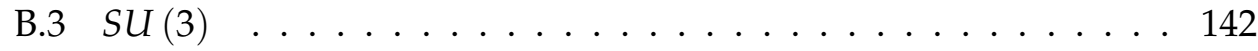

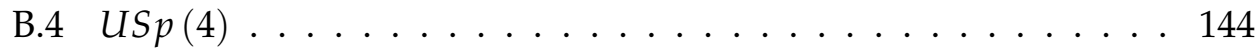

$\begin{array}{ll}\text { C Symmetric deformations of } S L(2, \mathbb{R}) & 147\end{array}$

D Spectrum of the $S L(2, \mathbb{R})$ super-WZW model 149

$\begin{array}{ll}\text { Bibliography } & 153\end{array}$ 


\title{
Acknowledgements
}

\author{
Never had nay man a wit so \\ presently excellent, as that it \\ could raise itselfe; but there must \\ come both matter, occasion, \\ commenders, and favourers to it.
}

Il Volpone BEN JOHNSON

While writing these acknowledgements I realized how much I'm indebted to so many people. I learned a lot from them all - not just physics - and without them this work simply wouldn't have been possible. I'm pretty sure I'm forgetting someone. I plead guilty. But even if you don't find your name rest assured that you'll always have my personal gratitude.

In any case, I simply can't start without mentioning my parents for - although far - they've always been present in their peculiar way.

I must thank the members of my thesis committee: Luis Alvarez-Gaumé, Edouard Brezin, Costas Kounnas, Marios Petropoulos, Alex Sevrin, Philippe Spindel, Arkady Tseytlin for they extremely precious feedback. They turned my defence into a valuable learning experience.

I wish to thank Patrick Mora for having welcomed me as a $\mathrm{PhD}$ student at the Centre de physique théorique of the École polytechnique. Also I thank Edouard Brezin at École normale supèrieure, Dieter Lüst at Max Planck Institut (Munich) and Alex Sevrin at Vrije Universiteit Brussel. During the last year they hosted me for several months at their respective institutions. Each and all of them have made of my stays invaluable experiences from both the professional and human points of view.

Then I thank my advisor Marios Petropoulos for all what he taught me and for his continuous support. In these years I learned to think of him as a friend. Costas Kounnas for letting me camp in his office at ENS where he would devote me hours and hours of his time, sharing his experience and his amazing physical intuition. Costas Bachas, who was among the first professors I had when I arrived in Paris and (unknowingly) pushed me towards string theory. Since then he helped me in many an occasion and I'm almost embarrassed if I think how much I learned from him and in general I owe him.

Doing one's PhD in Paris is a great opportunity for there are not so many places in the world with such a physics community. And it's definitely surpris- 
ing to a PhD student, like I was, how everybody is always willing to discuss and makes you feel one of them. I learned a lot from many people and I hope that those I don't remember explicitly won't take it too bad. On the other hand I couldn't not mention Adel Bilal, Eugène Cremmer, Pierre Fayet, Mariana Graña, Jean Iliopoulos, Bernard Julia, Volodia Kazakov, Ruben Minassian, Hervé Partouche, Michela Petrini, Boris Pioline, Ian Troost, Pierre Vanhove.

Sometimes discussions would lead to a publication and I'm honored to count among my collaborators Costas Bachas, Ioannis Bakas, Davide Cassani, Stéphane Detournay, Dan Israël, Marios Petropoulos, Susanne Reffert, Kostas Sfetsos, Philippe Spindel. I really wish to thank you for what I learned and keep learning from you all.

I must remember my office mates Luciano Abreu, Stéphane Afchain, Yacine Dolivet, Stéphane Fidanza, Pascal Grange, Claudia Isola, Liguori Jego, Jerome Levie, Xavier Lacroze, Alexey Lokhov, Liuba Mazzanti, Hervé Moutarde, Chloé Papineau, Susanne Reffert, Sylvain Ribault, Barbara Tumpach and especially Claude de Calan. With all of you I shared more than an office and our never-ending discussions have really been among the most precious moments of my $\mathrm{PhD}$. I hope none of you will get mad at me if in particular I thank Sylvain for all the advice he gave me, even before starting my PhD. Not to speak of my brother Pascal.

A final thanks (last but not least) goes to the computer people and secretaries at CPHT and ENS, without whom life couldn't be that easy.

Thanks, all of you. 


\title{
CHAPTER 1
}

\section{Introduction}

\author{
There is a theory which states that if ever \\ anyone discovers exactly what the \\ Universe is for and why it is here, it will \\ instantly disappear and be replaced by \\ something even more bizarre and \\ inexplicable
}

The Restaurant at the End of the Universe

$\mathrm{I}^{\mathrm{N}}$ THE LAST FIFTY YEARS theoretical physics has been dominated by two apparently incompatible models: the microscopic world being described by quantum field theory and the macroscopic word by general relativity. QFT is by far the most successful theory ever made, allowing to reach an almost incredible level of accuracy in its measurable predictions. But gravity is different from all other interactions; although by far the weakest, it acts on the very structure of the universe at a more fundamental level. Many attempts have been made to obtain a consistent quantum theory of gravity and they all proved unsuccessful so that it is has become clear that completely new ideas are needed.

To this day, but this has been true for more than twenty years now, the only promising trail we can follow in the quest for this unification is string theory. Roughly speaking it postulates that the fundamental objects are not point particles as in the standard quantum models but one-dimensional objects strings. Although their typical size is so small that one might even question the very meaning of distance at this scale, the mere not being pointlike allows to solve an enormous number of theoretical problems and in particular those connected with the severe divergencies that gravity presents due to the local nature of interactions. Field theory particles appear as vibration modes of the fundamental string, spacetime is a semiclassical description for a string condensate, supergravity emerges as a low-energy limit, and the standard model is the result of a compactification in presence of extended objects ( $\mathrm{D}$ branes).

Of course this is in many ways a wish-list. In its present state string theory is largely incomplete. To begin, only a first-quantization description is known 
and as such is intrinsically perturbative, and only the S-matrix elements in a given vacuum are accessible. So, although in principle the very geometry should emerge from string dynamics, in practice we are forced to choose a vacuum, which by itself clips the wings of any hope of attaining a complete quantum gravity theory. Moreover only the perturbative regime is in principle available, even though the existence of a web of dualities can translate strong coupling backgrounds into ones we can deal with.

One thing nevertheless must be kept in mind. One should stay as close as possible to the present knowledge of Nature and try to predict the outcomes of realistic experiments beyond the standard models in particle physics or cosmology by using phenomenomogical models, but string theory is not supergravity. It is reassuring to find it as a low energy limit but most, if not all, of the new physics lies in the regime where the semiclassical approximations break down. From this point of view an important, almost essential, rôle is played by exact models, i.e. systems in which the $\alpha^{\prime}$ corrections can be kept under control and a conformal field theory description is possible. Because of technical issues, not many such backgrounds are known and they are all characterized by a high degree of symmetry. Hence it is not surprising that they in general do not have a direct phenomenological impact. But the reason for their fundamental importance lies elsewhere. They can mostly be used as laboratories to study the extremal conditions - black hole dynamics just to name one - in which general relativity and field theory show their limits. The very reason why string theory was introduced.

Plan of the thesis This thesis is almost entirely devoted to studying string theory backgrounds characterized by simple geometrical and integrability properties. This requires at the same time a good grasp on both the low-energy (supergravity) description in terms of spacetime and on the CFT side controlling all-order-in- $\alpha^{\prime}$ effects.

The archetype of this type of system is given by Wess-Zumino-Witten models, describing string propagation in a group manifold or, equivalently, a class of conformal field theories with current algebras. Given their prominent rôle we devote the whole Chapter 2 to recall their properties from different points of view, trying to outline some of the most important features.

In Chapter 3 we enter the main subject of these notes, namely we study the moduli space of WZW models by using truly marginal deformations obtained as bilinears in the currents. A vast literature exists on this type of constructions, but we will concentrate on a particular class, which we dub asymmetric deformations. They actually present a number of advantages over the more familiar symmetric ones and in particular, although the CFT description is slightly more involved (Sec. 3.5), they enjoy a very nice spacetime interpretation. This can be completely understood in terms of the always-underlying Lie algebra (Sec. 3.3) and can be proven to remain unchanged at all orders in $\alpha^{\prime}$ (Sec. 3.4).

The following Chapter 4 illustrates some of the obvious applications for our construction. We then start with the simplest $S U(2)$ case, leading to a 
CFT on the squashed three-sphere and on the two-sphere (Sec. 4.1). Then we deal with the considerably richer non compact $S L(2, \mathbb{R})$ case (Sec. 4.2). This leads naturally to the description of some black hole geometries such as the near-horizon limit for the Bertotti-Robinson black hole (Sec. 4.3), and the three-dimensional electrically charged black string (Sec. 4.4). Both can be studied in terms of CFT, thus allowing for an evaluation of the spectrum of string primaries. Further applications regard the possibility of introducing new compactification manifolds as part of larger, ten-dimensional backgrounds (Sec. 4.5).

In Chapter 5 we consider an alternative description for the squashed group geometries which are found to be T-duals of the usual type II black brane solutions.

In Chapter 6 we take a slight detour from what we have seen up to this point: instead of exact CFT backgrounds we deal with off-shell systems. Using a renormalization-group approach we describe the relaxation towards the symmetrical equilibrium situation. This same behaviour is studied from different points of view, RG flow in target space (Sec. 6.1), two-dimensional renormalization (Sec. 6.2) and reading the flow as a motion in an extra time direction (Sec. 6.3), thus obtaining Freedman-Robertson-Walker-like metrics that in the most simple case describe an isotropic universe with positive cosmological constant undergoing a big-bang-like expansion (Sec. 6.4).

The final Chapter 7 marks a further deviation from the construction of exact models: we consider in fact backgrounds with Ramond-Ramond fields which still elude a satisfactory CFT interpretation. In particular we analyze direct products of constant-curvature spaces and find solutions with hyperbolic spaces sustained by RR fields.

The themes we treat here have been the subject of the following publications:

- D. Israël, C. Kounnas, D. Orlando and P. M. Petropoulos, Electric / magnetic deformations of $S^{* *} 3$ and $A d S(3)$, and geometric cosets, Fortsch. Phys. 53, 73-104 (2005), hep-th/0405213.

- D. Israel, C. Kounnas, D. Orlando and P. M. Petropoulos, Heterotic strings on homogeneous spaces, Fortsch. Phys. 53, 1030-1071 (2005), hep-th/0412220.

- D. Orlando, $A d S(2) \times S^{* * 2}$ as an exact heterotic string background, (2005), hep-th/0502213, Talk given at NATO Advanced Study Institute and EC Summer School on String Theory: From Gauge Interactions to Cosmology, Cargese, France, 7-19 Jun 2004.

- S. Detournay, D. Orlando, P. M. Petropoulos and P. Spindel, Threedimensional black holes from deformed anti de Sitter, JHEP 07, 072 (2005), hep-th/0504231.

- D. Orlando, Coset models and D-branes in group manifolds, (2005), hep-th/0511210. Published in Phys.Lett.B 
- D. Orlando, P. M. Petropoulos and K. Sfetsos, Renormalization-group flows and charge transmutation in string theory, Fortsch. Phys. 54, 453-461 (2006), hep-th/0512086.

Some results are on the other hand still unpublished. This concerns in particular Chapter 5 and the second half of Chapter 6 and Chapter 7. 


\section{CHAPTER 2}

\section{Wess-Zumino-Witten Models}

Wess-Zumino-Witten models constitute a large class of the exact string theory solutions which we will use as starting points for most of the analysis in the following. In this chapter we see how they can be studied from different perspectives and with different motivations both from a target space and world-sheet point of view.

“To paraphrase Oedipus, Hamlet, Lear, and all those guys," I said, "I wish I had known this some time ago."

Amber Chronicles

ROGER ZELAZNY

\subsection{The two-dimensional point of view}

\section{The classical theory}

$W_{\text {paper [Wit84] to generalize the usual bosonization of a free fermion to }}^{\text {ESS-ZuMinO-WITTEN models were introduced by Witten in his seminal }}$ a system of $N$ fermions. It has been known for a long time that the Lagrangian for a free massless Dirac fermion in two dimensions can be mapped to the Lagrangian for a free massless boson as follows:

Free fermion

$$
L=\psi^{*} \imath \partial \psi \rightarrow \frac{1}{2} \partial_{\mu} \phi \partial^{\mu} \phi,
$$

but the generalization to more complex systems is not straightforward. One of the main motivations for this mapping is given by the fact that bosonic systems admit a semiclassical limit and then allow for simpler intuitive interpretations of their physics.

It is of course possible to rewrite the fields in one description as functions of the fields in the other one but this requires complicated (non-local) expressions. Other quantities remain simple, in particular the currents:

$$
J_{\mu}=\psi^{*} \gamma_{\mu} \psi \rightarrow \frac{1}{\sqrt{\pi}} \epsilon_{\mu \nu} \partial^{v} \phi
$$


and as such they are the convenient building blocks for a generalization. A most useful rewriting for their expression is obtained when going to light-cone coordinates

$$
J=\frac{i}{2 \pi} U^{-1} \partial U=-\frac{1}{\sqrt{\pi}} \partial \phi, \quad \bar{J}=-\frac{i}{2 \pi} \bar{\partial} U U^{-1}=\frac{1}{\sqrt{\pi}} \bar{\partial} \phi,
$$

$U=\exp [i \sqrt{4 \pi} \phi]$ being the chiral density $\bar{\psi}\left(1+\gamma_{5}\right) \psi$. One then finds that the currents are chirally conserved:

$$
\partial \bar{J}=\bar{\partial} J=0,
$$

which is equivalent to ask for the bosonic field to be harmonic:

$$
2 \partial \bar{\partial} \phi=0 .
$$

The generalization of this simple system is given by the theory of $2 \mathrm{~N}$ Dirac fermions.

$$
L=\frac{1}{2} \sum_{k} \bar{\psi}^{k} i \not \partial \psi^{k}
$$

This admits a chiral group $U(N) \times U(N)$ with vector and axial currents written as:

$$
V_{\mu}^{a}=\bar{\psi} \gamma_{\mu} T^{a} \psi, \quad A_{\mu}^{a}=\bar{\psi} \gamma_{\mu} \gamma_{5} T^{a} \psi
$$

It is more useful to define the chiral components

$$
J^{i j}=-i \psi^{i} \psi^{j}, \quad \bar{J}^{i j}=-i \bar{\psi}^{i} \bar{\psi}^{j},
$$

generating the $U(N) \times U(N)$ symmetry and obeying the same conservation as before:

$$
\partial \bar{J}=\bar{\partial} J=0,
$$

This is obviously not equivalent to a system of $N$ bosons which would just allow for the diagonal $U(1)^{N}$ symmetry. What we need is an object $g \in U(N)$ transforming under a couple $(A, B) \in U(N) \times U(N)$ as

$$
g \rightarrow A g B^{-1}, A, B \in U(N)
$$

and express the currents as functions of $g$ just as in the Abelian case we did in terms of the density $U$ :

$$
J=\frac{i}{2 \pi} g^{-1} \partial g, \quad \quad \bar{J}=-\frac{i}{2 \pi} \bar{\partial} g g^{-1} .
$$

In order for these currents to be conserved as above all we need to find is a Lagrangian admitting the following equations of motion

$$
\partial\left(g^{-1} \bar{\partial} g\right)=0 .
$$


The first natural tentative action is given by

$$
S_{0}=\frac{1}{4 \lambda^{2}} \int_{\Sigma} \mathrm{d}^{2} x \operatorname{tr}\left[\partial^{\mu} g \partial_{\mu} g^{-1}\right]
$$

since this is the only manifestly chirally invariant choice. Unfortunately this can't be the right answer for a number of reasons. In particular it describes an asymptotically free theory with the wrong equations of motion. We are then forced to add another ingredient, the so-called Wess-Zumino term

$$
\Gamma=\frac{1}{24 \pi} \int_{M} d^{3} y \epsilon^{\mu v \rho} \operatorname{tr}\left[\tilde{g}^{-1} \partial_{\mu} \tilde{g} \tilde{g}^{-1} \partial_{\nu} \tilde{g} \tilde{g}^{-1} \partial_{\rho} \tilde{g}\right]
$$

where $M$ is a three-dimensional manifold admitting $\Sigma$ as border $\partial M=\Sigma$ and $\tilde{g}$ is the extension of the mapping $g: \Sigma \rightarrow G$ to a mapping $\tilde{g}: M \rightarrow G$. Although it might appear a bit surprising at first sight, this is precisely what is needed since the variation of $\Gamma$ gives only a local term on $\Sigma$ and the equations of motion for the action $S=S_{0}+k \Gamma$ read:

$$
\left(\frac{1}{2 \lambda^{2}}+\frac{k}{8 \pi}\right) \partial\left(g^{-1} \bar{\partial} g\right)+\left(\frac{1}{2 \lambda^{2}}-\frac{k}{8 \pi}\right) \bar{\partial}\left(g^{-1} \partial g\right)=0,
$$

which in particular for $\lambda^{2}=\frac{4 \pi}{k}$ yield precisely the equations we were expecting. It can be shown that this is an infrared fixed point for a renormalization group-flow, and we will expand on this aspect in Ch. 6 .

At this point it is on the other hand better to deal more thoughtfully with the interpretation and the consequences of the Wess-Zumino term $\Gamma$. First of all it must be remarked that $\Gamma$ can be put in the form of an ordinary action $i e$ an integral over the two-dimensional space-time of a perfectly respectable although non-manifestly chiral-invariant action (which changes by a total derivative under a chiral transformation):

$$
\Gamma=\int_{\Sigma} d^{2} x \epsilon^{\mu v} B_{i j}\left(\phi^{k}\right) \partial_{\mu} \phi^{i} \partial_{\nu} \phi^{j}
$$

where $B$ is a (Kalb-Ramond) two-form. Another important aspect is the fact that an ambiguity is present in the definition of $\Gamma$ for there are infinite topologically inequivalent ways to extend $g$ to $\tilde{g}$, classified by $\pi_{3}(G)$. In the case of a compact group $\pi_{3}(G)=\mathbb{Z}$ and we are led to the same kind of topological argument leading to the quantization of the Dirac monopole. In fact if we consider a different three-manifold $M^{\prime}$ in the definition of $\Gamma$, the string amplitude changes by

$$
\exp \left[\imath \int_{M} H-\imath \int_{M^{\prime}} H\right],
$$

where $H=\mathrm{d} B$. This implies that the theory is consistent only if

$$
\frac{1}{2 \pi} \int_{S^{3}} H \in \mathbb{Z}
$$


having remarked that $M$ and $M^{\prime}$ have the same boundary and then $M-M^{\prime} \sim$ $S^{3}$. Using the normalizations above one can show that this is equivalent to asking $k \in \mathbb{Z}$ which can be read as a quantization condition on the radius of the group manifold. In particular, the semiclassical limit is obtained when $k \rightarrow \infty$.

For reasons that will appear evident in the following $k$ is called level of the model and the action is written as:

$$
S_{k}[g]=\frac{k}{16 \pi} \int_{\Sigma} \mathrm{d}^{2} z\left\langle g^{-1} \partial g, g^{-1} \bar{\partial} g\right\rangle+\frac{k}{24 \pi} \int_{M}\left\langle\tilde{g}^{-1} \mathrm{~d} \tilde{g},\left[\tilde{g}^{-1} \mathrm{~d} \tilde{g}, \tilde{g}^{-1} \mathrm{~d} \tilde{g}\right]\right\rangle,
$$

where $\langle\cdot, \cdot\rangle$ is the Killing form on $G$.

\section{An exact model}

An important feature of WZW models is that they provide exact solutions at all orders in $\alpha^{\prime}$ or, more precisely, the effective action is equal to the classical ac-

Quantum effective action for WZW models

tion up to a shift in the overall normalization $k \rightarrow k+g^{*}$. The argument goes as follows [KZ84, LS92, Tse93]. Consider the path integral

$$
\int \mathscr{D} g e^{-k S[g]+B \bar{J}[g]}=e^{-W[B]}
$$

where $S[g]$ is the WZW action at level one and $B \bar{J}[g]$ is the shorthand for

$$
B \bar{J}[g]=\frac{k}{\pi} \int \mathrm{d} z^{2}\left\langle B \bar{\partial} g g^{-1}\right\rangle .
$$

If we write $B$ as $B=u^{-1} \partial u$ we can use the so called Polyakov-Witten identity

$$
S[a b]=S[a]+S[b]-\frac{1}{\pi} \int \mathrm{d}^{2} z\left\langle a^{-1} \partial a \bar{\partial} b b^{-1}\right\rangle
$$

and it's then easy to see that $W$ doesn't receive quantum corrections and is simply given by the classical action evaluated on $u$ :

$$
W[B]=-k S[u] .
$$

Indeed, $B$ is an external source coupled with the current $\bar{J}$ so, the effective action for $g, \Gamma[g]$, will be given by the Legendre transformation of $W[B]$, ie by the path integral:

$$
\int \mathscr{D} B e^{-W[B]+B \bar{J}[g]}=e^{-\Gamma[g]} .
$$

This interpretation of effective action for $\Gamma[g]$ is comforted by remarking that combining Eq. (2.20) and Eq. (2.24) one finds that

$$
e^{-\Gamma[g]}=\int \mathscr{D} g^{\prime} e^{-k S\left[g^{\prime}\right]} \delta\left(\bar{J}\left[g^{\prime}\right]-\bar{J}[g]\right) .
$$

In order to calculate the Legendre transform in Eq. (2.24) one can perform a change of variables from $B=u^{-1} \partial u$ to $u$ : the corresponding Jacobian will give 
the announced shift in the effective action. More precisely, as shown in [PW83, Pol88] we have:

$$
\frac{\mathscr{D} B}{\mathscr{D} u}=e^{g^{*} S[u]}
$$

and putting this back in Eq. (2.24)

$$
e^{-\Gamma[g]}=\int \mathscr{D} u e^{\left(k+g^{*}\right) S[u]+B[u] \bar{J}[g]},
$$

we can use the same technique as above to derive the celebrated result:

$$
\Gamma[g]=\left(k+g^{*}\right) S[g]
$$

\section{The CFT approach}

\section{CFT with current algebras}

Let us forget for a moment the WZW models and consider a more general framework, ie two-dimensional conformal field theories with current algebras.

CFT infinitesimal generators Given the stress-energy tensor $T_{\mu \nu}(\zeta)$ in two dimensions one can define

$$
\begin{aligned}
& T=T_{11}-T_{22}+2 \imath T_{12} \\
& \bar{T}=T_{11}-T_{22}-2 \imath T_{12}
\end{aligned}
$$

so that the conservation $\partial_{\mu} T^{\mu \nu}=0$ and the zero trace condition $T^{\mu}{ }_{\mu}=0$ translate into analyticity conditions

$$
\bar{\partial} T=\partial \bar{T}=0 .
$$

It is then clear that since the stress-energy tensor is the infinitesimal generator for translations, $T$ and $\bar{T}$ have this role for the conformal transformations.

$$
\begin{aligned}
& z \rightarrow z+\epsilon(z) \\
& \bar{z} \rightarrow \bar{z}+\bar{\epsilon}(\bar{z})
\end{aligned}
$$

which is to say that if we take a local field $A(z, \bar{z})$, this transforms under such variations as

$$
\delta_{\epsilon} A(z, \bar{z})=\oint_{z} T(\zeta) \epsilon(\zeta) A(z, \bar{z}) \mathrm{d} \zeta
$$

where the contour integral is around $z^{1}$.

This is just the definition of two-dimensional CFT but if the theory is at the same time invariant under a $G(z) \times G(\bar{z})$ action, $G$ being some Lie group, then there are additional generators $J(z)$ and $\bar{J}(\bar{z})$ allowing to express the variation of $A(z, \bar{z})$ as

$$
\delta_{\omega} A(z, \bar{z})=\oint_{z} J^{a}(\zeta) \omega^{a}(\zeta) A(z, \bar{z}) \mathrm{d} \zeta
$$


where $\omega(z)$ is some element in Lie $G$ (the algebra as usual parametrizes the infinitesimal group transformations).

The variations of those generators with respect to $\epsilon$ and $\omega$ stem from general principles and read

$$
\begin{aligned}
\delta_{\epsilon} T(z) & =\epsilon(z) T^{\prime}(z)+2 \epsilon^{\prime}(z) T(z)+\frac{1}{12} c \epsilon^{\prime \prime}(z) \\
\delta_{\epsilon} J(z) & =\epsilon(z) J^{\prime}(z)+\epsilon^{\prime}(z) J(z) \\
\delta_{\omega} J^{a}(z) & =f_{b c}^{a} \omega^{b}(z) J^{c}(z)+\frac{1}{2} k \omega^{a \prime}(z)
\end{aligned}
$$

This is just a way of writing the algebra of the generators. Using the definition above in terms of contour integrals it can also be put in terms of operator product expansions

$$
\begin{aligned}
T(z) T(w) & =\frac{c}{2(z-w)^{4}}+\frac{2}{(z-w)^{2}} T(w)+\frac{1}{z-w} T^{\prime}(w) \\
T(z) J(w) & =\frac{1}{(z-w)^{2}} J(w)+\frac{1}{z-w} J^{\prime}(w) \\
J^{a}(z) J^{b}(w) & =\frac{k \delta^{a b}}{(z-w)^{2}}+\frac{f_{c}^{a b}}{z-w} J^{c}(w)
\end{aligned}
$$

Any operator in the theory is characterized by a representation for the left and right $G$ groups and its anomalous dimensions $\Delta$ and $\bar{\Delta}$, which depend on the behaviour of the operator under dilatation $z \rightarrow \lambda z$. More precisely an operator of weight $(\Delta, \bar{\Delta})$ transforms under $z \rightarrow \lambda z, \bar{z} \rightarrow \bar{\lambda} \bar{z}$ as $A \rightarrow \lambda^{\Delta} \bar{\lambda}^{\bar{\Delta}} A$, and in particular the primary fields are defined as those who satisfy

$$
\begin{aligned}
T(z) \phi(w, \bar{w}) & =\frac{\Delta}{(z-w)^{2}} \phi(w, \bar{w})+\frac{1}{z-w} \frac{\partial}{\partial w} \phi(w, \bar{w}) \\
J^{a}(z) \phi(w, \bar{w}) & =\frac{t^{a}}{z-w} \phi(w, \bar{w}) .
\end{aligned}
$$

Given these relations it is immediate to write the Ward identities satisfied by the correlation functions of primary fields:

$$
\begin{aligned}
\left\langle T(z) \phi_{1}\left(z_{1}, \bar{z}_{1}\right) \ldots \phi_{N}\left(z_{N}, \bar{z}_{N}\right)\right\rangle= & \sum_{j=1}^{N}\left(\frac{\Delta_{j}}{\left(z-z_{j}\right)^{2}}+\frac{1}{z-z_{j}} \frac{\partial}{\partial z_{j}}\right) \times \\
& \times\left\langle\phi_{1}\left(z_{1}, \bar{z}_{1}\right) \ldots \phi_{N}\left(z_{N}, \bar{z}_{N}\right)\right\rangle \\
\left\langle J^{a}(z) \phi_{1}\left(z_{1}, \bar{z}_{1}\right) \ldots \phi_{N}\left(z_{N}, \bar{z}_{N}\right)\right\rangle= & \sum_{j=1}^{N} \frac{t_{j}^{a}}{z-z_{j}}\left\langle\phi_{1}\left(z_{1}, \bar{z}_{1}\right) \ldots \phi_{N}\left(z_{N}, \bar{z}_{N}\right)\right\rangle
\end{aligned}
$$

\footnotetext{
${ }^{1}$ In the following we will avoid to write the anti-holomorphic counterpart for each relation since it can always be trivially derived.
} 
A further step can be made if one expands the operators $T$ and $J$ in Laurent series obtaining respectively the modes $L_{n}$ and $J_{n}$ which by definition act on a local operator to give

$$
\begin{aligned}
L_{n} A(z, \bar{z}) & =\oint_{z} T(\zeta)(\zeta-z)^{n+1} A(z, \bar{z}) \mathrm{d} \zeta \\
J_{n}^{a} A(z, \bar{z}) & =\oint_{z} J^{a}(\zeta)(\zeta-z)^{n+1} t^{a} A(z, \bar{z}) \mathrm{d} \zeta
\end{aligned}
$$

and in particular the primaries will satisfy

$$
\begin{array}{lrl}
L_{n} \phi & =J_{n}^{a} \phi=0 & \forall n>0 \\
L_{0} \phi & =\Delta \phi & J_{0}^{a} \phi=t^{a} \phi
\end{array}
$$

The commutation relations among the $L_{n}$ 's and the $J_{n}^{a \prime}$ s are natural consequences of the OPEs in Eq. (2.35) and read

$$
\begin{aligned}
{\left[L_{n}, L_{m}\right] } & =(n-m) L_{n+m}+\frac{1}{12} c\left(n^{3}-n\right) \delta_{n+m} \\
{\left[L_{n}, J_{m}^{a}\right] } & =-m J_{n+m}^{a} \\
{\left[J_{n}^{a}, J_{m}^{b}\right] } & =f_{b c}^{a} J_{n+m}^{c}+\frac{1}{2} k n \delta^{a b} \delta_{n+m, 0} .
\end{aligned}
$$

This is again a way to write the semi-direct product of the Virasoro (Eq. (2.41a)) and Kač-Moody algebras (Eq. (2.41c)).

\section{The WZW model}

As we emphasized above the currents $J$ and $\bar{J}$ are the fundamental building blocks for the construction of WZW models. Their role is even more apparent when we study the symmetries of the theory, which takes us directly to make contact with the conformal field theory description. Hence the importance of these models in giving an explicit realization of the CFT outlined above (among the classical references see e.g. [KZ84]).

The key remark is that the action in Eq. (2.19) is invariant under the transformation

$$
g(\zeta) \mapsto \Omega(z) g(\zeta) \bar{\Omega}^{-1}(\bar{z})
$$

where $\Omega(z)$ and $\bar{\Omega}(\bar{z})$ are $G$-valued matrices analytically depending on $z$ and $\bar{z}$. This gives rise to an infinite set of conserved currents which are precisely those we defined above in Eq. (2.11) and Eq. (2.33). Locally this translates into the fact that for an infinitesimal transformation

$$
\Omega(z)=\mathbb{1}+\omega(z)=\mathbb{1}+\omega^{a}(z) t^{a}
$$

the currents change as in Eq. (2.34c)

$$
\delta_{\omega} J=[\omega(z), J(z)]+\frac{1}{2} k \omega^{\prime}(z)
$$


which is to say that $J$ and $\bar{J}$ represent an affine Lie algebra with central charge $k$.

The next step consist in identifying the stress-energy tensor. In the classical theory this is a bilinear of the currents, so it is natural to choose the so-called Sugawara construction [Sug68, Som68, KZ84]

$$
T(z)=\frac{1}{2\left(k+g^{*}\right)} \sum_{a} J^{a}(z) J^{a}(z)
$$

where the constant factor is fixed by asking a current to be a weight one operator ${ }^{2}$. Note in particular the fact that the level $k$ is shifted to $k+g^{*}$ which is precisely the same correction we found summing the instanton corrections in the previous section. A simple calculation of the OPE of $T$ with itself gives the value for the central charge of the model

$$
c=\frac{k \operatorname{dim} G}{k+g^{*}}
$$

Using the definition of primary field (and in particular the fact that they are annihilated by $J_{-1}^{a} t_{l}^{a}-L_{-1}$ ) one can easily show that each primary is degenerate and has weight

$$
\Delta=\frac{c_{l}}{g^{*}+k}
$$

where $c_{l}=t_{l}^{a} t_{l}^{a}$ is the quadratic Casimir and in particular coincides with the dual Coxeter number $c_{l}=g^{*}$ if the field transforms in the adjoint representation.

\section{Partition function}

Affine characters As one would expect, a modular invariant partition function for a WZW group can be build entirely out of group theoretical objects. In particular the building blocks are given by the affine characters, ie the generating functions of the weight multiplicities for a given irrep $\Lambda$ that take into account the conformal dimension of the highest weight of the representation:

$$
\chi^{\Lambda}(\tau, v, u)=e^{-2 \imath \pi k u} \operatorname{tr}_{r e p(\Lambda)}\left[q^{L_{0}-c / 24} e^{2 \imath \pi \kappa(v, \mathcal{J})}\right]=e^{2 \imath \pi \tau \frac{\kappa(\Lambda, \Lambda+2 \rho)}{2\left(k+\mathfrak{g}^{*}\right)}-c / 24} c_{\Lambda}(\tau, v, u)
$$

where

- $c h_{\Lambda}(\tau, v, u)$ is the usual character for the affine Lie algebra $\hat{\mathfrak{g}}$ :

$$
c h_{\Lambda}(\tau, v, u)=e^{-2 \imath \pi k u} \sum_{\hat{\lambda} \in \operatorname{Rep}(\Lambda)} \operatorname{dim} V_{\hat{\lambda}} \exp \left\{2 \imath \pi \tau n+\sum_{i} v_{i} \kappa\left(e_{i}, \hat{\lambda}\right)\right\} ;
$$

\footnotetext{
${ }^{2}$ It follows easily that a Kač-Moody primary is a Virasoro primary but not the other way round. Pictorially Eq. (2.37b) is the "square root" of Eq. (2.41a)
} 
- $V_{\hat{\lambda}}$ is the multiplicity of the weight $\hat{\lambda}=(\lambda, k, n)$;

- $\left\langle e_{i}\right\rangle$ is a basis in the root space;

- $\rho=\sum_{\alpha>0} \alpha / 2$ is the Weyl vector.

An alternative way of writing the same characters is obtained in terms of theta functions. To each weight one can assign a theta-function defined as:

$$
\Theta_{\hat{\lambda}}(\tau, v, u)=e^{-2 \imath \pi k u} \sum_{\gamma \in \mathbf{M}_{\mathrm{L}}+\frac{\lambda}{k}} e^{\imath \pi \tau k \kappa(\gamma, \gamma)} e^{2 \imath \pi k \kappa(v, \gamma)}
$$

where $\mathbf{M}_{\mathrm{L}}$ is the long root lattice. Then, using the Weyl-Kač formula the characters are written as:

$$
\chi^{\Lambda}(\tau, v, u)=\frac{\sum_{w \in \mathbf{W}} \epsilon(w) \Theta_{w(\hat{\Lambda}+\hat{\rho})}(\tau, v, u)}{\sum_{w \in \mathbf{W}} \epsilon(w) \Theta_{w(\hat{\rho})}(\tau, v, u)},
$$

$\mathbf{W}$ being the Weyl group of the algebra and $\epsilon(w)$ the parity of the element $w$.

Knowing that the affine Lie algebra is the largest chiral symmetry of the theory it is not surprising that the partition function can be written as

$$
Z=\sum_{\Lambda, \bar{\Lambda}} M^{\Lambda \bar{\Lambda}} \chi^{\Lambda}(\tau, 0,0) \bar{\chi}^{\bar{\Lambda}}(\bar{\tau}, 0,0)
$$

where the sum runs over left and right representations of $\mathfrak{g}$ with highest weight $\Lambda$ and $\bar{\Lambda}$ and $M^{\Lambda \bar{\Lambda}}$ is the mass matrix which is chosen so to respect the modular invariance of $Z$.

A generalization that we will use in the following is obtained for heterotic strings where the $N=(1,0)$ local supersymmetry requires a super-affine Lie algebra for the left sector. The latter can anyway be decoupled in terms of the bosonic characters above and free fermion characters as to give:

$$
Z\left[\begin{array}{l}
a \\
b
\end{array}\right]=\sum_{\Lambda, \bar{\Lambda}} M^{\Lambda \bar{\Lambda}} \chi^{\Lambda}(\tau)\left(\frac{\vartheta\left[\begin{array}{l}
a \\
b
\end{array}\right](\tau)}{\eta(\tau)}\right)^{\operatorname{dim}(\mathfrak{g}) / 2} \bar{\chi}^{\bar{\Lambda}}
$$

where $(a, b)$ are the spin structures of the world-sheet fermions. The characters of the affine algebras can be decomposed according to the generalized parafermionic decomposition, by factorizing the abelian subalgebra of the Cartan torus. For example, we can decompose the left supersymmetric $\mathfrak{g}_{k}$ characters in terms of characters of the supersymmetric coset, given by the following branching relation (see [KS89b]):

$$
\chi^{\Lambda}\left(\frac{\vartheta\left[\begin{array}{l}
a \\
b
\end{array}\right]}{\eta}\right)^{\operatorname{dim}(\mathfrak{j}) / 2}=\sum_{\lambda \bmod \left(k+g^{*}\right) \mathbf{M}_{\mathrm{L}}} \mathcal{P}_{\lambda}^{\Lambda}\left[\begin{array}{l}
a \\
b
\end{array}\right] \frac{\Theta_{\lambda, k+g^{*}}}{\eta^{\operatorname{dim}(\mathfrak{k})}}
$$

in terms of the theta-functions associated to $\mathfrak{g}_{k}$. 


\subsection{The target space point of view}

Supergravity appears as a low energy description of string theory, obtained when asking for the Weyl invariance of the $\sigma$-model Lagrangian. This amounts, at first order in $\alpha^{\prime}$, to the following equations of motion for the metric $g$, the Kalb-Ramond field $B$ and the dilaton $\Phi$ [CMPF85, Tse95]:

$$
\left\{\begin{array}{l}
\beta_{\Phi}=-\frac{1}{2} \nabla^{\mu} \partial_{\mu} \Phi+\partial_{\mu} \Phi \partial^{\mu} \Phi-\frac{1}{24} H_{\mu \rho \sigma} H^{\mu \rho \sigma}, \\
\beta_{g}=R_{\mu \nu}-\frac{1}{4} H_{\mu \rho \sigma} H_{v}{ }^{\rho \sigma}+2 \nabla_{\mu} \nabla_{\nu} \Phi, \\
\beta_{B}=\nabla_{\mu} H_{\nu \rho}^{\mu}+2 \nabla_{\mu} \Phi H^{\mu}{ }_{\nu \rho} .
\end{array}\right.
$$

Being a one-loop calculation, the corresponding results should always be checked against higher order corrections in $\alpha^{\prime}$. On the other hand, as we have already stressed many times above, WZW models (just like the asymmetric deformations we study in this work) only receive corrections in terms of the level of the algebra (or, in this language, on the overall volume of the manifold). This implies that the target space description at one loop in $\alpha^{\prime}$ is automatically correct at all orders. From this point of view, Wess-Zumino-Witten models describe the motion of a string on a group manifold geometry. The background fields are completed by a NS-NS three form $H=\mathrm{d} B$ (Kalb-Ramond field) and a constant dilaton $\Phi=\Phi_{0}$.

The target space analysis is greatly simplified by the fact that the geometric quantities are all naturally expressed in terms of group theoretical objects. Let us consider for concreteness the case of a compact group $G$, whose Lie algebra is generated by $\left\langle t^{\alpha}\right\rangle$ and has structure constants $f_{\beta \gamma}^{\alpha}$. The metric for the group manifold can be chosen as the Killing metric (the choice is unique up to a constant in this case) and it is then natural to use the Maurer-Cartan one-forms as vielbeins. In our conventions, then:

$$
g_{\mu \nu}=-\frac{1}{2 g^{*}} f_{\beta \gamma}^{\alpha} f_{\delta \alpha}^{\gamma} J_{\mu}^{\beta} J_{\nu}^{\delta}=\delta_{\beta \gamma} J_{\mu}^{\beta} J_{\nu}^{\delta},
$$

where $g^{*}$ is the dual Coxeter number and

$$
J^{\alpha}{ }_{\mu}=\left\langle t^{\alpha} g^{-1} \partial_{\mu} g\right\rangle \text {. }
$$

In this basis the NS-NS 3-form field is written as

$$
H_{[3]}=\frac{1}{3 !} f_{\alpha \beta \gamma} J^{\alpha} \wedge J^{\beta} \wedge J^{\gamma} .
$$

Geometry of group manifolds
The connection one-forms $\omega_{\beta}^{\alpha}$ can be obtained by asking for the torsion two-form to vanish:

$$
\mathrm{d} J^{\alpha}+\omega_{\beta}^{\alpha} \wedge J^{\beta}=T^{\alpha}=0,
$$

and out of them one defines the curvature two form $R_{\beta}^{\alpha}$ as:

$$
R_{\beta}^{\alpha}=\mathrm{d} \omega_{\beta}^{\alpha}+\omega_{\gamma}^{\alpha} \wedge \omega_{\beta}^{\gamma} \text {. }
$$


which in turn is given in terms of the Riemann tensor as:

$$
R_{\beta}^{\alpha}=\frac{1}{2} R_{\beta \gamma \delta}^{\alpha} J^{\gamma} \wedge J^{\delta} .
$$

In a Lie algebra with structure constants $f_{\beta \gamma}^{\alpha}$ the variation of the currents is given by the Cartan structure equation:

$$
\mathrm{d} J^{\alpha}=-\frac{1}{2} f_{\beta \gamma}^{\alpha} J^{\beta} \wedge J^{\gamma}
$$

whence we can directly read the connection one-forms:

$$
\omega_{\beta}^{\alpha}=-\frac{1}{2} f_{\beta \gamma}^{\alpha} J^{\gamma} .
$$

It is then immediate to write:

$$
\begin{aligned}
\mathrm{d} \omega_{\beta}^{\alpha} & =\frac{1}{4} f_{\beta \gamma}^{\alpha} f_{\delta \epsilon}^{\gamma} J^{\delta} \wedge J^{\epsilon} \\
\omega_{\gamma}^{\alpha} \wedge \omega_{\beta}^{\gamma} & =\frac{1}{4} f_{\gamma \delta}^{\alpha} f_{\beta \epsilon}^{\gamma} J^{\delta} \wedge J^{\epsilon}=\frac{1}{8}\left(f_{\gamma \delta}^{\alpha} f_{\beta \epsilon}^{\gamma}-f_{\gamma \epsilon}^{\alpha} f_{\beta \delta}^{\gamma}\right) J^{\delta} \wedge J^{\epsilon}= \\
& =-\frac{1}{8} f_{\beta \gamma}^{\alpha} f_{\delta \epsilon}^{\gamma} J^{\delta} \wedge J^{\epsilon}
\end{aligned}
$$

where we have antisimmetrized the product of the structure constants and then used a Jacobi identity. The Riemann tensor, the Ricci tensor and the scalar curvature are then given respectively by:

$$
\begin{aligned}
R_{\beta \gamma \delta}^{\alpha} & =\frac{1}{4} f_{\beta \kappa}^{\alpha} f_{\gamma \delta}^{\mathcal{K}}, \\
\text { Ric }_{\beta \delta} & =\frac{1}{4} f_{\beta \kappa}^{\alpha} f_{\alpha \delta}^{\mathcal{K}}=\frac{g^{*}}{2} g_{\beta \delta}, \\
R & =\frac{g^{*}}{2} \operatorname{dim} G .
\end{aligned}
$$

We are now in a position to show that the metric and $H$ field satisfy the (first order in $\alpha^{\prime}$ ) equations of motion in Eq. (2.55). Of course this result is

Equations of motion much less powerful than what we obtained in Sec. 2.1 but it is nevertheless an interesting example of how these geometrical calculations are greatly simplified in terms of the underlying algebraic structure. For a system without dilaton the equations reduce to:

$$
\begin{aligned}
& \beta_{\alpha \beta}^{G}=R_{\alpha \beta}-\frac{1}{4} H_{\alpha \delta \gamma} H_{\beta}^{\delta \gamma}=0, \\
& \beta_{\alpha \beta}^{B}=\left(\nabla^{\gamma} H\right)_{\gamma \alpha \beta}=0 .
\end{aligned}
$$

The first one is trivially satisfied by using the field in Eq. (2.58); for the second one we just need to remark that in components the Levi-Civita connection is:

$$
\Gamma_{\beta \gamma}^{\alpha}=\frac{1}{2} f_{\beta \gamma}^{\alpha}
$$


and remember that the covariant derivative of a three-form is

$$
\left(\nabla_{\alpha} H\right)_{\beta \gamma \delta}=\partial_{\alpha} H_{\beta \gamma \delta}-\Gamma_{\alpha \beta}^{\kappa} H_{\kappa \gamma \delta}-\Gamma_{\alpha \gamma}^{\kappa} H_{\beta \kappa \delta}-\Gamma_{\alpha \delta}^{\kappa} H_{\beta \gamma \kappa} .
$$

In Sec. 6.1 we will see from a slightly different perspective how the normalization for the Kalb-Ramond field $H$ can be fixed in terms of renormalizationgroup flow. 


\section{CHAPTER 3}

\section{Deformations}

In this rather technical chapter we describe marginal deformations of WessZumino-Witten models. The main purpose for these constructions is to reduce the symmetry of the system while keeping the integrability properties intact, trying to preserve as many nice geometric properties as possible.

Mr. Jabez Wilson laughed heavily. "Well, I never!" said he. "I thought at first that you had done something clever, but I see that there was nothing in it, after all." "I begin to think, Watson," said Holmes, "that I make a mistake in explaining. Omne ignotum pro magnifico, you know, and my poor little reputation, such as it is, will suffer shipwreck if I am so candid."

The Red Headed League ARTHUR CONAN DOYLE

$\mathrm{T}$

HE POWER of WZW models resides in the symmetries of the theory. They impose strong constraints which allow quantum integrability as well as a faithful description in terms of spacetime fields, whose renormalization properties (at every order in $\alpha^{\prime}$ ) are easily kept under control, as we have seen in the previous chapter.

It is hence interesting to study their moduli spaces, aiming at finding less symmetric (and richer) structures, that will hopefully enjoy analogous integrability and spacetime properties.

This chapter is devoted to introducing the construction of asymmetric deformations and giving the general results in a formalism adapted to group manifold geometry. For this reason the stress is put on the more mathematical aspects. Physical examples and consequences will be illustrated in greater detail in Ch. 4. 


\subsection{Deformed WZW models: various perspectives}

\section{Truly marginal deformations}

Truly marginal In this spirit one can consider marginal deformations of the WZW models obdeformations

tained in terms of $(1,1)$ operators built as bilinears in the currents:

$$
\mathcal{O}(z, \bar{z})=\sum_{i j} c_{i j} J^{i}(z) \bar{J}^{j}(\bar{z})
$$

where $J^{i}(z)$ and $\bar{J}^{j}(\bar{z})$ are respectively left- and right-moving currents. It is known [CS89] that this operator represents a truly marginal deformation, ie it remains marginal at all orders in the deformation parameter, if the parameter matrix $c_{i j}$ satisfies the following constraints:

$$
\begin{aligned}
& c_{i m} c_{j n} f_{k}^{i j}=0, \\
& c_{m i} c_{n j} \tilde{f}_{k}^{i j}=0,
\end{aligned}
$$

where $f$ and $\tilde{f}$ are the structure constants of the algebras generated by $J^{i}$ and $\bar{J}^{i}$. In particular one can remark that if $J^{i}$ and $\bar{J}^{j}$ live on a torus then the two equations are automatically satisfied for any value of $c_{m n}$ and hence we get as moduli space, a rank $(c)$-dimensional hyperplane of exact models ${ }^{1}$. The proof of this assertion proceeds as follows: we want to show that $\mathcal{O}$ keeps its conformal dimensions when a term $\mathrm{HO}(z, \bar{z})$ is added to the Lagrangian, $\mathrm{H}$ being a coupling constant. The two-point function for $\mathcal{O}(z, \bar{z})$ in the interacting theory with Lagrangian $L+\mathrm{HO}$ can be expanded in powers of $\mathrm{H}$ as follows:

$$
\begin{aligned}
& \langle\mathcal{O}(z, \bar{z}) \mathcal{O}(w, \bar{w})\rangle_{\mathrm{H}}= \\
& =\frac{\sum_{n=0}^{\infty}(-\mathrm{H})^{n} / n ! \int \mathrm{d}^{2} z_{1} \ldots \mathrm{d}^{2} z_{n}\left\langle\mathcal{O}(z, \bar{z}) \mathcal{O}(w, \bar{w}) \mathcal{O}\left(z_{1}, \bar{z}_{1}\right) \ldots \mathcal{O}\left(z_{n}, \bar{z}_{n}\right)\right\rangle}{\sum_{n=0}^{\infty}(-\mathrm{H})^{n} / n ! \int \mathrm{d}^{2} z_{1} \ldots \mathrm{d}^{2} z_{n}\left\langle\mathcal{O}\left(z_{1}, \bar{z}_{1}\right) \ldots \mathcal{O}\left(z_{n}, \bar{z}_{n}\right)\right\rangle},
\end{aligned}
$$

so, in particular, the $\mathrm{H}^{2}$-order term is:

$$
\begin{aligned}
& \langle\mathcal{O}(z, \bar{z}) \mathcal{O}(w, \bar{w})\rangle_{g}= \\
& =\frac{\mathrm{H}^{2}}{2} \int \mathrm{d}^{2} z_{1} \mathrm{~d}^{2} z_{2}\left\langle\mathcal{O}(z, \bar{z}) \mathcal{O}(w, \bar{w}) \mathcal{O}\left(z_{1}, \bar{z}_{1}\right) \mathcal{O}\left(z_{2}, \bar{z}_{2}\right)\right\rangle+ \\
& \quad-\frac{\mathrm{H}^{2}}{2}\langle\mathcal{O}(z, \bar{z}) \mathcal{O}(w, \bar{w})\rangle \int \mathrm{d}^{2} z_{1} \mathrm{~d}^{2} z_{2}\left\langle\mathcal{O}\left(z_{1}, \bar{z}_{1}\right) \mathcal{O}\left(z_{2}, \bar{z}_{2}\right)\right\rangle .
\end{aligned}
$$

\footnotetext{
${ }^{1}$ Although for special values of the level $k$ the theory contains other operators with the right conformal weights, it is believed that only current-current operators give rise to truly marginal deformations, i.e. operators that remain marginal for finite values of the deformation parameter.
} 
Only the first term can contain logarithmic divergences that can alter the scale dependence of $\mathcal{O}(z, \bar{z})$, so let us study it more closely, by expanding $\mathcal{O}(z, \bar{z})$ in terms of currents:

$$
\begin{aligned}
& \frac{\mathrm{H}^{2}}{2} \int \mathrm{d}^{2} z_{1} \mathrm{~d}^{2} z_{2}\left\langle\mathcal{O}(z, \bar{z}) \mathcal{O}(w, \bar{w}) \mathcal{O}\left(z_{1}, \bar{z}_{1}\right) \mathcal{O}\left(z_{2}, \bar{z}_{2}\right)\right\rangle= \\
& =\frac{\mathrm{H}^{2}}{2} \int \mathrm{d}^{2} z_{1} \mathrm{~d}^{2} z_{2} \sum_{\text {ghij lmno }} \sum_{g h} c_{h m} c_{i n} c_{j o}\left\langle J_{g}(z) J_{h}(w) J_{i}\left(z_{1}\right) J_{j}\left(z_{2}\right)\right\rangle \\
& \left\langle\bar{J}_{l}(\bar{z}) \bar{J}_{m}(\bar{w}) \bar{J}_{n}\left(\bar{z}_{1}\right) \bar{J}_{o}\left(\bar{z}_{2}\right)\right\rangle .
\end{aligned}
$$

Rewriting the four-point functions for the currents in terms of their algebras

$$
\begin{aligned}
& J_{i}(z) J_{j}(w)=\frac{K_{i j}}{(z-w)^{2}}+\frac{\imath f_{i j}^{k} J_{k}(w)}{z-w}, \\
& \bar{J}_{i}(z) \bar{J}_{j}(w)=\frac{\tilde{K}_{i j}}{(z-w)^{2}}+\frac{\imath \tilde{f}_{i j}^{k} \bar{J}_{k}(w)}{z-w},
\end{aligned}
$$

one can evaluate the integrals passing to momentum space and introducing some ultraviolet cut-offs $\Lambda_{1}, \Lambda_{2}, \Lambda$. In particular, the terms which are interesting from our point of view are those diverging as $|z-w|^{-4}$ and they are:

$$
\frac{8 \pi^{2} \mathrm{H}^{2} \log \Lambda_{1} \log \Lambda_{2}}{|z-w|^{4}} \sum_{\text {ghij }} \sum_{\text {lmno }} \sum_{k p} c_{g l} c_{h m} c_{i n} c_{j o} K_{k k} \tilde{K}_{p p} f_{h k}^{g} f_{j k}^{i} \tilde{f}_{m p}^{l} \tilde{f}_{o p}^{n}
$$

and

$\frac{6 \pi^{2} \mathrm{H}^{2} \log \Lambda}{|z-w|^{4}} \sum_{g h i j} \sum_{k l m} c_{g l} c_{h l} c_{i m} c_{j m} K_{k k} \tilde{K}_{l l} \tilde{K}_{m m} f^{g}{ }_{i k} f_{j k}^{h}+c_{l g} c_{l h} c_{m i} c_{m j} \tilde{K}_{k k} K_{l l} K_{m m} \tilde{f}_{i k}^{g} \tilde{f}_{j k}^{h}$.

Using the fact that the matrices $K_{i j}$ and $\tilde{K}_{i j}$ are positive-definite it is simple to see that they both vanish if and only if Eq. (3.2) are satisfied (the condition is only sufficient for general semi-simple groups).

Actually there's another piece of information that we learn out of this construction: the OPE coefficients among the currents used for the deformation do not change with the deformation. As we will see in the next section, this implies that the the effect of the deformation is completely captured by a transformation in the charge lattice of the theory.

\section{Algebraic structure of current-current deformations}

The result of the previous section can be recast in more abstract terms: consider a conformal field theory whose holomorphic and anti-holomorphic Kac Moody algebras correspond to Lie algebras $\mathfrak{g}$ and $\overline{\mathfrak{g}}$, which respectively admit the abelian subalgebras $\mathfrak{h}$ and $\overline{\mathfrak{h}}$. Then each pair $\mathfrak{u}(1)^{d} \subseteq \mathfrak{h}, \mathfrak{u}(1)^{\bar{d}} \subseteq \overline{\mathfrak{h}}$ gives rise to a new family of conformal field theory containing those algebras (the ones 
defined in Eqs. (3.6)). It is safe to assume (at least in the compact case) that the CFT remains unitary and that its Hilbert space still decomposes into tensor products of irreducible highest weight representations of $\mathfrak{h} \times \overline{\mathfrak{h}}$ (from now on $\operatorname{dim} \mathfrak{h}=d$ and $\operatorname{dim} \overline{\mathfrak{h}}=\bar{d}$ )

$$
\mathcal{H}=\sum_{Q, \bar{Q}} \mathcal{H}_{Q, \bar{Q}} \mathcal{V}_{Q} \otimes \mathcal{V}_{\bar{Q}}
$$

where we used the fact that those representations are completely characterized by their charges $(Q, \bar{Q}) \in\left(\mathfrak{h}^{*}, \overline{\mathfrak{h}}^{*}\right)$ and the corresponding conformal weights are given by $h=1 / 2 \kappa(Q, Q)$ and $\bar{h}=1 / 2 \overline{\mathcal{K}}(\bar{Q}, \bar{Q})$, where $\kappa$ and $\bar{\kappa}$ are the Killing forms respectively on $\mathfrak{g}$ and $\overline{\mathfrak{g}}$ restricted on $\mathfrak{h}$ and $\overline{\mathfrak{h}}$. This set of charges nat-

Boost on the charge lattice urally forms a lattice $\Lambda$ when equipped with the pairing $\langle\rangle=,\kappa-\bar{\kappa}$. Using in example deformation theory as in [FR03] one can see that the effect of the deformation is completely captured by an $O(d, \bar{d})$ pseudo-orthogonal transformation of this charge lattice $\Lambda \subset \mathfrak{h}^{*} \times \overline{\mathfrak{h}}^{*}$, ie can be described in terms of the identity component of the group $O(d, \bar{d})$. Moreover, since the charges only characterise the $\mathfrak{h} \times \overline{\mathfrak{h}}$ modules up to automorphisms of the algebras, $O(d) \times O(\bar{d})$ transformations don't change the CFT. Hence the deformation space is given by:

$$
D_{\mathfrak{h}, \overline{\mathfrak{h}}} \sim O(d, \bar{d}) /(O(d) \times O(\bar{d})) .
$$

The moduli space is obtained out of $D_{\mathfrak{h}, \overline{\mathfrak{h}}}$ after the identification of the points giving equivalent $\mathrm{CFTs}^{2}$.

In the case of WZW models on compact groups, all maximal abelian subgroups are pairwise conjugated by inner automorphisms. This implies that the complete deformation space is $D=O(d, d) /(O(d) \times O(d))$ where $d$ is the rank of the group. The story is different for non-semi-simple algebras, whose moduli space is larger, since we get different $O(d, \bar{d}) /(O(d) \times O(\bar{d}))$ deformation spaces for each (inequivalent) choice of the abelian subalgebras $\mathfrak{h} \subset \mathfrak{g}$ and $\overline{\mathfrak{h}} \subset \overline{\mathfrak{g}}$. We'll see an example of this in the next chapter where deforming a $S L(2, \mathbb{R})$ WZW model (Sec. 4.2 and Sec. 4.4) will give rise to a much richer structure than in the $S U(2)$ case (Sec. 4.1).

Parafermion decomposition

Truly marginal deformations of WZW model single out abelian subalgebras of the model. It is then natural that an important tool in describing these current-current deformations comes from the so-called parafermion decomposition. The highest-weight representation for a $\hat{\mathfrak{g}}_{k}$ graded algebra can be decomposed into highest-weight modules of a Cartan subalgebra $\hat{\mathfrak{h}} \subset \hat{\mathfrak{g}}_{k}$ as follows [GQ87, Gep87]:

$$
\mathcal{V}_{\hat{\lambda}} \simeq \bigoplus_{\mu \in \Gamma_{k}} \mathcal{V}_{\hat{\lambda}, \mu} \otimes \bigoplus_{\delta \in Q_{l}(\mathfrak{g})} \mathcal{V}_{\mu+k \delta},
$$

where $\hat{\lambda}$ is an integrable weight of $\hat{\mathfrak{g}}_{k}, \mathcal{V}_{\hat{\lambda}, \mu}$ is the highest-weight module for the generalized $\hat{\mathfrak{g}}_{k} / \hat{\mathfrak{h}}$ parafermion, $Q_{l}(\mathfrak{g})$ is the long-root lattice and $\Gamma_{k}=P(\mathfrak{g}) / Q_{l}(\mathfrak{g})$

\footnotetext{
${ }^{2}$ Although we will concentrate on WZW models it is worth to emphasize that this construction is more general.
} 
with $P(\mathfrak{g})$ the weight lattice. As a consequence, the WZW model based on $\hat{\mathfrak{g}}_{k}$ can be represented as an orbifold model:

$$
\hat{\mathfrak{g}}_{k} \simeq\left(\hat{\mathfrak{g}}_{k} / \hat{\mathfrak{h}} \otimes t_{\Lambda_{k}}\right) / \Gamma_{k}
$$

where $t_{\Lambda_{k}}$ is a toroidal CFT with charge lattice, included in the $\hat{\mathfrak{g}}_{k}$ one, defined as $\Lambda_{k}=\left\{(\mu, \bar{\mu}) \in P(\hat{\mathfrak{g}}) \times P(\hat{\mathfrak{g}}) \mid \mu-\bar{\mu}=k Q_{l}(\hat{\mathfrak{g}})\right\}$. In our case the advantage given by using this representation relies on the fact that $\Gamma_{k}$ acts trivially on the coset and toroidal model algebras; then, if we identify $\hat{\mathfrak{h}}$ and $\overline{\hat{h}}$ with the graded algebras of $t_{\Lambda_{k}}$, the deformation only acts on the toroidal lattice and the deformed model can again be represented as an orbifold:

$$
\hat{\mathfrak{g}}_{k}(\mathcal{O}) \simeq\left(\hat{\mathfrak{g}}_{k} / \hat{\mathfrak{h}} \otimes t_{\mathcal{O} \Lambda_{k}}\right) / \Gamma_{k}
$$

where $\mathcal{O}$ is an operator in the moduli space. In other words this representation is specially useful because it allows to easily single out the sector of the theory that is affected by the deformation. As we'll see in the next section this simplifies the task of writing the corresponding Lagrangian.

In the following we will separate this kind of deformations into two categories: those who give rise to symmetric deformations, i.e. the ones where $c_{i j}=\delta_{i j}$ and $J^{i}(z)$ and $\tilde{J}^{j}(\bar{z})$ represent the same current in the two chiral sectors of the theory and the asymmetric ones where the currents are different and in general correspond to different subalgebras. This distinction is somehow arbitrary, since both symmetric and asymmetric deformations act as $O(d, \bar{d})$ rotations on the background fields. It is nonetheless interesting to single out the asymmetric case. In the special situation when one of the two currents belongs to an internal $U(1)$ (coming from the gauge sector in the heterotic or simply from any $U(1)$ subalgebra in the type II), it is in fact particularly simple to study the effect of the deformation, even from the spacetime field point of view; there in fact, the expressions for the background fields are exact (at all order in $\alpha^{\prime}$ and for every value of the level $k$ ) as we will show in Sec. 3.4.

\section{Background fields and symmetric deformations}

Before moving to the asymmetric deformations we're interested in, let us consider briefly symmetric deformations (also called gravitational) which are those that have received by far the most attention in literature [HS93, GK94, F9̈4, FR03, DOPS05]. Specialising Eq. (3.1) to the case of one only current we can write the small deformation Lagrangian as:

$$
S=S_{\mathrm{WZW}}+\delta \kappa^{2} \int \mathrm{d}^{2} z J(z) \bar{J}(\bar{z})
$$

This infinitesimal deformation has to be integrated in order to give a Lagrangian interpretation to the CFT described in the previous section. Different approaches are possible, exploiting the different possible representations described above.

Symmetric and asymmetric deformations 
- A possible way consists in implementing an $O(d, d)$ rotation on the background fields [HS93]. More precisely, one has to identify a coordinate system in which the background fields are independent of $d$ space dimensions and metric and $B$ field are written in a block diagonal form. In this way the following matrix is defined:

$$
M=\left(\begin{array}{c|c}
\hat{g}^{-1} & -\hat{g}^{-1} \hat{B} \\
\hline \hat{B} \hat{g}^{-1} & \hat{g}-\hat{B} \hat{g}^{-1} \hat{B}
\end{array}\right),
$$

where $\hat{g}$ and $\hat{B}$ are the pull-backs of the metric and Kalb-Ramond field on the $p$ selected directions. Then the action of the $O(d, d)$ group on these fields and dilaton is given by:

$$
\begin{aligned}
& M \rightarrow M^{\prime}=\Omega M \Omega^{t}, \\
& \Phi \rightarrow \Phi^{\prime}=\Phi+\frac{1}{2} \log \left(\frac{\operatorname{det} \hat{g}}{\operatorname{det} \hat{g}^{\prime}}\right),
\end{aligned}
$$

where $\hat{g}^{\prime}$ is the metric after the transformation (3.15a) and $\Omega \in O(d, d)$. It must be emphasized that this transformation rules are valid at the lowest order in $\alpha^{\prime}$ (but for finite values of the deformation parameters). So, although the model is exact, as we learn from the CFT side, the field expressions that we find only are true at leading order in $\alpha^{\prime}$.

- An alternative approach uses the parafermion representation Eq. (3.12) (see e.g. [FR03]). In practice this amounts to writing an action as the sum of the $G / H$ parafermion and a deformed $H$ part and finding the appropriate T-duality transformation (realizing the orbifold) such that for zero deformation the WZW on G is recovered, in accordance with Eq. (3.11).

- Finally, another point of view (inspired by the parafermionic representation), consists in identifying the deformed model with a $(G \times H) / H$ coset model, in which the embedding of the dividing group has a component in both factors [GK94]. The gauging of the component in G gives the parafermionic sector, the gauging of the component in $H$ gives the deformed toroidal sector and the coupling term (originating from the quadratic structure in the fields introduced for the gauging) corresponds to the orbifold projection ${ }^{3}$.

\subsection{Background fields for the asymmetric deformation}

Let us now consider the less-known case of asymmetric deformations, in which the two sets of currents $J_{i}$ and $\bar{J}_{j}$ come from distinct sectors of the theory. The

\footnotetext{
${ }^{3}$ An instanton-correction-aware technique that should overcome the first order in $\alpha^{\prime}$ limitation for gauged models has been proposed in [Tse94]. In principle this can be used to get an all-order exact background when we write the deformation as a gauged model. We will not expand further in this direction, that could nevertheless be useful to address issues such as the stability of the black string (see Sec. 4.4).
} 
archetype of such construction is what we get considering an heterotic superWZW model on a group $G$ at level $k$ and adding an exactly marginal operator built from the total Cartan currents of $\mathfrak{g}$ (so that it preserves the local $N=(1,0)$ superconformal symmetry of the theory):

$$
S=S_{\mathrm{WZW}}+\frac{\sqrt{k k_{g}}}{2 \pi} \int \mathrm{d}^{2} z \sum_{a} \mathrm{H}_{a}\left(J^{a}(z)-\frac{i}{k} f_{\mathrm{MN}}^{a}: \psi^{\mathrm{M}} \psi^{\mathrm{N}}:\right) \bar{J}(\bar{z})
$$

where the set $\left\{\mathrm{H}_{a}\right\}$ are the parameters of the deformation, $J^{a}$ are currents in the maximal torus $T \subset G$ and $\bar{J}(\bar{z})$ is a right moving current of the Cartan subalgebra of the heterotic gauge group at level $k_{g}$. Such a deformation is always truly marginal since the $J_{a}$ currents commute.

It is not completely trivial to read off the deformed background fields that correspond to the deformed action. A possible way is a method involving a Kaluza-Klein reduction as in [HT95]. For simplicity we will consider the

Reading the squashed group fields bosonic string with vanishing dilaton and just one operator in the Cartan subalgebra $k$. After bosonization the right-moving gauge current $\bar{J}$ used for the deformation has now a left-moving partner and can hence be written as $\bar{J}=\imath \bar{\partial} \varphi$, $\varphi(z, \bar{z})$ being interpreted as an internal degree of freedom. The sigma-model action is recast as

$$
S=\frac{1}{2 \pi} \int \mathrm{d}^{2} z\left(G_{\mathrm{MN}}+B_{\mathrm{MN}}\right) \partial x^{\mathrm{M}} \bar{\partial} x^{\mathrm{N}},
$$

where the $x^{\mathrm{M}}, \mathrm{M}=1, \ldots, d+1$ embrace the group coordinates $x^{\mu}, \mu=1, \ldots, d$ and the internal $x^{d+1} \equiv \varphi$ :

$$
x^{\mathrm{M}}=\left(\frac{x^{\mu}}{\varphi}\right) \text {. }
$$

If we split accordingly the background fields, we obtain the following decomposition:

$$
G_{\mathrm{MN}}=\left(\begin{array}{c|c}
G_{\mu \nu} & G_{\varphi \varphi} A_{\mu} \\
\hline G_{\varphi \varphi} A_{\mu} & G_{\varphi \varphi}
\end{array}\right), \quad B_{\mathrm{MN}}=\left(\begin{array}{c|c}
B_{\mu \nu} & B_{\mu \varphi} \\
\hline-B_{\mu \varphi} & 0
\end{array}\right),
$$

and the action becomes:

$$
\begin{array}{r}
S=\frac{1}{2 \pi} \int \mathrm{d} z^{2}\left\{\left(G_{\mu \nu}+B_{\mu \nu}\right) \partial x^{\mu} \bar{\partial} x^{\nu}+\left(G_{\varphi \varphi} A_{\mu}+B_{\mu \varphi}\right) \partial x^{\mu} \bar{\partial} \varphi\right. \\
\left.+\left(G_{\varphi \varphi} A_{\mu}-B_{\mu \varphi}\right) \partial \varphi \bar{\partial} x^{\mu}+G_{\varphi \varphi} \partial \varphi \bar{\partial} \varphi\right\}
\end{array}
$$

We would like to put the previous expression in such a form that spacetime gauge invariance,

$$
\begin{aligned}
A_{\mu} & \rightarrow A_{\mu}+\partial_{\mu} \lambda \\
B_{\mu 4} & \rightarrow B_{\mu 4}+\partial_{\mu} \eta
\end{aligned}
$$


is manifest. This is achieved as follows:

$$
\begin{aligned}
& S=\frac{1}{2 \pi} \int \mathrm{d}^{2} z\left\{\left(\hat{G}_{\mu \nu}+B_{\mu \nu}\right)\right. \partial x^{\mu} \bar{\partial} x^{\nu}+B_{\mu \varphi}\left(\partial x^{\mu} \bar{\partial} \varphi-\partial \varphi \bar{\partial} x^{\mu}\right)+ \\
&\left.+G_{\varphi \varphi}\left(\partial \varphi+A_{\mu} \partial x^{\mu}\right)\left(\bar{\partial} \varphi+A_{\mu} \bar{\partial} x^{\mu}\right)\right\},
\end{aligned}
$$

where $\hat{G}_{\mu \nu}$ is the Kaluza-Klein metric

$$
\hat{G}_{\mu \nu}=G_{\mu \nu}-G_{\varphi \varphi} A_{\mu} A_{\nu}
$$

We can then make the following identifications:

$$
\begin{aligned}
\hat{G}_{\mu v} & =k\left(\mathcal{J}_{\mu} \mathcal{J}_{v}-2 \mathrm{H}^{2} \tilde{\mathcal{J}}_{\mu} \tilde{\mathcal{J}}_{\nu}\right) \\
B_{\mu v} & =k \mathcal{J}_{\mu} \wedge \mathcal{J}_{\nu} \\
B_{\mu \varphi} & =G_{\varphi \varphi} A_{\mu}=\mathrm{H} \sqrt{k k_{g}} \tilde{\mathcal{J}}_{\mu,} \\
A_{\mu} & =2 \mathrm{H} \sqrt{\frac{k}{k_{g}}} \tilde{\mathcal{J}}_{\mu}, \\
G_{\varphi \varphi} & =\frac{k_{g}}{2} .
\end{aligned}
$$

where $\tilde{\mathcal{J}}$ is the Maurer-Cartan current chosen for the deformation. Let us now consider separately the background fields we obtained so to give a clear geometric interpretation of the deformation, in particular in correspondence of what we will find to be the maximal value for the deformation parameters $\mathrm{H}_{a}$.

Metric on The metric. According to Eq. (3.25a), in terms of the target space metric, squashed group and decompactification limit the effect of this perturbation amounts to inducing a back-reaction that in the vielbein (current) basis is written as:

$\langle\mathrm{d} g, \mathrm{~d} g\rangle_{\mathrm{H}}=\sum_{M} \mathcal{J}_{M} \otimes \mathcal{J}_{M}-2 \sum_{a} \mathrm{H}_{a}^{2} \mathcal{J}_{a} \otimes \mathcal{J}_{a}=\sum_{\mu} \mathcal{J}_{\mu} \otimes \mathcal{J}_{\mu}+\sum_{a}\left(1-2 \mathrm{H}_{a}^{2}\right) \mathcal{J}_{a} \otimes \mathcal{J}_{a}$

where we have explicitly separated the Cartan generators. From this form of the deformed metric we see that there is a "natural" maximal value $\mathrm{H}_{a}=1 / \sqrt{2}$ where the contribution of the $\mathcal{J}_{a} \otimes \mathcal{J}_{a}$ term changes its sign and the signature of the metric is thus changed. One could naively think that the maximal value $\mathrm{H}_{a}=1 / \sqrt{2}$ can't be attained since the this would correspond to a degenerate manifold of lower dimension; what actually happens is that the deformation selects the the maximal torus that decouples in the $\mathrm{H}_{a}=\mathrm{H} \rightarrow 1 / \sqrt{2}$ limit.

To begin, write the general element $g \in G$ as $g=h t$ where $h \in G / T, t \in T$. Substituting this decomposition in the expression above we find:

$$
\begin{gathered}
\langle\mathrm{d}(h t), \mathrm{d}(h t)\rangle_{\mathrm{H}}=\left\langle(h t)^{-1} \mathrm{~d}(h t)(h t)^{-1} \mathrm{~d}(h t)\right\rangle-\sum_{a} 2 \mathrm{H}_{a}^{2}\left\langle T_{a}(h t)^{-1} \mathrm{~d}(h t)^{-1}\right\rangle^{2}= \\
=\left\langle h^{-1} \mathrm{~d} h h^{-1} \mathrm{~d} h\right\rangle+2\left\langle\mathrm{~d} t t^{-1} h^{-1} \mathrm{~d} h\right\rangle+\left\langle t^{-1} \mathrm{~d} t t^{-1} \mathrm{~d} t\right\rangle+ \\
\quad-\sum_{a} 2 \mathrm{H}_{a}^{2}\left(\left\langle T_{a} t^{-1} h^{-1} \mathrm{~d} h\right\rangle+\left\langle T_{a} t^{-1} \mathrm{~d} t\right\rangle\right)^{2}
\end{gathered}
$$


let us introduce a coordinate system $\left(\gamma_{\mu}, \psi_{a}\right)$ such as the element in $G / T$ is parametrized as $h=h\left(\gamma_{\mu}\right)$ and $t$ is written explicitly as:

$$
t=\exp \left\{\sum_{a} \psi_{a} T_{a}\right\}=\prod_{a} e^{\psi_{a} T_{a}}
$$

it is easy to see that since all the $T_{a}$ commute $t^{-1} \mathrm{~d} t=\mathrm{d} t t^{-1}=\sum_{a} T_{a} \mathrm{~d} \psi_{a}$. This allows for more simplifications in the above expression that becomes:

$$
\begin{array}{r}
\langle\mathrm{d}(h t), \mathrm{d}(h t)\rangle_{\mathrm{H}}=\left\langle h^{-1} \mathrm{~d} h h^{-1} \mathrm{~d} h\right\rangle+2 \sum_{a}\left\langle T_{a} h^{-1} \mathrm{~d} h\right\rangle \mathrm{d} \psi_{a}+\sum_{a} \mathrm{~d} \psi_{a} \mathrm{~d} \psi_{a}+ \\
-\sum_{a} 2 \mathrm{H}_{a}^{2}\left(\left\langle T_{a} h^{-1} \mathrm{~d} h\right\rangle+\mathrm{d} \psi_{a}\right)^{2}=\left\langle h^{-1} \mathrm{~d} h h^{-1} \mathrm{~d} h\right\rangle-\sum_{a} 2 \mathrm{H}_{a}^{2}\left\langle T_{a} h^{-1} \mathrm{~d} h\right\rangle^{2}+ \\
\quad+2 \sum_{a}\left(1-2 \mathrm{H}_{a}^{2}\right)\left\langle T_{a} h^{-1} \mathrm{~d} h\right\rangle \mathrm{d} \psi_{a}+\sum_{a}\left(1-2 \mathrm{H}_{a}^{2}\right) \mathrm{d} \psi_{a} \mathrm{~d} \psi_{a}
\end{array}
$$

if we reparametrise the $\psi_{a}$ variables as:

$$
\psi_{a}=\frac{\hat{\psi}_{a}}{\sqrt{1-2 \mathrm{H}_{a}}}
$$

we get a new metric $\langle\cdot, \cdot\rangle_{\mathrm{H}}^{\prime}$ where we're free to take the $\mathrm{H}_{a} \rightarrow 1 / \sqrt{2}$ limit:

$$
\begin{aligned}
\langle\mathrm{d}(h t), \mathrm{d}(h t)\rangle_{\mathrm{H}}^{\prime}= & \left\langle h^{-1} \mathrm{~d} h h^{-1} \mathrm{~d} h\right\rangle-\sum_{a} 2 \mathrm{H}_{a}^{2}\left\langle T_{a} h^{-1} \mathrm{~d} h\right\rangle^{2}+ \\
& +2 \sum_{a} \sqrt{1-2 \mathrm{H}_{a}^{2}}\left\langle T_{a} h^{-1} \mathrm{~d} h\right\rangle \mathrm{d} \hat{\psi}_{a}+\sum_{a} \mathrm{~d} \hat{\psi}_{a} \mathrm{~d} \hat{\psi}_{a}
\end{aligned}
$$

and get:

$$
\langle\mathrm{d}(h t), \mathrm{d}(h t)\rangle_{1 / \sqrt{2}}^{\prime}=\left[\left\langle h^{-1} \mathrm{~d} h h^{-1} \mathrm{~d} h\right\rangle-\sum_{a}\left\langle T_{a} h^{-1} \mathrm{~d} h\right\rangle^{2}\right]+\sum_{a} \mathrm{~d} \psi_{a} \mathrm{~d} \psi_{a}
$$

where we can see the sum of the restriction of the Cartan-Killing metric ${ }^{4}$ on $T_{h} G / T$ and the metric on $T_{t} T=T_{t} U(1)^{r}$. In other words the coupling terms between the elements $h \in G / T$ and $t \in T$ vanished and the resulting metric $\langle\cdot, \cdot\rangle_{1 / \sqrt{2}}^{\prime}$ describes the tangent space $T_{h t}$ to the manifold $G / T \times T$.

Other Background fields. The asymmetric deformation generates a non-trivial field strength for the gauge field, that from Eq. (3.25d) is found to be:

$$
F^{a}=2 \sqrt{\frac{k}{k_{g}}} \mathrm{H}_{a} \mathrm{~d} \mathcal{J}^{a}=-\sqrt{\frac{k}{k_{g}}} \mathrm{H}_{a} f^{a}{ }_{\mu \nu} J^{\mu} \wedge J^{v}
$$

\footnotetext{
${ }^{4}$ This always is a left-invariant metric on $G / H$. A symmetric coset doesn't admit any other metric. For a more complete discussion see Sec. 3.3
} 
(no summation implied over $a$ ).

On the other hand, the $B$-field (3.25b) is not changed, but the physical object is now the 3-form $H_{[3]}$ :

$$
H_{[3]}=\mathrm{d} B-G_{\varphi \varphi} A^{a} \wedge \mathrm{d} A^{a}=\frac{1}{3 !} f_{\mathrm{MNP}} \mathcal{J}^{\mathrm{M}} \wedge \mathcal{J}^{\mathrm{N}} \wedge \mathcal{J}^{\mathrm{P}}-\sum_{a} \mathrm{H}_{a}^{2} f_{a \mathrm{NP}} \mathcal{J}^{a} \wedge \mathcal{J}^{\mathrm{N}} \wedge \mathcal{J}^{\mathrm{P}}
$$

where we have used the Maurer-Cartan structure equations. At the point where the fibration trivializes, $\mathrm{H}_{a}=1 / \sqrt{2}$, we are left with:

$$
H_{[3]}=\frac{1}{3 !} f_{\mu v \rho} \mathcal{J}^{\mu} \wedge \mathcal{J}^{v} \wedge \mathcal{J}^{\rho}
$$

So only the components of $H_{[3]}$ "living" in the coset $G / T$ survive the deformation. They are not affected of course by the rescaling of the coordinates on $T$.

A trivial fibration. The whole construction can be reinterpreted in terms of fibration as follows. The maximal torus $T$ is a closed Lie subgroup of the Lie group $G$, hence we can see $G$ as a principal bundle with fiber space $T$ and base space $G / T$ [Nak]

$$
G \stackrel{T}{\rightarrow} G / T
$$

The effect of the deformation consists then in changing the fiber and the limit value $\mathrm{H}_{a}=1 / \sqrt{2}$ marks the point where the fibration becomes trivial and it is interpreted in terms of a gauge field whose strength is given by the canonical connection on $G / T$ [KN69].

\subsection{Geometry of squashed groups}

In order to describe the squashed group manifolds that we obtain via asymmetric deformation we need to generalize the discussion on group manifold geometry presented in Sec. 2.2. Let $\left\{\hat{\theta}^{\alpha}\right\}$ be a set of one-forms on a manifold $\mathcal{M}$ satisfying the commutation relations

$$
\left[\hat{\theta}^{\beta}, \hat{\theta}^{\gamma}\right]=f_{\beta \gamma}^{\alpha} \hat{\theta}^{\alpha}
$$

as it is the case when $\hat{\theta}^{\alpha}$ are the Maurer-Cartan one-forms of Eq. (2.57) and $f_{\beta \gamma}^{\alpha}$ the structure constants for the algebra. We wish to study the geometry of the Riemann manifold $\mathcal{M}$ endowed with the metric

$$
g=g_{\alpha \beta} \hat{\theta}^{\alpha} \otimes \hat{\theta}^{\beta}
$$

In general such a metric will have a symmetry $G \times G^{\prime}$ where $G$ is the group corresponding to the structure constants $f_{\beta \gamma}^{\alpha}$ and $G^{\prime} \subset G$. The maximally 
symmetric case, in which $G^{\prime}=G$ is obtained when $g$ is $G$-invariant, i.e. when it satisfies

$$
f_{\beta \gamma}^{\alpha} g_{\alpha \delta}+f_{\delta \gamma}^{\alpha} g_{\alpha \beta}=0 .
$$

for compact groups this condition is fulfilled by the Killing metric in Eq. (2.56).

The connection one-forms $\omega^{\alpha}{ }_{\beta}$ are uniquely determined by the compatibility condition and the vanishing of the torsion. Respectively:

$$
\begin{aligned}
& \mathrm{d} g_{\alpha \beta}-\omega^{\gamma}{ }_{\alpha} g_{\gamma \beta}-\omega_{\beta}^{\gamma} g_{\gamma \alpha}=0 \\
& \mathrm{~d} \hat{\theta}^{\alpha}+\omega^{\alpha}{ }_{\beta} \wedge \hat{\theta}^{\beta}=T^{\alpha}=0
\end{aligned}
$$

As it is shown in [MHS88], if $g_{\alpha \beta}$ is constant, the solution to the system can be put in the form

$$
\omega_{\beta}^{\alpha}=-D_{\beta \gamma}^{\alpha} \hat{\theta}^{\gamma}
$$

where $D_{\beta \gamma}^{\alpha}=1 / 2 f_{\beta \gamma}^{\alpha}-K_{\beta \gamma}^{\alpha}$ and $K_{\beta \gamma}^{\alpha}$ is a tensor (symmetric in the lower indices) given by:

$$
K_{\beta \gamma}^{\alpha}=\frac{1}{2} g^{\alpha \kappa} f_{\kappa \beta}^{\delta} g_{\gamma \delta}+\frac{1}{2} g^{\alpha \kappa} f_{\kappa \gamma \delta \beta \delta}^{\delta} g_{\beta \delta}
$$

Just as in Sec. 2.2 we define the curvature two-form $R_{j}^{i}$ and the Riemann tensor Curvature tensors which now reads:

$$
R_{\beta \gamma \delta}^{\alpha}=D_{\beta \kappa}^{\alpha} f_{\gamma \delta}^{\kappa}+D_{\kappa \gamma}^{\alpha} D_{\beta \delta}^{\kappa}-D_{\kappa \delta}^{\alpha} D_{\beta \gamma}^{\kappa}
$$

on squashed groups

and the corresponding Ricci tensor:

$$
\operatorname{Ric}_{\beta \delta}=D_{\beta \kappa}^{\alpha} f_{\alpha \delta}^{\kappa}-D_{\kappa \delta}^{\alpha} D_{\beta \alpha}^{\kappa}
$$

In particular for $g_{i j} \propto \delta_{i j}, K=0$ so that we recover the usual Maurer-Cartan structure equation Eq. (2.63) and the expressions in Eqs. (2.65).

Let us now specialize these general relations to the case of the conformal model with metric given in Eq. (3.26). The $\hat{\theta}^{i \prime}$ s are the Maurer-Cartan oneforms for the group $G$ and the metric $g_{\mathrm{AB}}$ is

$$
g_{\mathrm{AB}}= \begin{cases}\delta_{\mu v} & \text { if } \mu, v \in G / H \\ \left(1-\frac{\mathrm{H}^{2}}{2}\right) \delta_{a b} & \text { if } a, b \in H\end{cases}
$$

where $H$ is (a subgroup of) the Cartan torus $H \subset G$. It is quite straightforward to show that the Ricci tensor is given by ${ }^{5}$ :

$$
\operatorname{Ric}_{\mathrm{AB}}= \begin{cases}\frac{1}{2}\left(g^{*}+\mathrm{H}^{2}\right) g_{\mu v} & \text { if } \mu, v \in G / H \\ g^{*} / 2\left(1-\frac{\mathrm{H}^{2}}{2}\right) g_{a b} & \text { if } a, b \in H .\end{cases}
$$

$$
\begin{aligned}
& { }^{5} \text { In the } S U(2) \text { case this would be } \\
& g=\left(\begin{array}{lll}
1 & & \\
& 1 & \\
& & 1-\frac{\mathrm{H}^{2}}{2}
\end{array}\right) \quad \text { Ric }=\left(\begin{array}{lll}
1+\frac{\mathrm{H}^{2}}{2} & & \\
& 1+\frac{\mathrm{H}^{2}}{} & \\
& & \left(1-\frac{\mathrm{H}^{2}}{2}\right)^{2}
\end{array}\right)
\end{aligned}
$$

where we chose $J_{3}$ as Cartan generator. 
whence we can read the (constant) Ricci scalar

$$
R=\frac{g^{*}}{2} \operatorname{dim} G+\frac{\mathrm{H}^{2}}{2}\left(\operatorname{dim} G-\operatorname{rank} G\left(1+\frac{g^{*}}{2}\right)\right)
$$

Geometry on $G / H$ cosets

Kähler structure on $G / H$

Particular attention should be devoted to the limit case $\mathrm{H}=\sqrt{2}$ in which the Cartan torus decouples and we are left with the geometry of the $G / T$ coset. In this case it is useful to explicitly write down the commutation relations, separating the generators of $T$ and $G / T$ :

$$
\begin{array}{ll}
{\left[T_{m}, T_{n}\right]=f^{o}{ }_{m n} T_{o}} & {\left[T_{m}, T_{v}\right]=f^{\omega}{ }_{m v} T_{\omega}} \\
{\left[T_{\mu}, T_{v}\right]} & =f_{\mu \nu}^{o} T_{o}+f_{\mu \nu}^{\omega} T_{\omega}
\end{array}
$$

Of course there are no $f^{\omega}{ }_{m n}$ terms since $T$ is a group. $G / T$ is said to be symmetric if $f^{\omega}{ }_{\mu \nu} \equiv 0$, i.e. if the commutator of any couple of coset elements lives in the dividing subgroup. In this case a classical theorem states that the coset only admits one left-invariant Riemann metric that is obtained as the restriction of the Cartan-Killing metric defined on $G$ (see eg [KN69]). This is not the case when $T$ is the maximal torus (except for the most simple case $G=S U$ (2) where maximal torus and maximal subgroup are the trivial $U(1))$ and the coset manifold accepts different structures. From our point of view this means that even when considering deformations and cosets of compact groups where the Cartan subalgebra is unique (up to inner automorphisms), in general we expect different possible outcomes depending on how the gauging is performed (see in particular the $S U(3)$ case studied in detail in Sec. 4.5).

These homogeneous manifolds enjoy many interesting properties. As we pointed out many times already, the best part of them can be interpreted as consequence of the presence of an underlying structure that allows to recast all the geometric problems in Lie algebraic terms. There's however at least one intrinsically geometric property that it is worth to emphasize since it will have many profound implications in the following. All these spaces can be naturally endowed with complex structures by using positive and negative roots as holomorphic and anti-holomorphic generators. This structure doesn't in general correspond to a unique left-invariant Riemann metric. On the other hand there always exists such a metric that is also Kähler. In fact one can easily show that the $(1,1)$ form defined as:

$$
\omega=\frac{l}{2} \sum_{\alpha>0} c_{\alpha} \mathcal{J}^{\alpha} \wedge \mathcal{J}^{\bar{\alpha}}
$$

is closed if and only if for each subset of roots $\{\alpha, \beta, \gamma\}$ such as $\alpha=\beta+\gamma$, the corresponding real coefficients $c_{\alpha}$ satisfy the condition $c_{\alpha}=c_{\beta}+c_{\gamma}$. Of course this is equivalent to say that the tensor

$$
g=\sum_{\alpha>0} c_{\alpha} \mathcal{J}^{\alpha} \otimes \mathcal{J}^{\bar{\alpha}}
$$

is a Kähler metric on $G / T$ [BH58, Per87]. 


\subsection{A no-renormalization theorem}

As we've said many times, WZW models are exact solutions keeping their geometrical description at all orders in $\alpha^{\prime}$, the only effect of renormalization being a shift in the level (Sec. 2.1). Here we want to show that this same property is shared by our asymmetrically deformed models that hence provide a geometric solution at every order in perturbation.

As we emphasized above, in studying symmetrically deformed WZW models, i.e. those where the deformation operator is written as the product of two currents belonging to the same sector $\mathcal{O}=\lambda J \bar{J}$, one finds that the Lagrangian formulation only corresponds to a small-deformation approximation. For this reason different techniques have been developed so to read the background fields at every order in $\lambda$ but, still, the results are in general only valid at first order in $\alpha^{\prime}$ and have to be modified so to take into account the effect of instanton corrections (. This is not the case for asymmetrically deformed models, for which the background fields in Eqs. (3.25) are exact at all orders in $\mathrm{H}_{a}$ and for which the effect of renormalization only amounts to the usual (for WZW models) shift in the level of the algebra $k \rightarrow k+g^{*}$.

Consider in example the most simple $S U(2)$ case (which we will review in greater detain in Sec. 4.1). In terms of Euler angles the deformed Lagrangian is written as:

$$
\begin{aligned}
S=S_{S U(2)}(\alpha, \beta, \gamma)+\delta S=\frac{k}{4 \pi} \int \mathrm{d}^{2} z \partial \alpha \bar{\partial} \alpha & +\partial \beta \bar{\partial} \beta+\partial \gamma \bar{\partial} \gamma+2 \cos \beta \partial \alpha \bar{\partial} \gamma+ \\
& +\frac{\sqrt{k k_{g}} \mathrm{H}}{2 \pi} \int \mathrm{d}^{2} z(\partial \gamma+\cos \beta \partial \alpha) \bar{I}
\end{aligned}
$$

If we bosonize the right-moving current as $\bar{I}=\bar{\partial} \phi$ and add a standard $U(1)$ term to the action, we get:

$$
\begin{aligned}
& S=S_{S U(2)}(\alpha, \beta, \gamma)+\delta S(\alpha, \beta, \gamma, \phi)+\frac{k_{g}}{4 \pi} \int \mathrm{d}^{2} z \partial \phi \bar{\partial} \phi= \\
& =S_{S U(2)}\left(\alpha, \beta, \gamma+2 \sqrt{\frac{k_{g}}{k}} \mathrm{H} \phi\right)+\frac{k_{g}\left(1-2 \mathrm{H}^{2}\right)}{4 \pi} \int \mathrm{d}^{2} z \partial \phi \bar{\partial} \phi
\end{aligned}
$$

and in particular at the decoupling limit $\mathrm{H} \rightarrow 1 / \sqrt{2}$, corresponding to the $S^{2}$ geometry, the action is just given by $S=S_{S U(2)}\left(\alpha, \beta, \gamma+2 \sqrt{\frac{k_{g}}{k}} \mathrm{H} \phi\right)$. This implies that our (deformed) model inherits all the integrability and renormalization properties of the standard SU(2) WZW model. In other words the threedimensional model with metric and Kalb-Ramond field with $S U(2) \times U(1)$ symmetry and a $U(1)$ gauge field is uplifted to an exact model on the $S U(2)$ group manifold (at least locally): the integrability properties are then a consequence of this hidden $S U(2) \times S U(2)$ symmetry that is manifest in higher dimensions.

The generalization of this particular construction to higher groups is easily obtained if one remarks that the Euler parametrization for the $g \in S U$ (2) 
group representative is written as:

$$
g=e^{\imath \gamma t_{3}} e^{\imath \beta t_{1}} e^{\imath \alpha t_{2}}
$$

where $t_{i}=\sigma_{i} / 2$ are the generators of $\mathfrak{s u}(2)$ ( $\sigma_{i}$ being the usual Pauli matrices). As stated above, the limit deformation corresponds to the gauging of the left action of an abelian subgroup $T \subset S U$ (2). In particular here we chose $T=$ $\left\{h \mid h=e^{l \phi t_{3}}\right\}$, hence it is natural to find (up to the normalization) that:

$$
h(\phi) g(\alpha, \beta, \gamma)=g(\alpha, \beta, \gamma+\phi) \text {. }
$$

The only thing that one needs to do in order to generalize this result to a general group $G$ consists in finding a parametrization of $g \in G$ such as the chosen abelian subgroup appears as a left factor. In example if in $S U(3)$ we want to gauge the $U(1)^{2}$ abelian subgroup generated by $\left\langle\lambda_{3}, \lambda_{8}\right\rangle$ (Gell-Mann matrices), we can choose the following parametrization for $g \in S U(3)$ [Byr97]:

$$
g=e^{\imath \lambda_{8} \phi} e^{\imath \lambda_{3} c} e^{\imath \lambda_{2} b} e^{\imath \lambda_{3} a} e^{\imath \lambda_{5} \vartheta} e^{\imath \lambda_{3} \gamma} e^{\imath \lambda_{2} \beta} e^{\imath \lambda_{3} \alpha} .
$$

The deep reason that lies behind this property (differentiating symmetric and asymmetric deformations) is the fact that not only the currents used for the deformation are preserved (as it happens in both cases), but here their very expression is just modified by a constant factor. In fact, if we write the deformed metric as in Eq. (3.25a) and call $\tilde{K}^{\mu}$ the Killing vector corresponding to the chosen isometry (that doesn't change along the deformation), we see that the corresponding $\tilde{\mathcal{J}}_{\mu}^{(\mathrm{H})}$ current is given by:

$$
\tilde{\mathcal{J}}_{v}^{(\mathrm{H})}=\tilde{K}^{\mu} g_{\mu \nu}^{(\mathrm{H})}=\left(1-2 \mathrm{H}^{2}\right) \tilde{\mathcal{J}}_{v}^{(0)}
$$

The most important consequence (from our point of view) of this integrability property is that the SUGRA action in is actually exact and the only effect of renormalization is the $k \rightarrow k+g^{*}$ shift.

\subsection{Partition functions}

Studying the algebraic structure of marginal deformations we have already stressed that they are completely determined by $O(d, \bar{d})$ pseudo-rotations on the charge lattice corresponding to the deforming operator. This means that a modular invariant partition function is simply obtained once we write the initial WZW one, single out those charges and apply the boost. This proves to be a relatively simple exercise for compact groups but presents technical problems even in the most simple non-compact example $S L(2, \mathbb{R})$ which we will study in greater detail in Sec. 4.2.

$\operatorname{SU}(2)$

Instead of a general construction, for sake of clearness, we can start with the most simple - but showing some general features - example, taking the SU(2) 
group (more extensively studied in Sec. 4.1). Our computation will also include the $S^{2}$ limiting geometry. To fix the ideas, we will consider the case $k_{G}=2$, i.e. a $U(1)$ algebra generated by one right-moving complex fermion. As we've seen in Sec. 2.1 the partition function for the supersymmetric $S U(2)$ model can be written as

$$
Z\left[\begin{array}{l}
a ; h \\
b ; g
\end{array}\right]=\sum_{j, \bar{j}=0}^{(k-2) / 2} M^{j \bar{\jmath}} \chi^{j} \frac{\vartheta\left[\begin{array}{l}
a \\
b
\end{array}\right]}{\eta} \bar{\chi}^{\bar{\jmath}} \frac{\bar{\vartheta}\left[\begin{array}{l}
h \\
g
\end{array}\right]}{\bar{\eta}} .
$$

where the $\chi^{j}$ 's are the characters of bosonic $S U(2)_{k-2},(a, b)$ are the $\mathbb{Z}_{2}$ boundary conditions for the left-moving fermions ${ }^{6}$ and $(h, g)$ those of the right-moving - gauge-sector - ones. We can choose any matrix $M^{j \bar{j}}$ compatible with modular invariance of $S U(2)_{k-2}$. Furthermore, the supersymmetric $S U(2)_{k}$ characters can be decomposed in terms of those of the $N=2$ minimal models:

$$
\chi^{j}(\tau) \vartheta\left[\begin{array}{l}
a \\
b
\end{array}\right](\tau, v)=\sum_{m \in \mathbb{Z}_{2 k}} \mathcal{C}_{m}^{j}\left[\begin{array}{l}
a \\
b
\end{array}\right] \Theta_{m, k}\left(\tau,-\frac{2 v}{k}\right),
$$

where the $N=2$ minimal-model characters, determined implicitly by this decomposition, are given in [Kir88, Dob87, Mat87, RY87].

Our aim is to implement the magnetic deformation in this formalism. The deformation acts as a boost on the left-lattice contribution of the Cartan current of the supersymmetric $S U(2)_{k}$ and on the right current from the gauge sector:

$$
\begin{aligned}
& \Theta_{m, k} \bar{\vartheta}\left[\begin{array}{l}
h \\
g
\end{array}\right]=\sum_{n, \bar{n}} \mathrm{e}^{-\imath \pi g\left(\bar{n}+\frac{h}{2}\right)} q^{\frac{1}{2}\left(\sqrt{2 k} n+\frac{m}{\sqrt{2 k}}\right)^{2}} \bar{q}^{\frac{1}{2}\left(\bar{n}+\frac{h}{2}\right)^{2}} \\
& \longrightarrow \sum_{n, \bar{n}} \mathrm{e}^{-\imath \pi g\left(\bar{n}+\frac{h}{2}\right)} q^{\frac{1}{2}\left[\left(\sqrt{2 k} n+\frac{m}{\sqrt{2 k}}\right) \cosh x+\left(\bar{n}+\frac{h}{2}\right) \sinh x\right]^{2}} \\
& \times \bar{q}^{\frac{1}{2}\left[\left(\bar{n}+\frac{h}{2}\right) \cosh x+\left(\sqrt{2 k} n+\frac{m}{\sqrt{2 k}}\right) \sinh x\right]^{2}} .
\end{aligned}
$$

The boost parameter $x$ is related to the vacuum expectation value of the gauge field as follows:

$$
\cosh x=\frac{1}{1-2 \mathrm{H}^{2}} \text {. }
$$

We observe that, in the limit $\mathrm{H}^{2} \rightarrow \mathrm{H}_{\max }^{2}$, the boost parameter diverges $(x \rightarrow \infty)$, and the following constraints arise:

$$
4(k+2) n+2 m+2 \sqrt{2 k} \bar{n}+\sqrt{2 k} h=0 .
$$

Therefore, the limit is well-defined only if the level of the supersymmetric $S U(2)_{k}$ satisfies a quantization condition:

$$
k=2 p^{2}, p \in \mathbb{Z} \text {. }
$$

\footnotetext{
${ }^{6}$ We have removed the contribution of the fermion associated to $J^{3}$ since it is neutral in the deformation process.
} 
This is exactly the charge quantization condition for the flux of the gauge field, Eq. (4.14). Under this condition, the constraints (3.63) lead to

$$
\begin{aligned}
m+p h & \equiv 0 \quad \bmod 2 p=: 2 p N \\
\bar{n} & =2 p n+N, \quad N \in \mathbb{Z}_{2 p} .
\end{aligned}
$$

Decoupled As a consequence, the $U(1)$ corresponding to the combination of charges orpartition function

thogonal to (3.63) decouples (its radius vanishes), and can be removed. We end up with the following expression for the $S^{2}$ partition function contribution:

$$
Z_{S^{2}}\left[\begin{array}{l}
a ; h \\
b ; g
\end{array}\right]=\sum_{j, \bar{\jmath}} M^{j \bar{\jmath}} \sum_{N \in \mathbb{Z}_{2 p}} \mathrm{e}^{\imath \pi g\left(N+\frac{h}{2}\right)} \mathcal{C}_{p(2 N-h)}^{j}\left[\begin{array}{l}
a \\
b
\end{array}\right] \bar{\chi}^{\bar{\jmath}},
$$

in agreement with the result found in [BJKZ96] by using the coset construction. The remaining charge $N$ labels the magnetic charge of the state under consideration. As a result, the $R$-charges of the left $N=2$ superconformal algebra are:

$$
\mathcal{Q}_{R}=n+\frac{a}{2}-\frac{N-h / 2}{p} \bmod 2 .
$$

We now turn to the issue of modular covariance. Under the transformation $\tau \rightarrow-1 / \tau$, the minimal-model characters transform as:

$\mathcal{C}_{m}^{j}\left[\begin{array}{l}a \\ b\end{array}\right]\left(-\frac{1}{\tau}\right)=\mathrm{e}^{\imath \frac{\pi}{2} a b} \frac{1}{k} \sum_{j^{\prime}=0}^{(k-2) / 2} \sin \left(\frac{\pi(2 j+1)\left(2 j^{\prime}+1\right)}{k}\right) \sum_{m^{\prime} \in \mathbb{Z}_{2 k}} \mathrm{e}^{\imath \pi \frac{m m^{\prime}}{k}} \mathcal{C}_{m^{\prime}}^{j^{\prime}}\left[\begin{array}{c}b \\ -a\end{array}\right](\tau)$.

On the one hand, the part of the modular transformation related to $j$ is precisely compensated by a similar term coming from the transformation of $\bar{\chi} \bar{l}$, in Eq. (3.66). On the other hand, the part of the transformation related to the spin structure $(a, b)$ is compensated by the transformation of the other left-moving fermions in the full heterotic string construction. We can therefore concentrate on the transformation related to the $m$ charge, coming from the transformation of the theta-functions at level $k$. We have

$$
\sum_{N \in \mathbb{Z}_{2 p}} \mathrm{e}^{-\imath \pi g\left(N+\frac{h}{2}\right)} \mathcal{C}_{p(2 N-h)}^{j}\left[\begin{array}{l}
a \\
b
\end{array}\right] \rightarrow \frac{1}{\sqrt{2 k}} \sum_{m^{\prime} \in \mathbb{Z}_{4 p^{2}}} \sum_{N \in \mathbb{Z}_{2 p}} \mathrm{e}^{\frac{\imath \pi}{2}\left(g-\frac{m^{\prime}}{p}\right) h} \mathrm{e}^{2 \imath \pi \frac{N\left(m^{\prime}+p g\right)}{2 p}} \mathcal{C}_{m^{\prime}}^{j}\left[\begin{array}{c}
b \\
-a
\end{array}\right] ;
$$

summing over $N$ in $\mathbb{Z}_{2 p}$ leads to the constraint:

$$
m^{\prime}+p g \equiv 0 \quad \bmod 2 p:=-2 p N^{\prime}, N^{\prime} \in \mathbb{Z}_{2 p}
$$

So we end up with the sum

$$
\mathrm{e}^{-\frac{\imath \pi}{2} h g} \sum_{N^{\prime} \in \mathbb{Z}_{2 p}} \mathrm{e}^{-\imath \pi h\left(N^{\prime}+\frac{g}{2}\right)} \mathcal{C}_{p\left(2 N^{\prime}-g\right)}^{j}\left[\begin{array}{c}
b \\
-a
\end{array}\right]
$$


combining this expression with the modular transformation of the remaining right-moving fermions of the gauge sector, we obtain a modular invariant result.

In a similar way one can check the invariance of the full heterotic string under $\tau \rightarrow \tau+1$.

$\operatorname{SU}(3)$

As it is often the case, the $S U(2)$ example is illuminating but not exhaustive. In this situation this is due to the fact that $U(1)$ is the Cartan torus and at the same time the maximal subgroup. For this reason we need to work out in detail the next non-trivial example, $S U(3)$. The main difference is that there are two nonequivalent construction leading to the same algebraic structure but to the two possible different metrics on the $S U(3) / U(1)^{2} \operatorname{coset}^{7}$.

\section{The Kazama-Suzuki decomposition of SU(3)}

We would like to decompose our WZW model in terms of Kazama-Suzuki (KS) cosets, which are conformal theories with extended $N=2$ superconformal symmetry [KS89b, KS89a].

The simplest of those models are the $N=2$ minimal models that are given by the quotient: $S U(2)_{k-2} \times S O(2)_{1} / U(1)_{k}$, and their characters come from the branching relation:

$$
\chi_{k-2}^{j} \Xi_{2}^{s_{2}}=\sum_{m \in \mathbb{Z}_{2 k}} \mathcal{C}_{m}^{j\left(s_{2}\right)} \frac{\Theta_{m, k}}{\eta} .
$$

For convenience, we write the contribution of the world-sheet fermions in terms of $S O(2 n)_{1}$ characters.

Similarly it is possible to construct an $N=2$ coset CFT from $S U(3)$ [KS89b, KS89a]: ${ }^{8}$

$$
\frac{S U(3)_{k-3} \times S O(4)_{1}}{S U(2)_{k-2} \times U(1)_{3 k}} .
$$

The characters of this theory are implicitly defined by the branching relation:

$$
\chi_{k-3}^{\Lambda} \Xi_{4}^{s_{4}}=\sum_{2 j=0}^{k-2} \sum_{n \in \mathbb{Z}_{6 k}} \mathcal{C}_{j n}^{\Lambda\left(s_{4}\right)} \chi_{k-2}^{j} \frac{\Theta_{n, 3 k}}{\eta} .
$$

Therefore combining the two branching relations, we obtain the decomposition of $S U$ (3) in terms of $N=2$ KS models:

$$
\chi_{k-3}^{\Lambda} \Xi_{4}^{s_{4}} \Xi_{2}^{s_{2}}=\sum_{j, m, n} \mathcal{C}_{j n}^{\Lambda\left(s_{4}\right)} \mathcal{C}_{m}^{j\left(s_{2}\right)} \frac{\Theta_{m, k}}{\eta} \frac{\Theta_{n, 3 k}}{\eta}
$$

\footnotetext{
${ }^{7}$ We have already pointed out in Sec. 3.3 that an asymmetric coset in the mathematical sense in general admits more than one left-invariant metric. The two possible choices for $S U(3) / U(1)^{2}$ will be extensively studied in Sec. 4.5

${ }^{8}$ According to our conventions, the weights of a $U(1)$ at level $k$ are $m^{2} / 4 k, m \in \mathbb{Z}_{2 k}$.
} 
This decomposition goes along the following pattern:

$$
\begin{array}{r}
S U(3)_{k-3} \times S O(8)_{1} \rightarrow \frac{S U(3)_{k-3} \times S O(4)_{1}}{S U(2)_{k-2} \times U(1)_{3 k}} \times \frac{S U(2)_{k-2} \times S O(2)_{1}}{U(1)_{k}} \times \\
\times U(1)_{3 k} \times U(1)_{k} \times S O(2)_{1}
\end{array}
$$

and we shall perform the deformation on the left lattice of $U(1)_{3 k} \times U(1)_{k}$. However the deformation will also act on an appropriate sub-lattice of the right-moving gauge sector. The last $S O(2)_{1}$ factor corresponds to the fermions which are neutral in the process so they won't be considered afterwards.

The gauge sector To construct the model we assume that the gauge sector of the heterotic strings contain an unbroken $S O(6)_{1}$, whose contribution to the partition function is, written in terms of $S O(6)_{1}$ free fermionic characters $\bar{\Xi}_{6}^{s_{6}}$. Since we decompose the characters of the left-moving sector according to eq. (3.76), a natural choice for the action of the deformation in the right-moving gauge sector is to use a similar Kazama-Suzuki decomposition, but for $k=3$, in which case the bosonic CFT is trivial:

$$
S O(8)_{1} \rightarrow \frac{S O(4)_{1}}{S U(2)_{1} \times U(1)_{9}} \times \frac{S U(2)_{1} \times S O(2)_{1}}{U(1)_{3}} \times U(1)_{3} \times U(1)_{1} \times S O(2)_{1}
$$

Since as quoted previously two fermions - the $S O(2)_{1}$ factor - are neutral it is enough that the gauge sector contains an $S O(6)_{1}$ subgroup. To achieve this decomposition, first we decompose the $S O(6)_{1}$ characters in terms of $S O(4)_{1} \times$ $\mathrm{SO}(2)_{1}$ :

$$
\bar{\Xi}_{6}^{\bar{s}_{6}}=\sum_{\bar{s}_{4}, \bar{s}_{2} \in \mathbb{Z}_{4}} C\left[\bar{s}_{6} ; \bar{s}_{4}, \bar{s}_{2}\right] \bar{\Xi}_{4}^{\bar{s}_{4}} \bar{\Xi}_{2}^{\bar{s}_{2}}
$$

where the coefficients of the decomposition $S O(6) \rightarrow S O(4) \times S O(2)$ are either zero or one. And then we perform a coset decomposition for the $S O(4)_{1}$ characters:

$$
\bar{\Xi}_{4}^{\bar{s}_{4}}=\sum_{\ell=0,1} \sum_{u \in \mathbb{Z}_{18}} \bar{\omega}_{\ell u}^{\bar{s}_{4}} \bar{\chi}^{\ell} \frac{\bar{\Theta}_{u, 9}}{\bar{\eta}}
$$

in terms of $S U(2)_{1}$ characters $\bar{\chi}^{\ell}$ and $U(1)$ characters $\bar{\Theta}_{u, 9}$. It defines implicitly the coset characters $\overline{\mathcal{O}}_{\ell u}^{\bar{s}_{4}}$. Then the $S U(2)_{1} \times S O(2)_{1}$ characters are decomposed as:

$$
\bar{\chi}^{\ell} \bar{\Xi}_{2}^{\bar{s}_{2}}=\sum_{v \in \mathbb{Z}_{6}} \bar{\omega}_{v}^{\ell, \bar{s}_{2}} \frac{\bar{\Theta}_{v, 3}}{\bar{\eta}} .
$$

So putting together these branching relations we have the following KazamaSuzuki decomposition for the free fermions of the gauge sector:

$$
\bar{\Xi}_{6}^{\bar{s}_{6}}=\sum_{\bar{s}_{4}, \bar{s}_{2} \in \mathbb{Z}_{4}} \sum_{\ell=0,1} \sum_{u \in \mathbb{Z}_{18}} \sum_{v \in \mathbb{Z}_{6}} C\left[\bar{s}_{6} ; \bar{s}_{4}, \bar{s}_{2}\right] \overline{\boldsymbol{\omega}}_{\ell u}^{\bar{s}_{4}} \overline{\boldsymbol{\omega}}_{v}^{\ell, \bar{s}_{2}} \frac{\bar{\Theta}_{u, 9}}{\bar{\eta}} \frac{\bar{\Theta}_{v, 3}}{\bar{\eta}} .
$$


The deformation Now we are in position to perform the asymmetric deformation adding a magnetic field to the model. The deformation acts on the following combination of left and right theta functions:

$$
\Theta_{n, 3 k} \bar{\Theta}_{u, 9} \times \Theta_{m, k} \bar{\Theta}_{v, 3} .
$$

As for the case of $S U(2)$ [IKOP05a], we have to assume that the level obeys the condition:

$$
\sqrt{\frac{k}{3}}=p \in \mathbb{N}
$$

to be able to reach the geometric coset point in the moduli space of CFT. Then we have to perform $O(2,2, \mathbb{R})$ boosts in the lattices of the $U(1)^{\prime}$ s, mixing the left Cartan lattice of the super-WZW model with the right lattice of the gauge sector. These boosts are parametrized in function of the magnetic fields as:

$$
\cosh \Omega_{a}=\frac{1}{1-2 \mathrm{H}_{a}^{2}}, a=1,2 .
$$

Explicitly we have:

$$
\begin{aligned}
& \sum_{N_{1}, N_{2} \in \mathbb{Z}} q^{3 k\left(N_{1}+\frac{m}{6 k}\right)^{2}} q^{k\left(N_{2}+\frac{n}{2 k}\right)^{2}} \times \sum_{f_{1}, f_{2} \in \mathbb{Z}} \bar{q}^{9\left(f_{1}+\frac{u}{18}\right)^{2}} \bar{q}^{3\left(f_{2}+\frac{v}{6}\right)^{2}} \\
& \rightarrow \sum_{N_{1}, N_{2}, f_{1}, f_{2} \in \mathbb{Z}} q^{9\left[p\left(N_{1}+\frac{m}{18 p^{2}}\right) \cosh \Omega_{1}+\left(f_{1}+\frac{u}{18}\right) \sinh \Omega_{1}\right]^{2}} q^{3\left[p\left(N_{2}+\frac{n}{6 p^{2}}\right) \cosh \Omega_{2}+\left(f_{2}+\frac{v}{6}\right) \sinh \Omega_{2}\right]^{2}} \\
& \times \bar{q}^{9\left[\left(f_{1}+\frac{u}{18}\right) \cosh \Omega_{1}+p\left(N_{1}+\frac{m}{18 p^{2}}\right) \sinh \Omega_{1}\right]^{2}} \bar{q}^{3\left[\left(f_{2}+\frac{v}{6}\right) \cosh \Omega_{2}+p\left(N_{2}+\frac{n}{6 p^{2}}\right) \sinh \Omega_{2}\right]^{2}} .
\end{aligned}
$$

After an infinite deformation, we get the following constraints on the charges:

$$
\begin{gathered}
m=p(18 \mu-u), \mu \in \mathbb{Z}_{p} \\
n=p(6 v-v), v \in \mathbb{Z}_{p}
\end{gathered}
$$

and the $U(1)^{2}$ CFT that has been deformed marginally decouples from the rest and can be safely removed. In conclusion, the infinite deformation gives:

$$
\begin{aligned}
Z_{F_{3}}^{\left(s_{4}, s_{2} ; \bar{s}_{6}\right)}(\tau)= & \sum_{\Lambda} \sum_{j} \sum_{\mu, v \in \mathbb{Z}_{p}} \sum_{\bar{s}_{4}, \bar{s}_{2} \in \mathbb{Z}_{4}} C\left[\bar{s}_{6} ; \bar{s}_{4}, \bar{s}_{2}\right] \\
& \sum_{\ell=0,1} \sum_{u \in \mathbb{Z}_{18}} \sum_{v \in \mathbb{Z}_{6}} \mathcal{C}_{j, p(18 \mu-u)}^{\Lambda\left(s_{4}\right)} \mathcal{C}_{p(6 v-v)}^{j\left(s_{2}\right)} \times \bar{\chi}_{k-3}^{\Lambda} \bar{\omega}_{4 ; \ell u} \bar{s}_{v} \bar{\omega}_{v}^{\ell, \bar{s}_{2}}
\end{aligned}
$$

where the sum over $\Lambda, j$ runs over integrable representations. This is the partition function for the $S U(3) / U(1)^{2}$ coset space. The fermionic charges in the left and right sectors are summed according to the standard rules of Gepner heterotic constructions [Gep88]. The modular properties of this partition function are the same as before the deformation, concerning the $\mathbb{Z}_{4}$ indices of the world-sheet fermions. 


\section{Alternative approach: direct abelian coset}

Here we would like to take a different path, by deforming directly the Cartan lattice of $\mathfrak{s u}_{3}$ without decomposing the left CFT in terms of KS $N=2$ theories. It is possible to perform a generalized (super)parafermionic decomposition of the characters of the $\hat{s}_{3}$ super-algebra at level $k$ (containing a bosonic algebra at level $k-3)$ w.r.t. the Cartan torus:

$$
\chi^{\Lambda}\left(\frac{\vartheta\left[\begin{array}{l}
a \\
b
\end{array}\right]}{\eta}\right)^{\operatorname{dim}(\mathbf{j}) / 2}=\sum_{\lambda \in \mathbf{M}^{*} \bmod k \mathbf{M}} \mathcal{P}_{\lambda}^{\Lambda}\left[\begin{array}{l}
a \\
b
\end{array}\right] \frac{\Theta_{\lambda, k}}{\eta^{\operatorname{dim}(\mathfrak{k})}}
$$

where the theta function of the $\widehat{\mathfrak{s u}}_{3}$ affine algebra reads, for a generic weight $\lambda=m_{i} \lambda_{f}^{i}$ :

$$
\Theta_{\lambda, k}=\sum_{\gamma \in \mathbf{M}+\frac{\lambda}{k}} q^{\frac{k}{2} \kappa(\gamma, \gamma)}=\sum_{N^{1}, N^{2} \in \mathbb{Z}} q^{\frac{k}{2}\left\|N^{1} \alpha_{1}+N^{2} \alpha_{2}+\frac{m_{1} \lambda_{f}^{1}+m_{2} \lambda_{f}^{2}}{k}\right\|^{2}} .
$$

To obtain an anomaly-free model (see the discussion at the beginning of Sec. 3.6) it is natural to associate this model with an abelian coset decomposition of an $S U(3)_{1}$ current algebra made with free fermions of the gauge sector. Thus if the gauge group contains an $S U(3)_{1}$ unbroken factor their characters can be decomposed as:

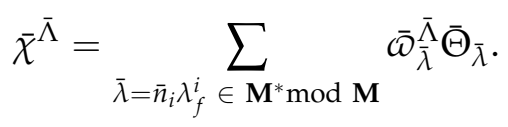

Again we will perform the asymmetric deformation as a boost between the Cartan lattices of the left $\hat{\mathfrak{s u}}_{3}$ algebra at level $k$ and the right $\hat{\mathfrak{s u}}_{3}$ lattice algebra at level one coming from the gauge sector. So after the infinite deformation we will get the quantization condition $\sqrt{k}=p$ and the constraint:

$$
\lambda+p \bar{\lambda}=0 \quad \bmod p \mathbf{M}=: p \mu, \mu \in \mathbf{M}
$$

So we get a different result compared to the Kazama-Suzuki construction. It is so because the constraints that we get at the critical point force the weight lattice of the $\mathfrak{s i u}_{3}$ at level $k$ to be projected onto $p$ times the $\mathfrak{s u} \hat{u}_{3}$ weight lattice at level one of the fermions. This model does not correspond to a Kählerian manifold and should correspond to the $S U$ (3)-invariant metric on the flag space. Indeed with the KS method we get instead a projection onto $p$ times a lattice of $\hat{s i u}_{3}$ at level one which is dual to the orthogonal sub-lattice defined by $\alpha_{1} \mathbb{Z}+\left(\alpha_{1}+2 \alpha_{2}\right) \mathbb{Z}$ - in other words the lattice obtained with the Gell-Mann Cartan generators. In this case it is possible to decompose the model in KS cosets models with $N=2$ superconformal symmetry. ${ }^{9}$

\footnotetext{
${ }^{9}$ For the symmetrically gauged WZW models, this has been studied in [ESY03].
} 


\subsection{The deformation as a gauging}

In this section we want to give an alternative construction for our deformed models, this time explicitly based on an asymmetric WZW gauging. The existence of such a construction is not surprising at all since our deformations can be seen as a generalization of the ones considered in [GK94]. In these terms, just like $J \bar{J}$ (symmetric) deformations lead to gauged WZW models, our asymmetric construction leads to asymmetrically gauged WZW models, which were studied in [QS03].

A point must be stressed here. The asymmetric deformations admit as limit solutions the usual geometric cosets that one would have expected from field theory, as results of a gauging procedure. So, why do we need to go through this somewhat convoluted procedure? The reason lays in the fact that string theory is not the usual point particle field theory. A left and a right sector are present at the same time and they cannot be considered separately if we don't want to introduce anomalies. Now, gauging the left action of a subgroup, i.e. the symmetry $G \sim G H$, which would directly give the geometric coset we are studying, would precisely introduce this kind of problems. Hence we are automatically forced to condider the adjoint action $G \sim H^{-1} G H$ [Wit91]. The key idea then, as it will appear in this section, is that when $G$ is semisimple and written as the product of a group and a copy of its Cartan torus, the left and right action can be chosen such as to act on the two separate sectors and then be equivalent to two left actions.

Instead of a general realization, for sake of clearness, here we will give the explicit construction for the most simple case, the $S U$ (2) model, then introduce a more covariant formalism which will be simpler to generalize to higher groups, in particular for the $S U$ (3) case which we will describe in great detail in the following.

To simplify the formalism we will discuss gauging of bosonic CFTs, and the currents of the gauge sector of the heterotic string are replaced by compact $U(1)$ free bosons. All the results are easily translated into heterotic string constructions.

\section{The SU(2)/U(1) asymmetric gauging}

In this section we want to show how the $S^{2}$ background described in [IKOP05a] can be directly obtained via an asymmetric gauging of the $S U(2) \times U(1)$ WZW model (a similar construction was first obtained in [Joh95]).

Geometric coset as an asymmetric gauging

Consider the WZW model for the group manifold $S U(2)_{k} \times U(1)_{k^{\prime}}$. A parametrisation for the general element of this group which is nicely suited for our purposes is obtained as follows:

$$
g=\left(\begin{array}{ccc}
z_{1} & z_{2} & 0 \\
-\bar{z}_{2} & \bar{z}_{1} & 0 \\
0 & 0 & z_{3}
\end{array}\right)=\left(\begin{array}{c|c}
g_{2} & 0 \\
\hline 0 & g_{1}
\end{array}\right) \in S U(2) \times U(1)
$$

where $g_{1}$ and $g_{2}$ correspond to the $S U(2)$ and $U(1)$ parts respectively and 
$\left(z_{1}, z_{2}, z_{3}\right)$ satisfy:

$$
\operatorname{SU}(2) \times U(1)=\left\{\left.\left(w_{1}, w_{2}, w_{3}\right)|| w_{1}\right|^{2}+\left|w_{2}\right|^{2}=1,\left|w_{3}\right|^{2}=1\right\} \subset \mathbb{C}^{3} .
$$

A possible choice of coordinates for the corresponding group manifold is given by the Euler angles:

$$
\begin{aligned}
& \operatorname{SU}(2) \times U(1) \\
& =\left\{\left(z_{1}, z_{2}, z_{3}\right)=\left(\cos \frac{\beta}{2} e^{l(\gamma+\alpha) / 2}, \sin \frac{\beta}{2} e^{l(\gamma-\alpha) / 2}, e^{\imath \varphi}\right) \mid 0 \leq \beta \leq \pi, 0 \leq \alpha, \beta, \varphi \leq 2 \pi\right\}
\end{aligned}
$$

In order to obtain the coset construction leading to the $S^{2}$ background we define two $U(1) \rightarrow S U(2) \times U(1)$ embeddings as follows:

$$
\begin{aligned}
\epsilon_{L}: U(1) & \rightarrow S U(2) \times U(1) & \epsilon_{R}: U(1) & \rightarrow S U(2) \times U(1) \\
e^{\imath \tau} & \mapsto\left(e^{\imath \tau}, 0,1\right) & e^{\imath \tau} & \mapsto\left(1,0, e^{\imath \tau}\right)
\end{aligned}
$$

so that in terms of the $z$ variables the action of these embeddings boils down to:

$$
\begin{aligned}
g & \mapsto \epsilon_{L}\left(e^{\imath \tau}\right) g \epsilon_{R}\left(e^{\imath \tau}\right)^{-1} \\
\left(w_{1}, w_{2}, w_{3}\right) & \mapsto\left(e^{\imath \tau} w_{1}, e^{\imath \tau} w_{2}, e^{-\imath \tau} w_{3}\right) .
\end{aligned}
$$

This means that we are free to choose a gauge where $w_{2}$ is real or, in Euler coordinates, where $\gamma=\alpha$, the other angular variables just being redefined. To find the background fields corresponding to this gauge choice one should simply write down the Lagrangian where the symmetries corresponding to the two embeddings in (3.95) are promoted to local symmetries, integrate the gauge fields out and then apply a Kaluza-Klein reduction, much in the same spirit as in [IKOP05a].

The starting point is the WZW model, written as:

$$
S_{\text {WZW }}(g)=\frac{k}{4 \pi} \int \mathrm{d} z^{2}\left\langle g_{2}^{-1} \partial g_{2} g_{2}^{-1} \bar{\partial} g_{2}\right\rangle+\frac{k^{\prime}}{4 \pi} \int \mathrm{d} z^{2}\left\langle g_{1}^{-1} \partial g_{1} g_{1}^{-1} \bar{\partial} g_{1}\right\rangle .
$$

Its gauge-invariant generalization is given by:

$$
\begin{aligned}
& S=S_{\mathrm{WZW}} \\
& +\frac{1}{2 \pi} \int \mathrm{d}^{2} z\left[k \bar{A}\left\langle t_{L} \partial g g^{-1}\right\rangle+k^{\prime} A\left\langle t_{R} g^{-1} \bar{\partial} g\right\rangle+\sqrt{k k^{\prime}} A \bar{A}\left(-2+\left\langle t_{L} g t_{R} g^{-1}\right\rangle\right)\right]
\end{aligned}
$$

where $A$ and $\bar{A}$ are the components of the gauge field, and $t_{L}$ and $t_{R}$ are the Lie algebra generators corresponding to the embeddings in (3.95), i.e.

$$
t_{L}=\imath\left(\begin{array}{c|c}
\sigma_{3} & 0 \\
\hline 0 & 0
\end{array}\right), \quad \quad t_{R}=\imath\left(\begin{array}{l|l}
0 & 0 \\
\hline 0 & p
\end{array}\right)
$$


$\sigma_{3}$ being the usual Pauli matrix. For such an asymmetric coset to be anomaly free, one has the following constraint on the embeddings:

$$
k\left\langle t_{L}\right\rangle^{2}=k^{\prime}\left\langle t_{R}\right\rangle^{2} \Longrightarrow k=k^{\prime} p^{2}, \text { with } p \in \mathbb{N} .
$$

If we pass to Euler coordinates it is simple to give an explicit expression for the action:

$$
\begin{aligned}
S(\alpha, \beta, \gamma, \varphi)=\frac{1}{2 \pi} \int \mathrm{d}^{2} z & \frac{k}{4}(\partial \alpha \bar{\partial} \alpha+\partial \beta \bar{\partial} \beta+\partial \gamma \bar{\partial} \gamma+2 \cos \beta \partial \alpha \bar{\partial} \gamma)+\frac{k^{\prime}}{2} \partial \varphi \bar{\partial} \varphi+ \\
& +\imath k(\partial \alpha+\cos \beta \partial \gamma) \bar{A}+\imath k^{\prime} \sqrt{2} \bar{\partial} \varphi A-2 \sqrt{k k^{\prime}} A \bar{A}
\end{aligned}
$$

This Lagrangian is quadratic in $A, \bar{A}$ and the quadratic part is constant so we can integrate these gauge fields out and the resulting Lagrangian is:

$$
\begin{aligned}
S(\alpha, \beta, \gamma, \varphi)=\frac{1}{2 \pi} \int \mathrm{d}^{2} z \frac{k}{4}(\partial \alpha \bar{\partial} \alpha+\partial \beta \bar{\partial} \beta & +\partial \gamma \bar{\partial} \gamma+2 \cos \beta \partial \alpha \bar{\partial} \gamma)+\frac{k^{\prime}}{2} \partial \varphi \bar{\partial} \varphi+ \\
& +\frac{\sqrt{2 k k^{\prime}}}{2}(\partial \alpha+\cos \beta \partial \gamma) \bar{\partial} \varphi .
\end{aligned}
$$

Now, since we gauged out the symmetry corresponding to the $U$ (1) embeddings, this action is redundant. This can very simply be seen by writing the corresponding metric and remarking that it has vanishing determinant:

$$
\operatorname{det} g_{\mu v}=\left|\begin{array}{cccc}
k / 4 & & & \\
& k / 4 & k / 4 \cos \beta & \sqrt{2 k k^{\prime}} / 4 \\
& k / 4 \cos \beta & k / 4 & \sqrt{2 k k^{\prime}} / 4 \cos \beta \\
\sqrt{2 k k^{\prime}} / 4 & \sqrt{2 k k^{\prime}} / 4 \cos \beta & k^{\prime} / 2
\end{array}\right|=0
$$

Of course this is equivalent to say that we have a gauge to fix (as we saw above) and this can be chosen by imposing $\gamma=\alpha$, which leads to the following action:

$$
\begin{array}{r}
S(\alpha, \beta, \varphi)=\frac{1}{2 \pi} \int \mathrm{d}^{2} z \frac{k}{4}(2(1+\cos \beta) \partial \alpha \bar{\partial} \alpha+\partial \beta \bar{\partial} \beta)+\frac{k^{\prime}}{2} \partial \varphi \bar{\partial} \varphi+ \\
+\frac{\sqrt{2 k k^{\prime}}}{2}(1+\cos \beta) \partial \alpha \bar{\partial} \varphi
\end{array}
$$

whence we can read a two dimensional metric by interpreting the $\partial \alpha \bar{\partial} \varphi$ term as a gauge boson and applying the usual Kaluza-Klein reduction. We thus recover the two-sphere as expected:

$$
\mathrm{d} s^{2}=g_{\mu v}-G_{\varphi \varphi} A_{\mu} A_{v}=\frac{k}{4}\left(\mathrm{~d} \beta^{2}+\sin ^{2} \beta \mathrm{d} \alpha^{2}\right)
$$

supported by a (chromo)magnetic field

$$
A=\sqrt{\frac{k}{k^{\prime}}}(1+\cos \beta) \mathrm{d} \alpha
$$




\section{The current formalism}

We now turn to rewrite the above gauging in a more covariant form, simpler to generalize. Since we are interested in the underlying geometry, we will mainly focus on the metric of the spaces we obtain at each step and write these metrics in terms of the Maurer-Cartan currents ${ }^{10}$. As we have already seen, the metric of the initial group manifold is:

$$
\mathrm{d} s^{2}=\frac{k}{2} \sum \mathcal{J}_{i}^{2} \otimes \mathcal{J}_{i}^{2}+\frac{k^{\prime}}{2} \mathcal{I} \otimes \mathcal{I}
$$

where $\left\{\mathcal{J}_{1}, \mathcal{J}_{2}, \mathcal{J}_{3}\right\}$ are the currents of the $S U(2)$ part and $\mathcal{I}$ the $U(1)$ generator. The effect of the asymmetric gauging amounts - at this level - to adding what we can see as an interaction term between the two groups. This changes the metric to:

$$
\mathrm{d} s^{2}=\frac{k}{2} \sum \mathcal{J}_{i}^{2} \otimes \mathcal{J}_{i}^{2}+\frac{k^{\prime}}{2} \mathcal{I} \otimes \mathcal{I}+\sqrt{k k^{\prime}} \mathcal{J}_{3} \otimes \mathcal{I}
$$

Of course if we choose $\left\langle\mathcal{J}_{1}, \mathcal{J}_{2}, \mathcal{J}_{3}, \mathcal{I}\right\rangle$ as a basis we can rewrite the metric in matrix form:

$$
g=\frac{1}{2}\left(\begin{array}{cccc}
k & & & \\
& k & & \\
& & k & \sqrt{k k^{\prime}} \\
& & \sqrt{k k^{\prime}} & k^{\prime}
\end{array}\right)
$$

where we see that the gauging of the axial symmetry corresponds to the fact that the sub-matrix relative to the $\left\{\mathcal{J}_{3}, \mathcal{I}\right\}$ generators is singular:

$$
\left|\begin{array}{cc}
k & \sqrt{k k^{\prime}} \\
\sqrt{k k^{\prime}} & k^{\prime}
\end{array}\right|=0
$$

explicitly this correspond to:

$$
k \mathcal{J}_{3} \otimes \mathcal{J}_{3}+\sqrt{k k^{\prime}} \mathcal{J}_{3} \otimes \mathcal{I}+\sqrt{k k^{\prime}} \mathcal{J}_{3} \otimes \mathcal{I}+k^{\prime} \mathcal{I} \otimes \mathcal{I}=\left(k+k^{\prime}\right) \hat{\mathcal{J}} \otimes \hat{\mathcal{J}}
$$

where

$$
\hat{\mathcal{J}}=\frac{\sqrt{k} \mathcal{J}_{3}+\sqrt{k^{\prime}} \mathcal{I}}{\sqrt{k+k^{\prime}}}
$$

is a normalized current. In matrix terms this corresponds to projecting the interaction sub-matrix on its non-vanishing normalized eigenvector:

$$
\left(\begin{array}{ll}
\sqrt{\frac{k}{k+k^{\prime}}} & \sqrt{\frac{k}{k+k^{\prime}}}
\end{array}\right)\left(\begin{array}{cc}
k & \sqrt{k k^{\prime}} \\
\sqrt{k k^{\prime}} & k^{\prime}
\end{array}\right)\left(\begin{array}{c}
\sqrt{\frac{k}{k+k^{\prime}}} \\
\sqrt{\frac{k}{k+k^{\prime}}}
\end{array}\right)=k+k^{\prime}
$$

\footnotetext{
${ }^{10}$ One of the advantages of just working on the metrics is given by the fact that in each group one can consistently choose left or right currents as a basis. In the following we will consider the group in the initial WZW model as being generated by the left and the dividing group by the right ones.
} 
and the resulting metric in the $\left\langle\mathcal{J}_{1}, \mathcal{J}_{2}, \hat{\mathcal{J}}\right\rangle$ basis is:

$$
\left(\begin{array}{ccc}
k & & \\
& k & \\
& & k+k^{\prime}
\end{array}\right)
$$

This manifold $\mathcal{M}$ (whose metric appears in the action (3.17)) corresponds to an $S^{1}$ fibration (the fiber being generated by $\hat{\mathcal{J}}$ ) over an $S^{2}$ base (generated by $\left.\left\langle\mathcal{J}_{1}, \mathcal{J}_{2}\right\rangle\right)$.

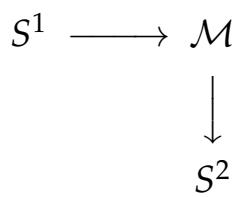

It should now appear natural how to generalize this construction so to include all the points in the moduli space joining the unperturbed and gauged model. The decoupling of the $U(1)$ symmetry (that has been "gauged away") is obtained because the back-reaction of the gauge field (Eq. (3.103)) is such that the interaction sub-matrix is precisely singular. On the other hand we can introduce a parameter that interpolates between the unperturbed and the gauged models so that the interaction matrix now has two non-null eigenvalues, one of which vanishing at the decoupling point.

In practice this is done by adding to the the asymmetrically gauged WZW model an auxiliary $U(1)$ free boson $Y$ at radius $R=\left(k k^{\prime}\right)^{1 / 4}(1 / \sqrt{2} \mathrm{H}-1)^{1 / 2}$. This $U(1)$ is coupled symmetrically to the gauge fields such that the anomaly cancellation condition is still given by (3.101). In particular if we choose the gauge $Y=0$, the metric reads:

$$
\left(\begin{array}{cc}
k & \sqrt{2} \mathrm{H} \sqrt{k k^{\prime}} \\
\sqrt{2} \mathrm{H} \sqrt{k k^{\prime}} & k^{\prime}
\end{array}\right)
$$

which is exactly the model studied above. For a generic value of $\mathrm{H}^{2}$ the two eigenvalues are given by:

$$
\lambda_{2}\left(k, k^{\prime}, \mathrm{H}\right)=\frac{k+k^{\prime} \mp \sqrt{k^{2}+k^{\prime 2}+2\left(4 \mathrm{H}^{2}-1\right) k k^{\prime}}}{2}
$$

so we can diagonalize the metric in the $\left\langle\mathcal{J}_{1}, \mathcal{J}_{2}, \hat{\mathcal{J}}, \hat{\mathcal{J}}\right\rangle$ basis $(\hat{\mathcal{J}}$ and $\hat{\mathcal{J}}$ being the two eigenvectors) and finally obtain:

$$
g=\left(\begin{array}{llll}
k & & & \\
& k & & \\
& & \lambda_{1}\left(k, k^{\prime}, \mathrm{H}\right) & \\
& & & \lambda_{2}\left(k, k^{\prime}, \mathrm{H}\right)
\end{array}\right) .
$$

Of course, in the $\mathrm{H}^{2} \rightarrow 0$ limit we get the initial WZW model and in the $\mathrm{H}^{2} \rightarrow$ $1 / 2$ limit we recover the asymmetrically gauged model, Eq. (3.115).

It is important to remark that the construction above can be directly generalized to higher groups with non-abelian subgroups, at least for the asymmetric coset part. This is what we will further analyse in the next chapter. 



\section{CHAPTER 4}

\section{Applications}

In this chapter we present some of the applications for the construction outlined above. After an analysis of the most simple (compact and noncompact) examples, we describe the near-horizon geometry for the BertottiRobinson black hole, show some new compactifications and see how Horne and Horowitz's black string can be described in this framework and generalized via the introduction of an electric field.

$\mathrm{T}^{\mathrm{H}}$ HE TECHNOLOGY we developed in the previous chapter allows for the construction of a large class of exact string theory backgrounds which is one of the main motivations of the present work. This chapter is devoted to the study of some of the most interesting among them. They can be used to provide new CFT models with clear geometric interpretation (Sec. 4.1 and 4.2), to describe near-horizon geometries of four-dimensional black holes (Sec. 4.3), as laboratories for the study of black holes and black strings (Sec. 4.4) or to provide new physically realistic compactification backgrounds (Sec. 4.5).

\subsection{The two-sphere CFT}

\section{Spacetime fields}

The first deformation that we explicitly consider is the marginal deformation of the SU (2) WZW model. This was first obtained in [KK95] that we will closely follow. It is anyway worth to stress that in their analysis the authors didn't study the point of maximal deformation (which was nevertheless identified as a decompactification boundary) that we will here show to correspond to the 2-sphere $S^{2} \sim S U(2) / U(1)$. Exact CFT's on this background have already obtained in [BK94] and in [Joh95]. In particular the technique used in the latter, namely the asymmetric gauging of an $S U(2) \times U(1)$ WZW model, bears many resemblances to the one we will describe.

Consider a heterotic string background containing the $S U(2)$ group manifold, times some $(1,0)$ superconformal field theory $\mathcal{M}$. The sigma model action is:

$$
S=k S_{S U(2)}(g)+\frac{1}{2 \pi} \int d^{2} z\left\{\sum_{a=1}^{3} \lambda^{a} \bar{\partial} \lambda^{a}+\sum_{n=1}^{g} \tilde{\chi}^{n} \partial \tilde{\chi}^{n}\right\}+S(\mathcal{M}),
$$


Applications

where $\lambda^{i}$ are the left-moving free fermions superpartners of the bosonic $S U(2)$ currents, $\tilde{\chi}^{n}$ are the right-moving fermions of the current algebra and $k S_{S U(2)}(g)$ is the WZW action for the bosonic $S U(2)$ at level $k$. This theory possesses an explicit $S U(2)_{L} \times S U(2)_{R}$ current algebra.

Gauss decomposition for $\operatorname{SU}(2)$

A parametrization of the $S U(2)$ group that is particularly well suited for our purposes is obtained via the so-called Gauss decomposition that we will later generalize to higher groups (see App. B). A general element $g(z, \psi) \in$ $S U(2)$ where $z \in \mathbb{C}$ and $\psi \in \mathbb{R}$ can be written as:

$$
g(z, \psi)=\left(\begin{array}{ll}
1 & 0 \\
z & 1
\end{array}\right)\left(\begin{array}{cc}
1 / \sqrt{f} & 0 \\
0 & \sqrt{f}
\end{array}\right)\left(\begin{array}{cc}
1 & \bar{w} \\
0 & 1
\end{array}\right)\left(\begin{array}{cc}
e^{\imath \psi / 2} & 0 \\
0 & e^{-\imath \psi / 2}
\end{array}\right)
$$

where $w=-z$ and $f=1+|z|^{2}$. In this parametrisation the matrix of invariant one-forms $\Omega=g(z, \psi)^{-1} \mathrm{~d} g(z, \psi)$ which is projected on the Lie algebra generators to give the expression for the Maurer-Cartan one-forms is:

$$
\Omega=\frac{1}{f}\left(\begin{array}{cc}
\bar{z} \mathrm{~d} z-z \mathrm{~d} \bar{z} & -e^{-\imath \psi} \mathrm{d} \bar{z} \\
e^{\imath \psi} \mathrm{d} z & -\bar{z} \mathrm{~d} z+z \mathrm{~d} \bar{z}
\end{array}\right)+\imath\left(\begin{array}{cc}
\mathrm{d} \psi & 0 \\
0 & -\mathrm{d} \psi
\end{array}\right)
$$

(remark that $\Omega$ is traceless and anti-Hermitian since it lives in $\mathfrak{s u}(2)$ ). From $\Omega$ we can easily derive the Cartan-Killing metric on $T_{g} S U(2)_{k}$ as:

$$
\begin{aligned}
\frac{2}{k} \mathrm{~d} s^{2}=\left\langle\Omega^{\dagger} \Omega\right\rangle=-\frac{1}{2 f^{2}}\left(\bar{z}^{2} \mathrm{~d} z^{2}+z^{2} \mathrm{~d} \bar{z}^{2}\right. & \left.-2\left(2+|z|^{2}\right) \mathrm{d} z \mathrm{~d} \bar{z}\right)+ \\
& +\frac{\imath}{f}(z \mathrm{~d} \bar{z}-\bar{z} \mathrm{~d} z) \mathrm{d} \psi+\frac{1}{2} \mathrm{~d} \psi^{2}
\end{aligned}
$$

The left-moving current contains a contribution from the free fermions realizing an $S U(2)_{2}$ algebra, so that the theory possesses (local) $N=(1,0)$ superconformal symmetry.

The marginal deformation is obtained by switching on a magnetic field in the $S U(2)$, introducing the following $(1,0)$-superconformal-symmetry-compatible marginal operator:

$$
\delta S=\frac{\sqrt{k k_{g}} \mathrm{H}}{2 \pi}\left(J^{3}+\lambda^{+} \lambda^{-}\right) \bar{J}
$$

where we have picked one particular current $\bar{J}$ from the gauge sector, generating a $U(1)$ at level $k_{g}$. For instance, we can choose the level-two current: $\bar{J}=i \tilde{\chi}^{1} \tilde{\chi}^{2}$. As a result the solutions to the deformed $\sigma$-model (3.26), (3.33) and 
(3.34) $\operatorname{read}^{1}$ :

$$
\left\{\begin{array}{l}
\frac{1}{k} \mathrm{~d} s^{2}=\frac{\mathrm{d} z \mathrm{~d} \bar{z}}{\left(1+|z|^{2}\right)^{2}}+\left(1-2 \mathrm{H}^{2}\right)\left(\frac{i z \mathrm{~d} \bar{z}-l \bar{z} \mathrm{~d} z}{f}+\mathrm{d} \psi\right)^{2} \\
\mathrm{~d} B=\frac{\imath k}{2} \frac{1}{\left(1+|z|^{2}\right)^{2}} \mathrm{~d} z \wedge \mathrm{d} \bar{z} \wedge \mathrm{d} \psi \\
A=\sqrt{\frac{k}{2 k_{g}}} \mathrm{H}\left(-\frac{i}{f}(\bar{z} \mathrm{~d} z-z \mathrm{~d} \bar{z})+\mathrm{d} \psi\right) .
\end{array}\right.
$$

It can be useful to write explicitly the volume form on the manifold and the Ricci scalar:

$$
\begin{aligned}
\sqrt{\operatorname{det} g} \mathrm{~d} z \wedge \mathrm{d} \bar{z} \wedge \mathrm{d} \psi & =\frac{k}{2} \frac{\sqrt{k\left(1-2 \mathrm{H}^{2}\right)}}{\left(1+|z|^{2}\right)^{2}} \mathrm{~d} z \wedge \mathrm{d} \bar{z} \wedge \mathrm{d} \psi \\
R & =\frac{6+4 \mathrm{H}^{2}}{k}
\end{aligned}
$$

It is quite clear that $\mathrm{H}=\mathrm{H}_{\max }=1 / \sqrt{2}$ is a special point. In general the three-sphere $S U$ (2) can be seen as a non-trivial fibration of $U(1) \sim S^{1}$ as fiber and $S U(2) / U(1) \sim S^{2}$ as base space: the parametrization in (4.7) makes it clear that the effect of the deformation consists in changing the radius of the fiber that naively seems to vanish at $\mathrm{H}_{\max }$. But as we already know the story is a bit different: reparametrizing as in Eq. (3.30):

$$
\psi \rightarrow \frac{\hat{\psi}}{\sqrt{1-2 \mathrm{H}^{2}}}
$$

one is free to take the $\mathrm{H} \rightarrow 1 / \sqrt{2}$ limit where the background fields assume the following expressions:

$$
\left\{\begin{array}{l}
\frac{1}{k} \mathrm{~d} s^{2} \underset{\mathrm{H} \rightarrow 1 / \sqrt{2}}{\longrightarrow} \frac{\mathrm{d} z \mathrm{~d} \bar{z}}{\left(1+|z|^{2}\right)^{2}}+\mathrm{d} \hat{\psi}^{2} \\
F \underset{\mathrm{H} \rightarrow 1 / \sqrt{2}}{\longrightarrow} \sqrt{\frac{k}{4 k_{g}}} \frac{l \mathrm{~d} z \wedge \mathrm{d} \bar{z}}{\left(1+|z|^{2}\right)^{2}} \\
\mathrm{H} \underset{\mathrm{H} \rightarrow 1 / \sqrt{2}}{\longrightarrow} 0
\end{array}\right.
$$

Now we can justify our choice of coordinates: the $(z, \bar{z})$ part of the metric that decouples from the $\psi$ part is nothing else than the Kähler metric for the manifold $\mathbb{C P}^{1}$ (which is isomorphic to $S U(2) / U(1)$ ). In these terms the field strength $F$ is proportional to the Kähler two-form:

$$
F=\imath \sqrt{\frac{k}{k_{g}}} g_{z \bar{z}} \mathrm{~d} z \wedge \mathrm{d} \bar{z} .
$$

\footnotetext{
${ }^{1}$ This type of structure is common to $U(1)$ fibrations over Kähler spaces. In example, the line element for $S^{5}$ which can be seen as a $U(1)$ fiber over $\mathbb{C} P^{2}$ is written as

$$
\mathrm{d} s^{2}=\mathrm{d} s^{2}\left(\mathbb{C} P^{2}\right)+(\mathrm{d} \psi+A)^{2}
$$

where $\mathrm{d} A$ is the Kähler form on $C P^{2}$. We will encounter the same structure again in Sec. 4.5 for $S U(3)$ written as the (principal) fibration $U(1)^{2} \rightarrow S U(3) / U(1)^{2}$.
} 
This begs for a remark. It is simple to show that cosets of the form $G / H$ where $H$ is the maximal torus of $G$ can always be endowed with a Kähler structure. The natural hope is then for this structure to pop up out of our deformations, thus automatically assuring the $N=2$ world-sheet supersymmetry of the model. Actually this is not the case. The Kähler structure is just one of the possible left-invariant metrics that can be defined on a non-symmetric coset (see Sec. 3.3) and the natural generalization of the deformation considered above leads to $\mathbb{C}$-structures that are not Kähler. From this point of view this first example is an exception because $S U(2) / U(1)$ is a symmetric coset since $U(1)$ is not only the maximal torus in $S U(2)$ but also the maximal subgroup. It is nonetheless possible to define an exact CFT on flag spaces but this requires a slightly different construction, already outlined in Sec. 3.6.

We conclude this section observing that the flux of the gauge field on the two-sphere is given by:

$$
\mathcal{Q}=\int_{S^{2}} F=\sqrt{\frac{k}{k_{g}}} \int d \Omega_{2}=\sqrt{\frac{k}{k_{g}}} 4 \pi
$$

However one can argue on general grounds that this flux has to be quantized, e.g. because the two-sphere appears as a factor of the magnetic monopole solution in string theory [KLL99]. This quantization of the magnetic charge is only compatible with levels of the affine $S U(2)$ algebra satisfying the condition:

$$
\frac{k}{k_{g}}=p^{2}, p \in \mathbb{Z}
$$

\section{$4.2 S L(2, \mathbb{R})$}

Anti-de Sitter space in three dimensions is the (universal covering of the) $S L(2, \mathbb{R})$ group manifold. It provides therefore an exact string vacuum with NS background, described in terms of the $S L(2, \mathbb{R})_{k}$ WZW model, where time is embedded in the non-trivial geometry. We will consider it as part of some heterotic string solution such as $\mathrm{AdS}_{3} \times S^{3} \times T^{4}$ with NS three-form field in $\mathrm{AdS}_{3} \times S^{3}$ (near-horizon NS5/F1 background). The specific choice of a background is however of limited importance for our purpose.

The issue of $\mathrm{AdS}_{3}$ deformations has been raised in several circumstances. It is richer ${ }^{2}$ than the corresponding $S^{3}$ owing to the presence of elliptic, hyperbolic or parabolic elements in $S L(2, \mathbb{R})$. The corresponding generators are time-like, space-like or light-like. Similarly, the residual symmetry of a deformed $\mathrm{AdS}_{3}$ has $U(1)$ factors, which act in time, space or light direction.

$S L(2, \mathbb{R})$ marginal symmetric deformations
Marginal symmetric deformations of the $S L(2, \mathbb{R})_{k}$ WZW are driven by bilinears $J \bar{J}$ where both currents are in $S L(2, \mathbb{R})$ and are of the same kind [F9̈4, IKP03]. These break the $S L(2, \mathbb{R})_{\mathrm{L}} \times S L(2, \mathbb{R})_{\mathrm{R}}$ affine symmetry to $U(1)_{\mathrm{L}} \times$ $U(1)_{R}$ and allow to reach, at extreme values of the deformation, gauged $S L(2, \mathbb{R})_{k} / U(1)$

\footnotetext{
${ }^{2}$ As we have already stressed before the Cartan subgroups are not conjugated by inner automorphisms if the group is not simple.
} 
WZW models with an extra free decoupled boson. We can summarize the results as follows:

(a) $J^{3} \bar{J}^{3}$ These are time-like currents (for conventions see App. B) and the corresponding deformations connect $S L(2, \mathbb{R})_{k}$ with $U(1) \times S L(2, \mathbb{R})_{k} /\left.U(1)\right|_{\text {axial or vector }}$. The $U(1)$ factor stands for a decoupled, non-compact time-like free boson $^{3}$. The gauged WZW model $S L(2, \mathbb{R})_{k} /\left.U(1)\right|_{\text {axial }}$ is the cigar (twodimensional Euclidean black hole) obtained by gauging the $g \rightarrow h g h$ symmetry with the $h=\exp i \frac{\lambda}{2} \sigma^{2}$ subgroup, whereas $S L(2, \mathbb{R})_{k} /\left.U(1)\right|_{\text {vector }}$ corresponds to the $g \rightarrow h g h^{-1}$ gauging. This is the trumpet and is T-dual to the cigar ${ }^{4}$. The generators of the affine residual symmetry $U(1)_{\mathrm{L}} \times$ $U(1)_{\mathrm{R}}$ are both time-like (the corresponding Killing vectors are not orthogonal though). For extreme deformation, the time coordinate decouples and the antisymmetric tensor is trade for a dilaton. The isometries are time-translation invariance and rotation invariance in the cigar/trumpet.

(b) $J^{2} \bar{J}^{2}$ The deformation is now induced by space-like currents. So is the residual affine symmetry $U(1)_{L} \times U(1)_{R}$ of the deformed model. Extreme deformation points are T-dual: $U(1) \times S L(2, \mathbb{R})_{k} / U(1)$ where the $U(1)$ factor is space-like, and the $U(1)$ gauging of $S L(2, \mathbb{R})_{k}$ corresponds to $g \rightarrow h g h^{(-1)}$ with $h=\exp -\frac{\lambda}{2} \sigma^{3}$ [DVV92]. The corresponding manifold is (some sector of) the Lorentzian two-dimensional black hole with a non-trivial dilaton.

(c) $\left(J^{1}+J^{3}\right)\left(\bar{J}^{1}+\bar{J}^{3}\right)$ This is the last alternative, with both null currents. The deformation connects $\mathrm{AdS}_{3}$ with $\mathbb{R} \times \mathbb{R}^{1,1}$ plus a dilaton linear in the first factor. The $U(1)_{\mathrm{L}} \times U(1)_{\mathrm{R}}$ left-over current algebra is light-like ${ }^{5}$. Tensorized with an $S U(2)_{k}$ CFT, this background describes the decoupling limit of the NS5/F1 setup [IKP03], where the fundamental strings regularize the strong coupling regime.

Possible choices for the coordinate systems and the resulting fields are reported in App. C.

Our purpose here is to analyze asymmetric deformations of $\mathrm{AdS}_{3}$. Following the similar analysis of the previous section for $S U(2)$, we expect those deformations to preserve a $U(1)_{\mathrm{L}} \times S L(2, \mathbb{R})_{\mathrm{R}}$ symmetry appearing as affine algebra from the sigma-model point of view, and as isometry group for the background. The residual $U(1)_{\mathrm{L}}$ factor can be time-like, space-like or null depending on the current that has been used to perturb the WZW model.

It is worth to stress that some deformations of $\mathrm{AdS}_{3}$ have been studied in the past irrespectively of any conformal sigma-model or string theory analysis. In particular it was observed in [RS98], following [RT83] that the three-

\footnotetext{
${ }^{3}$ The extra bosons are always non-compact.

${ }^{4}$ Actually this statement holds only for the vector coset of the single cover of $S L(2, \mathbb{R})$. Otherwise, from the $n$-th cover of the group manifold one obtains the $n$-th cover of the trumpet [IKP03].

${ }^{5}$ The isometry is actually richer by one (two translations plus a boost), but the extra generator (the boost) is not promoted to an affine symmetry of the sigma-model.
} 
Applications

dimensional ${ }^{6}$ Gödel solution of Einstein equations could be obtained as a member of a one-parameter family of $\mathrm{AdS}_{3}$ deformations that precisely enters the class we discuss here. Gödel space is a constant-curvature Lorentzian manifold. Its isometry group is $U(1) \times S L(2, \mathbb{R})$, and the $U(1)$ factor is generated by a time-like Killing vector. These properties hold for generic values of the deformation parameter. In fact the deformed $\mathrm{AdS}_{3}$ under consideration can be embedded in a seven-dimensional flat space with appropriate signature, as the intersection of four quadratic surfaces. Closed time-like curves as well as high symmetry are inherited from the multi-time maximally symmetric host space. Another interesting property resulting from this embedding is the possibility for changing the sign of the curvature along the continuous line of deformation, without encountering any singular behaviour (see Eq. (4.16)).

It seems natural to generalize the above results to new $\mathrm{AdS}_{3}$ deformations and promote them to exact string backgrounds. Our guideline will be the requirement of a $U(1) \times S L(2, \mathbb{R})$ isometry group, with space-like or light-like $U(1)$ 's.

We will first review the time-like (elliptic) deformation of $\mathrm{AdS}_{3}$ of [RS98] and recently studied from a string perspective in [Isr04]. Hyperbolic (spacelike) and parabolic (light-like) deformations will be analyzed in the following. All these deformations are of the type presented in the previous chapter; further generalizations will be obtained in Sec. 4.4. We show in the following how to implement these deformations as exact marginal perturbations in the framework of the $S L(2, \mathbb{R})_{k}$ WZW model embedded in heterotic string.

\section{Elliptic deformation: magnetic background}

Consider $\mathrm{AdS}_{3}$ in $(t, \rho, \phi)$ coordinates, with metric given in (B.20). In these coordinates, two manifest Killing vectors are $L_{3} \sim \partial_{t}$ and $R_{2} \sim \partial_{\phi}$, time-like and space-like respectively (see App. B, Tab. B.2).

The deformation studied in [RS98] and quoted as "squashed anti de Sitter" reads, in the above coordinates:

$$
\mathrm{d} s^{2}=\frac{L^{2}}{4}\left[\mathrm{~d} \rho^{2}+\cosh ^{2} \rho \mathrm{d} \phi^{2}-\left(1+2 \mathrm{H}^{2}\right)(\mathrm{d} t+\sinh \rho \mathrm{d} \phi)^{2}\right] .
$$

It preserves a $U(1) \times S L(2, \mathbb{R})$ isometry group. The $U(1)$ is generated by the time-like vector $L_{3}$ of one original $S L(2, \mathbb{R})$, while the right-moving $S L(2, \mathbb{R})$ is unbroken (the expressions for the $\left\{L_{3}, R_{1}, R_{2}, R_{3}\right\}$ Killing vectors in Tab. B.2 remain valid at any value of the deformation parameter). The Ricci scalar is constant

$$
R=-\frac{2}{L^{2}}\left(3-2 \mathrm{H}^{2}\right),
$$

while the volume form reads:

$$
\omega_{[3]}=\frac{L^{3}}{8} \sqrt{\left|1+2 \mathrm{H}^{2}\right|} \cosh \rho \mathrm{d} \rho \wedge \mathrm{d} \phi \wedge \mathrm{d} t .
$$

\footnotetext{
${ }^{6}$ In fact, the original Gödel solution is four-dimensional, but the forth space dimension is a flat spectator. In the following, we will systematically refer to the three-dimensional non-trivial factor.
} 
For $\mathrm{H}^{2}=1 / 2$, this deformation coincides with the Gödel metric. It should be stressed, however, that nothing special occurs at this value of the deformation parameter. The properties of Gödel space are generically reproduced at any $\mathrm{H}^{2}>0$.

From a physical point of view, as it stands, this solution is pathological because it has topologically trivial closed time-like curves through each point of the manifold, like Gödel space-time which belongs to this family. Its interest mostly relies on the fact that it can be promoted to an exact string solution, with appropriate NS and magnetic backgrounds. The high symmetry of (4.15), is a severe constraint and, as was shown in [Isr04], the geometry at hand does indeed coincide with the unique marginal deformation of the $S L(2, \mathbb{R})_{k}$ WZW that preserves a $U(1)_{\mathrm{L}} \times S L(2, \mathbb{R})_{\mathrm{R}}$ affine algebra with time-like $U(1)_{\mathrm{L}}$.

It is interesting to observe that, at this stage, the deformation parameter $\mathrm{H}^{2}$ needs not be positive.: (4.15) solves the Einstein-Maxwell-scalar equations [RT83] for any $\mathrm{H}^{2}$. Furthermore, for $\mathrm{H}^{2}<0$, there are no longer closed time-like curves $^{7}$. This statement is based on a simple argument ${ }^{8}$. Consider a time-like curve $x^{\mu}=x^{\mu}(\lambda)$. By definition the tangent vector $\partial_{\lambda}$ is negative-norm, which, by using Eq. (4.15), translates into

$$
\left(\frac{\mathrm{d} \rho}{\mathrm{d} \lambda}\right)^{2}+\cosh ^{2} \rho\left(\frac{\mathrm{d} \phi}{\mathrm{d} \lambda}\right)^{2}-\left(1+2 \mathrm{H}^{2}\right)\left(\frac{\mathrm{d} t}{\mathrm{~d} \lambda}+\sinh \rho \frac{\mathrm{d} \phi}{\mathrm{d} \lambda}\right)^{2}<0 .
$$

If the curve is closed, $\mathrm{d} t / \mathrm{d} \lambda$ must vanish somewhere. At the turning point, the resulting inequality,

$$
\left(2 \mathrm{H}^{2} \sinh ^{2} \rho-1\right)\left(\frac{\mathrm{d} \phi}{\mathrm{d} \rho}\right)^{2}>1
$$

is never satisfied for $\mathrm{H}^{2}<0$, whereas it is for large enough ${ }^{9} \rho$ otherwise.

This apparent regularization of the causal pathology, unfortunately breaks down at the string level. In fact, as we will shortly see, in order to be considered as a string solution, the above background requires a (chromo)magnetic field. The latter turns out to be proportional to $\mathrm{H}$, and becomes imaginary in the range where the closed time-like curves disappear. Hence, at the string level, unitarity is trade for causality. It seems that no regime exists in the magnetic deformation of $\mathrm{AdS}_{3}$, where these fundamental requirements are simultaneously fulfilled.

We now turn to the string realization of the above squashed sphere. In the heterotic backgrounds considered here, of the type $\mathrm{AdS}_{3} \times S^{3} \times T^{4}$, the twodimensional $N=(1,0)$ world-sheet action corresponding to the $\mathrm{AdS}_{3}$ factor

\footnotetext{
${ }^{7}$ As mentioned previously, the geometry at hand can be embedded in a seven-dimensional flat space, with signature $\varepsilon---+++, \varepsilon=\operatorname{sign}\left(-\mathrm{H}^{2}\right)$ [RS98]. This clarifies the origin of the symmetry as well as the presence or absence of closed time-like curves for positive or negative $\mathrm{H}^{2}$.

${ }^{8}$ This argument is local and must in fact be completed by global considerations on the manifold (see [RS98]).

${ }^{9}$ This means $\rho>\rho_{\mathrm{c}}$ where $\rho_{\mathrm{c}}$ is the radius where the norm of $\partial_{\phi}$ vanishes and switches to negative $\left(\left\|\partial_{\phi}\right\|^{2}=L^{2}\left(1-2 \mathrm{H}^{2} \sinh ^{2} \rho\right) / 4\right)$. This never occurs for $\mathrm{H}^{2}<0$.
}

Closed time-like curves in the $S L(2, \mathbb{R})$ elliptic deformation 
is:

$$
S_{S L(2, \mathbb{R})_{k}}=\frac{1}{2 \pi} \int \mathrm{d}^{2} z\left\{\frac{k}{4}(\partial \rho \bar{\partial} \rho-\partial t \bar{\partial} t+\partial \phi \bar{\partial} \phi-2 \sinh \rho \partial \phi \bar{\partial} t)+\eta_{a b} \psi^{a} \bar{\partial} \psi^{b}\right\},
$$

where $\eta_{a b}=\operatorname{diag}(++-), a=1,2,3$ and $\psi^{a}$ are the left-moving superpartners of the $S L(2, \mathbb{R})_{k}$ currents (see Tab. B.2). The corresponding background fields are the metric (Eq. (B.20)) with radius $L=\sqrt{k}$ and the NS B-field:

$$
B=-\frac{k}{4} \sinh \rho \mathrm{d} \phi \wedge \mathrm{d} t
$$

The three-form field strength is $H_{[3]}=\mathrm{d} B=-\frac{2}{\sqrt{k}} \omega_{[3]}$ with $\omega_{[3]}$ displayed in Eq. (B.21).

The asymmetric perturbation that preserves a $U(1)_{\mathrm{L}} \times S L(2, \mathbb{R})_{\mathrm{R}}$ affine algebra with time-like $U(1)_{\mathrm{L}}$ is $\delta S$ given in Eq. (4.5), where $J^{3}$ now stands for the left-moving time-like $S L(2, \mathbb{R})_{k}$ current given in App. B.2, Tab. B.2. This perturbation corresponds to switching on a (chromo)magnetic field, like in the $S U(2)_{k}$ studied in Sec. 4.1. It is marginal and can be integrated for finite values of $\mathrm{H}$, and is compatible with the $N=(1,0)$ world-sheet supersymmetry. The resulting background fields, extracted in the usual manner from the deformed action are the metric (4.15) with radius $L=\sqrt{k}$ and the following gauge field:

$$
A=\mathrm{H} \sqrt{\frac{2 k}{k_{g}}}(\mathrm{~d} t+\sinh \rho \mathrm{d} \phi) .
$$

The NS B-field is not altered by the deformation, (Eq. (4.21)), whereas the three-form field strength depends explicitly on the deformation parameter $\mathrm{H}$, because of the gauge-field contribution:

$$
H_{[3]}=\mathrm{d} B-\frac{k_{G}}{4} A \wedge \mathrm{d} A=-\frac{k}{4}\left(1+2 \mathrm{H}^{2}\right) \cosh \rho \mathrm{d} \rho \wedge \mathrm{d} \phi \wedge \mathrm{d} t .
$$

One can easily check that the background fields (4.15), (4.22) and (4.23) solve the lowest-order equations of motion. Of course the solution we have obtained is exact, since it has been obtained as the marginal deformation of an exact conformal sigma-model. The interpretation of the deformed model in terms of background fields $\left\{G_{a b}, B_{a b}, F_{a b}^{G}\right\}$ receives however the usual higherorder correction summarized by the shift $k \rightarrow k+2$ as we have already explained in Sec. 3.4.

Let us finally mention that it is possible to extract the spectrum and write down the partition function of the above theory [Isr04], since the latter is an exact deformation of the $S L(2, \mathbb{R})_{k}$ WZW model. This is achieved by deforming the associated elliptic Cartan subalgebra. The following picture emerges then from the analysis of the spectrum. The short-string spectrum, corresponding to world-sheets trapped in the "center" of the space-time (for some particular choice of coordinates) is well-behaved, because these world-sheets do not 
feel the closed time-like curves which are "topologically large". On the contrary, the long strings can wrap the closed time-like curves, and their spectrum contains many tachyons. Hence, the caveats of Gödel space survive the string framework, at any value of $\mathrm{H}^{2}>0$. One can circumvent them by slightly deviating from the Gödel line with an extra purely gravitational deformation, driven by $J^{3} \bar{J}^{3}$. This deformation isolates the causally unsafe region, $\rho>\rho_{\mathrm{c}}$ (see [Isr04] for details). It is similar in spirit with the supertube domain-walls of [DFS03] curing the Gödel-like space-times with RR backgrounds.

\section{Hyperbolic deformation: electric background}

\section{The background and its CFT realization}

We will now focus on a different deformation. We use coordinates (B.22) with metric (B.23), where the manifest Killing vectors are $L_{2} \sim \partial_{x}$ (space-like) and $R_{3} \sim \partial_{\tau}$ (time-like) (see App. B.2, Tab. B.3). This time we perform a deformation that preserves a $U(1) \times S L(2, \mathbb{R})$ isometry. The $U(1)$ corresponds to the space-like Killing vector $L_{2}$, whereas the $S L(2, \mathbb{R})$ is generated by $R_{1}, R_{2}, R_{3}$, which are again not altered by the deformation. The resulting metric reads:

$$
\mathrm{d} s^{2}=\frac{L^{2}}{4}\left[\mathrm{~d} r^{2}-\cosh ^{2} r \mathrm{~d} \tau^{2}+\left(1-2 \mathrm{H}^{2}\right)(\mathrm{d} x+\sinh r \mathrm{~d} \tau)^{2}\right] .
$$

The scalar curvature of this manifold is constant

$$
R=-\frac{2}{L^{2}}\left(3+2 \mathrm{H}^{2}\right)
$$

and the volume form

$$
\omega_{[3]}=\frac{L^{3}}{8} \sqrt{\left|1-2 \mathrm{H}^{2}\right|} \cosh ^{2} r \mathrm{~d} r \wedge \mathrm{d} \tau \wedge \mathrm{d} x .
$$

Following the argument of the previous section, one can check whether closed time-like curves appear. Indeed, assuming their existence, the following inequality must hold at the turning point i.e. where $\mathrm{d} t / \mathrm{d} \lambda$ vanishes $(\lambda$ being the parameter that describes the curve):

$$
\left(2 \mathrm{H}^{2}-1\right)\left(\frac{\mathrm{d} x}{\mathrm{~d} r}\right)^{2}>1 .
$$

The latter cannot be satisfied in the regime $\mathrm{H}^{2}<1 / 2$. Notice that the manifold at hand is well behaved, even for negative $\mathrm{H}^{2}$.

Let us now leave aside these questions about the classical geometry, and address the issue of string realization of the above background. As already advertised, this is achieved by considering a world-sheet-supersymmetric marginal deformation of the $S L(2, \mathbb{R})_{k}$ WZW model that implements (chromo)electric field. Such a deformation is possible in the heterotic string at hand:

$$
\delta S=\frac{\sqrt{k k_{G}} \mathrm{H}}{2 \pi} \int \mathrm{d}^{2} z\left(J^{2}+i \psi^{1} \psi^{3}\right) \bar{J}_{G},
$$


( $\bar{J}_{G}$ is any Cartan current of the group $G$ and $J^{2}$ is given in App. B.2, Tab. B.3), and corresponds, as in previous cases, to an integrable marginal deformation. The deformed conformal sigma-model can be analyzed in terms of background fields. The metric turns out to be (4.24), whereas the gauge field and three-form tensor are

$$
\begin{aligned}
A & =\mathrm{H} \sqrt{\frac{2 k}{k_{g}}}(\mathrm{~d} x+\sinh r \mathrm{~d} \tau), \\
H_{[3]} & =\frac{k}{4}\left(1-2 \mathrm{H}^{2}\right) \cosh r \mathrm{~d} r \wedge \mathrm{d} \tau \wedge \mathrm{d} x .
\end{aligned}
$$

As expected, these fields solve the equations of motion.

The background under consideration is a new string solution generated as a hyperbolic deformation of the $S L(2, \mathbb{R})_{k}$ WZW model. In contrast to what happens for the elliptic deformation above, the present solution is perfectly sensible, both at the classical and at the string level.

\section{The spectrum of string primaries}

String primaries for $S L(2, \mathbb{R})$ hyperbolic deformation

The electric deformation of $\mathrm{AdS}_{3}$ is an exact string background. The corresponding conformal field theory is however more difficult to deal with than the one for the elliptic deformation. In order to write down its partition function, we must decompose the $S L(2, \mathbb{R})_{k}$ partition function in a hyperbolic basis of characters, where the implementation of the deformation is well-defined and straightforward; this is a notoriously difficult exercise. On the other hand the spectrum of primaries is known ${ }^{10}$ from the study of the representations of the Lie algebra in this basis (see e.g. [VK91], and [DVV92] for the spectrum of the hyperbolic gauged WZW model, i.e. at the extreme value of the deformation parameter). The part of the heterotic spectrum of interest contains the expression for the primaries of $N=(1,0)$ affine $S L(2, \mathbb{R})$ at purely bosonic level ${ }^{11} k+2$, together with some $U(1)$ from the lattice of the heterotic gauge group:

$$
\begin{aligned}
& L_{0}=-\frac{j(j-1)}{k}-\frac{1}{2}\left(n+\frac{a}{2}\right)^{2}, \\
& \bar{L}_{0}=-\frac{j(j-1)}{k}+\frac{1}{2}\left(\bar{n}+\frac{h}{2}\right)^{2},
\end{aligned}
$$

where the second Casimir of the representation of the $S L(2, \mathbb{R})$ algebra, $-j(j-$ $1)$, explicitly appears. The spectrum contains continuous representations, with $j=\frac{1}{2}+\imath s, s \in \mathbb{R}_{+}$. It also contains discrete representations, with $j \in \mathbb{R}_{+}$, lying within the unitarity range $1 / 2<j<(k+1) / 2$ (see [MO01, Pet90]). In both cases the spectrum of the hyperbolic generator $J^{2}$ is $\mu \in \mathbb{R}$. The expression for the left conformal dimensions, Eq. (4.31), also contains the contribution from

\footnotetext{
${ }^{10}$ In the following we do not consider the issue of the spectral-flow representations. The spectral-flow symmetry is apparently broken by the deformation considered here.

${ }^{11}$ More precisely we consider primaries of the purely bosonic affine algebra with an arbitrary state in the fermionic sector.
} 
the world-sheet fermions associated to the $\imath \psi^{1} \psi^{3}$ current. The sector (R or NS) is labelled by $a \in \mathbb{Z}_{2}$. Note that the unusual sign in front of the lattice is the natural one for the fermions of the light-cone directions. In the expression (4.32) we have similarly the contribution of the fermions of the gauge group, where $h$ labels the corresponding sector.

We are now in position to follow the procedure, familiar from the previous examples: we have to $(i)$ isolate from the left spectrum the lattice of the supersymmetric hyperbolic current $J^{2}+\imath \psi^{1} \psi^{3}$ and (ii) perform a boost between this lattice and the fermionic lattice of the gauge field. We hence obtain the following expressions:

$$
\begin{aligned}
L_{0}=-\frac{j(j-1)}{k}- & \frac{\mu^{2}}{k+2}-\frac{k+2}{2 k}\left(n+\frac{a}{2}+\frac{2 \mu}{k+2}\right)^{2}+ \\
& +\frac{1}{2}\left[\sqrt{\frac{2}{k}}\left(\mu+n+\frac{a}{2}\right) \cosh x+\left(\bar{n}+\frac{h}{2}\right) \sinh x\right]^{2}, \\
\bar{L}_{0}=-\frac{j(j-1)}{k}+ & \frac{1}{2}\left[\left(\bar{n}+\frac{h}{2}\right) \cosh x+\sqrt{\frac{2}{k}}\left(\mu+n+\frac{a}{2}\right) \sinh x\right]^{2} .
\end{aligned}
$$

The relation between the boost parameter $x$ and the deformation parameter $\mathrm{H}^{2}$ is given in Eq. (3.62), as for the case of the $S U(2)_{k}$ deformation. In particular it is worth to remark that the first three terms of (4.33a) correspond to the left weights of the supersymmetric two-dimensional Lorentzian black hole, i.e. the $S L(2, \mathbb{R}) / O(1,1)$ gauged super-WZW model.

This result is less striking that the whole partition function we obtained for the compact $S U(2)$. It is worthwhile to remark that the difference is only due to technical reasons: in principle the very same construction could be applied for the case at hand but it would require the decomposition of the $S L(2, \mathbb{R})$ partition function in terms of hyperbolic characters that at present is not yet known.

\section{Parabolic deformation: the AdS-wave background}

In the deformations encountered in the previous sections one $S L(2, \mathbb{R})$ isometry breaks down to a $U(1)$ generated either by a time-like or by a space-like Killing vector. Deformations which preserve a light-like isometry do also exist and are easily implemented in Poincaré coordinates.

We require that the isometry group is $U(1) \times S L(2, \mathbb{R})$ with a null Killing vector for the $U(1)$ factor. Following the by now familiar for the particular case of light-like residual isometry, we are lead to

$$
\mathrm{d} s^{2}=L^{2}\left[\frac{\mathrm{d} u^{2}}{u^{2}}+\frac{\mathrm{d} x^{+} \mathrm{d} x^{-}}{u^{2}}-2 \mathrm{H}^{2}\left(\frac{\mathrm{d} x^{+}}{u^{2}}\right)^{2}\right] .
$$


The light-like $U(1)$ Killing vector is $L_{1}+L_{3} \sim \partial_{-}$(see App. B.2, Tab. B.4). The remaining $S L(2, \mathbb{R})$ generators are $\left\{R_{1}+R_{3}, R_{1}-R_{3}, R_{2}\right\}$ and remain unaltered after the deformation.

The above deformed anti-de-Sitter geometry looks like a superposition of $\mathrm{AdS}_{3}$ and of a plane wave (whence the AdS-wave name). As usual, the sign of $\mathrm{H}^{2}$ is free at this stage, and $\mathrm{H}^{2}<0$ are equally good geometries. In the nearhorizon region $\left(|u| \gg\left|\mathrm{H}^{2}\right|\right)$ the geometry is not sensitive to the presence of the wave. On the contrary, this plane wave dominates in the opposite limit, near the conformal boundary.

The volume form is not affected by the deformation, and it is still given in (B.27); neither is the Ricci scalar modified:

$$
R=-\frac{6}{L^{2}} \text {. }
$$

Parabolic discrete Notice also that the actual value of $|\mathrm{H}|$ is not of physical significance: it can deformation

always be absorbed into a reparametrization $x^{+} \rightarrow x^{+} /|\mathrm{H}|$ and $x^{-} \rightarrow x^{-}|\mathrm{H}|$. The only relevant values for $\mathrm{H}^{2}$ can therefore be chosen to be $0, \pm 1$.

We now come to the implementation of the geometry (4.34) in a string background. The only option is to perform an asymmetric exactly marginal deformation of the heterotic $S L(2, \mathbb{R})_{k}$ WZW model that preserves a $U(1)_{\mathrm{L}} \times$ $S L(2, \mathbb{R})_{\mathbb{R}}$ affine symmetry. This is achieved by introducing

$$
\delta S_{\text {electric-magnetic }}=-4 \sqrt{k k_{G}} \mathrm{H} \int \mathrm{d}^{2} z\left(J^{1}+J^{3}+i\left(\psi^{1}+\psi^{3}\right) \psi^{2}\right) \bar{J}_{G},
$$

$\left(J^{1}+J^{3}\right.$ is defined in App. B.2, Tab. B.4). The latter perturbation is integrable and accounts for the creation of a (chromo)electromagnetic field

$$
A=2 \sqrt{\frac{2 k}{k_{G}}} \mathrm{H} \frac{\mathrm{d} x^{+}}{u^{2}} .
$$

It generates precisely the deformation (4.34) and leaves unperturbed the NS field, $H_{[3]}=\mathrm{d} B=-\frac{2}{\sqrt{k}} \omega_{[3]}$.

As a conclusion, the $\mathrm{AdS}_{3}$ plus plane-wave gravitational background is described in terms of an exact conformal sigma model, that carries two extra background fields: an NS three-form and an electromagnetic two-form. Similarly to the symmetric parabolic deformation [IKP03], the present asymmetric one can be used to construct a space-time supersymmetric background. The $S L(2, \mathbb{R})_{k}$-CFT treatment of the latter deformation would need the knowledge of the parabolic characters of the affine algebra, not available at present.

\section{Quantum point particles in the AdS-wave background}

Further insights of the physics of the AdS-wave background can be gathered if we look at the motion of point particles. Let us start with the sigma model Lagrangian where we keep the $\mathrm{H}$ parameter explicitly for sake of consistency:

$$
S=\int \mathrm{d} z^{2} \frac{1}{u^{2}} \partial u \bar{\partial} u+\frac{1}{u^{2}} \partial x^{+} \bar{\partial} x^{-}-2 \frac{\mathrm{H}}{u^{2}} \partial x^{-} \bar{\partial} \varphi+\frac{1}{2} \partial \varphi \bar{\partial} \varphi,
$$


where all the fields are function of $\sigma$ and $\tau$. The point particle limit can be obtained if we let the $\sigma$-dependence drop. This leads to:

$$
S_{\text {point }}=\frac{1}{2} \int \mathrm{d} \tau\left\{\frac{1}{u^{2}} \dot{u}^{2}+\frac{1}{u^{2}} \dot{x}^{-} \dot{x}^{+}+\frac{1}{2} \dot{\varphi}^{2}-2 \frac{\mathrm{H}}{u^{2}} \dot{x}^{-} \dot{\varphi}\right\},
$$

where the dot stands for the time derivative. The fourth dimension $\varphi$ was introduced as a fake direction along which perform a Kaluza-Klein reduction. In this framework the same result is obtained if we consider $\varphi$ as an auxiliary variable and then substitute its equation of motion:

$$
\dot{\varphi}=2 \frac{\mathrm{H} \dot{x}^{-}}{2 u^{2}} .
$$

The resulting effective action is then written as:

$$
S_{\text {point }}=\frac{1}{2} \int \mathrm{d} \tau\left\{\frac{1}{u^{2}} \dot{u}^{2}+\frac{1}{u^{2}} \dot{x}^{-} \dot{x}^{+}-\frac{2 \mathrm{H}^{2}}{u^{4}}\left(x^{-}\right)^{2}\right\}
$$

that is exactly the action for a free particle in the 3D AdS-wave metric in Eq. (4.34). Now, out of this we can derive the Hamiltonian:

$$
H_{\text {point }}=\frac{1}{16}\left(c^{2} p_{+}^{2}+2 u^{2}\left(p_{-} p_{+}+p_{u}^{2}\right)\right),
$$

and with the usual rules of quantization this naturally translates to the Laplacian of the AdS-wave geometry:

$$
\triangle=\nabla_{\mu} \nabla^{\mu}=8 \mathrm{H}^{2} \partial_{+}^{2}+4 u^{2} \partial_{+} \partial_{-}-u \partial_{u}+u^{2} \partial_{u}^{2} .
$$

A quantum point particle described by the wave function $\Psi\left(u, x^{-}, x^{+}\right)$must then obey the Klein-Gordon equation:

$$
-\triangle \Psi\left(u, x^{-}, x^{+}\right)=m^{2} \Psi\left(u, x^{-}, x^{+}\right) .
$$

The fact that only the $u$ variable appears explicitly suggests that we can write the solution as:

$$
\left.\Psi\left(u, x^{-}, x^{+}\right)=\int \mathrm{d} p_{-} \mathrm{d} p_{+} e^{\imath\left(p_{-} x^{-}+p_{+} x^{+}\right.}\right) \tilde{\Psi}\left(u, p_{-}, p_{+}\right)
$$

so that the wave equation becomes:

$u^{2} \partial_{u}^{2} \tilde{\Psi}\left(u, p_{-}, p_{+}\right)-u \partial_{u} \tilde{\Psi}\left(u, p_{-}, p_{+}\right)=\left(-m^{2}+8 \mathrm{H}^{2} p_{+}^{2}+4 u^{2} p_{-} p_{+}\right) \tilde{\Psi}\left(u, p_{-}, p_{+}\right)$.

This is a modified Bessel equation whose canonical form is:

$$
z^{2} y^{\prime \prime}(z)+z y^{\prime}(z)-\left(z^{2}+v^{2}\right) y(z)=0
$$

and after some algebra we can write the general solution as:

$$
\tilde{\Psi}\left(u, p_{-}, p_{+}\right)=u I_{v}\left(2 \sqrt{p_{-} p_{+}} u\right) C_{1}\left(p_{-}, p_{+}\right)+u K_{v}\left(2 \sqrt{p_{-} p_{+}} u\right) C_{2}\left(p_{-}, p_{+}\right)
$$

where $C_{1}\left(p_{-}, p_{+}\right)$and $C_{2}\left(p_{-}, p_{+}\right)$are arbitrary functions, $I_{v}(z)$ and $K_{v}(z)$ are modified Bessel functions of the first and second kind and $v=\sqrt{1-m^{2}+8\left(\mathrm{H} p_{+}\right)^{2}}$. 
Applications

\section{Limiting geometries: $\mathrm{AdS}_{2}$ and $\mathrm{H}_{2}$}

We have analyzed in Sec. 4.1 the behaviour of the magnetic deformation of $S U(2)_{k}$, at some critical (or boundary) value of the modulus $H^{2}$, where the background factorizes as $\mathbb{R} \times S^{2}$ with vanishing NS three-form and finite magnetic field. We would like to address this question for the asymmetric deformations of the $S L(2, \mathbb{R})_{k}$ model and show the existence of limiting situations where the geometry indeed factorizes, in agreement with the expectations following the general analysis of Sec. 3.1

What can we expect in the framework of the $S L(2, \mathbb{R})_{k}$ asymmetric deformations? Any limiting geometry must have the generic $U(1) \times S L(2, \mathbb{R})_{k}$ isometry that translates the affine symmetry of the conformal model. If a line decouples, it accounts for the $U(1)$, and the remaining two-dimensional surface must be $S L(2, \mathbb{R})$-invariant. Three different situations may arise: $\mathrm{AdS}_{2}, \mathrm{H}_{2}$ or $\mathrm{dS}_{2}$. Anti de Sitter in two dimensions is Lorentzian with negative curvature; the hyperbolic plane $\mathrm{H}_{2}$ (also called Euclidean anti de Sitter) is Euclidean with negative curvature; de Sitter space is Lorentzian with positive curvature.

Three deformations are available for $\mathrm{AdS}_{3}$ and these have been analyzed in Sec. 4.2. For unitary string theory, all background fields must be real and consequently $\mathrm{H}^{2}>0$ is the only physical regime. In this regime, only the hyperbolic (electric) deformation exhibits a critical behaviour at $\mathrm{H}_{\max }^{2}=1 / 2$. For $\mathrm{H}^{2}<1 / 2$, the deformation at hand is a Lorentzian manifold with no closed time-like curves. When $\mathrm{H}^{2}>1 / 2$, detg $>0$ and two time-like directions appear. At $\mathrm{H}^{2}=\mathrm{H}_{\max }^{2}$, detg vanishes, and this is the signature that some direction indeed decompactifies.

Decompactifying to $\mathrm{AdS}_{2}$
We proceed therefore as in Sec. 3.1, and define a rescaled coordinate in order to keep the decompactifying direction into the geometry and follow its decoupling:

$$
y=\sqrt{\frac{k}{2}\left(\frac{1}{2}-\mathrm{H}^{2}\right)} x .
$$

The metric and volume form now read:

$$
\mathrm{d} s^{2}=\mathrm{d} y^{2}+\frac{k}{4}\left[\mathrm{~d} r^{2}-\left(1+2 \mathrm{H}^{2} \sinh ^{2} r\right) \mathrm{d} \tau^{2}\right]+\sqrt{k\left(1-2 \mathrm{H}^{2}\right)} \sinh r \mathrm{~d} \tau \mathrm{d} y
$$

and

$$
\omega_{[3]}=\frac{k}{4} \cosh r \mathrm{~d} r \wedge \mathrm{d} \tau \wedge \mathrm{d} y .
$$

For $\mathrm{H}^{2}$ close to $\mathrm{H}_{\max }^{2}$, the $y$-direction factorizes

$$
\mathrm{d} s^{2} \underset{\mathrm{H}^{2} \rightarrow \mathrm{H}_{\max }^{2}}{\longrightarrow} \mathrm{d} y^{2}+\frac{k}{4}\left[\mathrm{~d} r^{2}-\cosh ^{2} r \mathrm{~d} \tau^{2}\right] .
$$

The latter expression captures the phenomenon we were expecting:

$$
\mathrm{AdS}_{3} \underset{\mathrm{H}^{2} \rightarrow \mathrm{H}_{\max }^{2}}{\longrightarrow} \mathbb{R} \times \mathrm{AdS}_{2} .
$$


It also shows that the two-dimensional anti de Sitter has radius $\sqrt{k / 4}$ and supports entirely the curvature of the limiting geometry, $R=-8 / k$ (see expression (4.25)).

The above analysis shows that, starting from the $S L(2, \mathbb{R})_{k}$ WZW model, there is a line of continuous exact deformation (driven by a (chromo)electric field) that leads to a conformal model at the boundary of the modulus $\mathrm{H}^{2}$. This model consists of a free non-compact boson times a geometric coset $\mathrm{AdS}_{2} \equiv$ $S L(2, \mathbb{R}) / U(1)$, with a finite electric field:

$$
F=\sqrt{\frac{k}{k_{G}}} \cosh r \mathrm{~d} r \wedge \mathrm{d} \tau
$$

and vanishing NS three-form background. The underlying geometric structure that makes this phenomenon possible is that $\mathrm{AdS}_{3}$ can be considered as a nontrivial $S^{1}$ fibration over an $\mathrm{AdS}_{2}$ base. The radius of the fiber couples to the electric field, and vanishes at $\mathrm{H}_{\max }^{2}$. The important result is that this enables us to promote the geometric coset $\mathrm{AdS}_{2}$ to an exact string vacuum.

We would like finally to comment on the fate of $\mathrm{dS}_{2}$ and $\mathrm{H}_{2}$ geometries, which are both $S L(2, \mathbb{R})$-symmetric. De Sitter and hyperbolic geometries are not expected to appear in physical regimes of string theory unless RamondRamond fields are turned on (see $\mathrm{Ch}$. 7). The $\mathrm{H}_{3}$ sigma-model, for example, is an exact conformal field theory, with imaginary antisymmetric tensor background though [Gaw91, Tes99]. Similarly, imaginary NS background is also required for de Sitter vacua to solve the low-energy equations. It makes sense therefore to investigate regimes with $\mathrm{H}^{2}<0$, where the electric or magnetic backgrounds are indeed imaginary.

The elliptic (magnetic) deformation exhibits a critical behaviour in the region of negative $\mathrm{H}^{2}$, where the geometry does not contain closed time-like curves. The critical behaviour appears at the minimum value $\mathrm{H}_{\min }^{2}=-1 / 2$, below which the metric becomes Euclidean. The vanishing of detg at this point of the deformation line, signals the decoupling of the time direction. The remaining geometry is nothing but a two-dimensional hyperbolic plane $\mathrm{H}_{2}$. It is Euclidean with negative curvature $R=-8 / k$ (see Eq. (4.16) with $L^{2}=k$ ).

All this can be made more precise by introducing a rescaled time coordinate:

$$
T=\sqrt{\frac{k}{2}\left(\frac{1}{2}+\mathrm{H}^{2}\right)} t .
$$

The metric and volume form now read:

$$
\mathrm{d} s^{2}=-\mathrm{d} T^{2}+\frac{k}{4}\left[\mathrm{~d} \rho^{2}+\left(1-2 \mathrm{H}^{2} \sinh ^{2} \rho\right) \mathrm{d} \phi^{2}\right]-\sqrt{k\left(1+2 \mathrm{H}^{2}\right)} \sinh \rho \mathrm{d} \phi \mathrm{d} T
$$

and

$$
\omega_{[3]}=\frac{k}{4} \cosh \rho \mathrm{d} \rho \wedge \mathrm{d} \phi \wedge \mathrm{d} T .
$$


For $\mathrm{H}^{2}$ close to $\mathrm{H}_{\min }^{2}$, the $T$-direction factorizes

$$
\mathrm{d} s^{2} \underset{\mathrm{H}^{2} \rightarrow \mathrm{H}_{\max }^{2}}{\longrightarrow}-\mathrm{d} T^{2}+\frac{k}{4}\left[\mathrm{~d} \rho^{2}+\cosh ^{2} \rho \mathrm{d} \phi^{2}\right] .
$$

The latter expression proves the above statement:

$$
\mathrm{AdS}_{3} \underset{\mathrm{H}^{2} \rightarrow \mathrm{H}_{\text {min }}^{2}}{\longrightarrow} \mathbb{R} \times \mathrm{H}_{2}
$$

and the two-dimensional hyperbolic plane has radius $\sqrt{k / 4}$.

Our analysis finally shows that the continuous line of exactly marginal (chromo)magnetic deformation of the $S L(2, \mathbb{R})$ conformal model has a boundary at $\mathrm{H}^{2}=-1 / 2$ where its target space is a free time-like coordinate times a hyperbolic plane. The price to pay for crossing $\mathrm{H}^{2}=0$ is an imaginary magnetic field, which at $\mathrm{H}^{2}=-1 / 2$ reads:

$$
F=\sqrt{-\frac{k}{k_{G}}} \cosh \rho \mathrm{d} \phi \wedge \mathrm{d} \rho .
$$

The NS field strength vanishes at this point, and the geometric origin of the decoupling at hand is again the Hopf fibration of the $\mathrm{AdS}_{3}$ in terms of an $\mathrm{H}_{2}$.

\section{The $H_{2}$ spectrum}

A $H_{2} \times \mathbb{R}_{t}$ limit geometry can be reached if we allow for negative values of $\mathrm{H}^{2}$ which in turn imply the presence of an imaginary magnetic field. Although this implies that the corresponding string theory is pathological (in example because of unitarity problems), we obtain a perfectly respectable CFT for which we can write, using the same technique as above, a modular-invariant partition function.

The $\mathrm{H}_{2}$ CFT Let us start from the deformed partition function (see [Isr04]). The interesting part for us is:

$$
\begin{aligned}
& \int \mathrm{d}^{2} t Z_{\text {cigar }}\left[\begin{array}{l}
-t_{1} \\
-t_{2}
\end{array}\right] \sum_{N, W, n, \bar{n} \in \mathbb{Z}} e^{i \pi\left(2 N t_{2}+b\left(n+\frac{a}{2}\right)-\delta\left(\bar{n}+\frac{\gamma}{2}\right)\right)} \\
& \times q^{-\left[\frac{\cos \zeta}{\sqrt{k+2}}\left(\frac{N}{2}+\frac{k+4}{2}\left(W+t_{1}\right)+n+\frac{a}{2}\right)+\frac{\sin \zeta}{\sqrt{2}}\left(\bar{n}+\frac{\gamma}{2}\right)\right]^{2}+\frac{k+4}{2(k+2)}\left(n+\frac{a}{2}+\left(W+t_{1}\right)+\frac{N}{k+4}\right)^{2}} \\
& \quad \times \bar{q}^{-\frac{1}{k+4}\left(\frac{N}{2}-\frac{k+4}{2}\left(W+t_{1}\right)\right)^{2}+\left[\frac{\cos \zeta}{\sqrt{2}}\left(\bar{n}+\frac{\gamma}{2}\right)-\frac{\sin \zeta}{\sqrt{k+2}}\left(N+\frac{k+4}{2}\left(W+t_{1}\right)+n+\frac{a}{2}\right)\right]^{2}}
\end{aligned}
$$

where

$$
\cos \zeta=\frac{1}{1+2 \mathrm{H}^{2}}
$$

If we consider $\mathrm{H}^{2}<0$ the trigonometric functions became hyperbolic and there is a critical point $\mathrm{H}^{2}=-1 / 2$ where the boost diverges. Consistency then imposes the following constraint on the charges:

$$
\frac{1}{\sqrt{k+2}}\left(\frac{N}{2}+\frac{k+4}{2}\left(W+t_{1}\right)+n+\frac{a}{2}\right)+\frac{1}{\sqrt{2}}\left(\bar{n}+\frac{\gamma}{2}\right)=0
$$


introducing, for notation convenience

$$
k=2 p^{2}-2
$$

where $p \in \mathbb{R}$ (there is no reason for quantization), the constraint can be rewritten as

$$
N+2\left(p^{2}+1\right)\left(W+t_{1}\right)+2 n+a+p(\bar{n}+2 \gamma)=0
$$

that is equivalent to asking

$$
\left\{\begin{array}{l}
N+2 n+a=Q \in \mathbb{Z} \\
2\left(p^{2}+1\right)\left(W+t_{1}\right)+p(\bar{n}+2 \gamma)=-Q
\end{array}\right.
$$

whence we can rewrite $t_{1}$ as

$$
t_{1}=-\frac{Q+p(2 \bar{n}+\gamma)}{2\left(p^{2}+1\right)}-W
$$

and Eq. (4.61) becomes:

$$
\begin{aligned}
\int \mathrm{d} t_{2} \sum_{N, Q, W, \bar{n} \in \mathbb{Z}} Z_{\text {cigar }}\left[\begin{array}{c}
\frac{Q+2 p(\bar{n}+\gamma / 2)}{2\left(p^{2}+1\right)}+W \\
-t_{2}
\end{array}\right] e^{\imath \pi\left(2 N t_{2}+b \frac{Q-N}{2}-\delta(\bar{n}+\gamma / 2)\right)} \times \\
\times q^{\frac{1}{2\left(p^{2}+1\right)}\left(p \frac{Q-N}{2}-(\bar{n}+\gamma / 2)\right)^{2}} \bar{q}-\frac{1}{2\left(p^{2}+1\right)}\left(\frac{Q+N}{2}+p(\bar{n}+\gamma / 2)\right)^{2}
\end{aligned}
$$

or, introducing the integers $A, B$ as:

$$
\begin{aligned}
& A=Q+N \\
& B=Q-N
\end{aligned}
$$

finally can write the $H^{2}$ partition function as follows:

$$
\begin{aligned}
\int \mathrm{d} t_{2} \sum_{A, B, W, \bar{n} \in \mathbb{Z}} Z_{\text {cigar }}\left[\begin{array}{c}
\frac{A+B+4 p(\bar{n}+\gamma / 2)}{4\left(p^{2}+1\right)}+W \\
-t_{2}
\end{array}\right] e^{\imath \pi\left((A-B) t_{2}+b \frac{B}{2}-\delta(\bar{n}+\gamma / 2)\right)} \times \\
\times q^{\frac{1}{2\left(p^{2}+1\right)}\left(p \frac{B}{2}-(\bar{n}+\gamma / 2)\right)^{2}} \bar{q}^{-\frac{1}{2\left(p^{2}+1\right)}\left(\frac{A}{2}+p(\bar{n}+\gamma / 2)\right)^{2}} .
\end{aligned}
$$

It is intriguing to find that the partition function for the geometric coset $\mathrm{H}_{2}=$ $\mathrm{AdS}_{3} / \mathbb{R}$ is related to the one for the adjoint coset cigar $=\mathrm{AdS}_{3} / \mathbb{R}$. One may wonder if this hints at some operation allowing to pass from the former to the latter, but we will not speculate further in this direction.

\subsection{Near horizon geometry for the Bertotti-Robinson black hole}

The $\mathrm{AdS}_{2} \times S^{2}$ geometry appeared first in the context of Reissner-Nordström black holes. The latter are solutions of Maxwell-Einstein theory in four dimensions, describing charged, spherically symmetric black holes. For a black hole 
of mass $M$ and charge $Q$, the solution reads:

$$
\begin{aligned}
\mathrm{d} s^{2} & =-\left(1-\frac{r_{+}}{r}\right)\left(1-\frac{r_{-}}{r}\right) \mathrm{d} t^{2}+\frac{\mathrm{d} r^{2}}{\left(1-\frac{r_{+}}{r}\right)\left(1-\frac{r_{-}}{r}\right)}+r^{2} \mathrm{~d} \Omega_{2}^{2}, \\
F & =\frac{Q}{r^{2}} \mathrm{~d} t \wedge \mathrm{d} r \quad \text { with } \quad r_{ \pm}=G_{4}\left(M \pm \sqrt{M^{2}-Q^{2}}\right)
\end{aligned}
$$

$r_{+}$and $r_{-}$are the outer and inner horizons, and $G_{4}$ is Newton's constant in four dimensions.

In the extremal case, $r_{+}=r_{-}=r_{0}\left(M^{2}=Q^{2}\right)$, and the metric approaches the $\mathrm{AdS}_{2} \times S^{2}$ geometry in the near-horizon ${ }^{12}$ limit $r \rightarrow r_{0}$. This solution can of course be embedded in various four-dimensional compactifications of string theory, and will be supersymmetric in the extremal case (see e.g. [You99] for a review). In this context we are dealing with some heterotic compactification.

Notice that the $\mathrm{AdS}_{2} \times S^{2}$ geometry also appears in type IIB superstring theory, but with RR backgrounds [FKS95]. The black hole solution is obtained by wrapping D3-branes around 3-cycles of a Calabi-Yau three-fold; in the extremal limit, one obtains the $\mathrm{AdS}_{2} \times S^{2}$ solution, but at the same time the $\mathrm{CY}$ moduli freeze to some particular values. A hybrid Green-Schwarz sigmamodel action for this model has been presented in [BBH ${ }^{+} 00$ ] (see also [Ver04] for $\left.A d S_{2}\right)$. The interest for $A d S_{2} \times S^{2}$ space-time is motivated by the fact that it provides an interesting simplified laboratory for AdS/CFT correspondence [Ma198]. In the present case the dual theory should correspond to some superconformal quantum mechanics [BPS98, C+98, GT99, CCKM01].

\section{The spectrum}

As a first step in the computation of the $\mathrm{AdS}_{2} \times S^{2}$ string spectrum, we must determine the spectrum of the $\mathrm{AdS}_{2}$ factor, by using the same limiting procedure as in Sec. 4.1 for the sphere. The spectrum of the electrically deformed

Boost on the spectrum of $\mathrm{AdS}_{2}$ primaries

$\mathrm{AdS}_{3}$, is displayed in Eqs. (4.33a) and (4.33b). The $\mathrm{AdS}_{2}$ limit is reached for $\cosh x \rightarrow \infty$, which leads to the following constraint on the charges of the primary fields:

$$
\bar{n}+\frac{h}{2}+\sqrt{\frac{2}{k}}\left(\mu+n+\frac{a}{2}\right)=0 .
$$

In contrast with the $S^{2}$ case, since $\mu$ is any real number - irrespectively of the kind of $S L(2, \mathbb{R})$ representation - there is no extra quantization condition for the level to make this limit well-defined. In this limit, the extra $U(1)$ decompactifies as usual and can be removed. Plugging the constraint (4.73) in the

\footnotetext{
${ }^{12}$ With the near-horizon coordinates $U=\left(1-r_{0} / r\right)^{-1}$ and $T=t / r_{0}$, the near-horizon geometry is

$$
\mathrm{d} s^{2}=r_{0}^{2}\left(-\frac{\mathrm{d} T^{2}}{U^{2}}+\frac{\mathrm{d} U^{2}}{U^{2}}+\mathrm{d} \Omega_{2}^{2}\right) .
$$
}

Both $\mathrm{AdS}_{2}$ and $S^{2}$ factors have the same radius $r_{0}$. 
expressions for the dimensions of the affine primaries, we find

$$
\begin{aligned}
& L_{0}=-\frac{j(j-1)}{k}-\frac{1}{2}\left(\bar{n}+\frac{h}{2}\right)^{2}-\frac{1}{2}\left(n+\frac{a}{2}\right)^{2}, \\
& \bar{L}_{0}=-\frac{j(j-1)}{k} .
\end{aligned}
$$

In addition to the original $\mathrm{AdS}_{3}$ spectrum, Eqs. (4.31) and (4.32), the rightmoving part contain an extra fermionic lattice describing the states charged under the electric field. Despite the absence of $N=2$ superconformal symmetry due to the Lorentzian signature, the theory has a "fermion-number" left symmetry, corresponding to the current:

$$
J=\imath \psi^{1} \psi^{3}+\frac{2}{k}\left(J^{2}+\imath \psi^{1} \psi^{3}\right)
$$

The charges of the primaries (4.74) are

$$
\mathcal{Q}_{F}=n+\frac{a}{2}-\sqrt{\frac{2}{k}}\left(\bar{n}+\frac{h}{2}\right) .
$$

\section{$\mathrm{AdS}_{2} \times S^{2} \times \mathcal{M}$ and space-time supersymmetry}

Let us now consider the complete heterotic string background which consists of the $\mathrm{AdS}_{2} \times S^{2}$ space-time times an $N=2$ internal conformal field theory $\mathcal{M}$, that we will assume to be of central charge $\hat{c}=6$ and with integral $R$ charges. Examples of thereof are toroidal or flat-space compactifications, as well as Gepner models [Gep88].

The levels $k$ of $S U(2)$ and $\hat{k}$ of $S L(2, \mathbb{R})$ are such that the string background is critical:

$$
\hat{c}=\frac{2(k-2)}{k}+\frac{2(\hat{k}+2)}{\hat{k}}=4 \Longrightarrow k=\hat{k}
$$

Supersymmetry and level quantization for $\mathrm{AdS}_{2} \times S^{2}$

This translates into the equality of the radii of the corresponding $S^{2}$ and $\mathrm{AdS}_{2}$ factors, which is in turn necessary for supersymmetry. Furthermore, the charge quantization condition for the two-sphere (Sec. 4.1) imposes a further restriction on the level to $k=2 p^{2}, p \in \mathbb{N}$.

In this system the total fermionic charge is

$$
\mathcal{Q}=n+\frac{a}{2}-\frac{N-h / 2}{p}+n^{\prime}+\frac{a}{2}-\frac{\bar{n}^{\prime}+h / 2}{p}+\mathcal{Q}_{\mathcal{M}}
$$

Hence, assuming that the internal $N=2$ charge $\mathcal{Q}_{\mathcal{M}}$ is integral, further constraints on the electromagnetic charges of the theory are needed in order to achieve space-time supersymmetry. Namely, we must only keep states such that

$$
N+\bar{n}^{\prime}=0 \bmod p
$$


This projection is some kind of generalization of Gepner models. Usually, such a projection is supplemented in string theory by new twisted sectors. We then expect that, by adding on top of this projection the usual GSO projection on odd fermion number, one will obtain a space-time supersymmetric background. However, the actual computation would need the knowledge of hyperbolic coset characters of $S L(2, \mathbb{R})$ (i.e. Lorentzian black-hole characters), and of their modular properties. We can already observe that this "Gepner-like" orbifold keeps only states which are "dyonic" with respect to the electromagnetic field background. Notice that, by switching other fluxes in the internal theory $\mathcal{M}$ one can describe more general projections.

\subsection{The three-dimensional black string revisited}

The $\mathrm{AdS}_{3}$ moduli space contains black hole geometries. This has been known since the most celebrated of them - the two-dimensional $S L(2, \mathbb{R}) / U(1)$ black hole - was found by Witten [Wit91, DVV92]. Generalisations of these constructions to higher dimensions have been considered in [HH92, Ger95, Hor92, KT94]. The three-dimensional black string [HH92, HHS92, HW93] has attracted much attention, for it provides an alternative to the Schwarzschild black hole in three-dimensional asymptotically flat geometries ${ }^{13}$. In this section we want to show how this black string can be interpreted in terms of marginal deformations of $S L(2, \mathbb{R})$, which will enable us to give an expression for its string primary states.

The threedimensional black string as a current-current deformation

In [HH92] the black string was obtained as an $(S L(2, \mathbb{R}) \times \mathbb{R}) / \mathbb{R}$ gauged model. More precisely, expressing $g \in S L(2, \mathbb{R}) \times \mathbb{R}$ as:

$$
g=\left(\begin{array}{ccc}
a & u & 0 \\
-v & b & 0 \\
0 & 0 & e^{x}
\end{array}\right)
$$

the left and right embeddings of the $\mathbb{R}$ subgroup are identical and given by:

$$
\begin{aligned}
& \epsilon_{L / R}: \mathbb{R} \rightarrow S L(2, \mathbb{R}) \times \mathbb{R} \\
& \lambda \mapsto\left(\begin{array}{ccc}
\mathrm{e}^{\frac{1}{\sqrt{\lambda^{2}+2}}} & 0 & 0 \\
0 & \mathrm{e}^{-\frac{1}{\sqrt{\lambda^{2}+2}}} & 0 \\
0 & 0 & \mathrm{e}^{\frac{\lambda}{\sqrt{\lambda^{2}+2}}}
\end{array}\right) .
\end{aligned}
$$

From the discussion in Sec. 3.1, we see that performing this gauging is just one of the possible ways to recover the $J^{2} \bar{J}^{2}$ symmetrically deformed $S L(2, \mathbb{R})$ geometry. More specifically, since the gauged symmetry is axial $(g \rightarrow h g h)$, it corresponds (in our notation) to the $\kappa_{2}<1$ branch of the deformed geometry in Eq. $(\mathrm{C} .5 \mathrm{a})^{14}$. One can find a coordinate transformation allowing to pass

\footnotetext{
${ }^{13}$ Remember that the no hair theorem doesn't hold in three dimensions [Isr67, Heu98, GIS02].

${ }^{14}$ The $R \gtrless 1$ convention is not univocal in literature.
} 


\begin{tabular}{|c|c|c|c|c|c|c|}
\hline$\mu$ & name & $\mathrm{d} t^{2}$ & $\mathrm{~d} x^{2}$ & $\mathrm{~d} r^{2}$ & range & CFT interpretation \\
\hline \hline \multirow{3}{*}{$\mu^{2}>1$} & $\left(c^{+}\right)$ & - & + & + & $r>\mu^{2}$ & $J^{3} \bar{J}^{3}, \kappa_{3}>1$ \\
\cline { 2 - 6 } & $\left(b^{+}\right)$ & - & - & - & $1<r<\mu^{2}$ & \\
\cline { 2 - 6 } & $\left(a^{+}\right)$ & + & - & + & $0<r<1$ & $J^{3} \bar{J}^{3}, \kappa_{3}<1$ \\
\hline \hline \multirow{3}{*}{$\mu^{2}<1$} & $\left(a^{-}\right)$ & + & - & + & $0<r<\mu^{2}$ & \multirow{2}{*}{$J^{2} \bar{J}^{2}, \kappa_{2}<1$} \\
\cline { 2 - 5 } & $\left(b^{-}\right)$ & - & + & - & $\mu^{2}<r<1$ & \multirow{2}{*}{} \\
\cline { 2 - 5 } & $\left(c^{-}\right)$ & - & + & + & $r>1$ & \multicolumn{1}{|c}{} \\
\hline
\end{tabular}

Table 4.1: Signature for the black-string metric as a function of $r$, for $\mu^{2} \gtrless 1$.

from the usual black-string solution

$$
\left\{\begin{array}{l}
\mathrm{d} s^{2}=\frac{k}{4}\left[-\left(1-\frac{1}{r}\right) \mathrm{d} t^{2}+\left(1-\frac{\mu^{2}}{r}\right) \mathrm{d} x^{2}+\left(1-\frac{1}{r}\right)^{-1}\left(1-\frac{\mu^{2}}{r}\right)^{-1} \frac{\mathrm{d} r^{2}}{r^{2}}\right] \\
H=\frac{k}{4} \frac{\mu}{r} \mathrm{~d} r \wedge \mathrm{d} x \wedge \mathrm{d} t \\
\mathrm{e}^{2 \Phi}=\frac{\mu}{r}
\end{array}\right.
$$

to our (local) coordinate system, Eq. (C.5). The attentive reader might now be puzzled by this equivalence between a one-parameter model such as the symmetrically deformed model and a two-parameter one such as the black string in its usual coordinates (in Eqs. (4.83) we redefined the $r$ coordinate as $r \rightarrow r / M$ and then set $\mu=Q / M$ with respect to the conventions in [HH92]). A point that it is interesting to make here is that although, out of physical considerations, the black string is usually described in terms of two parameters (mass and charge), the only physically distinguishable parameter is their ratio

Single physical parameter for the black string $\mu=Q / M$ that coincides with our $\kappa_{2}$ parameter. In the next section we will introduce a different (double) deformation, this time giving rise to a black hole geometry depending on two actual parameters (one of which being related to an additional electric field).

As we remarked above, the axial gauging construction only applies for $\mu<1$, while, in order to obtain the other $\kappa_{2}>1$ branch of the $J^{2} \bar{J}^{2}$ deformation, one should perform a vector gauging. On the other hand, this operation, that would be justified by a CFT point of view, is not natural when one takes a more geometrical point of view and writes the black string metric as in Eq. (4.83). In the latter, one can study the signature of the metric as a function of $r$ in the two regions $\mu^{2} \gtrless 1$, and find the physically sensible regions (see Tab. 4.1).

Our observations are the following:

- The $\mu^{2}<1$ branch always has the correct $(-,+,+)$ signature for any value of $r$, with the two special values $r=1$ and $r=\mu^{2}$ marking the presence of the horizons that hide the singularity in $r=0$.

- The $\mu^{2}>1$ branch is different. In particular we see that there are two regions: $\left(a^{+}\right)$for $0<r<1$ and $\left(c^{+}\right)$for $r>\mu^{2}$ where the signature is that of a physical space. 
A fact deserves to be emphasized here: one should notice that while for $\mu^{2}<1$ we obtain three different regions of the same space, for $\mu^{2}>1$ what we show in Tab. 4.1 really are three different spaces and the proposed ranges for $r$ are just an effect of the chosen parameterization. The $\left(a^{+}\right), \kappa_{3}<1$ and $\left(c^{+}\right), \kappa_{3}>1$ branches are different spaces and not different regions of the same one and one can choose in which one to go when continuing to $\mu>1$.

But there is more. The $\mu^{2}>1$ region is obtained via an analytic continuation with respect to the other branch, and this analytic continuation is precisely the one that interchanges the roles of the $J^{2}$ and the $J^{3}$ currents. As a result, we pass from the $J^{2} \bar{J}^{2}$ line to the $J^{3} \bar{J}^{3}$ line. More precisely the $\left(c^{+}\right)$region describes the "singular" $\kappa_{3}>1$ branch of the $J^{3} \bar{J}^{3}$ deformation (i.e. the branch that includes the $r=0$ singularity) and the $\left(a^{+}\right)$region describes the regular $\kappa_{3}<1$ branch that has the cigar geometry as $\kappa_{3} \rightarrow 0$ limit. Also notice that the regions $r<0$ have to be excluded in order to avoid naked singularities (of the type encountered in the Schwarzschild black hole with negative mass). The black string described in [HH92] covers the regions $\left(a^{-}\right),\left(b^{-}\right),\left(c^{-}\right),\left(a^{+}\right)$.

Our last point concerns the expectation of the genuine $\mathrm{AdS}_{3}$ geometry as a zero-deformation limit of the black-string metric, since the latter turns out to be a marginal deformation of $\mathrm{AdS}_{3}$ with parameter $\mu$. The straightforward approach consists in taking the line element in Eq. (4.83) for $\mu=1$. It is then puzzling that the resulting extremal black-string geometry is not $\mathrm{AdS}_{3}$. This apparent paradox is solved by carefully looking at the coordinate transformations that relate the black-string coordinates $(r, x, t)$ to either the Euler coordinates $(\rho, \phi, \tau)$ (B.13) for the $J^{3} \bar{J}^{3}$ line, or the hyperbolic coordinates $(y, x, t)$ for the $J^{2} \bar{J}^{2}$ line. These transformations are singular at $\mu=1$, which therefore corresponds neither to $\kappa_{3}=1$ nor to $\kappa_{2}=1$. Put differently, $\mu=1$ is not part of a continuous line of deformed models but marks a jump from the $J^{2} \bar{J}^{2}$ to the $J^{3} \bar{J}^{3}$ lines.

The extremal black-string solution is even more peculiar. Comparing Eqs. (4.83) at $\mu=1$ to Eqs. (C.6), which describe the symmetrically null-deformed $S L(2, \mathbb{R})$, we observe that the two backgrounds at hand are related by a coordinate transformation, provided $v=-1$.

The black string background is therefore entirely described in terms of $S L(2, \mathbb{R})$ marginal symmetric deformations, and involves all three of them. The null deformation appears, however, for the extremal black string only and at a negative value of the parameter $v$. The latter is the density of fundamental strings, when the deformed $\mathrm{AdS}_{3}$ is considered within the NS5/F1 system. This might be one more sign pointing towards a Gregory-Laflamme instability in the black string [GL93].

Notice finally that expressions (4.83) receive $1 / k$ corrections. Those have been computed in [Sfe93]. Once taken into account, they contribute in making the geometry smoother, as usual in string theory.

\section{An interesting mix}

A particular kind of asymmetric deformation is what we will call in the following double deformation [KK95, Isr04]. At the Lagrangian level this is obtained 
by adding the following marginal perturbation to the WZW action:

$$
\delta S=\delta \kappa^{2} \int \mathrm{d}^{2} z J \bar{J}+\mathrm{H} \int \mathrm{d}^{2} z J \bar{I}
$$

$J$ is a holomorphic current in the group, $\bar{J}$ the corresponding anti-holomorphic current and $\bar{I}$ an external (to the group) anti-holomorphic current (i.e. in the right-moving heterotic sector for example). A possible way to interpret this operator consists in thinking of the double deformation as the superposition of a symmetric - or gravitational - deformation (the first addend) and of an antisymmetric one - the electromagnetic deformation. This mix is consistent because if we perform the $\kappa$ deformation first, the theory keeps the $U(1) \times U(1)$ symmetry generated by $J$ and $\bar{J}$ that is needed in order to allow for the $\mathrm{H}$ deformation. Following this trail, we can read off the background fields corresponding to the double deformation by using at first one of the methods outlined in Sec. 3.1 and then applying the Kaluza-Klein reduction to the resulting background fields.

The final result consists in a metric, a three-form, a dilaton and a gauge field. It is in general valid at any order in the deformation parameters $\kappa$ and $\mathrm{H}$ but only at leading order in $\alpha^{\prime}$ due to the presence of the symmetric part.

Double deformations of $\mathrm{AdS}_{3}$ where $J$ is the time-like $J^{3}$ operator have been studied in [Isr04]. It was there shown that the extra gravitational deformation allows to get rid of the closed time-like curves, which are otherwise present in the pure $J^{3}$ asymmetric deformation (Eq. (4.15)) - the latter includes Gödel space. Here, we will focus instead on the case of double deformation generated by space-like operators, $J^{2}$ and $\bar{J}^{2}$.

\section{The hyperbolic double deformation}

In order to follow the above prescription for reading the background fields in the double-deformed metric let us start with the fields in Eqs. (C.5). We can introduce those fields in the sigma-model action. Infinitesimal variation of the latter with respect to the parameter $\kappa^{2}$ enables us to reach the following expressions for the chiral currents $J_{\mathcal{K}}^{2}(z)$ and $\bar{J}_{\mathcal{K}}^{2}(\bar{z})$ at finite values of $\kappa^{2}$ :

$$
\begin{aligned}
J_{\mathcal{K}}^{2}(z) & =\frac{1}{\cos ^{2} t+\kappa^{2} \sin ^{2} t}\left(\cos ^{2} t \partial \psi-\sin ^{2} t \partial \varphi\right), \\
\bar{J}_{\mathcal{K}}^{2}(\bar{z}) & =\frac{1}{\cos ^{2} t+\kappa^{2} \sin ^{2} t}\left(\cos ^{2} t \partial \psi+\sin ^{2} t \partial \varphi\right) .
\end{aligned}
$$

Note in particular that the corresponding Killing vectors (that clearly are $\partial_{\varphi}$ and $\partial_{\psi}$ ) are to be rescaled as $L_{2}=\frac{1}{\kappa^{2}} \partial_{\psi}-\partial_{\varphi}$ and $R_{2}=\frac{1}{\kappa^{2}} \partial_{\psi}+\partial_{\varphi}$. Once the currents are known, one has to apply the construction sketched in Sec. 3.2 and

A two-parameter charged black string 
write the background fields as follows:

$$
\left\{\begin{array}{l}
\frac{1}{k} \mathrm{~d} s^{2}=-\mathrm{d} t^{2}+\cos ^{2} t \frac{\left(\kappa^{2}-2 \mathrm{H}^{2}\right) \cos ^{2} t+\kappa^{4} \sin ^{2} t}{\Delta_{\kappa}(t)^{2}} \mathrm{~d} \psi^{2}-4 \mathrm{H}^{2} \frac{\cos ^{2} t \sin ^{2} t}{\Delta_{\kappa}(t)^{2}} \mathrm{~d} \psi \mathrm{d} \varphi+ \\
+\sin ^{2} t \frac{\cos ^{2} t+\left(\kappa^{2}-2 \mathrm{H}^{2}\right) \sin ^{2} t}{\Delta_{\kappa}(t)^{2}} \mathrm{~d} \varphi^{2} \\
\frac{1}{k} B=\frac{\kappa^{2}-2 \mathrm{H}^{2}}{\kappa^{2}} \frac{\cos ^{2} t}{\Delta_{\kappa}(t)} \mathrm{d} \varphi \wedge \mathrm{d} \psi \\
F=2 \mathrm{H} \sqrt{\frac{2 k}{\kappa_{g}} \frac{\sin (2 t)}{\Delta_{\kappa}(t)^{2}}}\left(\kappa^{2} \mathrm{~d} \psi \wedge \mathrm{d} t+\mathrm{d} t \wedge \mathrm{d} \varphi\right) \\
\mathrm{e}^{-\Phi}=\frac{\sqrt{\kappa^{2}-2 \mathrm{H}^{2}}}{\Delta_{\kappa}(t)}
\end{array}\right.
$$

where $\Delta_{\kappa}(t)=\cos ^{2} t+\kappa^{2} \sin ^{2} t$ as in App. C. In particular the dilaton, that can be obtained by imposing the one-loop beta equation is proportional to the ratio of the double deformed volume form and the $\mathrm{AdS}_{3}$ one.

A first observation about the above background is in order here. The electric field is bounded from above since $\mathrm{H}^{2} \leq \frac{\kappa^{2}}{2}$. As usual in string theory, tachyonic instabilities occur at large values of electric or magnetic fields, which is just a way of interpreting the decompactification boundary value for the deformation parameter. At the critical value of $\mathrm{H}$, one dimension degenerates and the $B$-field vanishes. We are left with a two-dimensional space (with nonconstant curvature) plus electric field.

The expression (4.87) here above of the metric provides only a local description of the space-time geometry. To discuss the global structure of the whole space it is useful to perform several coordinate transformations. Firstly let us parametrize by $\kappa^{2}=\lambda /(1+\lambda)$ the deformation parameter (with $\kappa<1$ for $\lambda>0$ and $\kappa>1$ for $\lambda<-1$ ) and introduce a radial coordinate $a$ la Horne and Horowitz:

$$
r=\lambda+\cos ^{2} t
$$

which obviously varies between $\lambda$ and $\lambda+1$. The expression of the metric (4.87) becomes in terms of this new coordinate:

$$
\begin{aligned}
\mathrm{d} s^{2} & =-\left[\left(2 \mathrm{H}^{2}(1+\lambda)^{2}-\lambda\right)+\frac{\lambda\left(\lambda-4 \mathrm{H}^{2}(1+\lambda)^{2}\right)}{r}+\frac{\left.\left.2 \lambda^{2} \mathrm{H}^{2}(1+\lambda)^{2}\right)\right)}{r^{2}}\right] \mathrm{d} \psi^{2}+ \\
-(1+\lambda)\left[2 \mathrm{H}^{2}(1+\lambda)+1-\frac{(1+\lambda)\left(1+4 \mathrm{H}^{2}(1+\lambda)^{2}\right)}{r}+\frac{2(1+\lambda)^{3} \mathrm{H}^{2}}{r^{2}}\right] \mathrm{d} \varphi^{2}+ & \\
& +4 \mathrm{H}^{2}(1+\lambda)^{2}\left[1-\frac{1+2 \lambda}{r}+\frac{\lambda(1+\lambda)}{r^{2}}\right] \mathrm{d} \psi \mathrm{d} \varphi+\frac{1}{4(r-\lambda)(r-\lambda-1)} \mathrm{d} r^{2} .
\end{aligned}
$$

This expression looks close to the one discussed by Horne and Horowitz. It also represents a black string. However, it depends on more physical parameters as the expression of the scalar curvature shows:

$$
\mathcal{R}=2 \frac{2 r(1+2 \lambda)-7 \lambda(1+\lambda)-2 \mathrm{H}^{2}(1+\lambda)^{2}}{r^{2}} .
$$


Obviously this metric can be extended behind the initial domain of definition of the $r$ variable. But before to discuss it, it is interesting to note that the Killing vector $\mathbf{k}=(1+\lambda) \partial_{\psi}+\lambda \partial_{\phi} \propto R_{2}$ is of constant square length

$$
\mathbf{k} . \mathbf{k}=\lambda(1+\lambda)-2 \mathrm{H}^{2}(1+\lambda)^{2}:=\omega .
$$

Note that as $\mathrm{H}^{2}$ is positive, we have the inequality $\omega<\lambda(1+\lambda)$. Moreover, in order to have a Lorentzian signature we must impose $\omega>0$. The fact that the Killing vector $\mathbf{k}$ is space-like and of constant length makes it a candidate to perform identifications. We shall discuss this point at the end of this section.

The constancy of the length of the Killing vector $\mathbf{k}$ suggests to make a new coordinate transformation (such that $\mathbf{k}=\partial_{x}$ ) :

$$
\begin{aligned}
& \psi=(1+\lambda) x+t \\
& \varphi=t+\lambda x
\end{aligned}
$$

which leads to the much simpler expression of the line element:

$\mathrm{d} s^{2}=-\frac{(r-\lambda)(r-\lambda-1)}{r^{2}} \mathrm{~d} t^{2}+\omega\left(\mathrm{d} x+\frac{1}{r} \mathrm{~d} t\right)^{2}+\frac{1}{4(r-\lambda)(r-\lambda-1)} \mathrm{d} r^{2}$.

This metric is singular at $r=0, \lambda, \lambda+1 ; r=0$ being a curvature singularity. On the other hand, the volume form is $\sqrt{\omega} /(2 r) \mathrm{d} t \wedge \mathrm{d} x \wedge \mathrm{d} r$, which indicates that the singularities at $r=\lambda$ and $r=\lambda+1$ may be merely coordinate singularities, corresponding to horizons. Indeed, it is the case. If we expand the metric, around $r=\lambda+1$, for instance, at first order (i.e. for $r=\lambda+1+\epsilon$ ) we obtain:

$$
\begin{array}{r}
\mathrm{d} s^{2}=\frac{\omega}{(1+\lambda)^{2}}(\mathrm{~d} t+(1+\lambda) \mathrm{d} x)^{2}-\frac{\epsilon}{(1+\lambda)^{2}} \mathrm{~d} t\left[\mathrm{~d} t+2 \frac{\omega}{1+\lambda}(\mathrm{d} t+(1+\lambda) \mathrm{d} x)\right]+ \\
+\frac{1}{4 \epsilon} \mathrm{d} r^{2}
\end{array}
$$

indicating the presence of a horizon. To eliminate the singularity in the metric, we may introduce Eddington-Finkelstein like coordinates:

$$
\begin{aligned}
& t=(1+\lambda)\left(u \pm \frac{1}{2} \ln \epsilon\right)-\omega \xi \\
& x=\left(1+\frac{\omega}{1+\lambda}\right) \xi-\left(u \pm \frac{1}{2} \ln \epsilon\right) .
\end{aligned}
$$

The same analysis can also be done near the horizon located at $r=\lambda$. Writing $r=\lambda+\epsilon$, the corresponding regulating coordinate transformation to use is given by:

$$
\begin{aligned}
& t=\lambda\left(u \pm \frac{1}{2} \ln \epsilon\right)+\omega \xi \\
& x=\left(1-\frac{\omega}{\lambda}\right) \xi-\left(u \pm \frac{1}{2} \ln \epsilon\right) .
\end{aligned}
$$


In order to reach the null Eddington-Finkelstein coordinates, we must use null rays. The geodesic equations read, in terms of a function $\Sigma^{2}[E, P, \varepsilon ; r]=$ $(E r-P)^{2}-\left(P^{2} / \omega\right)-\varepsilon(r-\lambda)(r-\lambda-1)$ :

$$
\begin{aligned}
\sigma & =\int \frac{1}{4 \Sigma[E, P, \varepsilon ; r]} \mathrm{d} r \\
t & =\int \frac{(E r-P) r}{2(r-\lambda)(r-\lambda-1) \Sigma[E, P, \varepsilon ; r]} \mathrm{d} r \\
x & =-\int \frac{(E r-P)+P / \omega}{2(r-\lambda)(r-\lambda-1) \Sigma[E, P, \varepsilon ; r]} \mathrm{d} r
\end{aligned}
$$

where $E$ and $P$ are the constant of motion associated to $\partial_{t}$ and $\partial_{x}, \sigma$ is an affine parameter and $\varepsilon$, equal to $1,0,-1$, characterizes the time-like, null or space-like nature of the geodesic. Comparing these equations (with $\varepsilon=0$ and $P=0$ ) with the coordinates introduced near the horizons, we see that regular coordinates in their neighbourhoods are given by

$$
\begin{aligned}
& t=T \pm \frac{1}{2}((1+\lambda) \ln |r-\lambda-1|-\lambda \ln |r-\lambda|), \\
& x=X \mp \frac{1}{2}(\ln |r-\lambda-1|-\ln |r-\lambda|),
\end{aligned}
$$

which leads to the metric

$\mathrm{d} s^{2}=\left(-1+\frac{1+2 \lambda}{r}-\frac{\lambda(1+\lambda)-\omega}{r^{2}}\right) \mathrm{d} T^{2}+2 \frac{\omega}{r} \mathrm{~d} X \mathrm{~d} T+\omega \mathrm{d} X^{2} \mp \frac{1}{r} \mathrm{~d} T \mathrm{~d} r$

According to the sign, we obtain incoming or outgoing null coordinates; to build a Kruskal coordinate system we have still to exponentiate them.

Obviously, we may choose the $X$ coordinate in the metric (4.99) to be periodic without introducing closed causal curves. The question of performing more general identifications in these spaces will be addressed below.

We end this section by computing the conserved charges associated to the asymptotic symmetries of our field configurations. As is well known, their expressions provide solutions of the equations of motion derived from the low energy effective action

$$
S=\int d^{d} x \sqrt{-g} \mathrm{e}^{-2 \Phi}\left[R+4(\nabla \Phi)^{2}-\frac{1}{12} H^{2}-\frac{k_{g}}{8} F^{2}+\frac{\delta c}{3}\right],
$$

in which we have choosen the units such that $\delta c=12$.

The expression (4.93) for the metric is particularly appropriate to describe the asymptotic properties of the solution. In these coordinates, the various, non gravitational, fields read as

$$
\begin{aligned}
& F= \pm \frac{\sqrt{2} \mathrm{H}(1+\lambda)}{r^{2} \sqrt{k_{g}}} \mathrm{~d} t \wedge \mathrm{d} r, \\
& H=\mp \frac{\omega}{r^{2}} \mathrm{~d} t \wedge \mathrm{d} x \wedge \mathrm{d} r, \\
& \Phi=\Phi_{\star}-\frac{1}{2} \ln r,
\end{aligned}
$$


By setting $\sqrt{\omega} x=\bar{x}$ and $r=\mathrm{e}^{2 \bar{\rho}}$, near infinity $(\bar{\rho} \rightarrow \infty)$, the metric asymptotes the standard flat metric: $d s^{2}=-d t^{2}+d \bar{x}^{2}+d \bar{\rho}^{2}$, while the fields $F$ and $H$ vanish and the dilaton reads $\Phi=\Phi_{\star}-\bar{\rho}$. This allows to interpret the asymptotic behavior of our solution (4.87) as a perturbation around the solution given by $F=0, H=0$, the flat metric and a linear dilaton: $\bar{\Phi}=\Phi_{\star}+f_{\alpha} X^{\alpha}$, (with here $\left.f_{\alpha}=(0,0,-1)\right)$. Accordingly, we may define asymptotic charges associated to each asymptotic reductibility parameter (see [BB02]).

For the gauge symmetries we obtain as charges, associated to the $H$ field

$$
Q_{H}= \pm 2 \mathrm{e}^{-2 \Phi_{\star}} \sqrt{\omega}
$$

and to the $F$ field

$$
Q_{F}= \pm \frac{2 \sqrt{2} \mathrm{e}^{-2 \Phi_{\star}} \mathrm{H}(1+\lambda)}{\sqrt{k_{g}}} .
$$

The first one reduces (up to normalization) for $\mathrm{H}=0$ to the result given in [HH92], while the second one provides an interpretation of the deformation parameter $\mathrm{H}$.

Moreover, all the Killing vectors of the flat metric defining isometries preserving the dilaton field allow to define asymptotic charges. These charges are obtained by integrating on the surface at infinity the antisymmetric tensor:

$$
k_{\tilde{\xi}}^{[\mu v]}=\mathrm{e}^{-2 \bar{\Phi}}\left(\xi_{\sigma} \partial_{\lambda} \mathcal{H}^{\sigma \lambda \mu \nu}+\frac{1}{2} \partial_{\lambda} \xi_{\sigma} \mathcal{H}^{\sigma \lambda \mu \nu}+2\left(\xi^{\mu} h_{\lambda}^{v} f^{\lambda}-\xi^{v} h_{\lambda}^{\mu} f^{\lambda}\right)\right)
$$

where

$$
\mathcal{H}^{\sigma \lambda \mu v}=\bar{h}^{\sigma v} \eta^{\lambda \mu}+\bar{h}^{\lambda \mu} \eta^{\sigma v}-\bar{h}^{\sigma \mu} \eta^{\lambda v}-\bar{h}^{\lambda v} \eta^{\sigma \mu}
$$

is the well known tensor sharing the symmetries of the Riemann tensor and $\bar{h}^{\mu v}=h^{\mu v}-\frac{1}{2} \eta^{\mu v} \eta^{\alpha \beta} h_{\alpha \beta}$, while the Killing vector $\xi$ has to verify the invariance condition $\xi_{\alpha} f^{\alpha}=0$. The expression of the tensor $k_{\tilde{\xi}}^{[\mu v]}$ depends only on the perturbation $h_{\mu v}$ of the metric tensor because, on the one hand, the $F$ and $H$ fields appear quadratically in the lagrangian, and their background values are zero, while, on the other hand, the perturbation field for the dilaton vanishes: $\Phi=\bar{\Phi}$.

Restricting ourselves to constant Killing vectors, we obtain the momenta (defined for the indice $\sigma=t$ and $\bar{x}$ )

$$
P^{\sigma}=\int \mathrm{d} \bar{x} \mathrm{e}^{-2 \bar{\Phi}}\left(\partial_{\lambda} \mathcal{H}^{\sigma \lambda t \bar{\rho}}-2 \eta^{\sigma t} h_{\bar{\rho}}^{v}\right)
$$

i.e. the density of mass $(\mu)$ and momentum $(\omega)$ per unit length:

$$
\mu=2 \mathrm{e}^{-2 \Phi_{\star}}(1+2 \lambda) \text { and } \quad \omega=-2 \mathrm{e}^{-2 \Phi_{\star}} \sqrt{\omega} .
$$

Of course, if we perform identifications such that the string acquires a finite length, the momenta (4.106) become also finite.

To make an end let us notice that the expressions of $\mu$ and $\omega$ that we obtain differ from those given in [HH92] by a normalization factor but also in their dependance with respect to $\lambda$, even in the limit $\mathrm{H}=0$; indeed, the asymptotic Minkowskian frames used differ from each other by a boost. 
Applications

\section{Discrete identifications}

In the same spirit as the original BTZ construction reminded in the previous section, we would like to investigate to what extent discrete identifications could be performed in the deformed background. Necessary conditions for a solution (4.99) to remain "viable" black hole can be stated as follows:

- the identifications are to be performed along the orbits of some Killing vector $\xi$ of the deformed metric

- there must be causally safe asymptotic regions (at spatial infinity)

- the norm of $\xi$ has to be positive in some region of space-time, and chronological pathologies have to be hidden with respect to an asymptotic safe region by a horizon.

The resulting quotient space will exhibit a black hole structure if, once the regions where $\|\xi\|<0$ have been removed, we are left with an almost geodesically complete space, the only incomplete geodesics being those ending on the locus $\|\xi\|=0$. It is nevertheless worth emphasizing an important difference with the BTZ construction. In our situation, unlike the undeformed $\mathrm{AdS}_{3}$ space, the initial space-time where we are to perform identifications do exhibit curvature singularities.

\section{The BTZ black hole}

In the presence of isometries, discrete identifications provide alternatives for creating new backgrounds. Those have the same local geometry, but differ with respect to their global properties. Whether these identifications can be implemented as orbifolds at the level of the underlying two-dimensional string model is very much dependent on each specific case.

For $\mathrm{AdS}_{3}$, the most celebrated geometry obtained by discrete identification is certainly the BTZ black hole [BTZ92]. The discrete identifications are made along the integral lines of the following Killing vectors (see Eqs. (B.15)):

$$
\begin{aligned}
\text { non-extremal case : } \quad \xi=\left(r_{+}+r_{-}\right) R_{2}-\left(r_{+}-r_{-}\right) L_{2}, \\
\text { extremal case : } \xi=2 r_{+} R_{2}-\left(R_{1}-R_{3}\right)-\left(L_{1}+L_{3}\right) .
\end{aligned}
$$

In the original BTZ coordinates, the metric reads:

$$
\mathrm{d} s^{2}=L^{2}\left[-f^{2}(r) \mathrm{d} t^{2}+f^{-2}(r) \mathrm{d} r^{2}+r^{2}\left(\mathrm{~d} \varphi-\frac{r_{+} r_{-}}{r^{2}} \mathrm{~d} t\right)^{2}\right]
$$

with

$$
f(r)=\frac{1}{r} \sqrt{\left(r^{2}-r_{+}^{2}\right)\left(r^{2}-r_{-}^{2}\right)} .
$$

In this coordinate system,

$$
\partial_{\varphi} \equiv \xi, \partial_{t} \equiv-\left(r_{+}+r_{-}\right) R_{2}-\left(r_{+}-r_{-}\right) L_{2} \text { and } r^{2} \equiv\|\xi\| \text {. }
$$


In $\mathrm{AdS}_{3} \varphi$ is not a compact coordinate. The discrete identification makes $\varphi$ an angular variable, $\varphi \cong \varphi+2 \pi$, which imposes to remove the region with $r^{2}<0$. The BTZ geometry describes a three-dimensional black hole, with mass $M$ and angular momentum $J$, in a space-time that is locally (and asymptotically) antide Sitter. The chronological singularity at $r=0$ is hidden behind an inner horizon at $r=r_{-}$, and an outer horizon at $r=r_{+}$. Between these two horizons, $r$ is time-like. The coordinate $t$ becomes space-like inside the ergosphere, when $r^{2}<r_{\text {erg }}^{2} \equiv r_{+}^{2}+r_{-}^{2}$. The relation between $M, J$ and $r_{ \pm}$is as follows:

$$
r_{ \pm}^{2}=\frac{M L}{2}\left[1 \pm \sqrt{1-\left(\frac{J}{M L}\right)^{2}}\right]
$$

Extremal black holes have $|J|=M L\left(r_{+}=r_{-}\right)$. In the special case $J=M L=0$ one finds the near-horizon geometry of the five-dimensional NS5/F1 stringy black hole in its ground state. Global $\mathrm{AdS}_{3}$ is obtained for $J=0$ and $M L=-1$.

Many subtleties arise, which concern $e . g$. the appearance of closed timelike curves in the excised region of negative $r^{2}$ (where $\partial_{\varphi}$ would have been time-like) or the geodesic completion of the manifold; a comprehensive analysis of these issues can be found in [BHTZ93]. At the string-theory level, the BTZ identification is realized as an orbifold projection, which amounts to keeping invariant states and adding twisted sectors [NS98, HKV02].

Besides the BTZ solution, other locally $\mathrm{AdS}_{3}$ geometries are obtained, by imposing identification under purely left (or right) isometries, refereed to as self-dual (or anti-self-dual) metrics. These were studied in [CH94]. Their classification and isometries are exactly those of the asymmetric deformations studied in the present chapter. The Killing vector used for the identification is (A) time-like (elliptic), (B) space-like (hyperbolic) or (C) null (parabolic), and the isometry group is $U(1) \times S L(2, \mathbb{R})$. It was pointed out in [CH94] that the resulting geometry was free of closed time-like curves only in the case (B).

\section{Discrete identifications in asymmetric deformations}

Our analysis of the residual isometries in purely asymmetric deformations (Sec. 3.1) shows that the vector $\xi$ (Eq. (4.108a)) survives only in the hyperbolic deformation, whereas $\xi$ in Eq. (4.108b) is present in the parabolic one. Put differently, non-extremal BTz black holes allow for electric deformation, while in the extremal ones, the deformation can only be induced by an electro-magnetic wave. Elliptic deformation is not compatible with BTZ identifications.

The question that we would like to address is the following: how much of the original black hole structure survives the deformation? The answer is simple: a new chronological singularity appears in the asymptotic region of the black hole. Evaluating the norm of the Killing vector shows that a naked singularity appears. Thus the deformed black hole is no longer a viable gravitational background. Actually, whatever the Killing vector we consider to perform the identifications, we are always confronted to such pathologies. 
Applications

The fate of the asymmetric parabolic deformation of $\mathrm{AdS}_{3}$ is similar: there is no region at infinity free of closed time-like curves after performing the identifications.

\section{Discrete identifications in symmetric deformations}

Let us consider the symmetric hyperbolic deformation, whose metric is given by (4.93) with $\mathrm{H}=0$, i.e. $\omega=\lambda(1+\lambda)$. This metric has two residual Killing vectors, manifestly given by $\partial_{t}$ and $\partial_{x}$. We may thus, in general, consider identifications along integral lines of

$$
\xi=a \partial_{t}+\partial_{x}
$$

This vector has squared norm:

$$
\|\xi\|^{2}=\left(\lambda(1+\lambda)-a^{2}\right)+\frac{a \lambda(1+\lambda)+a^{2}(1+2 \lambda)}{r} .
$$

To be space-like at infinity the vector $\xi$ must verify the inequality $\lambda(1+\lambda)>$ $a^{2}$. If $a>0$, or $-\sqrt{\lambda(1+\lambda)}<a<-2 \lambda(1+\lambda) /(1+2 \lambda), \xi$ is everywhere space-like. Otherwise, it becomes time-like behind the inner horizon $(r=\lambda)$, or on this horizon if $a=-\lambda$. In this last situation, the quotient space will exhibit a structure similar to that of the black string, with a time-like singularity (becoming light-like for $a=-\lambda$ ) and two horizons.

\section{Discrete identifications in double deformations}

The norm squared of the identification vector (4.113) in the metric (4.93) is

$$
\|\xi\|^{2}=\left(\omega-a^{2}\right)+2 \frac{a \omega+a^{2}(1+2 \lambda)}{r}-\frac{a^{2}(\lambda(1+\lambda)-\omega)}{r^{2}} .
$$

Between $r=0$ and $r=\infty$, this scalar product vanishes once and only once (if $a \neq 0$ ). To be space-like at infinity we have to restrict the time component of $\xi$ to $|a|<\omega$. Near $r=0$ it is negative, at the outer horizon $(r=\lambda+1)$ it takes the positive value $\omega(1+\lambda+a)^{2} /(1+\lambda)^{2}$ and near the inner horizon $(r=\lambda)$ the non-negative value $\omega(\lambda+a)^{2} / \lambda^{2}$. Accordingly, by performing identifications using this Killing vector, we will encounter a chronological singularity, located at $r=r^{*}$, with $0<r^{*}<\lambda+1$. When $r^{*}<\lambda$, the singularity will be of the same type as the one in the symmetric case. But when $\lambda<r^{*}<\lambda+1$, the chronological singularity will be space-like, and the causal structure we get is much like that of the Schwarschild black hole, as is shown in Fig. 4.1.

\section{Towards the exact spectra}

Let us consider the algebraic point of view. Again as in the electric deformation of $S L(2, \mathbb{R})$ we can't write the partition function but we must content ourselves with the spectrum which will generalize what we found in Eqs. (4.33a) and (4.33b). 


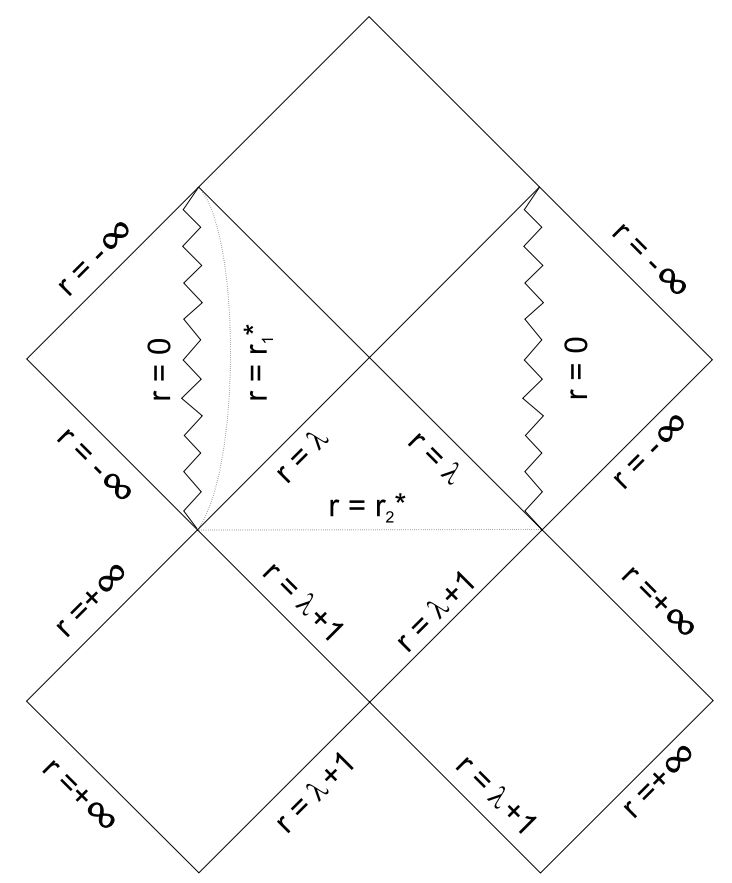

Figure 4.1: Penrose diagram exhibiting the global structure of the double hyperbolic deformation. The time-like curvature singularities $r=0$ are represented, as well as the horizons, located at $r=\lambda$ and $r=\lambda+1$. When performing identifications along orbits of a Killing vector allowing for a causally safe region at infinity, there appears chronological singularities, which can be timelike and hidden behind an outer and an inner horizon $\left(r=r_{1}^{*}\right)$, or space-like and hidden behind a single horizon $\left(r=r_{2}^{*}\right)$, while the regions where $r<r^{*}$ have to be removed.

\section{Deformed Spectrum}

Consider the double deformation described above for a $S L(2, \mathbb{R})_{k}$ super-WZW model where $J$ is the hyperbolic (space-like) $J_{2}$ current.

The evaluation of the spectrum for our deformed model is pretty straightforward once one realizes that the deformations act as $O(2,2)$ pseudo-orthogonal transformations on the charge lattice corresponding to the abelian subgroup of the $\mathfrak{s l}(2, \mathbb{R})$ heterotic model (as described in Sec. 3.1). Left and right weights for the relevant lattices are (see Eqs. (D.20) and (D.21)):

$$
\begin{aligned}
& L_{0}=\frac{1}{k}\left(\mu+n+\frac{a}{2}\right)^{2}, \\
& \bar{L}_{0}=\frac{\bar{\mu}^{2}}{k+2}+\frac{1}{k_{g}}\left(\bar{n}+\frac{\bar{a}}{2}\right)^{2},
\end{aligned}
$$

where the anti-holomorphic part contains the contribution coming from a $\mathfrak{u}(1)$ subgroup of the heterotic gauge group.

At the Lagrangian level, the infinitesimal deformation we want to describe 
is given by the following marginal operator:

$$
\mathcal{O}=\kappa^{2} \frac{\left(J^{2}+\imath \psi_{1} \psi_{3}\right)}{\sqrt{k}} \frac{\bar{J}^{2}}{\sqrt{k+2}}+\mathrm{H} \frac{\left(J^{2}+\imath \psi_{1} \psi_{3}\right)}{\sqrt{k}} \frac{\bar{I}}{\sqrt{k_{g}}} .
$$

This suggests that the actual $O(2,2)$ transformation should be obtained as a boost between the holomorphic part and the result of a rotation between the two anti-holomorphic components. The deformed lattices then read:

$$
\begin{aligned}
& L_{0}^{\mathrm{dd}}=\left\{\frac{1}{\sqrt{k}}\left(\mu+n+\frac{a}{2}\right) \cosh x+\left(\frac{\bar{\mu}}{\sqrt{k+2}} \cos \alpha+\frac{1}{\sqrt{k_{g}}}\left(\bar{n}+\frac{\bar{a}}{2}\right) \sin \alpha\right) \sinh x\right\}^{2}, \\
& \bar{L}_{0}^{\mathrm{dd}}=\left\{\left(\frac{\bar{\mu}}{\sqrt{k+2}} \cos \alpha+\frac{1}{\sqrt{k_{g}}}\left(\bar{n}+\frac{\bar{a}}{2}\right) \sin \alpha\right) \cosh x+\frac{1}{\sqrt{k}}\left(\mu+n+\frac{a}{2}\right) \sinh x\right\}^{2},
\end{aligned}
$$

where the parameters $x$ and $\alpha$ can be expressed as functions of $\kappa$ and $\mathrm{H}$ as follows:

$$
\left\{\begin{array}{l}
\kappa^{2}=\sinh (2 x) \cos \alpha \\
\mathrm{H}=\sinh (2 x) \sin \alpha
\end{array}\right.
$$

Of course this is a generalization of the expressions in Eq. (4.33).

\section{Twisting}

The identification operation we performed in the symmetrically and doubledeformed metric (as in Sec. 4.4) is implemented in the string theory framework by the orbifold construction. This was already obtained in [NS98, HKV02] for the "standard" BTZ black hole that was described as a $S L(2, \mathbb{R}) / \mathbb{Z}$ orbifold.

In order to write the spectrum that will contain the twisted sectors, the first step consists in writing explicitly the primary fields in our theory, distinguishing between the holomorphic and anti-holomorphic parts (as it is natural to do since the construction is intrinsically heterotic).

- The holomorphic part is written by introducing the charge boost of Eq. (4.118a) in Eq. (D.16):

$$
\Phi_{j \mu \nu \bar{\mu} \bar{v}}^{\mathrm{dd}}(z)=U_{j \mu}(z) \exp \left[\imath\left(\sqrt{\frac{2}{k}}\left(\mu+n+\frac{a}{2}\right) \cosh x+\sqrt{2} \bar{Q}_{\alpha} \sinh x\right) \vartheta_{2}\right],
$$

where $Q_{\alpha}=\bar{\mu} \sqrt{\frac{2}{k+2}} \cos \alpha+\bar{v} \sqrt{\frac{2}{k_{g}}} \sin \alpha$ and the dd superscript stands for double deformed 
- To write the anti-holomorphic part we need at first to implement the rotation between the $\bar{J}^{3}$ and gauge current components:

$$
\begin{aligned}
\bar{\Phi}_{j \bar{\mu} \bar{v}}(\bar{z}) & =V_{j \mu}(\bar{z}) e^{\imath \bar{\mu} \sqrt{2 / k+2} \bar{\theta}_{2}} e^{\imath \bar{v} \sqrt{2 / k_{g}} \bar{X}}= \\
= & V_{j \mu}(\bar{z}) e^{\imath \sqrt{2} \bar{Q}_{\alpha}\left(\bar{\theta}_{2} \cos \alpha+\bar{X} \sin \alpha\right)} e^{\imath \sqrt{2} \bar{Q}_{\alpha-\pi / 2}\left(-\bar{\theta}_{2} \sin \alpha+\bar{X} \cos \alpha\right)},
\end{aligned}
$$

and then realize the boost in Eq. (4.118b) on the involved part:

$$
\begin{aligned}
& \bar{\Phi}_{j \mu \bar{\mu} \nu \bar{v}}^{\mathrm{dd}}(\bar{z})=V_{j \mu} e^{\imath \sqrt{2} \bar{Q}_{\alpha-\pi / 2}\left(-\bar{\theta}_{2} \sin \alpha+\bar{X} \cos \alpha\right)} \times \\
& \times \exp \left[\imath\left(\sqrt{\frac{2}{k}}\left(\mu+n+\frac{a}{2}\right) \sinh x+\sqrt{2} \bar{Q}_{\alpha} \cosh x\right)\left(\bar{\theta}_{2} \cos \alpha+\bar{X} \sin \alpha\right)\right] .
\end{aligned}
$$

Now that we have the primaries, consider the operator $W_{w}(z, \bar{z})$ defined as follows:

$$
W_{w}(z, \bar{z})=e^{-\imath \frac{k}{2} w \Delta_{-} \vartheta_{2}+l \frac{k+2}{2} w \Delta_{+} \bar{\theta}_{2}}
$$

where $w \in \mathbb{Z}$ and $\bar{\theta}_{2}$ the boson corresponding to the $\bar{J}_{2}$ current. It is easy to show that the following OPE's hold:

$$
\begin{aligned}
& \vartheta_{2}(z) W_{n}(0, \bar{z}) \sim-\imath w \Delta_{-} \log z W_{w}(0, \bar{z}), \\
& \bar{\theta}_{2}(\bar{z}) W_{n}(z, 0) \sim \imath w \Delta_{+} \log \bar{z} W_{w}(z, 0),
\end{aligned}
$$

showing that $W_{w}(z, \bar{z})$ acts as twisting operator with winding number $w\left(\vartheta_{2}\right.$ and $\bar{\theta}_{2}$ shift by $2 \pi \Delta_{-} w$ and $2 \pi \Delta_{+} w$ under $\left.z \rightarrow e^{2 \pi \imath} z\right)$. This means that the general primary field in the $S L(2, \mathbb{R})_{k} / \mathbb{Z}$ theory can be written as:

$$
\Phi_{j \mu \bar{\mu} v \bar{v} w}^{\mathrm{tw}}(z, \bar{z})=\Phi_{j \mu \bar{\mu} v \bar{v}}^{\mathrm{dd}}(z, \bar{z}) W_{w}(z, \bar{z}) .
$$

where the tw superscript stands for twisted.

Having the explicit expression for the primary field, it is simple to derive the scaling dimensions which are obtained, as before, via the GKO decomposition of the Virasoro algebra $T[\mathfrak{s l}(2, \mathbb{R})]=T[\mathfrak{s l}(2, \mathbb{R}) / \mathfrak{o}(1,1)]+T[\mathfrak{o}(1,1)]$. Given that the $T[\mathfrak{s l}(2, \mathbb{R}) / \mathfrak{o}(1,1)]$ part remains invariant (and equal to $L_{0}=$ $-j(j+1) / k-\mu^{2} /(k+2)$ as in Eq. (D.18)), the deformed weights read:

$$
\begin{aligned}
& L_{0}^{\mathrm{tw}}=\left\{\frac{k}{2 \sqrt{2}} w \Delta_{-}+\frac{1}{\sqrt{k}}\left(\mu+n+\frac{a}{2}\right) \cosh x+\bar{Q}_{\alpha} \sinh x\right\}^{2} \\
& \bar{L}_{0}^{\mathrm{tw}}=\left\{-\frac{k+2}{2 \sqrt{2}} w \Delta_{+} \cos \alpha+\bar{Q}_{\alpha} \cosh x+\frac{1}{\sqrt{k}}\left(\mu+n+\frac{a}{2}\right) \sinh x\right\}^{2}+ \\
&+\left\{\frac{k+2}{2 \sqrt{2}} w \Delta_{+} \sin \alpha+\bar{Q}_{\alpha-\pi / 2}\right\}^{2}
\end{aligned}
$$


Applications

\subsection{New compactifications}

Up to this point we have focused on the squashed and coset models under the underlying hypothesis that they act as parts of larger ten-dimensional backgrounds. In this section we will study other examples which are likely to be part of physically sound models. In particular we will closely study the $S U(3) / U(1)^{2}$ coset that can be used as the six-dimensional compact counterpart of an $\mathrm{AdS}_{4}$ background.

\section{The $S U(3) / U(1)^{2}$ flag space}

Let us now consider the next example in terms of coset dimensions, $S U(3) / U(1)^{2}$. As a possible application for this construction we may think to associate this manifold to a four-dimensional $(1,0)$ superconformal field theory $\mathcal{M}$ so to compactify a critical string theory since $\operatorname{dim}\left[S U(3) / U(1)^{2}\right]=8-2=6$. Our construction gives rise to a whole family of CFT's depending on two parameters (since rank $[S U(3)]=2$ ) but in this case we are mainly interested to the point of maximal deformation, where the $U(1)^{2}$ torus decouples and we obtain an exact theory on the $S U(3) / U(1)^{2}$ coset. Before giving the explicit expressions for the objects in our construction it is hence useful to recall some properties of this manifold. The first consideration to be made is the fact that $S U(3) / U(1)^{2}$ is an asymmetric coset in the mathematical sense defined in Sec. 3.3 (as we show below). This allows for the existence of more than one left-invariant Riemann metric. In particular, in this case, if we just consider structures with constant Ricci scalar, we find, together with the restriction of the Cartan-Killing metric on $S U$ (3), the Kähler metric of the flag space $F^{3}$. The construction we present in this section will lead to the first one of these two metrics. This is known to admit a nearly-Kähler structure and has already appeared in the superstring literature as a basis for a cone of $G_{2}$ holonomy [AW03].

Gauss A suitable parametrisation for the $S U$ (3) group is obtained via the Gauss decomposition decomposition described in App. B.3. In these terms the general group elefor $\operatorname{SU}(3)$

ment is written as:

$g\left(z_{1}, z_{2}, z_{3}, \psi_{1}, \psi_{2}\right)=\left(\begin{array}{ccc}\frac{e^{\imath \psi_{1} / 2}}{\sqrt{f_{1}}} & -\frac{\bar{z}_{1}+z_{2} \bar{z}_{3}}{\sqrt{f 1 f 2}} e^{\imath\left(\psi_{1}-\psi_{2}\right) / 2} & -\frac{\bar{z}_{3}-\bar{z}_{1} \bar{z}_{2}}{\sqrt{f_{2}}} e^{-\imath \psi_{2} / 2} \\ \frac{z_{1} e^{\imath \psi_{1} / 2}}{\sqrt{f_{1}}} & -\frac{1+\left|z_{3}\right|^{2}-z_{1} z_{2} \bar{z}_{3}}{\sqrt{f 1 f 2}} e^{\imath\left(\psi_{1}-\psi_{2}\right) / 2} & -\frac{\bar{z}_{2}}{\sqrt{f_{2}}} e^{-\imath \psi_{2} / 2} \\ \frac{z_{3} e^{\imath \psi_{1} / 2}}{\sqrt{f_{1}}} & -\frac{z_{2}-\bar{z}_{1} z_{3}+z_{2}\left|z_{1}\right|^{2}}{\sqrt{f 1 f 2}} e^{\imath\left(\psi_{1}-\psi_{2}\right) / 2} & \frac{1}{\sqrt{f_{2}}} e^{-\imath \psi_{2} / 2}\end{array}\right)$

where $z_{i}$ are three complex parameters, $\psi_{j}$ are two real parameters and $f_{1}=$ $1+\left|z_{1}\right|^{2}+\left|z_{3}\right|^{2}, f_{2}=1+\left|z_{2}\right|^{2}+\left|z_{3}-z_{1} z_{2}\right|^{2}$. As for the group, we need also an explicit parametrisation for the $\mathfrak{s u}$ (3) algebra, such as the one provided by the Gell-Mann matrices in Eq. (B.40). It is a well known result that if a Lie algebra is semi-simple (or, equivalently, if its Killing form is negative-definite) 
then all Cartan subalgebras are conjugated by some inner automorphism ${ }^{15}$. This leaves us the possibility of choosing any couple of commuting generators, knowing that the final result won't be influenced by such a choice. In particular, then, we can pick the subalgebra generated by $\mathfrak{k}=\left\langle\lambda_{3}, \lambda_{8}\right\rangle{ }^{16}$

The holomorphic currents of the bosonic $S U(3)_{k}$ corresponding to the two operators in the Cartan are:

$$
\mathcal{J}^{3}=-\left\langle\lambda_{3} g\left(z_{\mu}, \psi_{a}\right)^{-1} \mathrm{~d} g\left(z_{\mu}, \psi_{a}\right)\right\rangle \quad \mathcal{J}^{8}=-\left\langle\lambda_{8} g\left(z_{\mu}, \psi_{a}\right)^{-1} \mathrm{~d} g\left(z_{\mu}, \psi_{a}\right)\right\rangle
$$

and in these coordinates they read:

$$
\begin{gathered}
\mathcal{J}^{3}=-\frac{\imath}{\sqrt{2}}\left\{\left(\frac{z_{1}}{f_{1}}+\frac{z_{2}\left(-\bar{z}_{1} \bar{z}_{2}+\bar{z}_{3}\right)}{2 f_{2}}\right) \mathrm{d} z_{1}-\frac{\bar{z}_{2}\left(1+\left|z_{1}\right|^{2}\right)-z_{1} \bar{z}_{3}}{2 f_{2}} \mathrm{~d} z_{2}+\left(\frac{\bar{z}_{3}}{f_{1}}+\frac{\bar{z}_{1} \bar{z}_{2}-\bar{z}_{3}}{2 f_{2}}\right) \mathrm{d} z_{3}\right\} \\
+ \text { c.c. }+\frac{\mathrm{d} \psi_{1}}{\sqrt{2}}-\frac{\mathrm{d} \psi_{2}}{2 \sqrt{2}} \\
\mathcal{J}^{8}=-1 \sqrt{\frac{3}{2}}\left\{\frac{\bar{z}_{1} \bar{z}_{2}-\bar{z}_{3}}{2 f_{2}} z_{2} \mathrm{~d} z_{1}+\frac{\bar{z}_{2}+\left|z_{1}\right|^{2} \bar{z}_{2}-z_{1} \bar{z}_{3}}{2 f_{2}} \mathrm{~d} z_{2}+\frac{-\bar{z}_{1} \bar{z}_{2}+\bar{z}_{3}}{2 f_{2}} \mathrm{~d} z_{3}\right\}+\text { c.c. }+\frac{1}{2} \sqrt{\frac{3}{2}} \mathrm{~d} \psi_{2} .
\end{gathered}
$$

Those currents appear in the expression of the exactly marginal operator that we can add to the SU (3) WZW model action:

$$
\begin{aligned}
V=\frac{\sqrt{k k_{g}}}{2 \pi} \mathrm{H} \int \mathrm{d} z^{2} \mathrm{H}_{3} & \left(J^{3}-\frac{\imath}{\sqrt{2} k}\left(2: \psi_{2} \psi_{1}:+: \psi_{5} \psi_{4}:+: \psi_{7} \psi_{6}:\right)\right) \bar{J}^{3}+ \\
& +\mathrm{H}_{8}\left(J^{8}-\frac{\imath}{k} \sqrt{\frac{3}{2}}\left(: \psi_{5} \psi_{4}:+: \psi_{7} \psi_{6}:\right)\right) \bar{J}^{8}
\end{aligned}
$$

where $\psi^{i}$ are the bosonic current superpartners and $\bar{J}^{3}, \bar{J}^{8}$ are two currents from the gauge sector both generating a $U(1)_{k_{g}}$.

Since rank $[S U(3)]=2$ we have a bidimensional family of deformations parametrised by the two moduli $\mathrm{H}_{3}$ and $\mathrm{H}_{8}$. The back-reaction on the metric is given by:

$$
\mathrm{d} s^{2}=g_{\alpha \bar{\beta}} \mathrm{d} z^{\alpha} \otimes \mathrm{d} \bar{z}^{\beta}+\left(1-2 \mathrm{H}_{3}^{2}\right) \mathcal{J}^{3} \otimes \mathcal{J}^{3}+\left(1-2 \mathrm{H}_{8}^{2}\right) \mathcal{J}^{8} \otimes \mathcal{J}^{8}
$$

where $g_{\alpha \bar{\beta}}$ is the restriction of the $S U$ (3) metric on $S U(3) / U(1)^{2}$. It is worth to remark that for any value of the deformation parameters $\mathrm{H}_{3}$ and $\mathrm{H}_{8}$ the deformed metric is Einstein with constant Ricci scalar.

\footnotetext{
${ }^{15}$ This is the reason why the study of non-semi-simple Lie algebra deformation constitutes a richer subject. In example the $S L(2, \mathbb{R})$ group admits for 3 different deformations, leading to 3 different families of exact CFT's with different physics properties. On the other hand the 3 possible deformations in $S U$ (3) are equivalent.

${ }^{16}$ In this explicit parametrisation it is straightforward to show that the coset we're considering is not symmetric. It suffices to pick two generators, say $\lambda_{2}$ and $\lambda_{4}$, and remark that their commutator $\left[\lambda_{2}, \lambda_{4}\right]=-1 / \sqrt{2} \lambda_{6}$ doesn't live in the Cartan subalgebra.
} 
Applications

With a procedure that has by now become familiar we introduce the following reparametrization:

$$
\psi_{1}=\frac{\hat{\psi}_{1}}{\sqrt{1-2 \mathrm{H}^{2}}} \quad \psi_{2}=\frac{\hat{\psi}_{2}}{\sqrt{1-2 \mathrm{H}^{2}}}
$$

and take the $\mathrm{H}_{3} \rightarrow 1 / \sqrt{2}, \mathrm{H}_{8} \rightarrow 1 / \sqrt{2}$ limit. The resulting metric is:

$$
\mathrm{d} s^{2}=g_{\alpha \bar{\beta}} \mathrm{d} z^{\alpha} \otimes \mathrm{d} \bar{z}^{\beta}+\frac{\mathrm{d} \hat{\psi}_{1} \otimes \mathrm{d} \hat{\psi}_{1}-\mathrm{d} \hat{\psi}_{1} \otimes \mathrm{d} \hat{\psi}_{2}+\mathrm{d} \hat{\psi}_{2} \otimes \mathrm{d} \hat{\psi}_{2}}{2}
$$

that is the metric of the tangent space to the manifold $S U(3) / U(1)^{2} \times U(1) \times$ $U(1)$. The coset metric hence obtained has a $\mathbb{C}$-structure, is Einstein and has constant Ricci scalar $R=15 / k$. The other background fields at the boundary of the moduli space read:

$F=\mathrm{d} \mathcal{J}^{3}+\mathrm{d} \mathcal{J}^{8}$

$H_{[3]}=-3 \sqrt{2}\left\{\mathcal{J}^{1} \wedge\left(\mathcal{J}^{4} \wedge \mathcal{J}^{5}-\mathcal{J}^{6} \wedge \mathcal{J}^{7}\right)+\sqrt{3} \mathcal{J}^{2} \wedge\left(\mathcal{J}^{4} \wedge \mathcal{J}^{5}+\mathcal{J}^{6} \wedge \mathcal{J}^{7}\right)\right\}$

Superymmetry properties of $S U(3) / U(1)^{2}$
If we consider the supersymmetry properties along the deformation line we can remark the presence of an interesting phenomenon. The initial SU (3) model has $N=2$ but this symmetry is naively broken to $N=1$ by the deformation. This is true for any value of the deformation parameter but for the boundary point $\mathrm{H}_{3}^{2}=\mathrm{H}_{8}^{2}=1 / 2$ where the $\mathrm{N}=2$ supersymmetry is restored. Following [GHR84, KS89b, KS89a] one can see that a G/T coset admits $N=2$ supersymmetry if it possesses a complex structure and the corresponding algebra can be decomposed as $\mathfrak{j}=\mathfrak{j}_{+} \oplus \mathfrak{j}_{-}$such as $\left[\mathfrak{j}_{+}, \mathfrak{j}_{+}\right]=\mathfrak{j}_{+}$and $\left[\mathfrak{j}_{-}, \mathfrak{j}_{-}\right]=\mathfrak{j}_{-}$. Explicitly, this latter condition is equivalent (in complex notation) to $f_{i j k}=f_{i \bar{j} k}=f_{a i j}=f_{a \bar{i}}=0$. These are easily satisfied by the $S U(3) / U(1)^{2}$ coset (and actually by any $G / T$ coset) since the commutator of two positive (negative) roots can only be proportional to the positive (negative) root obtained as the sum of the two or vanish.. Having $N=2$ supersymmetry is equivalent to asking for the presence of two complex structures. The first one is trivially given by considering positive roots as holomorphic and negative roots as anti-holomorphic, the other one by interchanging the role in one out of the three positive/negative couples (the same flip on two couples would give again the same structure and on all the three just takes back to the first structure). The metric is Hermitian with respect to both structures since it is $S U$ (3) invariant. It is worth to remark that such background is different from the ones described in [KS89a] because it is not Kähler and can't be decomposed in terms of Hermitian symmetric spaces.

\section{Different constructions on $S U(3) / U(1)^{2}$}

To study the SU (3) case we will use the "current" approach of Sec. 3.6, since a direct computation in coordinates would be impractical. As one could expect, the study of $S U$ (3) deformation is quite richer because of the presence of an 
embedded SU (2) group that can be gauged. Basically this means that we can choose two different deformation patterns that will lead to the two possible Einstein structures that can be defined on the $S U(3) / U(1)^{2}$ manifold.

\section{Direct gauging.}

The first possible choice leads to the same model as before by simply gauging the $U(1)^{2}$ Cartan torus. Consider the initial SU $(3)_{k} \times U(1)_{k^{\prime}} \times U(1)_{k^{\prime \prime}}$ model. In the $\left\langle\mathcal{J}_{1}, \ldots, \mathcal{J}_{8}, \mathcal{I}_{1}, \mathcal{I}_{2}\right\rangle$ base $\left(\left\{\mathcal{J}_{i}\right\}\right.$ being the $S U$ (3) generators and $\left\{\mathcal{I}_{k}\right\}$ the $2 U(1)$ 's), the initial metric is written as:

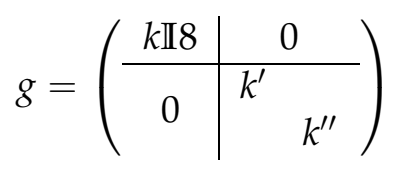

the natural choice for the Cartan torus is given by the usual $\left\langle\mathcal{J}_{3}, \mathcal{J}_{8}\right\rangle$ generators, so we can proceed as before and write the deformed metric as:

$$
g=\left(\begin{array}{llllll}
k \amalg 2 & & & & \\
& \lambda_{1}\left(k, k^{\prime}, \mathrm{H}_{3}\right) & & & \\
& & k \amalg 4 & & \\
& & & \lambda_{1}\left(k, k^{\prime \prime}, \mathrm{H}_{8}\right) & \\
& & & \lambda_{2}\left(k, k^{\prime}, \mathrm{H}_{3}\right) & \\
& & & & \lambda_{2}\left(k, k^{\prime \prime}, \mathrm{H}_{8}\right)
\end{array}\right)
$$

where $\mathrm{H}_{3}$ and $\mathrm{H}_{8}$ are the deformation parameters and $\lambda_{1}$ and $\lambda_{2}$ are the eigenvalues for the interaction matrices, given in Eq. (3.118). In particular, then, in the $\mathrm{H}_{3}^{2} \rightarrow 1 / 2, \mathrm{H}_{8}^{2} \rightarrow 1 / 2$ limit two eigenvalues vanish, the corresponding directions decouple and we are left with the following (asymmetrically gauged) model:

$$
g=\left(\begin{array}{l|ll}
k \Perp 6 & & \\
\hline & k+k^{\prime} & \\
& & k+k^{\prime \prime}
\end{array}\right)
$$

in the $\left\langle\mathcal{J}_{1}, \mathcal{J}_{2}, \mathcal{J}_{4}, \mathcal{J}_{5}, \mathcal{J}_{6}, \mathcal{J}_{7}, \sqrt{k^{\prime}} \mathcal{I}_{1}+\sqrt{k} \mathcal{J}_{3}, \sqrt{k^{\prime \prime}} \mathcal{I}_{2}+\sqrt{k} \mathcal{J}_{8}\right\rangle$ basis that can be seen as a $U(1)^{2}$ fibration over an $S U(3) / U(1)^{2}$ base with metric diag $(1,1,1,1,1,1)$ (in the current basis). This is precisely the same result we obtained in the previous section when we read the fibration as a gauge field living on the base.

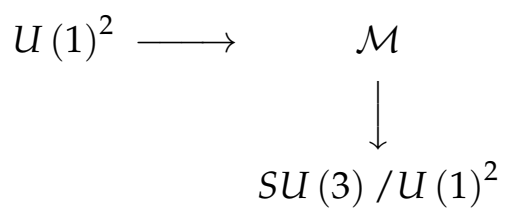

As in the previous example all this construction is valid only if the asymmetrically gauged WZW model is anomaly-free. 
Applications

\section{The $F_{3}$ flag space}

Let us now turn to the other possible choice for the $S U$ (3) gauging, namely the one where we take advantage of the $S U$ (2) embedding. Let us then consider the $S U(3)_{k_{3}} \times S U(2)_{k_{2}} \times U(1)_{k^{\prime}} \times U(1)_{k^{\prime \prime}}$ WZW model whose metric is

$g=\left(\begin{array}{l|l|ll}k_{3} I 8 & & & \\ \hline & k_{2} I 3 & & \\ \hline & & k^{\prime} & \\ & & & k^{\prime \prime}\end{array}\right)$

in the $\left\langle\mathcal{J}_{1}, \ldots, \mathcal{J}_{8}, \mathcal{I}_{1}, \mathcal{I}_{2}, \mathcal{I}_{3}, \mathcal{K}_{1}, \mathcal{K}_{2}\right\rangle$ basis, where $\left\langle\mathcal{J}_{i}\right\rangle$ generate the $S U(3),\left\langle\mathcal{I}_{i}\right\rangle$ generate the $S U(2)$ and $\left\langle\mathcal{K}_{i}\right\rangle$ generate the $U(1)^{2}$.

The first step in this case consists in an asymmetric gauging mixing the $\left\{\mathcal{J}_{1}, \mathcal{J}_{2}, \mathcal{J}_{3}\right\}$ and $\left\{\mathcal{I}_{1}, \mathcal{I}_{2}, \mathcal{I}_{3}\right\}$ currents respectively. At the gauging point, a whole 3-sphere decouples and we obtain the following metric:

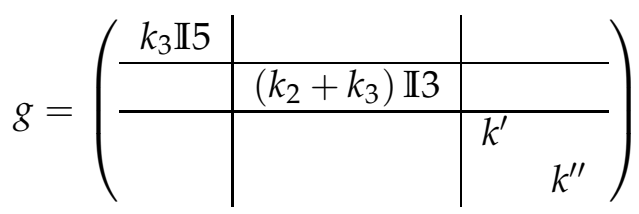

where we have to remember that in order to have an admissible embedding $k_{2}=k_{3}=k$. Our result is again - not surprisingly - a $S U$ (2) fibration over a $S U$ (3) / SU (2) base (times the two U (1)'s).

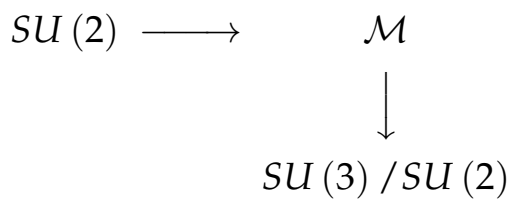

Of course one could be tempted to give $\mathcal{M}$ the same interpretation as before, namely an $S U$ (3) / SU (2) space supported by a chromo-magnetic SU (2) field (or, even better, gauging an additional $U(1)$, of a $\mathbb{C P}^{2}$ background with an $S U(2) \times U(1)$ chromo-magnetic field). Actually this is not the case. The main point is the fact that this $S U(3) \times S U(2)$ model is essentially different from the previous ones because the $U(1)$ factors were the result of the bosonization of the right-moving gauge current which in this way received a (fake) left-moving partner as in Sec. 3.1. This is not possible in the non-abelian case since one can't obtain an SU (2) at arbitrary level $k$ out of the fermions of the theory ${ }^{17}$. In other words, the $S U(2)$ factor is in this case truly a constituent of the theory and there is no reason why it should be decoupled or be given a different interpretation from the $S U$ (3) part. This is why the structure obtained by the $S U(2)$ asymmetric gauging is to be considered an eightdimensional space admitting an $S U(2) \rightarrow S U(3) / S U(2)$ fibration structure,

\footnotetext{
${ }^{17}$ This would be of course be possible if we limited ourselves to small values of $k$, but in this case the whole geometric interpretation of the background would be questionable. However for Gepner-like string compactifications this class of models is relevant.
} 
or, equivalently, a deformed $S U$ (3) where an embedded $S U(2)$ is at a level double with respect to the other generators.

On the other hand we are still free to gauge away the two $U(1)$ factors just as before. This time we can choose to couple $K_{1}$ with the $\mathcal{J}_{8}$ factor that was left untouched in the initial $S U(3)$ and $\mathcal{K}_{2}$ with the $\mathcal{J}_{3}+\mathcal{I}_{3}$ generator. Again we find a two-parameter family of deformations whose metric can be written as:

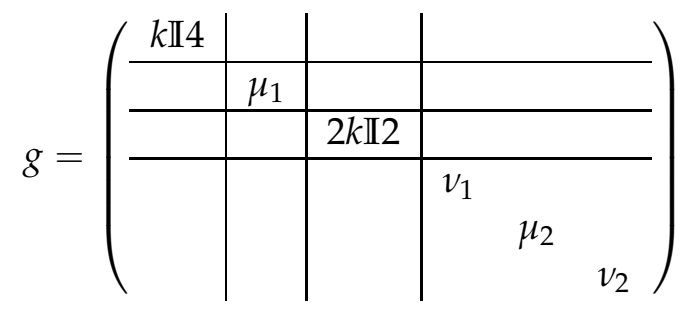

where:

$$
\begin{aligned}
& \mu=\lambda\left(k, k^{\prime}, \mathrm{H}^{\prime}\right) \\
& v=\lambda\left(2 k, k^{\prime \prime}, \mathrm{H}^{\prime \prime}\right) .
\end{aligned}
$$

In particular now we can take the decoupling $\mathrm{H}^{\prime}=\mathrm{H}^{\prime \prime} \rightarrow 1 / 2$ limit where we obtain:

$$
g=\left(\begin{array}{l|l|ll}
k \amalg 4 & & & \\
\hline & 2 k \amalg 2 & & \\
\hline & & k+k^{\prime} & \\
& & & 2 k+k^{\prime \prime}
\end{array}\right)
$$

this structure is once more a $U(1)^{2} \rightarrow S U(3) / U(1)^{2}$ fibration but in this case it is perfectly fine to separate the space components from the gauge field ones. So we can read out our final background fields as the Kähler metric on $F_{3}$ supported by a $U(1)^{2}$ (chromo)magnetic field.

To summarize our results we can say that the two Einstein structures that one can define on $S U(3) / U(1)^{2}$ are both exact string theory backgrounds:

- The first one, obtained as the asymmetric coset $\frac{S U(3) \times U(1)^{2}}{U(1)^{2}}$ is supported by an NS-NS field strength and a magnetic field;

- The second, corresponding to the $\frac{S U(3) \times S U(2) \times U(1)^{2}}{S U(2) \times U(1)^{2}}$ asymmetric coset is Kähler and hence supported by the (chromo-)magnetic field alone.

This Kähler structure has been deeply studied both from the mathematical and physical points of view. In particular the Kähler form can be written as in

Kähler form for $S U(3) / U(1)^{2}$ App. B.3:

$$
K\left(\gamma_{\mu}, \bar{\gamma}_{\mu}\right)=\log \left[1+\left|\gamma_{1}\right|^{2}+\left|\gamma_{3}\right|^{2}\right]+\log \left[1+\left|\gamma_{2}\right|^{2}+\left|\gamma_{3}-\gamma_{1} \gamma_{2}\right|^{2}\right] .
$$

It is immediate to show that this manifold is Einstein and in particular its Ricci scalar is $R=12$. Being Kähler, $F_{3}$ is torsionless, that means in turn that there 
is no NS-NS form ${ }^{18}$. Moreover there is no dilaton by construction ${ }^{19}$. The only other field that supports the background comes from the $U(1)^{2}$ fibration. Since the manifold is Kähler it is useful to take advantage of the complex structure and write our background fields in complex formalism. In these terms the metric is written as:

$$
g=\frac{k}{2}\left(\mathcal{J}^{1} \otimes \mathcal{J}^{\overline{1}}+\mathcal{J}^{2} \otimes \mathcal{J}^{\overline{2}}+2 \mathcal{J}^{3} \otimes \mathcal{J}^{\overline{3}}\right)
$$

where $\mathcal{J}^{i}$ and $\overline{\mathcal{J}}^{i}$ are the Maurer-Cartan corresponding to positive and negative roots respectively and the field strength is given by:

$$
F^{a}=\sqrt{\frac{k}{2 k_{g}}} f_{\mu \bar{\rho}}^{a} C^{\bar{\rho} \sigma} R_{\sigma \bar{v}} \mathcal{J}^{\mu} \wedge \mathcal{J}^{\bar{v}}
$$

where $C$ is the following tensor

$$
C=\sum_{\alpha} \mathcal{J}^{\alpha} \otimes \mathcal{J}^{\bar{\alpha}}
$$

\section{New linear dilaton backgrounds of Heterotic strings}

These left-coset superconformal field theories can be used to construct various supersymmetric exact string backgrounds. The first class are generalizations of Gepner models [Gep88] and Kazama-Suzuki constructions [KS89b] using the left cosets as building blocks for the internal SCFT. This has already been considered in [BJKZ96] for the $S^{2}$ coset but can be extended using the new theories constructed above. In this case there is no geometric interpretation from the sigma model point of view since these theories have no semi-classical limit. Indeed the levels of the cosets are frozen because their central charge must add up to $c=9$ (in the case of four-dimensional compactification). However we expect that they correspond to special points in the moduli spaces of supersymmetric compactifications, generalizing the Gepner points of the CY manifolds.

Another type of models are the left cosets analogues of the NS5-branes solutions [CHS91, KPR91] and of their extensions to more generic supersymmetric vacua with a dilaton background. It was shown in [GKP99] that a large class of these linear dilaton theories are dual to singular CY manifolds in the decoupling limit. An extensive review of the different possibilities in various dimensions has been given in [ESY03] with all the possible $G / H$ cosets. The left cosets that we constructed allows to extend all these solutions to heterotic strings, with a different geometrical interpretation since our cosets differ from ordinary gauged WZW model. However the superconformal structure of the left sector of our models is exactly the same as for the corresponding gauged WZW - except that the values of the $N=2$ R-charges that appear in the spectrum are constrained - so we can carry over all the known constructions to the case of the geometric cosets.

\footnotetext{
${ }^{18}$ To be precise one could define a $B$ field but this would have to be closed

${ }^{19}$ The dilaton would basically measure the difference between the asymmetric coset volume form and the homogeneous space one as it is shown in [Tse94]
} 
In the generic case these constructions involve non-abelian cosets, and as we showed the asymmetric deformations and gaugings apply only to the abelian components. Thus in general we will get mixed models which are gauged WZW models w.r.t. the non-abelian part of $H$ and geometric cosets w.r.t. the abelian components of $H$. Below we will focus on purely abelian examples, i.e. corresponding to geometric cosets. The dual interpretation of these models, in terms of the decoupling limit of some singular compactification manifolds, is not known. Note however that by construction there are about $\sqrt{k}$ times less massless states in our models than in the standard left-right symmetric solutions. Therefore they may correspond to some compactifications with fluxes, for which the number of moduli is reduced. It would be very interesting to investigate this issue further.

Six-dimensional model. Let us take as a first example the critical superstring background:

$$
\mathbb{R}^{5,1} \times \frac{S L(2, \mathbb{R})_{k+2} \times S O(2)_{1}}{U(1)_{k}} \times\left[U(1)_{k} \backslash S U(2)_{k-2} \times S O(2)_{1}\right]
$$

the first factor is an ordinary gauged model while the second one is a left coset CFT as discussed in this paper. This is the direct analogue of the five-brane solution, or more precisely of the double scaling limit of NS5-branes on a circle [GK99, IKPT04], in the present case with magnetic flux. This theory has $N=2$ charges but, in order to achieve spacetime supersymmetry one must project onto odd-integral $N=2$ charges on the left-moving side, as in the type II construction [IKPT04]. This can be done in the standard way by orbifoldizing the left $N=2$ charges of the two cosets.

Four-dimensional model. A simple variation of the six-dimensional theory is provided by

$$
\begin{aligned}
\mathbb{R}^{3,1} \times \frac{S L(2, \mathbb{R})_{k / 2+2} \times S O(2)_{1}}{U(1)_{2 k}} & \times\left[U(1)_{k} \backslash S U(2)_{k-2} \times S O(2)_{1}\right] \\
& \times\left[U(1)_{k} \backslash S U(2)_{k-2} \times S O(2)_{1}\right]
\end{aligned}
$$

which is the magnetic analogue of the (double scaling limit of) intersecting five-branes solution. Also here an orbifoldization of the left $N=2$ charges is needed to achieve space-time supersymmetry.

Three-dimensional models: the flagbrane ${ }^{\odot}$. We can construct the following background of the $G_{2}$ holonomy type, as in the case of symmetric coset [ES01]:

$$
\mathbb{R}^{2,1} \times \mathbb{R}_{Q} \times\left[U(1)_{k} \times U(1)_{3 k} \backslash S U(3)_{k-3} \times S O(6)_{1}\right]
$$

and the non-trivial part of the metric is

$$
d s^{2}=-\mathrm{d} t^{2}+\mathrm{d} x^{2}+\mathrm{d} y^{2}+\frac{k}{4 r^{2}}\left[\mathrm{~d} r^{2}+4 r^{2} \mathrm{~d} s^{2}\left(S U(3) / U(1)^{2}\right)\right] .
$$


Without the factor of four it would be a direct analogue of the NS5-brane, being conformal to a cone over the flag space.

Another possibility in three dimensions is to lift the $S L(2, \mathbb{R}) / U(1)$ coset to the group manifold $S L(2, \mathbb{R})$. In this case, as for the standard gauged WZW construction [AGS00] we will get the following anti-de Sitter background:

$$
S L(2, \mathbb{R})_{k / 4+2} \times\left[U(1)_{3 k} \backslash S U(3)_{k-3} \times S O(6)_{1}\right]
$$

and the left moving sector of this worldsheet CFT defines an $N=3$ superconformal algebra in spacetime.

Two-dimensional model. In this case we can construct the background:

$$
\mathbb{R}^{1,1} \times \frac{S L(2, \mathbb{R})_{k / 4+2} \times S O(2)_{1}}{U(1)_{4 k}} \times \frac{U(1)_{3 k} S U(3)_{k-3} \times S O(6)_{1}}{U(1)_{k}}
$$

which corresponds in the classification of [ESY03] to a non-compact manifold of $S U(4)$ holonomy once the proper projection is done on the left $N=2$ charges. This solution can be also be thought as conformal to a cone over the Einstein space $S U(3) / U(1)$. Using the same methods as for the NS5branes in [IKPT04], we can show that the full solution corresponding to the model (4.158) can be obtained directly as the null super-coset:

$$
\frac{S L(2, \mathbb{R})_{k / 4} \times U(1) \backslash S U(3)_{k}}{U(1)_{L} \times U(1)_{R}}
$$

where the action is along the elliptic generator in the $S L(2, \mathbb{R})$, with a normalization $\left\langle t^{3}\right\rangle^{2}=-4$, and along the direction $\alpha_{1}+2 \alpha_{2}$ in the coset space $U(1) \backslash S U(3)$, with a canonical normalization. For $r \rightarrow \infty$ the solution asymptotes the cone but when $r \rightarrow 0$ the strong coupling region is smoothly capped by the cigar. 


\section{CHAPTER 5}

\section{Squashed groups in type II}

In this chapter we start deviating from the preceding ones because we will no longer deal with WZW models but with configurations in which the group manifold geometry is sustained by RR fields. In particular, then, we see how the squashed geometries can be obtained in type II theories by T-dualizing black brane configurations.

$\mathrm{T}$

HE MODELS that we have studied so far are intrinsically heterotic. In fact it is the very presence of a heterotic electromagnetic field that allows for the solution of the equations of motion. On the other hand, and this is especially true for purely asymmetric deformations that only have a constant dilaton, we can expect them to be mapped via S-duality to type II solutions. In this chapter we will build such solutions but using a slightly different path: in particular we will see how using a T-duality it is possible to modify a fibration geometry in the same way as we did before by adding a marginal operator, thus recovering the same geometries as above, but this time in presence of Ramond-Ramond fields.

\section{1 $S L(2, \mathbb{R}) \times S U(2)$ as a D-brane solution}

Up to this point we have considered WZW models for the sake of their selfconsistency. In other words we have used group manifolds as part of critical string backgrounds on the ground of the existence of an underlying CFT. On the other hand, at low energies we should obtain a SUGRA description, so it is plausible that a description for the same geometry is available in terms of diverse ten-dimensional sources.

The starting point is a higher-dimensional generalization of the usual fourdimensional charged black hole. The natural, most symmetric, ansatz for the geometry in presence of a $p$-dimensional extended object (a black $D_{p}$-brane) consists in keeping the Lorentz symmetry in $(p+1)$ dimensions and a spherical symmetry in $(9-p)$. In other words breaking the $S O(1,9)$ symmetry to $S O(1, p) \times S O(9-p)$. Moreover we expect a $C_{[(p+1)]}$ form naturally coupled to the $p$-brane. It is possible to show [Ste98] that the solution also contains a dilaton $\Phi(r)$ and is completely determined in terms of a harmonic function 
$H_{p}(r):$

$$
\left\{\begin{array}{l}
\mathrm{d} s^{2}=H_{p}(r)^{-1 / 2}\left(-\mathrm{d} t^{2}+\mathrm{d} x_{1}^{2}+\ldots+\mathrm{d} x_{p}^{2}\right)+H_{p}(r)\left(\mathrm{d} r^{2}+r^{2} \mathrm{~d} \Omega_{8-p}^{2}\right) \\
C_{[(p+1)]}=\left(1-H_{p}(r)^{-1}\right) \mathrm{d} t \wedge \mathrm{d} x^{1} \wedge \ldots \wedge \mathrm{d} x^{p} \\
e^{\Phi}=H_{p}(r)^{(3-p) / 4}
\end{array}\right.
$$

where $H_{P}(r)$ is explicitly given by

$$
H_{p}(r)=1+\frac{Q_{p}}{r^{7-p}}
$$

More complicated solutions with intersecting branes can be studied and in particular the solution for a D1-D5 system reads:

$$
\left\{\begin{array}{c}
\begin{array}{rl}
\mathrm{d} s^{2}=H_{1}(r)^{-1 / 2} H_{5}(r)^{-1 / 2}\left(-\mathrm{d} t^{2}+\mathrm{d} x_{1}^{2}\right)+H_{1}(r)^{1 / 2} H_{5}(r)^{-1 / 2}\left(\mathrm{~d} x_{2}^{2}+\ldots+\mathrm{d} x_{5}^{2}\right)+ \\
\quad+H_{1}(r)^{1 / 2} H_{5}(r)^{1 / 2}\left(\mathrm{~d} r^{2}+\mathrm{d} \Omega_{3}^{2}\right)
\end{array} \\
C_{[2]}=\left(1-H_{1}(r)^{-1}\right) \mathrm{d} t \wedge \mathrm{d} x^{1} \quad \\
C_{[6]}=\left(1-H_{5}(r)^{-1}\right) \mathrm{d} t \wedge \mathrm{d} x^{1} \wedge \ldots \wedge \mathrm{d} x^{5} \\
e^{\Phi}=H_{1}(r)^{1 / 2} H_{5}(r)^{-1 / 2}
\end{array}\right.
$$

where in this case both $H_{1}(r)$ and $H_{5}(r)$ have the same $1 / r^{2}$ behaviour. It is then simple to see that in the near-horizon limit, i.e. for $r \rightarrow 0$, the geometry we find is $\mathrm{AdS}_{3} \times S^{3} \times \mathbb{R}^{4}$, or $S L(2, \mathbb{R}) \times S U(2)$, plus four flat directions.

\subsection{T duality with RR fields}

In the IIB solutions we consider in this section the rôle of sustaining the geometry previously held by the Kalb-Ramond three-form is taken by RR field strengths. This has a number of consequences, first of all the lack of a proper CFT description for such configurations. In particular this means also that the usual Buscher rules [Bus87] prove insufficient and we are forced to follow a slightly more involved path to write T-duals: derive two low-energy effective actions and explicitly write the transformations relating them (in this we will follow the same procedure as in [DLP98, DLP99]).

Type II action in nine dimensions
In ten dimensions, type IIA and IIB are related by a T-duality transformation, stating that the former theory compactified on a circle of radius $R$ is equivalent to the latter on a circle of radius $1 / R$. This means in particular that there is only one possible nine-dimensional $N=2$ SUGRA action. The rules of T-duality are then easily obtained by explicitly writing the two low-energy actions and identifying the corresponding terms.

For sake of clarity let us just consider the bosonic sector of both theories. In [LP96, LPS96] it was found that the IIA action in nine dimensions is given 
by

$$
\begin{aligned}
e^{-1} L_{I I A}=R-\frac{1}{2}(\partial \phi)^{2}- & \frac{1}{2}(\partial \varphi)^{2}-\frac{1}{2}\left(\mathcal{F}_{[1]}^{(12)}\right)^{2} e^{\frac{3}{2} \phi+\frac{\sqrt{7}}{2} \varphi}+ \\
-\frac{1}{48}\left(F_{[4]}\right)^{2} e^{\frac{1}{2} \phi+\frac{3}{2 \sqrt{7}} \varphi}- & \frac{1}{12}\left(F_{[3]}^{(1)}\right)^{2} e^{-\phi+\frac{1}{\sqrt{7}} \varphi}-\frac{1}{12}\left(F_{[3]}^{(2)}\right)^{2} e^{\frac{1}{2} \phi-\frac{5}{2 \sqrt{7}} \varphi}+ \\
-\frac{1}{4}\left(F_{[2]}^{(12)}\right)^{2} e^{-\phi-\frac{3}{\sqrt{7}} \varphi}- & \frac{1}{4}\left(\mathcal{F}_{[2]}^{(1)}\right)^{2} e^{\frac{3}{2} \phi+\frac{1}{2 \sqrt{7}} \varphi}-\frac{1}{4}\left(\mathcal{F}_{[2]}^{(2)}\right)^{2} e^{\frac{4}{\sqrt{7}} \varphi}+ \\
& -\frac{1}{2 e} \tilde{F}_{[4]} \wedge \tilde{F}_{[4]} \wedge A_{[1]}^{(12)}-\frac{1}{e} \tilde{F}_{[3]}^{(1)} \wedge \tilde{F}_{[3]}^{(2)} \wedge A_{[3]},
\end{aligned}
$$

where $\phi$ is the original dilaton, $\varphi$ is a scalar measuring the compact circle, defined by the reduction (in string frame)

$$
\mathrm{d} s^{2}=e^{\phi / 2} \mathrm{~d} s_{10}^{2}=e^{\phi / 2}\left(e^{-\varphi /(2 \sqrt{7})} \mathrm{d} s_{9}^{2}+e^{\sqrt{7} \varphi / 2}\left(\mathrm{~d} z+\mathcal{A}_{[1]}\right)^{2}\right)
$$

and $F_{[n]}$ are $n$-form field strengths defined as

$$
\begin{aligned}
F_{[4]} & =\tilde{F}_{[4]}-\tilde{F}_{[3]}^{(1)} \wedge \mathcal{A}_{[1]}^{(1)}-\tilde{F}_{[3]}^{(2)} \wedge \mathcal{A}_{[1]}^{(2)}-\frac{1}{2} \tilde{F}_{[2]}^{(12)} \wedge \mathcal{A}_{[1]}^{(1)} \wedge \mathcal{A}_{[1]}^{(2)} \\
F_{[3]}^{(1)} & =\tilde{F}_{[3]}^{(1)}-\tilde{F}_{[2]}^{(12)} \wedge \mathcal{A}_{[1]}^{(2)} \\
F_{[3]}^{(2)} & =\tilde{F}_{[3]}^{(2)}+F_{[2]}^{(12)} \wedge \mathcal{A}_{[1]}^{(1)}-\mathcal{A}_{[0]}^{(12)}\left(\tilde{F}_{[3]}^{(1)}-F_{[2]}^{(12)} \wedge \mathcal{A}_{[1]}^{(2)}\right) \\
F_{[2]}^{(12)} & =\tilde{F}_{[2]}^{(12)} \\
\mathcal{F}_{[2]}^{(1)} & =\mathcal{F}_{[2]}^{(1)}+\mathcal{A}_{[0]}^{(12)} \mathcal{F}_{[1]}^{(2)} \\
\mathcal{F}_{[2]}^{(2)} & =\tilde{\mathcal{F}}_{[2]}^{(2)} \\
\mathcal{F}_{[1]}^{(12)} & =\tilde{\mathcal{F}}_{[1]}^{(12)} .
\end{aligned}
$$

In the same way, starting from the IIB action one obtains the following ninedimensional IIB Lagrangian:

$$
\begin{gathered}
e^{-1} L_{I I B}=R-\frac{1}{2}(\partial \phi)^{2}-\frac{1}{2}(\partial \varphi)^{2}-\frac{1}{2} e^{2 \phi}(\partial \chi)^{2}+ \\
-\frac{1}{48} e^{-\frac{2}{\sqrt{7}} \varphi} F_{[4]}^{2}-\frac{1}{12} e^{-\phi+\frac{1}{\sqrt{7}} \varphi}\left(F_{[3]}^{(\mathrm{NS})}\right)^{2}-\frac{1}{2} e^{\phi+\frac{1}{\sqrt{7}} \varphi}\left(F_{[3]}^{(\mathrm{R})}\right)^{2}+ \\
-\frac{1}{4} e^{\frac{4}{\sqrt{7}} \varphi}\left(\mathcal{F}_{[2]}\right)^{2}-\frac{1}{4} e^{\phi-\frac{3}{\sqrt{7}} \varphi}\left(F_{[2]}^{(\mathrm{R})}\right)^{2}-\frac{1}{4} e^{-\phi-\frac{3}{\sqrt{7}} \varphi}\left(F_{[2]}^{(\mathrm{NS})}\right)^{2}+ \\
-\frac{1}{2 e} \tilde{F}_{[4]} \wedge \tilde{F}_{[4]} \wedge \mathcal{A}_{[1]}-\frac{1}{e} \tilde{F}_{3}^{(\mathrm{NS})} \wedge \tilde{F}_{[3]}^{(\mathrm{R})} \wedge A_{[3]} .
\end{gathered}
$$

Knowing that both describe the same theory we easily obtain the conversion table in Tab. 5.1 which acts as a dictionary between IIA and IIB in ten dimensions plus the following relation between the scalar fields

$$
\left(\begin{array}{c}
\phi \\
\varphi
\end{array}\right)_{I I A}=\left(\begin{array}{cc}
3 / 4 & -\sqrt{7} / 4 \\
-\sqrt{7} / 4 & -3 / 4
\end{array}\right)\left(\begin{array}{l}
\phi \\
\varphi
\end{array}\right)_{\text {IIB }}
$$

This completes the T-duality relations generalizing the usual ones [Bus87] valid in the NS-NS sector. 


\begin{tabular}{|c|c|c|c|c|c|}
\hline & \multicolumn{2}{|c|}{ IIA } & & \multicolumn{2}{|c|}{ IIB } \\
\hline & $D=10$ & $D=9$ & T-duality & $D=9$ & $D=10$ \\
\hline \multirow{4}{*}{$\begin{array}{l}\text { R-R } \\
\text { fields }\end{array}$} & \multirow[t]{2}{*}{$\overline{\overline{A_{3}}}$} & $\overline{A_{3}}$ & $\longleftrightarrow$ & $A_{3}$ & $B_{4}$ \\
\hline & & $A_{2}^{(2)}$ & $\longleftrightarrow$ & $A_{2}^{\mathrm{R}}$ & \multirow[t]{2}{*}{$A_{2}^{\mathrm{R}}$} \\
\hline & \multirow[t]{2}{*}{$\mathcal{A}_{1}^{(1)}$} & $\mathcal{A}_{1}^{(1)}$ & $\longleftrightarrow$ & $A_{1}^{\mathrm{R}}$ & \\
\hline & & $\mathcal{A}_{0}^{(12)}$ & $\longleftrightarrow$ & $\chi$ & $x$ \\
\hline \multirow{3}{*}{$\begin{array}{l}\text { NS-NS } \\
\text { fields }\end{array}$} & $G_{\mu \nu}$ & $\mathcal{A}_{1}^{(2)}$ & $\longleftrightarrow$ & 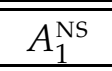 & \multirow[t]{2}{*}{$A_{2}^{\mathrm{NS}}$} \\
\hline & \multirow[t]{2}{*}{$A_{2}^{(1)}$} & $A_{2}^{(1)}$ & $\longleftrightarrow$ & $A_{2}^{\mathrm{NS}}$ & \\
\hline & & $A_{1}^{(12)}$ & $\longleftrightarrow$ & $\mathcal{A}_{1}$ & $G_{\mu v}$ \\
\hline
\end{tabular}

Table 5.1: T-duality dictionary with RR fields

\subsection{The squashed sphere}

Start with a $D_{1}-D_{5}$ system in type IIB described in Sec. 5.1. The near-horizon geometry is

$$
\mathrm{d} s_{10}^{2}=\mathrm{AdS}_{3} \times S^{3} \times \mathbb{R}^{4},
$$

with a three-form flux

$$
F_{3}=\sqrt{2} m\left(\omega_{\mathrm{AdS}}+\omega_{S}\right),
$$

where $\omega$ is the volume form ans the constant $m$ is fixed by demanding:

$$
\begin{aligned}
\left.R i c\right|_{\mathrm{AdS}} & =-\left.m^{2} g\right|_{\mathrm{AdS}} \\
\left.R i c\right|_{S} & =\left.m^{2} g\right|_{S} .
\end{aligned}
$$

Now, introduce the coordinates $(\vartheta, \varphi, \psi, x)$ on $S^{3} \times S^{1}$ and write explicitly:

$$
\begin{aligned}
\mathrm{d} s_{10}^{2} & =\mathrm{AdS}_{3} \times \mathbb{R}^{3}+\frac{1}{2 m^{2}}\left[\mathrm{~d} \vartheta^{2}+\mathrm{d} \varphi^{2}+\mathrm{d} \psi^{2}+2 \cos \vartheta \mathrm{d} \varphi \mathrm{d} \psi\right]+\mathrm{d} x^{2} \\
F_{3} & =m \sqrt{2} \omega_{\text {AdS }}+\frac{\sin \vartheta}{2 m^{2}} \mathrm{~d} \vartheta \wedge \mathrm{d} \varphi \wedge \mathrm{d} \psi,
\end{aligned}
$$

where $x$ is a periodic variable with period

$$
x \sim x+\frac{4 \pi}{\lambda} n .
$$

If we change the variable $\psi$ to $\psi=\alpha+\lambda x$ we still have a $4 \pi$-periodic direction $\alpha$ and can rewrite the metric as:

$$
\begin{aligned}
\mathrm{d} s_{10}^{2} & =\operatorname{AdS}_{3} \times \mathbb{R}^{3}+\frac{1}{2 m^{2}}\left[\mathrm{~d} \vartheta^{2}+\mathrm{d} \varphi^{2}+\mathrm{d} \alpha^{2}+2 \cos \vartheta \mathrm{d} \varphi \mathrm{d} \alpha\right]+\left(1+\frac{\lambda^{2}}{2 m^{2}}\right) \mathrm{d} x^{2}+ \\
& +\frac{\lambda}{m^{2}}(\mathrm{~d} \alpha+\cos \vartheta \mathrm{d} \varphi) \mathrm{d} x \\
F_{3} & =m \sqrt{2} \omega_{\text {AdS }}+\frac{\sin \vartheta}{2 m^{2}} \mathrm{~d} \vartheta \wedge \mathrm{d} \varphi \wedge \mathrm{d} \alpha+\lambda \frac{\sin \vartheta}{2 m^{2}} \mathrm{~d} \vartheta \wedge \mathrm{d} \varphi \wedge \mathrm{d} x
\end{aligned}
$$


Redefining

$$
z=\sqrt{1+\frac{\lambda^{2}}{2 m^{2}}} x \quad h=\frac{\lambda}{2 m} \frac{1}{\sqrt{1+\lambda^{2} / 2 m^{2}}}
$$

the fields read

$$
\begin{aligned}
\mathrm{d} s_{10}^{2} & =\mathrm{AdS}_{3} \times \mathbb{R}^{3}+\frac{1}{2 m^{2}}\left[\mathrm{~d} \vartheta^{2}+\sin ^{2} \vartheta \mathrm{d} \varphi^{2}+\left(1-2 h^{2}\right)(\mathrm{d} \alpha+\cos \vartheta \mathrm{d} \varphi)^{2}\right]+ \\
& +\left[\mathrm{d} z+\frac{h}{m}(\mathrm{~d} \alpha+\cos \vartheta \mathrm{d} \varphi)\right]^{2} \\
F_{3} & =m \sqrt{2} \omega_{\text {AdS }}+\frac{\sin \vartheta}{2 m^{2}} \mathrm{~d} \vartheta \wedge \mathrm{d} \varphi \wedge \mathrm{d} \alpha+\frac{h}{m} \sin \vartheta \mathrm{d} \vartheta \wedge \mathrm{d} \varphi \wedge \mathrm{d} z
\end{aligned}
$$

and we can perform a Kaluza-Klein reduction on $z$ and go to nine dimensions. The metric reads:

$$
\mathrm{d} s_{9}^{2}=\mathrm{AdS}_{3} \times \mathbb{R}^{3}+\frac{1}{2 m^{2}}\left[\mathrm{~d} \vartheta^{2}+\sin ^{2} \vartheta \mathrm{d} \varphi^{2}+\left(1-2 h^{2}\right)(\mathrm{d} \alpha+\cos \vartheta \mathrm{d} \varphi)^{2}\right],
$$

and the gauge fields are obtained from:

$$
F_{3}=F_{3}^{(3)}+F_{2}^{(3)} \wedge(\mathrm{d} z+A),
$$

where $F_{m}^{(n)}$ is the $m$-form obtained from the reduction of a $n$-form and $A$ is the one-form

$$
A=\frac{h}{m}(\mathrm{~d} \alpha+\cos \vartheta \mathrm{d} \varphi) .
$$

Explicitly, adding the extra Kaluza-Klein two-form:

$$
\begin{aligned}
& F_{3}^{(3)}=m \sqrt{2} \omega_{\text {AdS }}+\left(1-2 h^{2}\right) \frac{\sin \vartheta}{2 m^{2}} \mathrm{~d} \vartheta \wedge \mathrm{d} \varphi \wedge \mathrm{d} \alpha \\
& F_{2}^{(3)}=\frac{h}{m} \sin \vartheta \mathrm{d} \vartheta \wedge \mathrm{d} \varphi \\
& F_{2}^{(g)}=\mathrm{d} A=\frac{h}{m} \sin \vartheta \mathrm{d} \vartheta \wedge \mathrm{d} \varphi .
\end{aligned}
$$

For the moment this is just a rewriting. Now, let us perform a T-duality to go to type IIA. The fields keep their expressions but the interpretation changes according to Tab. 5.1: $F_{3}^{(3)}$ now comes from the reduction of a four-form in ten dimensions, $F_{2}^{(2)}$ from a two-form and $F_{2}^{(g)}$ is now obtained as the result of the reduction of the Kalb-Ramond field:

$$
F_{3}^{(4)}=F_{3}^{(3)} \quad F_{2}^{(2)}=F_{2}^{(3)} \quad F_{2}^{(B)}=F_{3}^{(g)} .
$$


We can oxidise back to ten dimensions and get a IIA background:

$$
\begin{aligned}
\mathrm{d} s_{10}^{2} & =\mathrm{AdS}_{3} \times \mathbb{R}^{3}+\frac{1}{2 m^{2}}\left[\mathrm{~d} \vartheta^{2}+\sin ^{2} \vartheta \mathrm{d} \varphi^{2}+\left(1-2 h^{2}\right)(\mathrm{d} \alpha+\cos \vartheta \mathrm{d} \varphi)^{2}\right]+\mathrm{d} \xi^{2} \\
F_{4} & =F_{3}^{(4)} \wedge \mathrm{d} \xi=\left[m \sqrt{2} \omega_{\mathrm{AdS}}+\left(1-2 h^{2}\right) \frac{\sin \vartheta}{2 m^{2}} \mathrm{~d} \vartheta \wedge \mathrm{d} \varphi \wedge \mathrm{d} \alpha\right] \wedge \mathrm{d} \xi \\
F_{2} & =F_{2}^{(2)}=\frac{h}{m} \sin \vartheta \mathrm{d} \vartheta \wedge \mathrm{d} \varphi \\
H_{3} & =F_{2}^{B} \wedge \mathrm{d} \xi=\frac{h}{m} \sin \vartheta \mathrm{d} \vartheta \wedge \mathrm{d} \varphi \wedge \mathrm{d} \xi
\end{aligned}
$$

It is worthwhile to emphasize that by construction $\alpha$ is $4 \pi$-periodic and then the geometry is the one of a respectable squashed three-sphere. A very similar construction was considered in [DLP99]. In that case, though, the authors start with the same $\mathrm{AdS}_{3} \times S^{3}$ geometry with both RR and NS-NS fields and then by reducing on one of the sphere isometries, find the Lens space $S^{3} / \mathbb{Z}_{p}$ or a squashed version, where $p$ and the squashing depend on the values of the charges. This is clearly an orbifold of the solutions above.

In principle these constructions can be extended to other group manifold geometries (e.g. the obvious choice leading to a squashed $\mathrm{AdS}_{3}$ ) but in any case one should start from a configuration with RR fields (typically S-dual to the WZW models we described in great detail previously), since the absence of NS-NS antisymmetric fields is the key ingredient for the trivialization of the fiber bundle. More general geometries can be obtained by starting with a mixed RR-NS-NS configuration. 


\section{CHAPTER 6}

\section{Out of the conformal point: Renormalization Group Flows}

This chapter is devoted to the study of the relaxation of squashed WZW models further deformed by the insertion of non-marginal operators. The calculation is carried from both the target space and world-sheet points of view, once more highlighting the interplay between the two complementary descriptions. In the last part such techniques are used to outline the connection between the time evolution and the RG-flow which is seen as a large friction limit description; we are hence naturally led to a FRW-type cosmological model.

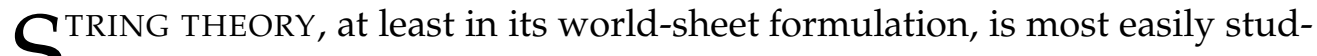
$\mathcal{S}$ ied on-shell. Thanks to the power of conformal field theory, this permits a profound analysis of specific backgrounds. At the same time, though, it makes it difficult to describe more general effects that require a less local knowledge of the theory and its moduli space. In particular it is not obvious how to describe transitions between two different solutions or even the relaxation of an unstable background towards an on-shell solution.

In this chapter we deviate from conformality by adding non-marginal deformations on the top of exact solutions, such as WZW-models or squashedgroup models. The resulting RG-flow then drives those systems back to or away from the conformal point, depending on the character of the deformation. As it is usually the case, these calculations can be faced from two complementary points of view: either in terms of the target space description or in terms of world-sheet two-dimensional theory. We will consider both approaches and show how they do really complete each other, in the sense that they can be considered as two different series expansions of the same quantity. As such, each side contains more information than the other at any given order in perturbation. This will allow us in particular to make a prediction on the outcome of a technically involved one-loop calculation in the WZW and squashed group CFTs on the basis of a two-loop result on target space renormalization.

In the last part of this chapter we use the technology developed so far to show how an RG-flow analysis can allow for further insights on the issue of time-dependent solutions. More precisely we will see how for a given class of 
systems whose geometry is the warped product of a constant curvature space and a time direction the RG-flow equations are a sort of large-friction approximation with the central charge deficit playing the rôle of an effective friction coefficient.

\subsection{The target space point of view}

\section{Renormalization in a dimensional regularization scheme}

Consider the $\sigma$-model with Lagrangian density

$$
\mathcal{L}=\frac{1}{2 \lambda}\left(g_{i j}+B_{i j}\right) \Xi^{i j},
$$

where $g_{i j}$ is a metric, $B_{i j}$ a two-form and $\Xi^{i j}=\partial_{\mu} X^{i} \partial^{\mu} X^{j}+\epsilon_{\mu \nu} \partial^{\mu} X^{i} \partial^{v} X^{j}$. We will say that the model is renormalizable if the corresponding counterterms at any given order in the loop expansion can be reabsorbed into a renormalization of the coupling constant and other parameters that appear in the expressions for $g_{i j}$ and $B_{i j}{ }^{1}$

The standard technique for dealing with this kind of Lagrangian consists in incorporating the Kalb-Ramond field (or, equivalently, the WZ term) into the geometry by reading it as a torsion. This means that instead of the usual Levi-Civita connection one uses the connection $\Gamma^{-}$defined as

$$
\Gamma_{j k}^{-i}=\left\{\begin{array}{c}
i \\
j k
\end{array}\right\}-\frac{1}{2} H_{j k}^{i}
$$

where $\left\{\begin{array}{c}i \\ j k\end{array}\right\}$ is the Christoffel symbol and with respect to this connection one defines the Riemann and Ricci tensors $R^{-}$and $\mathrm{Ric}^{-}$.

Two-loop target space RG-flow

Now, using the background field method in a dimensional regularization scheme (see [Osb90, AGFM81, Fri85, Fri80, BCZ85, HT87] and for various applications [BFHP96, $\left.\left.\mathrm{BFM}^{+} 98, \mathrm{Sfe} 98, \mathrm{Sfe} 99\right]\right)$ we can calculate the one- and two-loop counterterms that turn out to be:

$$
\begin{aligned}
& \mu^{\varepsilon} \mathcal{L}^{(1)}=\frac{1}{\pi \varepsilon} T^{(1)}=\frac{\alpha^{\prime}}{2 \varepsilon \lambda} \operatorname{Ric}^{-}{ }_{i j} \Xi^{i j}, \\
& \mu^{\varepsilon} \mathcal{L}^{(2)}=\frac{\lambda}{8 \pi^{2} \varepsilon} T^{(2)}=\frac{\alpha^{\prime 2}}{16 \varepsilon \lambda} Y^{l m k}{ }_{j} R^{-}{ }_{i k l m} \Xi^{i j},
\end{aligned}
$$

where $Y$ is given by

$$
Y_{i j k l}=-2 R^{-}{ }_{i j k l}+3 R^{-}{ }_{[k i j] l}+\frac{1}{2}\left(H^{2}{ }_{k i} g_{j l}-H^{2}{ }_{k j} g_{i l}\right),
$$

and

$$
H^{2}{ }_{i j}=H_{[3]_{i l m}} H_{[3] j}{ }^{l m} \text {. }
$$

\footnotetext{
${ }^{1}$ When this is not the case the model might nevertheless be renormalizable in a more general sense, in the infinite-dimensional space of metrics and torsions
} 
In general the metric and the Kalb-Ramond field depend on a set of bare parameters $a_{k}^{(0)}$. In this case we can convert the counterterms given above into coupling and parameter renormalizations if we write perturbatively the bare quantities as:

$$
\left\{\begin{array}{l}
\lambda^{(0)}=\mu^{\varepsilon} \lambda\left(1+\frac{J_{1}(a)}{\pi \varepsilon} \lambda+\ldots\right)=\mu^{\varepsilon} \lambda\left(1+\frac{y_{\lambda}}{\epsilon}+\ldots\right), \\
a_{k}^{(0)}=a_{k}+\frac{a_{k}^{(1)}(a)}{\pi \varepsilon}+\ldots=a_{k}\left(1+\frac{y_{a_{k}}}{\epsilon}+\ldots\right), \\
X^{(0) \mu}=X^{\mu}+\frac{X^{(1) \mu}(X, a)}{\pi \varepsilon}+\ldots,
\end{array}\right.
$$

where we allowed for a slight generalization with respect to the definition of renormalization given above in terms of a coordinate reparametrization of the target space ${ }^{2}$. Then the one- and two-loop $\beta$-functions are given by:

$$
\left\{\begin{array}{l}
\beta_{\lambda}=\frac{\mathrm{d} \lambda}{\mathrm{d} t}=\lambda^{2} \frac{\partial y_{\lambda}}{\partial \lambda}=\frac{\lambda^{2}}{\pi}\left(J^{(1)}(a)+\frac{\lambda}{4 \pi} J^{(2)}(a)\right), \\
\beta_{a_{k}}=\frac{\mathrm{d} a_{k}}{\mathrm{~d} t}=\lambda a_{k} \frac{\partial y_{a_{k}}}{\partial \lambda}=\frac{\lambda}{\pi}\left(a_{k}^{(1)}(a)+\frac{\lambda}{4 \pi} a_{k}^{(2)}(a)\right) .
\end{array}\right.
$$

The unknown functions $J^{(i)}, a^{(i)}, X^{(i) \mu}$ are determined by the equation

$$
T^{(i)}=-J^{(i)} \mathcal{L}+\frac{\partial \mathcal{L}}{\partial a_{k}} a_{k}^{(i)}+\frac{\partial \mathcal{L}}{\partial X^{\mu}} X^{(i) \mu} .
$$

This corresponds to demanding the generalized quantum effective action $\Gamma[X]$ to be finite order by order.

\section{Two-loop $\beta$ equations for a WZW model.}

As we have already announced in Ch. 2, the normalization for the WZ term in a WZW action can be fixed by an RG-flow calculation. This is precisely what we will do in the following at two-loop order using the dimensional regularization scheme outlined above. In this way we will find a new apparent non-trivial solution that doesn't show up at first order (and which will prove to be an artifact as we'll see in the following, by using a CFT description in Sec. 6.2). Moreover we will see how the roles of the IR and UV limits are interchanged between the compact and the non-compact case, ie how the same kind of deformation is relevant or irrelevant depending on the compactness of the starting model.

Consider the following action

$$
S_{\lambda, H}=\frac{1}{2 \lambda} \int_{\Sigma} \mathrm{d}^{2} z\left(g_{a b}+\mathrm{H} B_{a b}\right) J_{\mu}^{a} J_{v}^{b} \partial X^{\mu} \bar{\partial} X^{v}
$$

where $J^{a}$ are the Maurer-Cartan one-forms for a group $G$ whose algebra has structure constants $f_{a b c}, g_{a b}=-1 /\left(2 g^{*}\right) f_{a s}^{t} f_{b t}^{s}\left(g^{*}\right.$ is the dual Coxeter number)

\footnotetext{
${ }^{2}$ This redefinition is in general non-linear; in the special case when the $X^{(i)}$ 's depend linearly on $X$ the last equation of the system 6.7 reduces to a multiplicative wavefunction renormalization. The only condition is that $X^{(i)}$ shouldn't depend on the derivatives of $X$. In more geometric terms we are just using the diffeomorphism invariance of the renormalized theory.
} 
and $B_{a b}$ is an antisymmetric matrix satisfying $\mathrm{d}\left(B_{a b} J^{a} \wedge J^{b}\right)=1 / 3 ! f_{a b c} J^{a} \wedge$ $J^{b} \wedge J^{c}$ as in Sec. 2.2. Since the deformation (parameterized by having $\mathrm{H} \neq 1$ ) doesn't affect the geometric part (but for the overall normalization) we can still express the curvature in terms of the Lie algebra structure constants. In particular it is easy to recover that the Riemann tensor is written as:

$$
R_{b c d}^{a}=\frac{1}{4} f_{b e}^{a} f_{c d}^{e}
$$

and the Ricci tensor is obtained by contracting:

$$
R i c_{a b}=\frac{g^{*}}{2} g_{a b}
$$

as in Eqs. (2.65).

In order to write the beta equations as described above we need to incorporate the WZ term (or, more precisely, the Kalb-Ramond field) into the geometry. The most natural approach is to consider $H^{a}{ }_{b c}$ as a torsion and include it in the connection [BCZ85]. We hence define:

$$
\Gamma_{b c}^{-a}=\left\{\begin{array}{c}
a \\
b c
\end{array}\right\}-\frac{1}{2} H_{b c}^{a} .
$$

The covariant derivative of a one-form is then defined as:

$$
\nabla^{-}{ }_{a} V_{b}=\partial_{a} V_{b}-\Gamma^{-c}{ }_{a b} V_{c}=\nabla_{a} V_{b}+\frac{1}{2} H_{a b}^{c} V_{c}
$$

where $\nabla_{a}$ is the covariant derivative with respect to the Levi-Civita connection. Similarly we define the curvature:

$$
\left[\nabla^{-}{ }_{a}, \nabla^{-}{ }_{b}\right] V_{c}=R_{c a b}^{-{ }_{c}^{d}} V_{d}+H_{a b}^{d} \nabla^{-} V_{c}
$$

and it is straightforward to show that:

$$
R^{-}{ }_{a b c d}=R_{a b c d}+\frac{1}{2} \nabla_{c} H_{a b d}-\frac{1}{2} \nabla_{d} H_{a b c}+\frac{1}{4} H_{f a c} H_{d b}^{f}-\frac{1}{4} H_{f a d} H_{c b}^{f} .
$$

Let us now specialize this general relation to our particular deformation. Since $H_{a b c}=\mathrm{H} f_{a b c}$ it is immediate that $\nabla_{a} H_{b c d}=0$ and that the Jacobi identity holds. We then derive:

$$
R^{-}{ }_{a b c d}=\left(1-\mathrm{H}^{2}\right) R_{a b c d},
$$

whence in particular:

$$
\operatorname{Ric}^{-}{ }_{a b}=\left(1-\mathrm{H}^{2}\right) \operatorname{Ric}_{a b}=\left(1-\mathrm{H}^{2}\right) \frac{g^{*}}{2} g_{a b} .
$$

The one-loop counterterm becomes:

$$
T_{a b}^{(1)}=\frac{g^{*}}{4}\left(1-\mathrm{H}^{2}\right) g_{a b} .
$$


The evaluation of the two-loop counterterm is lengthy but straightforward once $R_{a b c d}$ is written in terms of the structure constants. The result is:

$$
T_{a b}^{(2)}=\frac{g^{* 2}}{8}\left(1-\mathrm{H}^{2}\right)\left(1-3 \mathrm{H}^{2}\right) g_{a b} .
$$

Substituting these expressions in Eq. (6.9) (and using the fact that $g_{a b}$ and $\epsilon_{a b}$ are orthogonal) one sees that they become identities for the following choice of parameters:

$$
\left\{\begin{array} { l } 
{ J ^ { ( 1 ) } = - \frac { g ^ { * } } { 4 } ( 1 - \mathrm { H } ^ { 2 } ) } \\
{ a _ { \mathrm { H } } ^ { ( 1 ) } = - \frac { g ^ { * } } { 4 } \mathrm { H } ( 1 - \mathrm { H } ^ { 2 } ) }
\end{array} \quad \left\{\begin{array}{l}
J^{(2)}=-\frac{g^{* 2}}{8}\left(1-\mathrm{H}^{2}\right)\left(1-3 \mathrm{H}^{2}\right) \\
a_{\mathrm{H}}^{(2)}=-\frac{g^{* 2}}{8} \mathrm{H}\left(1-\mathrm{H}^{2}\right)\left(1-3 \mathrm{H}^{2}\right)
\end{array}\right.\right.
$$

corresponding to the following beta equations:

$$
\begin{aligned}
& \beta_{\mathrm{H}}=-\frac{1}{4 \pi} \lambda^{*} \mathrm{H}\left(1-\mathrm{H}^{2}\right)\left(1+\frac{1}{8 \pi} \lambda^{*}\left(1-3 \mathrm{H}^{2}\right)\right) \\
& \beta_{\lambda^{*}}=-\frac{1}{4 \pi} \lambda^{* 2}\left(1-\mathrm{H}^{2}\right)\left(1+\frac{1}{8 \pi} \lambda^{*}\left(1-3 \mathrm{H}^{2}\right)\right)
\end{aligned}
$$

where $\lambda^{*}=g^{*} \lambda$ is the effective coupling constant (this is precisely the fixed parameter in a 't Hooft limit since for a $S U(N)$ group $\left.g^{*}=N\right)$. The difference between a compact and a non-compact group lies in the sign of the dual Coxeter number that is respectively positive/negative. In both cases we remark that $\mathrm{H} / \lambda^{*}$ remains constant, which is a nice check of our construction, since in the notation of Ch. 2 this is just the level of the model that, in the compact case, is quantized and hence is not expected to receive any perturbative correction. On the other hand non perturbative effects do eventually lead to the $k \rightarrow k+g^{*}$ shift which is the reason for the two-loop behaviour of the flow.

Let us analyze the flow in detail:

- The flow diagram for the compact case is drawn in Fig.6.1 where we see the presence of three phases:

- region 1 is the basin of attraction for the WZW model $(z=1)$;

- the points in region 2 describe systems that flow towards asymptotic freedom;

- region 3 seems to be the basin of attraction for a different theory, always with a group manifold geometry but with a differently-normalized WZ term.

only a discrete set of trajectories is allowed and, in particular, region 3 - separated from region 1 by the line $\lambda^{*}=4 \pi \mathrm{H}$ - is only accessible for levels $k<g^{*} / 2$.

- The flow diagram for the non-compact case is drawn in Fig...; again we see three different phases:

- region 1 describe theories flowing to asymptotic freedom; 


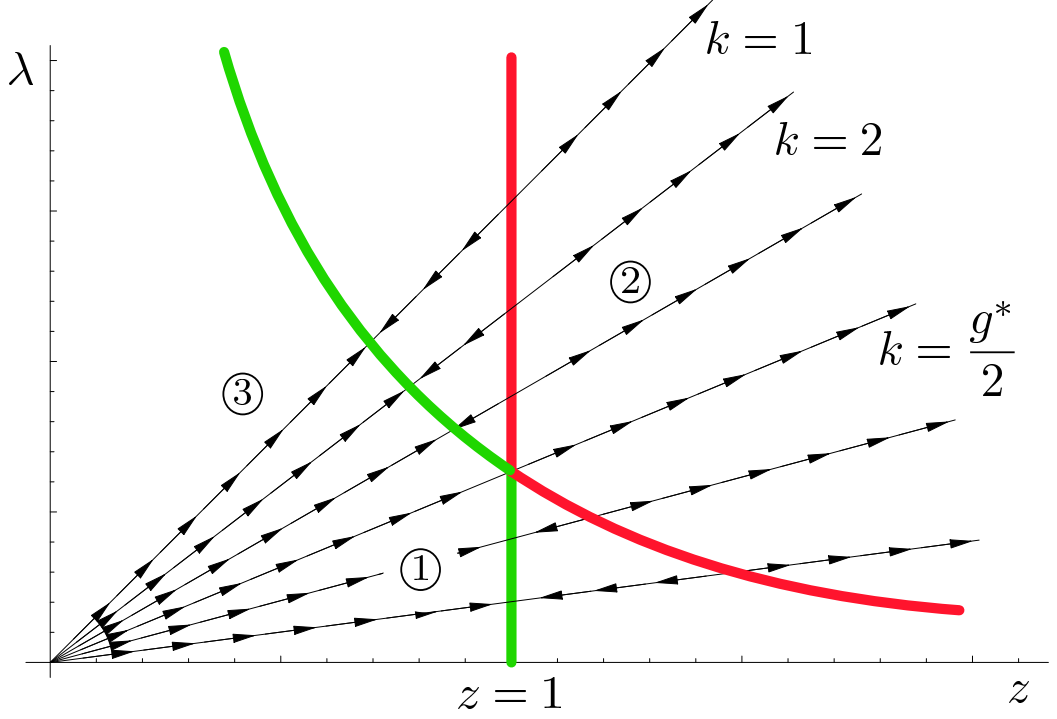

Figure 6.1: Two-loop RG-flow diagram for compact groups.

- region 2 looks like the basin of attraction for the non-trivial solution with the group manifold metric and a new normalization topological term;

- region 3 describe theories flowing to a strong coupling regime.

In particular it is interesting to remark that the roles of the UV and IR are somehow inverted. The WZW model appears as a UV fixed point and thus an unstable solution from the point of view of dynamical systems.

\section{Renormalization group-flow in squashed compact groups}

The models that we have presented in Ch. 3 are conformal; for this reason we expect to find them as fixed points in an RG flow. To verify this claim let us introduce a two-parameter family of $\sigma$ models generalizing the exact backgrounds; a possible choice consists in adding an extra magnetic field on the top of the one responsible for the squashing, but now coming from a higherdimensional right sector. Explicitly

$$
\begin{cases}\mathrm{d} s^{2}=\sum_{\mu \in G / T} J^{\mu} J^{\mu}+\left(1-\mathrm{H}^{2}\right) \sum_{a \in T} J^{a} J^{a}, & \\ H_{[3]}=\frac{\overline{\mathrm{H}}}{2 \mathrm{H}} f_{\mu \nu \rho} J^{\mu} \wedge J^{v} \wedge J^{\rho} & \mu \in G / T, \\ F^{a}=\frac{\mathrm{H}+\mathrm{H}}{2} \sqrt{\frac{k}{k_{g}}} f_{\mu v}^{a} J^{\mu} \wedge J^{v} & \mu \in G / T, a \in T, \\ \bar{F}^{a}=\frac{\mathrm{H}-\overline{\mathrm{H}}}{2} \sqrt{\frac{k}{k_{g}}} f_{\mu v}^{a} J^{\mu} \wedge J^{v} & \mu \in G / T, a \in T\end{cases}
$$


and in particular for $S U(2)$ :

$$
\left\{\begin{array}{l}
\mathrm{d} s^{2}=\mathrm{d} \theta^{2}+\mathrm{d} \psi^{2}+\mathrm{d} \phi^{2}+\cos \theta \mathrm{d} \psi \mathrm{d} \phi-\mathrm{H}^{2}(\mathrm{~d} \psi+\cos \theta \mathrm{d} \phi)^{2} \\
B=\frac{\overline{\mathrm{H}}}{\mathrm{H}} \cos \theta \mathrm{d} \psi \wedge \mathrm{d} \phi \\
A=(\mathrm{H}+\overline{\mathrm{H}})(\mathrm{d} \psi+\cos \theta \mathrm{d} \phi) \\
\bar{A}=(\mathrm{H}-\overline{\mathrm{H}})(\mathrm{d} \psi+\cos \theta \mathrm{d} \phi)
\end{array}\right.
$$

where $\overline{\mathrm{H}}$ is a new parameter, describing the deviation from the conformal point. It is clear that the above background reduces to the one we are used to in the $\overline{\mathrm{H}} \rightarrow \mathrm{H}$ limit. In particular we see that the metric is unchanged, the Kalb-Ramond field has a different normalization and a new field $\bar{A}$ appears. This configuration can be described in a different way: the geometry of a squashed sphere supports two covariantly constant magnetic fields with charge $Q=\mathrm{H}+\overline{\mathrm{H}}$ and $\bar{Q}=\mathrm{H}-\overline{\mathrm{H}}$; the RG flow will describe the evolution of these two charges from a generic $(Q, \bar{Q})$ to $(2 \mathrm{H}, 0)$, while the sum $Q+\bar{Q}=2 \mathrm{H}$ remains constant. In this sense the phenomenon can be interpreted as a charge transmutation of $\bar{Q}$ into $Q$. The conservation of the total charge is in fact a consequence of having chosen a perturbation that keeps the metric and only changes the antisymmetric part of the background.

We can also see the background in Eq.(6.24) from a higher dimensional perspective where only the metric and the Kalb-Ramond field are switched on. Pictorially:

$$
g=\left(\begin{array}{c|c}
g_{\mathrm{WZW}} & \mathrm{H} J_{a} \\
\hline \mathrm{H} J_{a} & 1
\end{array}\right) \quad B=\left(\begin{array}{c|c}
\overline{\overline{\mathrm{H}}} B_{\mathrm{WZW}} & \overline{\mathrm{H}} J_{a} \\
\hline-\overline{\mathrm{H}} J_{a} & 0
\end{array}\right)
$$

where $g_{\mathrm{WZW}}$ and $B_{\mathrm{WZW}}$ are the usual metric and Kalb-Ramond fields for the WZW model on the group G. More explicitly in the SU(2) case:

$$
g=\left(\begin{array}{cccc}
1 & 0 & 0 & 0 \\
0 & 1 & \cos \theta & \mathrm{H} \\
0 & \cos \theta & 1 & \mathrm{H} \cos \theta \\
0 & \mathrm{H} & \mathrm{H} \cos \theta & 1
\end{array}\right) \quad B=\left(\begin{array}{cccc}
0 & 0 & 0 & 0 \\
0 & 0 & \overline{\mathrm{H}} \cos \theta & \overline{\mathrm{H}} \\
0 & -\frac{\overline{\mathrm{H}}}{\mathrm{H}} \cos \theta & 0 & \overline{\mathrm{H}} \cos \theta \\
0 & -\overline{\mathrm{H}} & -\overline{\mathrm{H}} \cos \theta & 0
\end{array}\right)
$$

where the fourth entry represents the bosonized internal current. In particular this clarifies the stated right-sector origin for the new gauge field $\bar{A}$. This higher dimensional formalism is the one we will use in the following RG analysis.

The beta-equations at two-loop order in the expansion in powers of the overall coupling constant $\lambda$ and the field redefinitions for the internal coordi- 
nates $X^{i}$ turn out to be:

$$
\left\{\begin{array}{l}
\beta_{\lambda^{*}}=\frac{\mathrm{d} \lambda^{*}}{\mathrm{~d} t}=-\frac{\lambda^{* 2}}{4 \pi}\left(1-\frac{\overline{\mathrm{H}}^{2}}{\mathrm{H}^{2}}\right)\left(1+\frac{\lambda^{*}}{8 \pi}\left(1-3 \frac{\overline{\mathrm{H}}^{2}}{\mathrm{H}^{2}}\right)\right) \\
\beta_{\mathrm{H}}=\frac{\mathrm{dH}}{\mathrm{d} t}=\frac{\lambda^{*} \mathrm{H}}{8 \pi}\left(1-\mathrm{H}^{2}\right)\left(1-\frac{\overline{\mathrm{H}}^{2}}{\mathrm{H}^{2}}\right)\left(1+\frac{\lambda^{*}}{8 \pi}\left(1-3 \frac{\overline{\mathrm{H}}^{2}}{\mathrm{H}^{2}}\right)\right), \\
\beta_{\overline{\mathrm{H}}}=\frac{\mathrm{d} \overline{\mathrm{H}}}{\mathrm{d} t}=-\frac{\lambda^{*} \overline{\mathrm{H}}}{8 \pi}\left(1+\mathrm{H}^{2}\right)\left(1-\frac{\overline{\mathrm{H}}^{2}}{\mathrm{H}^{2}}\right)\left(1+\frac{\lambda^{*}}{8 \pi}\left(1-3 \frac{\overline{\mathrm{H}}^{2}}{\mathrm{H}^{2}}\right)\right), \\
X^{i}=X^{i}-\frac{\lambda^{*}}{16}\left(1-\mathrm{H}^{2}\right)\left(1-4 \frac{\overline{\mathrm{H}}^{2}}{\mathrm{H}^{2}}+3 \frac{\overline{\mathrm{H}}^{4}}{\mathrm{H}^{4}}\right),
\end{array}\right.
$$

where $\lambda^{*}=\lambda g^{*}, g^{*}$ being the dual Coxeter number, is the effective coupling constant $\left(\lambda^{*}=N \lambda\right.$ for $\left.G=S U(N)\right)$. The contributions at one- and two-loop order are clearly separated. In the following we will concentrate on the oneloop part and we will comment on the two-loop result later. Let us then consider the system:

$$
\left\{\begin{array}{l}
\beta_{\lambda^{*}}=\frac{\mathrm{d} \lambda^{*}}{\mathrm{~d} t}=-\frac{\lambda^{* 2}}{4 \pi}\left(1-\frac{\overline{\mathrm{H}}^{2}}{\mathrm{H}^{2}}\right), \\
\beta_{\mathrm{H}}=\frac{\mathrm{dH}}{\mathrm{d} t}=\frac{\lambda^{*} \mathrm{H}}{8 \pi}\left(1-\mathrm{H}^{2}\right)\left(1-\frac{\overline{\mathrm{H}}^{2}}{\mathrm{H}^{2}}\right), \\
\beta_{\overline{\mathrm{H}}}=\frac{\mathrm{d} \overline{\mathrm{H}}}{\mathrm{d} t}=-\frac{\lambda^{*} \overline{\mathrm{H}}}{8 \pi}\left(1+\mathrm{H}^{2}\right)\left(1-\frac{\overline{\mathrm{H}}^{2}}{\mathrm{H}^{2}}\right) .
\end{array}\right.
$$

This can be integrated by introducing the parameter $z=\overline{\mathrm{H}} / \mathrm{H}$ which makes one of the equations redundant. The other two become:

$$
\left\{\begin{array}{l}
\dot{\lambda}^{*}=-\frac{\lambda^{* 2}}{4 \pi}\left(1-z^{2}\right), \\
\dot{z}=-\frac{\lambda^{*} z}{4 \pi}\left(1-z^{2}\right) .
\end{array}\right.
$$

By inspection one easily sees that $\dot{\lambda} / \lambda=\dot{z} / z$, implying $\lambda(t)=C z(t)$, where $C$ is a constant. This was to be expected since $C$ is proportional to the normalization of the topological WZ term. Since we are dealing with a compact group it turns out that $C$ is, as in [Wit84], quantized with:

$$
C_{k}=\frac{2 \pi}{k}, k \in \mathbb{N}
$$

Now it's immediate to separate the system and find that $z(t)$ is defined as the solution to the implicit equation:

$$
-\frac{t}{2 k}=\frac{1}{z_{0}}-\frac{1}{z(t)}+\log \left[\frac{(z(t)+1)\left(z_{0}-1\right)}{(z(t)-1)\left(z_{0}+1\right)}\right]
$$

with the initial condition $z(0)=z_{0}$. A similar expression was found in [BCZ85, Wit84]. The reason for this is, as pointed out previously [KK95], that the conformal model $(\overline{\mathrm{H}}=\mathrm{H})$ in its higher-dimensional representation (the one in Eq. (6.26)) coincides with a $G \times H$ WZW model after a suitable local field redefinition.

As it is usually the case in the study of non-linear dynamics, a better understanding of the solution is obtained by drawing the RG flow. In a $\left(z, \lambda^{*}\right)$ plane, the trajectories are straight lines through the origin and only a discrete set of them are allowed. Moreover the line $z=1$ is an IR fixed-point locus. This 


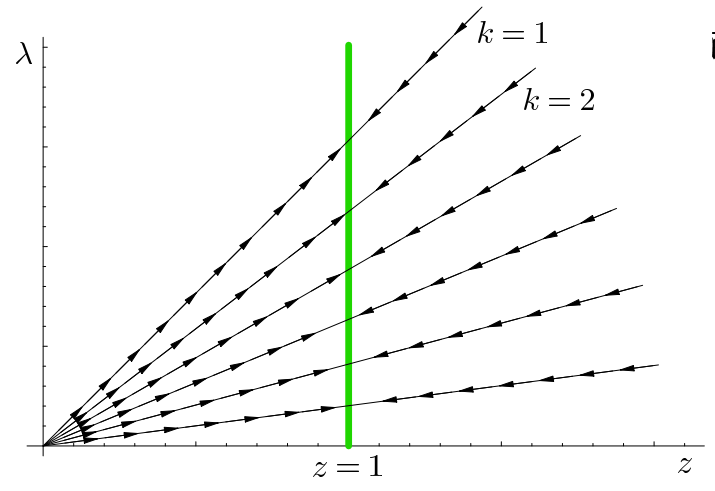

(a) $(z, \lambda)$ plane

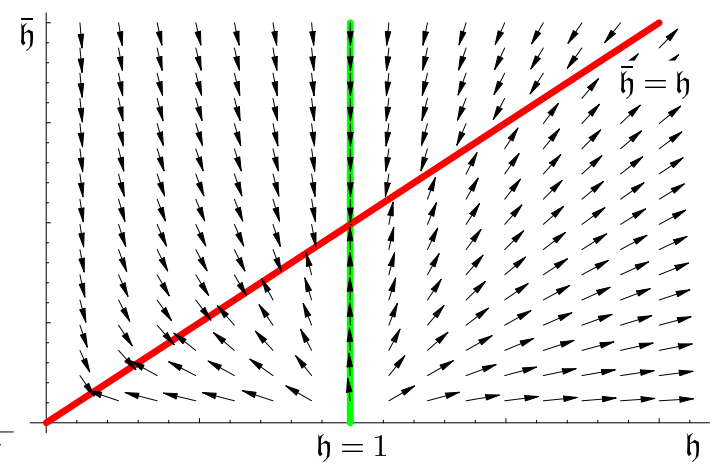

(b) $(\mathrm{H}, \overline{\mathrm{H}})$ plane

Figure 6.2: Flow lines for the deformed (non-conformal) squashed WZW model in $(\mathrm{a})$ the $(z, \lambda)$ and $(\mathrm{b})$ the $(\mathrm{H}, \overline{\mathrm{H}})$ planes. The arrows point in the negative $t$ direction, i.e. towards the infrared; in (a) we see how the squashed WZW model $z=1$ appears as an IR fixed point, in (b) how perturbing the conformal $\overline{\mathrm{H}}=\mathrm{H}$ model by increasing $\overline{\mathrm{H}}$ leads to a a new fixed point corresponding to a value of H closer to 1.

situation is sketched in Fig. 6.2(a). Just as expected the $z=\overline{\mathrm{H}} / \mathrm{H}=1$ point, corresponding to the initial exact model described in Ch. 3 , is an IR fixed point for the RG flow.

Further insights can be gained if we substitute the condition $\lambda^{*}=C_{k} \overline{\mathrm{H}} / \mathrm{H}$ into the system (6.29) thus getting:

$$
\left\{\begin{array}{l}
\frac{\mathrm{dH}}{\mathrm{d} t}=\frac{\overline{\mathrm{H}}}{4 k}\left(1-\mathrm{H}^{2}\right)\left(1-\frac{\overline{\mathrm{H}}^{2}}{\mathrm{H}^{2}}\right), \\
\frac{\mathrm{d} \overline{\mathrm{H}}}{\mathrm{d} t}=-\frac{\overline{\mathrm{H}}^{2}}{4 k \mathrm{H}}\left(1+\mathrm{H}^{2}\right)\left(1-\frac{\overline{\mathrm{H}}^{2}}{\mathrm{H}^{2}}\right) .
\end{array}\right.
$$

The flow diagram for this system in the $(H, \bar{H})$ plane, Fig. 6.2(b), shows how the system relaxes to equilibrium after a perturbation. In particular we can see how increasing $\overline{\mathrm{H}}$ leads to a new fixed point corresponding to a value of $\mathrm{H}$ closer to 1.

We would like to pause for a moment and put the above results in perspective. Consider for simplicity the $S U(2)$ case: the target-space of the sigmamodel under consideration is a squashed three-sphere with two different magnetic fields. Along the flow, a transmutation of the two magnetic charges occurs: the system is driven to a point where one of the magnetic charges vanishes. This fixed point is an ordinary squashed-WZW (of the type studied in Ch. 3), that supports a single magnetic charge.

As we pointed out in Ch. 3, in the squashed-WZW, the magnetic field is bounded by a critical value, $H=1$. As long as $H \leq 1$, the geometry is a genuine squashed three-sphere. For $\mathrm{H}>1$, the signature becomes Lorentzian and the geometry exhibits closed time-like curves. Although of limited physical interest, such a background can be used as a laboratory for investigating the 
fate of chronological pathologies along the lines described above. In the case under consideration and under the perturbation we are considering the model shows a symmetry between the $\mathrm{H}>1$ and $\mathrm{H}<1$ regions. The presence of closed time-like curves doesn't seem to effect the stability (note that regions with different signatures are disconnected, i.e. the signature of the metric is preserved under the RG flow). It is clear however that these results are preliminary. To get a more reliable picture for the fate of closed time-like curves, one should repeat the above analysis in a wider parameter space, where other RG motions might appear and deliver a more refined stability landscape.

A final remark concerns the fact that we find the same RG flow behaviour as for a compact (non-squashed) group. We have already made extensive use of the fact that formally the squashed $S U(2)$ behaves like a $S U(2) \times U(1)$ WZW model, in particular in Sec. 3.4 where this was at the root of the norenormalization theorem. In some sense, then, the present calculation is just a perturbative confirmation of that statement.

\section{Renormalization group-flow in squashed anti de Sitter}

As we've already discussed in Sec. 4.2, sigma models based on non-compact group offer richer (i.e. more complex) phase diagrams than the compact ones. In our particular models this is because the possible choices for a Cartan torus are not pairwise conjugated by inner automorphisms and this is why different choices correspond to inequivalent backgrounds, exhibiting different physical properties. If we concentrate our attention on the $S L(2, \mathbb{R})$ WZW model (that is the only non-compact case with just one time direction), we see that the three possible choices for the Cartan generator (elliptic, parabolic, hyperbolic) respectively lead to the exact backgrounds we introduced in Sec. 4.2 and we report here for convenience:

$$
\begin{aligned}
& \left\{\begin{array}{l}
\mathrm{d} s^{2}=\mathrm{d} \rho^{2}-\mathrm{d} t^{2}+\mathrm{d} \phi^{2}-2 \sinh \rho \mathrm{d} t \mathrm{~d} \phi-\mathrm{H}^{2}(\mathrm{~d} t+\sinh \rho \mathrm{d} \phi)^{2}, \\
B=\sinh \rho \mathrm{d} t \wedge \mathrm{d} \phi, \\
A=2 \mathrm{H}(\mathrm{d} t+\sinh \rho \mathrm{d} \phi) .
\end{array}\right. \\
& \left\{\begin{array}{l}
\mathrm{d} s^{2}=\frac{\mathrm{d} u^{2}}{u^{2}}+\frac{\mathrm{d} x^{+} \mathrm{d} x^{-}}{u^{2}}-\mathrm{H}^{2} \frac{\mathrm{d} x^{+} \mathrm{d} x^{+}}{u^{4}}, \\
B=\frac{\mathrm{d} x^{+} \wedge \mathrm{d} x^{-}}{u^{2}}, \\
A=2 \mathrm{H} \frac{\mathrm{d} x^{+}}{u^{2}} .
\end{array}\right. \\
& \left\{\begin{array}{l}
\mathrm{d} s^{2}=\mathrm{d} r^{2}+\mathrm{d} x^{2}-\mathrm{d} \tau^{2}+2 \sinh r \mathrm{~d} x \mathrm{~d} \tau-\mathrm{H}^{2}(\mathrm{~d} x+\sinh r \mathrm{~d} \tau)^{2} \\
B=\sinh r \mathrm{~d} x \wedge \mathrm{d} \tau, \\
A=2 \mathrm{H}(\mathrm{d} x+\sinh r \mathrm{~d} \tau) .
\end{array}\right.
\end{aligned}
$$

Since these solutions are exact CFT backgrounds, we expect them to appear as fixed points for an RG flow, like the compact configuration described in the previous section. As we will see in the following this is actually the case, but with a difference regarding the role of the UV and IR which is proper to non-compact groups (as explained in Sec. 6.1). Using the same technique as 
above, the first step consists in generalizing the three backgrounds by introducing the following three families of low energy configurations:

$$
\begin{aligned}
& \left\{\begin{array}{l}
\mathrm{d} s^{2}=\mathrm{d} \rho^{2}-\mathrm{d} t^{2}+\mathrm{d} \phi^{2}-2 \sinh \rho \mathrm{d} t \mathrm{~d} \phi-\mathrm{H}^{2}(\mathrm{~d} t+\sinh \rho \mathrm{d} \phi)^{2} \\
B=\frac{\overline{\mathrm{H}}}{\mathrm{H}} \sinh \rho \mathrm{d} t \wedge \mathrm{d} \phi \\
A=(\mathrm{H}+\overline{\mathrm{H}})(\mathrm{d} t+\sinh \rho \mathrm{d} \phi) \\
\bar{A}=(\mathrm{H}-\overline{\mathrm{H}})(\mathrm{d} t+\sinh \rho \mathrm{d} \phi)
\end{array}\right. \\
& \left\{\begin{array}{l}
\mathrm{d} s^{2}=\frac{\mathrm{d} u^{2}}{u^{2}}+\frac{\mathrm{d} x^{+} \mathrm{d} x^{-}}{u^{2}}-\mathrm{H}^{2} \frac{\mathrm{d} x^{+} \mathrm{d} x^{+}}{u^{4}} \\
B=\frac{\overline{\mathrm{H}}}{\mathrm{H} x^{+} \wedge \mathrm{d} x^{-}} \\
A=(\mathrm{H}+\overline{\mathrm{H}}) \frac{\mathrm{d} x^{+}}{u^{2}} \\
\bar{A}=(\mathrm{H}-\overline{\mathrm{H}}) \frac{\mathrm{d} x^{+}}{u^{2}}
\end{array}\right. \\
& \left\{\begin{array}{l}
\mathrm{d} s^{2}=\mathrm{d} r^{2}+\mathrm{d} x^{2}-\mathrm{d} \tau^{2}+2 \sinh r \mathrm{~d} x \mathrm{~d} \tau-\mathrm{H}^{2}(\mathrm{~d} x+\sinh r \mathrm{~d} \tau)^{2} \\
B=\frac{\overline{\mathrm{H}}}{\mathrm{H}} \sinh r \mathrm{~d} x \wedge \mathrm{d} \tau \\
A=(\mathrm{H}+\overline{\mathrm{H}})(\mathrm{d} x+\sinh r \mathrm{~d} \tau) \\
\bar{A}=(\mathrm{H}-\overline{\mathrm{H}})(\mathrm{d} x+\sinh r \mathrm{~d} \tau)
\end{array}\right.
\end{aligned}
$$

The guiding principle remains the same, i.e. keep the same geometry, rescale the KR field and introduce a new electromagnetic field, coming (in a fourdimensional perspective) from the right-moving sector. Again we will observe the same charge-transmutation effect as before, this time in terms of charge density (or charge at infinity).

The backgrounds above can be equivalently described in four dimensions by a metric and a KR field as follows:

$g=\left(\begin{array}{cccc}1 & 0 & 0 & 0 \\ 0 & -1 & -\sinh \rho & \mathrm{H} \\ 0 & -\sinh \rho & 1 & \mathrm{H} \sinh \rho \\ 0 & \mathrm{H} & \mathrm{H} \sinh \rho & 1\end{array}\right) \quad B=\left(\begin{array}{cccc}0 & 0 & 0 & 0 \\ 0 & 0 & \overline{\mathrm{H}} \sinh \rho & \overline{\mathrm{H}} \\ 0 & -\frac{\overline{\mathrm{H}}}{\mathrm{H}} \sinh \rho & 0 & \overline{\mathrm{H}} \sinh \rho \\ 0 & -\overline{\mathrm{H}} & -\overline{\mathrm{H}} \sinh \rho & 0\end{array}\right)$

$g=\left(\begin{array}{cccc}\frac{1}{u^{2}} & 0 & 0 & 0 \\ 0 & 0 & \frac{1}{2 u^{2}} & \frac{\mathrm{H}}{u^{2}} \\ 0 & -\frac{1}{2 u^{2}} & 0 & 0 \\ 0 & \frac{\mathrm{H}^{2}}{u^{2}} & 0 & 1\end{array}\right) \quad B=\left(\begin{array}{cccc}0 & 0 & 0 & 0 \\ 0 & 0 & \overline{\mathrm{H}} \frac{1}{2 u^{2}} & \frac{\overline{\mathrm{H}}}{u^{2}} \\ 0 & \frac{\overline{\mathrm{H}}}{\mathrm{H}} \frac{1}{2 u^{2}} & 0 & 0 \\ 0 & -\frac{\mathrm{H}}{u^{2}} & 0 & 0\end{array}\right)$

$g=\left(\begin{array}{cccc}1 & 0 & 0 & 0 \\ 0 & 1 & \sinh r & \mathrm{H} \\ 0 & \sinh r & -1 & \mathrm{H} \sinh r \\ 0 & \mathrm{H} & \mathrm{H} \sinh r & 1\end{array}\right) \quad B=\left(\begin{array}{cccc}0 & 0 & 0 & 0 \\ 0 & 0 & \overline{\mathrm{H}} \sinh r & \overline{\mathrm{H}} \\ 0 & -\frac{\overline{\mathrm{H}}}{\mathrm{H}} \sinh r & 0 & \overline{\mathrm{H}} \sinh r \\ 0 & -\overline{\mathrm{H}} & -\overline{\mathrm{H}} \sinh r & 0\end{array}\right)$

We must now evaluate the $R^{-}$tensor (i.e. the Ricci tensor with respect to the connection $\left.\Gamma^{-}=\Gamma+1 / 2 H\right)$ and read the counterterms in a dimensional 
regularization scheme as described in Eq. (6.7):

$$
\left\{\begin{array} { l } 
{ J ^ { ( 1 ) } = \frac { 1 } { 4 } ( 1 - \frac { \overline { \mathrm { H } } ^ { 2 } } { \mathrm { H } ^ { 2 } } , , } \\
{ a _ { \mathrm { H } } ^ { ( 1 ) } = - \frac { 1 + \mathrm { H } ^ { 2 } } { 8 } \mathrm { H } ( 1 - \frac { \overline { \mathrm { H } } ^ { 2 } } { \mathrm { H } ^ { 2 } } ) , } \\
{ a _ { \overline { \mathrm { H } } } ^ { ( 1 ) } = \frac { 1 - \mathrm { H } ^ { 2 } } { 8 } \overline { \mathrm { H } } ( 1 - \frac { \overline { \mathrm { H } } ^ { 2 } } { \mathrm { H } ^ { 2 } } ) , } \\
{ X _ { X } ^ { ( 1 ) } = \frac { 1 + \mathrm { H } ^ { 2 } } { 8 } ( 1 - \frac { \overline { \mathrm { H } } ^ { 2 } } { \mathrm { H } ^ { 2 } } ) X . }
\end{array} \quad \left\{\begin{array} { l } 
{ J ^ { ( 1 ) } = \frac { 1 } { 4 } ( 1 - \frac { \overline { \mathrm { H } } ^ { 2 } } { \mathrm { H } ^ { 2 } } ) , } \\
{ a _ { \mathrm { H } } ^ { ( 1 ) } = - \frac { 1 } { 8 } \mathrm { H } ( 1 - \frac { \overline { \mathrm { H } } ^ { 2 } } { \mathrm { H } ^ { 2 } } ) , } \\
{ a _ { \overline { \mathrm { H } } } ^ { ( 1 ) } = \frac { 1 } { 8 } \overline { \mathrm { H } } ( 1 - \frac { \overline { \mathrm { H } } ^ { 2 } } { \mathrm { H } ^ { 2 } } ) , } \\
{ X _ { X } ^ { ( 1 ) } = \frac { 1 } { 8 } ( 1 - \frac { \overline { \mathrm { H } } ^ { 2 } } { \mathrm { H } ^ { 2 } } ) X . }
\end{array} \quad \left\{\begin{array}{l}
J^{(1)}=\frac{1}{4}\left(1-\frac{\overline{\mathrm{H}}^{2}}{\mathrm{H}^{2}}\right), \\
a_{\mathrm{H}}^{(1)}=-\frac{1-\mathrm{H}^{2}}{8} \mathrm{H}\left(1-\frac{\overline{\mathrm{H}}^{2}}{\mathrm{H}^{2}}\right), \\
a_{\overline{\mathrm{H}}}^{(1)}=\frac{1+\mathrm{H}^{2}}{8} \overline{\mathrm{H}}\left(1-\frac{\overline{\mathrm{H}}^{2}}{\mathrm{H}^{2}}\right), \\
X_{X}^{(1)}=\frac{1-\mathrm{H}^{2}}{8}\left(1-\frac{\overline{\mathrm{H}}^{2}}{\mathrm{H}^{2}}\right) X .
\end{array}\right.\right.\right.
$$

The analogies among the three configurations are clear, but become striking when we introduce the parameter $z=\overline{\mathrm{H}} / \mathrm{H}$ and all three $\beta$-functions systems all reduce to the following:

$$
\left\{\begin{array}{l}
\dot{\lambda}=\frac{\lambda^{2}}{4 \pi}\left(1-z^{2}\right), \\
\dot{z}=\frac{\lambda z}{4 \pi}\left(1-z^{2}\right) .
\end{array}\right.
$$

This is (up to a sign) the same system we found in the compact case and it is hence immediate to write the solution

$$
\begin{aligned}
& \lambda(t)=C z(t) \\
& \frac{C t}{4 \pi}=\frac{1}{z_{0}}-\frac{1}{z(t)}+\log \left[\frac{(z(t)+1)\left(z_{0}-1\right)}{(z(t)-1)\left(z_{0}+1\right)}\right] .
\end{aligned}
$$

Although, as expected, $z=1$ is a fixed point (corresponding to the conformal points) some differences are important. First of all the background is non-compact, so $C$ is not quantized and, although the flow trajectories are still straight lines through the origin, the angular parameter is now arbitrary. The other difference is that $z=1$ is a fixed point, but it doesn't correspond to a IR stable configuration but to a UV stable one. This is precisely the same behaviour that one encounters for non-compact WZW models when varying the normalization of the WZ term (as in Sec. 6.1). Again the flow diagram is the same as for the original $S L(2, \mathbb{R})$ group and is summarized in Fig. 6.3.

\subsection{The CFT approach}

In order to make contact with genuine CFT techniques, we must identify the relevant operators which are responsible for the $(\mathrm{H}, \overline{\mathrm{H}})$ deformation of the $G \times$ $H$ original WZW model $\left(H=U(1)^{\operatorname{rank} G}\right)$. At lowest approximation, all we need is their conformal dimensions in the unperturbed theory.

Following [Zam86], let $S_{0}$ be the unperturbed (conformal) action and $\mathcal{O}_{i}$ the relevant operators of conformal dimension $\Delta_{i}=1-\epsilon_{i}$. Consider the perturbed model,

$$
S=S_{0}+g^{i} \mathcal{O}_{i} .
$$

The tree-level beta-functions read:

$$
\beta^{i}(g)=-\epsilon_{i} g^{i},
$$




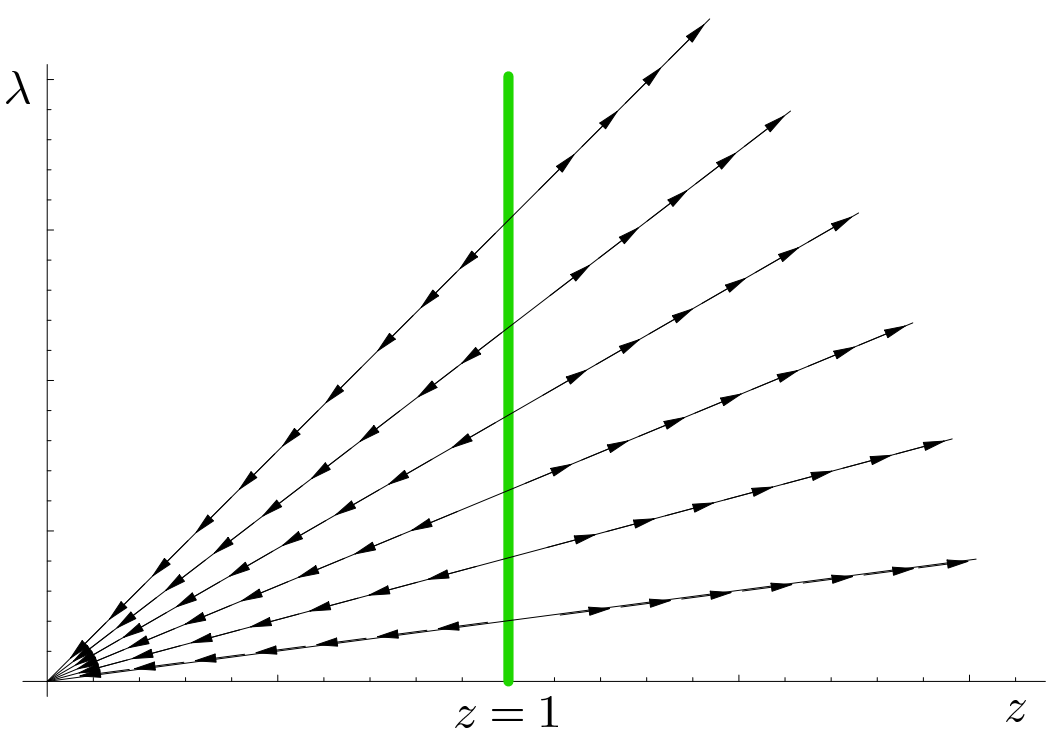

Figure 6.3: Flow diagram for the system in Eq. (6.44). The arrows point from the UV to the IR and $z=1$ appears as a UV stable solution locus.

where $g^{i}$ is supposed to be small, for the perturbative expansion of $\beta^{i}$ to hold ${ }^{3}$.

The $G \times H$ primary operator we need can be written as follows:

$$
\mathcal{O}=\sum_{\mathrm{A}, \mathrm{B}}\left\langle t^{\mathrm{A}} g t^{\mathrm{B}} g^{-1}\right\rangle\left\langle t^{\mathrm{A}} \partial g g^{-1}\right\rangle\left\langle t^{\mathrm{B}} g^{-1} \bar{\partial} g\right\rangle=\sum_{\mathrm{A}, \mathrm{B}} \Phi^{\mathrm{AB}} J^{\mathrm{A}} \bar{J}^{\mathrm{B}},
$$

where $\Phi^{\mathrm{AB}}$ is a primary field transforming in the adjoint representation of the left and right groups $G$. As such, the total conformal dimensions (as we've seen in Sec. 2.1) are

$$
\Delta=\bar{\Delta}=1+\frac{g^{*}}{g^{*}+k}
$$

where $g^{*}$ is the dual Coxeter number and as such the operator is irrelevant (in the infrared).

Specializing this general construction to our case we find that the action for the fields in Eq. (3.17) is:

$S=\frac{k}{4 \pi}\left\{S_{0}+\left(\frac{\mathrm{H}}{\overline{\mathrm{H}}}-1\right) \sum_{\mathrm{A}, \mathrm{B}} \Phi^{\mathrm{AB}} J^{\mathrm{A}} \bar{J}^{\mathrm{B}}+\frac{\mathrm{H}}{\overline{\mathrm{H}}}(\mathrm{H}+\overline{\mathrm{H}}) \sum_{i} J^{a_{i}} \bar{J}^{i}+\frac{\mathrm{H}}{\overline{\mathrm{H}}}(\mathrm{H}-\overline{\mathrm{H}}) \sum_{i, \mathrm{~A}} J^{i} \Phi^{a_{i} \mathrm{~A}} \bar{J}^{\mathrm{A}}\right\}$.

where A runs over all currents, $i$ over the internal currents (in $H$ ) and $J^{a_{i}}$ is the WZW current of the Cartan subalgebra of $G$ coupled to the internal $\bar{J}^{i}$. The

${ }^{3}$ One should be very careful in the choice of signs in these formulae. In [Zam86] the time variable, in fact, describes the evolution of the system towards the infrared and as such it is opposite with respect to the $t=\log \mu$ convention that we used in the previous section (as in [Wit84]). 
extra terms can be interpreted as relevant combinations of operators in the $G \times H$ model. The beta-functions are thus computed following Eq. (6.48):

$$
\left\{\begin{array}{l}
\left.\frac{\mathrm{d}}{\mathrm{d} t} \log \left[\left(\frac{\mathrm{H}}{\overline{\mathrm{H}}}-1\right)\right]\right|_{\overline{\mathrm{H}}=\mathrm{H}}=\frac{g^{*}}{g^{*}+k}=\frac{g^{*}}{k}-\frac{g^{* 2}}{k^{2}}+\mathcal{O}\left(\frac{1}{k^{3}}\right), \\
\left.\frac{\mathrm{d}}{\mathrm{d} t} \log [\overline{\mathrm{H}}(\mathrm{H}+\overline{\mathrm{H}})]\right|_{\overline{\mathrm{H}}=\mathrm{H}}=\mathcal{O}\left(\frac{1}{k^{3}}\right) \\
\left.\frac{\mathrm{d}}{\mathrm{d} t} \log [\overline{\mathrm{H}}(\mathrm{H}-\overline{\mathrm{H}})]\right|_{\overline{\mathrm{H}}=\mathrm{H}}=\frac{g^{*}}{g^{*}+k}=\frac{g^{*}}{k}-\frac{g^{* 2}}{k^{2}}+\mathcal{O}\left(\frac{1}{k^{3}}\right) .
\end{array}\right.
$$

Equations (6.52) agree with the results of the field-theoretical approach (up to the overall normalization), at least in the regime where (6.52) are valid, namely for small $\mathrm{H}$ and $\overline{\mathrm{H}}$ perturbations. But there's more: as pointed out before the conformal model $(\overline{\mathrm{H}}=\mathrm{H})$ is exact because it coincides with a $G \times H$ WZW model after a suitable field redefinition for any value of $\mathrm{H}$. As a consequence the equations remain valid for any finite $\mathrm{H}$. This is reassuring both for the validity of the geometrical approach ${ }^{4}$ and for the conclusions on the stability picture of the models under consideration.

The extra information that we obtain from this calculation is about the interpretation for the two-loop $\beta$-function we described in the previous section. In fact it is now clear that with the target-space approach we just describe the Taylor expansion of the tree-level CFT result:

$$
\frac{g^{*}}{g^{*}+k}=\frac{g^{*}}{k}-\frac{g^{* 2}}{k^{2}}+\mathcal{O}\left(\frac{1}{k^{3}}\right)
$$

This is not surprising since the would-be non-trivial fixed point of the twoloop expansion lay out of the validity range for our approximation. If we really want to go beyond the large $k$ limit, we need to push the analysis from this, CFT, side.

Target space $v s$

CFT renormalization
From the target space view point, the renormalization approach remains valid in the large $k$ limit for any value of $\mathrm{H} / \overline{\mathrm{H}}$. This enables us to use Eq. (6.28) and push (for $k \rightarrow \infty)$ Eq. (6.52) at least to the next leading order in $(1-\mathrm{H} / \overline{\mathrm{H}})$ so to get

$$
\left.\frac{\mathrm{d}}{\mathrm{d} t}\left(\frac{\mathrm{H}}{\overline{\mathrm{H}}}-1\right)\right|_{\overline{\mathrm{H}}=\mathrm{H}}=\left(\frac{g^{*}}{k}-\frac{g^{* 2}}{k^{2}}\right)\left(\frac{\mathrm{H}}{\overline{\mathrm{H}}}-1\right)+\frac{1}{2}\left(-\frac{g^{*}}{k}+7 \frac{g^{* 2}}{k^{2}}\right)\left(\frac{\mathrm{H}}{\overline{\mathrm{H}}}-1\right)^{2}+\ldots
$$

that obviously agrees to first order in the coupling $(\mathrm{H} / \overline{\mathrm{H}}-1)$ with the expression above.

The extra information that we obtain from this calculation is about the interpretation for the two-loop beta-function we described in the previous section. The one-loop corrections to (6.48) are of the form $C_{i j k} g^{i} g^{j}$, where $C_{i j k}$ are related to the three-point function of the unperturbed theory [Zam86]. This coefficient is a measure of the dimension of the operator $\mathcal{O}_{i}$ in the theory perturbed by the set of all operators. Eq.(6.54), based on the target-space

\footnotetext{
${ }^{4}$ There is no doubt on the method itself. It could simply fail to describe the desired phenomenon due to an inappropriate ansatz for the off-criticality excursion in parameter space.
} 
approach, precisely predicts the coefficient of the term $(H / \bar{H}-1)^{2}$ to second order in the $1 / k$-expansion. It seems that such a computation is feasible from the CFT viewpoint at least as a series expansion for large $k$. This would allow for a genuine two-loop comparison of the two methods, and is left for future investigation.

\subsection{RG flow and friction}

It has already been noted in literature [GHMS03] that a deep link exists between the equations of motion and the RG-flow. In an oversimplified toy model one can consider the equations of motion for a system with friction:

$$
\frac{\mathrm{d}^{2} r}{\mathrm{~d} t^{2}}=-V^{\prime}(r)-k \frac{\mathrm{d} r}{\mathrm{~d} t}
$$

Large friction corresponds to the $k \rightarrow \infty$ limit where the dynamics described by this second order equation is well approximated by a first order one:

$$
\frac{\mathrm{d} r}{\mathrm{~d} t^{\prime}}=-V^{\prime}(r), \quad t^{\prime}=\frac{t}{k}
$$

At least in some cases the same link exists between the second order equations of motion and the first order RG flow equation: the latter provide a good approximation for the dynamics of the system in some region of the moduli space. In this section we will provide a class of systems (with constantcurvature metrics and no dilaton) where this can be explicitly verified and the "friction" identified with the expectation value for the dilaton which appears out of equilibrium. More precisely we will consider the RG flow for the coupling constant of the metric with respect to the Kalb-Ramond field and then show that the equations that one obtains in this way are an approximation of those for a system in which the constant is a field depending on an extra time direction.

\section{The RG-flow approach}

As announced above we would like to study the RG-flow for the coupling of the metric in a system without dilaton, that is for the sigma model

$$
S=\frac{1}{2 \lambda} \int \mathrm{d}^{2} z\left(c g_{\mu \nu}+B_{\mu \nu}\right) \partial X^{\mu} \bar{\partial} X^{v}
$$

knowing that for $c=1$ the model is conformal. Using the geometric RG-flow approach developed in Sec. 6.1 we find that Riemann tensor with respect to the connection of Eq. (6.13):

$$
R_{\nu \rho \sigma}^{-\mu}=\left(1-\frac{1}{c^{2}}\right) R_{\nu \rho \sigma}^{\mu}
$$

It follows that the one-loop counterterm is given by

$$
T_{\mu v}=\frac{R}{d}\left(1-\frac{1}{c^{2}}\right) g_{\mu \nu}
$$


where for simplicity we supposed the manifold to be Einstein, which is consistent with the fact that the conformal model with fields $g$ and $B$ doesn't include a dilaton. Hence we immediately find the parameters

$$
J(c)=0 \quad a(c)=\frac{R}{d}\left(1-\frac{1}{c^{2}}\right)
$$

and the corresponding beta equations

$$
\left\{\begin{array}{l}
\beta_{\lambda}=0, \\
\beta_{c}=\frac{\lambda}{\pi} a_{c}=\frac{\lambda R}{d \pi}\left(1-\frac{1}{c^{2}}\right) .
\end{array}\right.
$$

In order to compare this result with what we will find in the following we can write

$$
c(\mu)=e^{2 \sigma(\mu)}
$$

where $\mu$ is the energy scale. Then the energy evolution of $\sigma(\mu)$ (going towards the infrared) gives:

$$
\frac{\mathrm{d} \sigma}{\mathrm{d} \mu}=-\frac{\lambda R}{2 d \pi} e^{-2 \sigma(\mu)}\left(1-e^{-4 \sigma(\mu)}\right)=-V^{\prime}(\sigma(\mu))
$$

which admits the implicit solution

$$
\log \mu=-\frac{1}{4}\left(2 e^{2 \sigma(\mu)}+\log (\tanh \sigma(\mu))\right) .
$$

This is for us the equivalent of Eq. (6.56). Now we move to the $(d+1)$ dimensional spacetime to find the corresponding Eq. (6.55).

\section{Spacetime interpretation}

Equations of motion. As we said above we want to describe the same system by introducing an extra time dimension and reading the coupling as a time-dependent field. In other words we would like to write the equations of motion for the following sigma model:

$$
S=\int \mathrm{d}^{2} z\left[-\partial t \bar{\partial} t+\left(c(t) g_{\mu \nu}+B_{\mu v}\right) \partial X^{\mu} \bar{\partial} X^{\nu}\right]
$$

where, $g$ and $B$ are background fields solving the low-energy string equations of motion. In order to write the equations of motion let us rewrite the $d+1$ dimensional metric in terms of a Weyl rescaling as:

$$
\bar{g}_{\mathrm{MN}}=e^{2 \sigma(t)}\left(\begin{array}{cc}
-e^{-2 \sigma(t)} & 0 \\
0 & g_{\mu \nu}
\end{array}\right)=e^{2 \sigma(t)} g_{\mathrm{MN}}
$$

where $c(t)=e^{2 \sigma(t)}$. This means in particular that the Ricci tensor (this time with respect to the standard Levi-Civita connection) can be written as

$$
\overline{\operatorname{Ric}}_{\mathrm{MN}}=R i C_{\mathrm{MN}}-g_{\mathrm{MN}} K_{\mathrm{L}}^{\mathrm{L}}-(d-1) K_{\mathrm{MN}}
$$


where $K_{\mathrm{MN}}$ is defined as

$$
\begin{aligned}
& K_{\mathrm{M}}{ }^{\mathrm{N}}=-\partial_{\mathrm{M}} \sigma g^{\mathrm{NL}} \partial_{\mathrm{L}} \sigma+g^{\mathrm{NL}}\left(\partial_{\mathrm{M}} \partial_{\mathrm{L}} \sigma-\Gamma^{\mathrm{P}}{ }_{\mathrm{ML}} \partial_{\mathrm{P}} \sigma\right)+\frac{1}{2} g^{\mathrm{LP}} \partial_{\mathrm{L}} \sigma \partial_{\mathrm{P}} \sigma \delta_{\mathrm{M}}{ }^{\mathrm{N}} \\
& K_{\mathrm{MN}}=g_{\mathrm{NL}} B_{\mathrm{M}}{ }^{\mathrm{L}}
\end{aligned}
$$

After some algebra one finds that

$$
\begin{array}{rr}
\Gamma_{t t}^{t}=-\dot{\sigma}(t) & \Gamma_{t \mu}^{t}=0 \\
K_{t}^{t}=-e^{2 \sigma(t)}\left(\ddot{\sigma}(t)+\frac{\dot{\sigma}^{2}(t)}{2}\right) & K_{\mu}^{v}=-\frac{1}{2} e^{2 \sigma(t)} \dot{\sigma}^{2}(t) \delta_{\mu}{ }^{v} \\
K_{t t}=\left(\ddot{\sigma}(t)+\frac{\dot{\sigma}^{2}(t)}{2}\right) & K_{\mu v}=-\frac{e^{2 \sigma(t)}}{2} \dot{\sigma}^{2}(t) g_{\mu v}
\end{array}
$$

where $\dot{\sigma}(t)$ is the notation for

$$
\dot{\sigma}(t)=\frac{\mathrm{d} \sigma(t)}{\mathrm{d} t}
$$

In particular this implies that

$$
K_{\mathrm{L}}^{\mathrm{L}}=-e^{2 \sigma(t)}\left(\frac{d+1}{2} \dot{\sigma}^{2}(t)+\ddot{\sigma}(t)\right) .
$$

It then easily follows that

$$
\begin{aligned}
& \overline{\operatorname{Ric}}_{t t}=-d\left(\ddot{\sigma}(t)+\dot{\sigma}^{2}(t)\right) \\
& \overline{\operatorname{Ric}}_{t \mu}=0 \\
& \overline{\operatorname{Ric}}_{\mu \nu}=R i c_{\mu \nu}+\bar{g}_{\mu \nu}\left(d \dot{\sigma}^{2}(t)+\ddot{\sigma}(t)\right) .
\end{aligned}
$$

The other terms in the equations of motion read

$$
\begin{aligned}
\bar{H}_{\mu \nu}^{2} & =H_{\mu \alpha \beta} H_{v \gamma \delta} \bar{g}^{\alpha \gamma} \bar{g}^{\beta \delta}=e^{-4 \sigma(t)} H_{\mu \nu}^{2} \\
\bar{\nabla}_{\mathrm{M}} \bar{\nabla}_{\mathrm{N}} \Phi & =\partial_{\mathrm{M}} \partial_{\mathrm{N}} \Phi-\bar{\Gamma}_{\mathrm{MN}}^{\lambda} \partial_{\lambda} \Phi
\end{aligned}
$$

now, $\bar{\Gamma}_{\mu \nu}^{t}=-\dot{\sigma}(t) \bar{g}_{\mu \nu}$ so

$$
\begin{aligned}
\bar{\nabla}_{t} \bar{\nabla}_{t} \Phi & =\ddot{\Phi}(t) \\
\bar{\nabla}_{\mu} \bar{\nabla}_{t} \Phi & =0 \\
\bar{\nabla}_{\mu} \bar{\nabla}_{\nu} \Phi & =\dot{\sigma}(t) \dot{\Phi}(t) \bar{g}_{\mu v} .
\end{aligned}
$$

These are all the ingredients we need to write the equations of motion:

$$
\overline{\operatorname{Ric}}_{\mathrm{MN}}-\frac{1}{4} \bar{H}_{\mathrm{MN}}^{2}+2 \bar{\nabla}_{\mathrm{M}} \bar{\nabla}_{\mathrm{N}} \Phi=0 .
$$

Splitting the time component we obtain

$$
\begin{aligned}
& \operatorname{Ric}_{t t}+2 \partial_{t} \partial_{t} \Phi(t)=-d\left(\ddot{\sigma}(t)+\dot{\sigma}^{2}(t)\right)+2 \ddot{\Phi}(t)=0 \\
& \operatorname{Ric}_{\mu \nu}\left(1-e^{-4 \sigma(t)}\right)+\bar{g}_{\mu \nu}\left(d \dot{\sigma}^{2}(t)+\ddot{\sigma}(t)-2 \dot{\sigma}(t) \dot{\Phi}(t)\right)=0
\end{aligned}
$$


where we have used the equations of motion for the system in $\sigma=0$ :

$$
\text { Ric }_{\mu \nu}=\frac{1}{4} H_{\mu v}^{2}
$$

The system admits a solution if and only if $g_{\mu \nu}$ is Einstein (since the original system didn't have any dilaton). Taking the trace with $\bar{g}^{\mathrm{MN}}$ we obtain the system:

$$
\left\{\begin{array}{l}
d\left(\ddot{\sigma}(t)+\dot{\sigma}^{2}(t)\right)-2 \ddot{\Phi}(t)=0 \\
\operatorname{Re} e^{-2 \sigma(t)}\left(1-e^{-4 \sigma(t)}\right)+d\left(d \dot{\sigma}^{2}(t)+\ddot{\sigma}(t)-2 \dot{\sigma}(t) \dot{\Phi}(t)\right)=0
\end{array}\right.
$$

Introducing

$$
Q(t)=-\dot{\Phi}(t)+\frac{d}{2} \dot{\sigma}(t)
$$

the equations become:

$$
\left\{\begin{array}{l}
\dot{Q}(t)=-d \dot{\sigma}^{2}(t) \\
\ddot{\sigma}(t)=-\frac{R}{d} e^{-2 \sigma(t)}\left(1-e^{-4 \sigma(t)}\right)-2 \dot{\sigma}(t) Q(t)
\end{array}\right.
$$

This second equation has precisely the structure of the motion in a potential

$$
V(\sigma)-V(0)=\frac{R}{6 d} e^{-6 \sigma}\left(1-3 e^{4 \sigma}\right) \sim-\frac{R}{3 d}+\frac{2 R}{d} \sigma^{2} .
$$

and with a time-dependent friction coefficient $Q(t)$. In the limit of $Q \rightarrow \infty$ we clearly recover Eq. (6.63) with the same potential $V(\sigma)$ when identifying the energy scale $\mu$ for the off-shell system with the time direction here following

$$
\log \mu=\frac{\pi \bar{Q}}{\lambda} t
$$

Linearization. The system (6.83) can be solved numerically and typical results for large $Q(0)$ and small $Q(0)$ are presented in Fig. 6.4.

A further step can be made by linearization. Introduce

$$
\Sigma(t)=\dot{\sigma}(t)
$$

the system becomes a first order one:

$$
\left\{\begin{array}{l}
\dot{Q}(t)=-d \Sigma^{2}(t) \\
\dot{\sigma}(t)=\Sigma(t) \\
\dot{\Sigma}(t)=-V^{\prime}(\sigma(t))-2 \Sigma(t) Q(t)
\end{array}\right.
$$

which has a fixed point for $(Q, \sigma, \Sigma)=(\bar{Q}, 0,0)$ where $\bar{Q}$ is a constant. Around this point the equations read:

$$
\left\{\begin{array}{l}
\dot{Q}(t)=0 \\
\dot{\sigma}(t)=\Sigma(t) \\
\dot{\Sigma}(t)=-V^{\prime \prime}(0) \sigma(t)-2 \bar{Q} \Sigma(t)=-\frac{4 R}{d} \sigma(t)-2 \bar{Q} \Sigma(t)
\end{array}\right.
$$




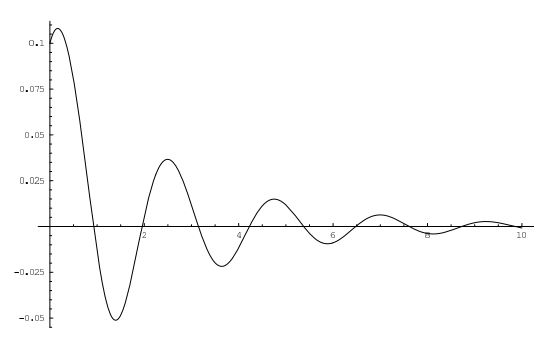

(a) Small $Q(0)$

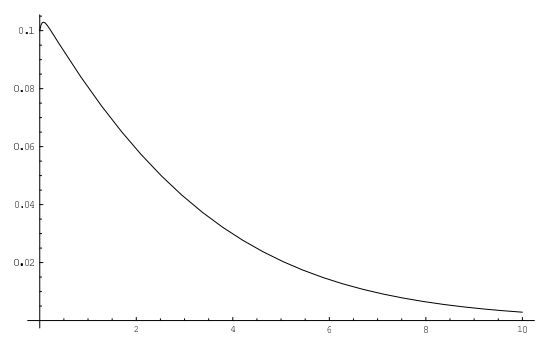

(b) Large $Q(0)$

Figure 6.4: Typical behaviour for $\sigma(t)$ in the system (6.83) for (a) small and (b) large (positive) initial values of $Q(t)$.

so, $Q$ decouples (and remains constant) and the equation of motion around the fixed point is

$$
\frac{\mathrm{d}^{2} \sigma(t)}{\mathrm{d} t^{2}}=-\frac{4 R}{d} \sigma(t)-2 \bar{Q} \frac{\mathrm{d} \sigma(t)}{\mathrm{d} t}
$$

which can be integrated giving

$$
\sigma(t)=C_{1} \exp \left[-\left(\bar{Q}+\sqrt{\bar{Q}^{2}-\frac{4 R}{d}}\right) t\right]+C_{2} \exp \left[-\left(\bar{Q}-\sqrt{\bar{Q}^{2}-\frac{4 R}{d}}\right) t\right]
$$

with $C_{1}$ and $C_{2}$ integration constants.

For positive $\bar{Q}$ the solution converges to $\sigma=0$ with or without oscillations if $\bar{Q}^{2} \lessgtr 4 R / d$. In terms of $\sigma(t)$ and $\Phi(t)$ this limit solution is

$$
\sigma(t) \underset{t \rightarrow \infty}{\longrightarrow} 0 \quad \Phi(t) \sim-\bar{Q} t,
$$

which is not surprisingly the initial conformal model in Eq. (6.57) plus a linear dilaton.

The meaning of $\bar{Q} . \quad \bar{Q}$ is linked to the dilaton: larger values correspond to negative and larger absolute values for $\Phi$, i.e. moving further inside the perturbative regime. On the other hand, negative values of $\bar{Q}$ give diverging solutions, but in this case the dilaton grows (see Eq. 6.82) and the very underlying perturbative approach collapses. It is worth to remark that if we make an hypothesis of uniqueness for the system (6.83), $Q$ can't change sign because $Q(t)=0, \sigma(t)=1$ is a solution (the starting conformal model with constant dilaton).

A better understanding of the actual meaning of this parameter can be obtained if we consider the limiting situation of linear dilaton. In this case, in fact, it is immediate to derive the central-charge of the overall system:

$$
c=(d+1)-3 \bar{Q}^{2}-c_{d}+c_{I}=0,
$$


where $c_{d}$ is the central-charge of the conformal system in Eq. (6.57) (e.g. 6/ $(k+2)$ for the $S U(2)$ WZW model) and $c_{I}$ is the internal central-charge. If follows that for a critical model

$$
\bar{Q}^{2}=\frac{1}{3}\left(d+1-c_{d}+c_{I}\right)
$$

and $\bar{Q}$ is essentially a measure of the deficit.

A final remark regards the consistency of the approximation for the dynamics one obtains from the RG-flow equation (6.63), corresponding to a $Q \rightarrow$ $\infty$ limit. The linearized system (6.88) provides a justification for such limit: in fact the time scale for $Q(t)$ is comparably larger than $\sigma(t)^{\prime} \mathrm{s}$ - to the point that the former decouples around the fixed point. For this reason it can be taken as a constant (fixed by the initial conditions) if we just concentrate on the evolution of the warping factor $\sigma(t)$.

\subsection{Cosmological interpretation}

The type of backgrounds we are studying are time-dependent and as such can have a cosmological interest. For this reason, since there is a non-trivial dilaton, one should better move to the Einstein frame (as opposed to the string frame we've been using thus far). This means that the metric is written as:

$$
\tilde{g}_{\mathrm{MN}}=e^{-\Phi(t) / 2} \bar{g}_{\mathrm{MN}}
$$

and after a coordinate change

$$
\tau(t)=\int e^{-\Phi(t) / 4} \mathrm{~d} t
$$

can be put back to the same warped product form as in Eq. (6.66):

$$
\begin{array}{r}
\widetilde{\mathrm{d} s^{2}}=\tilde{g}_{\mathrm{MN}} \mathrm{d} x^{\mathrm{M}} \mathrm{d} x^{\mathrm{N}}=-\mathrm{d} \tau^{2}+\left.e^{2 \sigma(t)-\Phi(t) / 2}\right|_{t=t(\tau)}\left(g_{\mu \nu} \mathrm{d} x^{\mu} \mathrm{d} x^{\nu}\right)= \\
=-\mathrm{d} \tau^{2}+w(\tau)\left(g_{\mu \nu} \mathrm{d} x^{\mu} \mathrm{d} x^{\nu}\right) .
\end{array}
$$

Cosmologically interesting solutions are obtained when $d=3$. In this case the $H$ field is proportional to the volume form on $g$. This implies that $H_{\mu \nu}^{2} \propto g_{\mu v}$ and then the equations reduce to

$$
\operatorname{Ric}_{\mu \nu}=\Lambda^{2} g_{\mu \nu}
$$

ie $g_{\mu v}$ is to be the metric of an Einstein three-manifold (the most simple case being a three-sphere). What we get then is a typical example of FriedmannRobertson-Walker (FRW) spacetime such as those already studied in [Tse92b, Tse92a, GP94, CLW94]. As such it describes the time evolution of an isotropic spacetime (or more in general of a spacetime with the symmetries of the conformal theory in Eq. (6.57)). Some intuition about the time evolution can be developed if we take the linearized system in Eq. (6.88) and consider the large 
$t$ limit. In fact, as remarked above the solution asymptotically approaches a linear dilaton background (which was already studied from this point of view in [ABEN89]):

$$
\sigma(t) \underset{t \rightarrow \infty}{\longrightarrow} 0 \quad Q(t)=\bar{Q} \quad \Phi(t) \sim-\bar{Q} t
$$

hence one verifies that the metric in the Einstein frame is asymptotically

$$
\widetilde{\mathrm{d} s^{2}} \sim-\mathrm{d} \tau^{2}+\bar{Q}^{2} \tau^{2}\left(g_{\mu \nu} \mathrm{d} x^{\mu} \mathrm{d} x^{\nu}\right)
$$

which corresponds to an expanding universe with curvature

$$
\tilde{R} \sim \frac{R+\bar{Q}^{2} d(d-1)}{\bar{Q}^{2} \tau^{2}}
$$

A similar result, with a polynomial expansion is found if we consider an exponential decrease for $\sigma(t)$, or better for $c(t)$ (in the linear limit $c(t)-1$ obeys the same equations as $\sigma(t))$. After a redefinition of the variables we can let

$$
c(t)=e^{-t}+1 \text {. }
$$

It is easy to check that in general ${ }^{5}$

$$
\tau(t)=\int c(t)^{-d / 16} e^{1 / 4 \int Q\left(t^{\prime}\right) \mathrm{d} t^{\prime}} \mathrm{d} t
$$

and in this linearized approximation the latter becomes

$$
\tau(t)=\int\left(e^{-t}+1\right)^{-d / 16} e^{\bar{Q} t / 4} \mathrm{~d} t .
$$

This integral can be solved analytically:

$\tau(u)=\frac{16}{d+4 \bar{Q}}\left(1+\frac{1}{u}\right)^{-d / 16} u^{\bar{Q} / 4}(1+u)^{d / 16}{ }_{2} F_{1}\left(\frac{d}{16}, \frac{d+4 \bar{Q}}{16} ; \frac{d+4 \bar{Q}}{16}+1,-u\right)$,

where $u=e^{t}$ and ${ }_{2} F_{1}$ is an hypergeometric function ${ }^{6}$. It is better however to consider the asymptotic behaviours. For $u \rightarrow \infty$ one finds that $\tau(u)$ and the warping factor $w(u)$ go like:

$$
\tau(u) \sim \frac{4}{\bar{Q}} u^{\bar{Q} / 4}, \quad w(u) \sim u^{\bar{Q} / 2},
$$

\footnotetext{
${ }^{5}$ On a side note, since $c(t)>0$ by construction the relation $\tau=\tau(t)$ is always invertible.

${ }^{6}$ The hypergeometric function ${ }_{2} F_{1}$ is defined as follows:

$$
{ }_{2} F_{1}(a, b ; c, u)=\sum_{k=0}^{\infty} \frac{(a)_{k}(b)_{k}}{(c)_{k}} \frac{z^{k}}{k !}
$$

where $(a)_{k}$ is the Pochhammer symbol

$$
(a)_{k}=\frac{\Gamma(a+k)}{\Gamma(a)}
$$


and consistently with the results above for the linear dilaton case (which is precisely the large- $u$ limit):

$$
w(\tau) \sim \tau^{2}
$$

similarly for small $u$ :

$$
\tau(u) \sim \frac{16}{d+4 \bar{Q}} u^{(d+4 \bar{Q}) / 16}, \quad \quad w(u) \sim u^{d / 4+2+\bar{Q} / 2}
$$

and then

$$
w(\tau) \sim \tau^{4+8(4-\bar{Q}) /(d+4 \bar{Q})} .
$$

Note that this behaviour precisely measures the effect of a finite value for $\bar{Q}$ and in fact for $\bar{Q} \rightarrow \infty$ we recover again $w(\tau) \sim \tau^{2}$. Summarizing, just as advertised, we get again a polynomially expanding universe (a so-called bigbang solution).

The analysis for the small- $\bar{Q}$ regime is clearly more difficult to be carried out analytically. Apart from numerical solutions (see Fig. 6.5), in general we can study $w(\tau)$ as a parametric curve in the $(w, \tau)$ plane defined by $(w(t), \tau(t))$. Then $\tau(t)$ appears to be a monotonically increasing function since $c(t)>0$ which implies that $w(\tau)$ has an extremum for each extremum in $w(t)$. This means that we expect the superposition of a polynomial expansion and a damped oscillation. The limiting situation is obtained when $\bar{Q}$ is small (but not vanishing), and for large $t, \tau(t) \sim t$ so that $w(\tau)$ slowly converges, oscillating, to a constant value. 


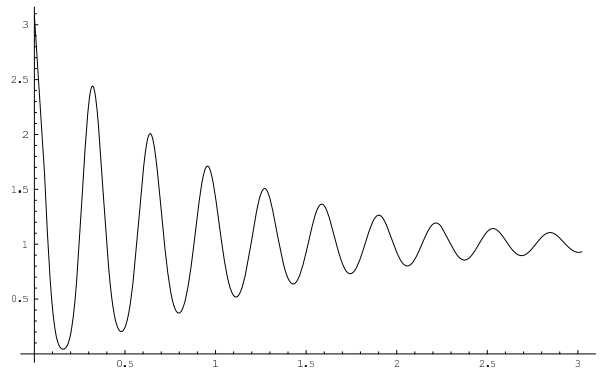

(a) $\bar{Q} \sim 0$

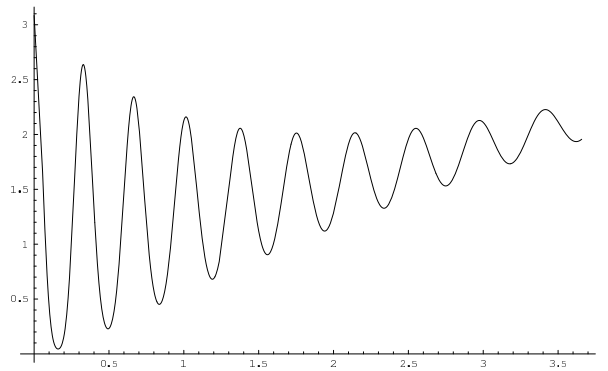

(b) Small $\bar{Q}$

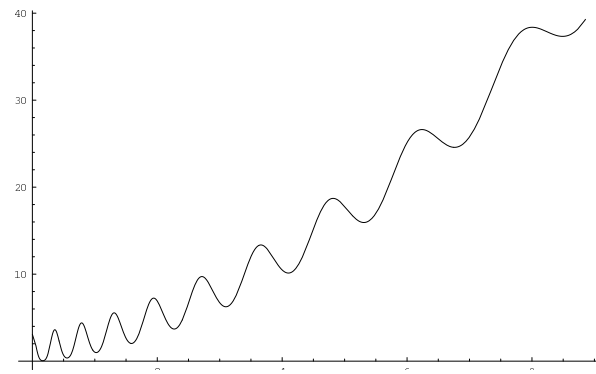

(c) Medium $\bar{Q}$

Figure 6.5: Typical behaviour for the warping factor in the small- $\bar{Q}$ regime. We consider (a) $\bar{Q}$ very small but not vanishing, (b) small $\bar{Q}$ and (c) larger $\bar{Q}$ (but still compatible with oscillations). 



\section{CHAPTER 7}

\section{Hyperbolic Spaces}

In this chapter we investigate type II and M-theory geometries written as direct products of constant-curvature spaces. We find in particular a class of backgrounds with hyperbolic components and we study their stability with respect to small fluctuations.

THIS CHAPTER does in some sense deviate from the main theme we devel1 oped in this thesis. In fact we will deal with type II theories in presence of Ramond-Ramond fields, that - to this moment - still elude a precise CFT treatment. For this reason our analysis will be mostly confined to supergravity considerations. On the other hand we still continue to follow one of the main guiding threads, i.e. look for backgrounds with simple geometric interpretation, which in this case means (maximally) symmetric spaces, with special emphasis on hyperbolic, negative curvature, Poincaré spaces. We will show in fact that these spaces can be used as building blocks for M-theory and type II backgrounds, in genuinely perturbative configurations or in presence of $\mathrm{D}$ branes, both in the non-compact and in the compact part after discrete identifications. In particular we find a series of M-theory solutions that can be obtained starting with the usual $\mathrm{AdS}_{7} \times S^{4}$ by splitting the anti-de Sitter in a AdS $\times H$ product and verify their stability with respect to small fluctuations. Finally we also show how obtaining negative-curvature Euclidean signature spaces is not in general an easy task and in particular show that the presence of orientifold planes, giving a negative contribution to the stress-energy tensor is not enough to allow for $\mathrm{H}_{3}$ spaces in type IIB theories.

\subsection{M-theory solutions}

Let us start with the action of the bosonic sector of eleven-dimensional supergravity:

$$
S=\frac{1}{2 \kappa_{11}^{2}} \int \mathrm{d}^{11} x \sqrt{-g}\left(R-\frac{1}{2}\left|F_{[4]}\right|^{2}\right)+\frac{1}{12 \kappa_{11}^{2}} \int A_{[3]} \wedge F_{[4]} \wedge F_{[4]},
$$

and the corresponding equations of motion

$$
R_{\mu \nu}-\frac{1}{2} R g_{\mu v}-\frac{1}{2}\left|F_{[4]}^{2}\right|_{\mu \nu}+\frac{1}{4}\left|F_{[4]}^{2}\right| g_{\mu \nu}=0,
$$


where with the notation $\left|F_{[n]}\right|^{2}$ we mean

$$
\left|F_{[p]}\right|^{2}=\frac{1}{p !} F_{\mu_{1} \mu_{2} \ldots \mu_{p}} F^{\mu_{1} \mu_{2} \ldots \mu_{p}}
$$

The ansatz we want to study is the following. We consider direct products of symmetric spaces of the form $M_{11}=M_{0} \times M_{1} \times M_{2} \times \ldots$ where $M_{0}$ has signature $-,+, \ldots,+$ and all the other spaces are Riemann. Since we assume that they are all symmetric spaces, we can split the Ricci tensor in blocks and each block will be proportional to the metric of the corresponding submanifold. To fix the notation we can introduce the parameters $\Lambda_{i}$ as

$$
\left.R_{\mu v}\right|_{i}=\left.\Lambda_{i} g_{\mu v}\right|_{i}
$$

so that the Ricci scalars are given by

$$
R_{i}=\Lambda_{i} \operatorname{dim} M_{i}=\Lambda_{i} d_{i}
$$

In particular we can raise an index and rewrite

$$
\left.R^{\mu}{ }_{v}\right|_{i}=\left.\Lambda_{i} \delta^{\mu}{ }_{v}\right|_{i}
$$

The Poincare invariance constraint fixes the allowed gauge fields to be proportional to the volume form of each submanifold. It is always possible to perform an electric/magnetic duality so that there's no field on the Minkowskian submanifold. This means that we can consider gauge fields having the form:

$$
F_{\left[d_{i}\right]}=F_{i}=Q_{i} \tilde{\omega}_{i}
$$

where $F_{i}$ is a $d_{i}$-form, $Q_{i} \in \mathbb{N}$ and $\tilde{\omega}_{i}=\widetilde{\omega_{M_{i}}}$ is the volume form on $M_{i}$, normalized to one. It is useful to rewrite the expression above as

$$
F_{i}=\sqrt{2} k_{i} Q_{i}\left|\Lambda_{i}\right|^{d_{i} / 2} \omega_{i}
$$

where $\omega_{i}$ is the volume form on $M_{i}$ and $k_{i}$ is a constant whose value is

- $k_{i}=\Gamma(i / 2) /\left(2 \sqrt{2} \pi^{i / 2}\right)$ for a sphere $S^{i}$

- $k_{i}=1 /(4 \pi(g-1))$ for a genus $g$ Riemann surface $H^{2} / \Gamma$

- some value that completely identifies the lattice in a $H^{3} / \Gamma$ compactification (rigidity theorem for three-manifolds [Thurston]).

In coordinates

$$
\left.F_{i}\right|_{\mu_{1} \ldots \mu_{d_{i}}}=k_{i} Q_{i} \Lambda_{i}^{d_{i} / 2} \sqrt{2 \operatorname{det} g_{i}} \epsilon_{\mu_{1} \ldots \mu_{d_{i}}}
$$

which implies (as one can verify in a non-coordinate basis):

$$
\left.\left.F_{i}\right|_{\mu \mu_{2} \ldots \mu_{d_{i}}} F_{i}\right|^{v \mu_{2} \ldots \mu_{d_{i}}}=2(n-1) ! k_{i}^{2} Q_{i}^{2} \Lambda^{d_{i}} \delta^{\mu}{ }_{v}
$$


Furthermore

$$
\begin{aligned}
\left|F_{i}^{2}\right|^{\mu}{ }_{v} & =2 k_{i}^{2} Q_{i}^{2}\left|\Lambda_{i}\right|^{d_{i}} \delta^{\mu}{ }_{v} \\
\left|F_{i}^{2}\right| & =2 k_{i}^{2} Q_{i}^{2}\left|\Lambda_{i}\right|^{d_{i}} .
\end{aligned}
$$

We are now in position to write the equations of motion that will simply translate into an algebraic system for the $\left(\Lambda_{0}, \Lambda_{i}\right)$ :

$$
\left\{\begin{array}{l}
\Lambda_{0}-\frac{1}{2} R=-\frac{1}{2} \sum_{j} k_{j}^{2} Q_{j}^{2}\left|\Lambda_{j}\right|^{d_{j}}, \\
\Lambda_{i}-\frac{1}{2} R=-\frac{1}{2} \sum_{j}(-)^{\delta_{i j}} k_{j}^{2} Q_{j}^{2}\left|\Lambda_{j}\right|^{d_{j}},
\end{array}\right.
$$

where

$$
R=d_{0} \Lambda_{0}+\sum_{i} d_{i} \Lambda_{i}
$$

Let us now turn to study some particular examples.

$M^{1,6-d} \times M^{d} \times M^{4}$

Let us consider as an example the case $M_{11}=M_{0} \times M_{1} \times M_{2}$ where $\left(d_{0}, d_{1}, d_{2}\right)=$ $(7-d, d, 4)$. If we turn $F_{2}$ in the equations of motion read:

$$
\left\{\begin{array}{l}
\Lambda_{0}-\frac{1}{2} R=-\frac{1}{2} k_{2}^{2} Q_{2}^{2} \Lambda_{2}^{4} \\
\Lambda_{1}-\frac{1}{2} R=-\frac{1}{2} k_{2}^{2} Q_{2}^{2} \Lambda_{2}^{4} \\
\Lambda_{2}-\frac{1}{2} R=\frac{1}{2} k_{2}^{2} Q_{2}^{2} \Lambda_{2}^{4}
\end{array}\right.
$$

with $R=(7-d) \Lambda_{0}+d \Lambda_{1}+4 \Lambda_{2}$. The solution is:

$$
\Lambda_{0}=\Lambda_{1}=-\frac{1}{2}\left(\frac{3}{2}\right)^{1 / 3} \frac{1}{\left(k_{2} Q_{2}\right)^{2 / 3}}, \quad \Lambda_{2}=\left(\frac{3}{2}\right)^{1 / 3} \frac{1}{\left(k_{2} Q_{2}\right)^{2 / 3}}
$$

and given the curvatures, this describes an $\mathrm{AdS}_{7-d} \times H_{d} \times S^{4}$ space.

A few remarks are in order. First of all, the result doesn't depend on $d$ and in particular it would be the same for $d=0$ (which is some sort of limit case). In other words, at the level of the equations of motion, we can't distinguish between an $\mathrm{AdS}_{7}$ space and any product of the form $\mathrm{AdS}_{7-d} \times H_{d}$ once the respective curvatures are such as

$$
\frac{R_{\mathrm{AdS}}}{7-d}=\frac{R_{H}}{d}
$$

This calculation is generalizable to any product of the form $M_{0} \times M_{1} \times \ldots \times$ $M_{n}$ with dimensions $\left(d_{0}, d_{1}, \ldots, d_{n-1}, 4\right)$. In fact the equations of motion read

$$
\left\{\begin{array}{l}
\Lambda_{0}-\frac{1}{2} R=-\frac{1}{2} k_{n}^{2} Q_{n}^{2} \Lambda_{n}^{4}, \\
\Lambda_{1}-\frac{1}{2} R=-\frac{1}{2} k_{n}^{2} Q_{n}^{2} \Lambda_{n}^{4}, \\
\cdots \\
\Lambda_{n-1}-\frac{1}{2} R=-\frac{1}{2} k_{n}^{2} Q_{n}^{2} \Lambda_{n}^{4}, \\
\Lambda_{n}-\frac{1}{2} R=\frac{1}{2} k_{2}^{2} Q_{2}^{2} \Lambda_{2}^{4},
\end{array}\right.
$$


with $R=d_{0} \Lambda_{0}+d_{1} \Lambda_{1}+\ldots+d_{n} \Lambda_{n}$. From the system above we conclude that $\Lambda_{0}=\Lambda_{1}=\ldots=\Lambda_{n-1}$ and then we are back to the situation above:

$\Lambda_{0}=\Lambda_{1}=\ldots=\Lambda_{n-1}=-\left(\frac{3}{2}\right)^{1 / 3} \frac{1}{2\left(k_{n} Q_{n}\right)^{2 / 3}}, \quad \Lambda_{n}=\left(\frac{3}{2}\right)^{1 / 3} \frac{1}{\left(k_{n} Q_{n}\right)^{2 / 3}}$.

We have thus found a series of possible M-theory backgrounds where the antide Sitter component is split into two or more subspaces of the form

$$
\mathrm{AdS}_{n} \rightarrow \mathrm{AdS}_{n-p} \times H_{p}
$$

with Ricci scalars obeying

$$
\frac{R^{(n)}}{n}=\frac{R^{(n-p)}}{n-p}=\frac{R^{(p)}}{p} .
$$

In particular we get the direct products $\mathrm{AdS}_{2} \times \mathrm{H}_{2} \times \mathrm{H}_{3} \times \mathrm{S}^{4}, \mathrm{AdS}_{2} \times \mathrm{H}_{5} \times \mathrm{S}^{4}$, $\mathrm{AdS}_{3} \times H_{2} \times H_{2} \times S^{4}, \mathrm{AdS}_{3} \times H_{4} \times S^{4}, \mathrm{AdS}_{4} \times H_{3} \times S^{4}, \mathrm{AdS}_{5} \times H_{2} \times S^{4}$.

$M^{1,3} \times M^{d} \times M^{7-d}$

The dual situation is obtained for $\left(d_{0}, d_{1}, d_{2}\right)=(4, d, 7-d)$. In this case we can turn on the 7-form field

$$
F_{[7]}=\sqrt{2} k_{0} Q_{0} \Lambda_{1}^{d / 2} \Lambda_{2}^{(7-d) / 2}
$$

and the equations of motion read:

$$
\left\{\begin{array}{l}
\Lambda_{0}-\frac{1}{2} R=-\frac{1}{2} k_{0}^{2} Q_{0}^{2}\left|\Lambda_{1}^{d} \Lambda_{2}^{7-d}\right|, \\
\Lambda_{1}-\frac{1}{2} R=\frac{1}{2} k_{0}^{2} Q_{0}^{2}\left|\Lambda_{1}^{d} \Lambda_{2}^{7-d}\right|, \\
\Lambda_{2}-\frac{1}{2} R=\frac{1}{2} k_{0}^{2} Q_{0}^{2}\left|\Lambda_{1}^{d} \Lambda_{2}^{7-d}\right|,
\end{array}\right.
$$

with $R=4 \Lambda_{0}+d \Lambda_{1}+(7-d) \Lambda_{2}$. Again the solution is easily found

$$
\Lambda_{0}=-2 \frac{3^{1 / 6}}{\left(k_{0} Q_{0}\right)^{2 / 3}} \quad \Lambda_{1}=\Lambda_{2}=\frac{3^{1 / 6}}{\left(k_{0} Q_{0}\right)^{2 / 3}} .
$$

Just as before this does not depend on $d$ and shows that at this level an $\mathrm{AdS}_{4} \times$ $S^{d} \times S^{7-d}$ space is not distinguishable from a $\mathrm{AdS}_{4} \times S^{7}$ one. Again we can consider more general configurations with dimensions $\left(4, d_{1}, \ldots, d_{n}\right)$ to find that the solution remains the same

$$
\Lambda_{0}=-2 \frac{3^{1 / 6}}{\left(k_{0} Q_{0}\right)^{2 / 3}}, \quad \Lambda_{1}=\Lambda_{2}=\ldots=\Lambda_{n}=\frac{3^{1 / 6}}{\left(k_{0} Q_{0}\right)^{2 / 3}} .
$$

This describes a second series of solutions in which a sphere is split into the product of two smaller spheres according to

$$
S^{n} \rightarrow S^{n-p} \times S^{p}
$$


with Ricci scalars obeying as above

$$
\frac{R^{(n)}}{n}=\frac{R^{(n-p)}}{n-p}=\frac{R^{(p)}}{p},
$$

and then we recover $\mathrm{AdS}_{4} \times S^{2} \times S^{2} \times S^{3}, \mathrm{AdS}_{4} \times S^{2} \times S^{5}, \mathrm{AdS}_{4} \times S^{3} \times S^{2} \times S^{2}$, $\mathrm{AdS}_{4} \times S^{3} \times S^{4}, \mathrm{AdS}_{4} \times S^{5} \times S^{2}$.

Finally we can combine the two types of splitting and put $\mathrm{AdS}_{2} \times \mathrm{H}^{2}$ instead of $\mathrm{AdS}_{4}$ and $S^{2} \times S^{2}$ instead of $S^{4}$ in the former series.

The key ingredient to these constructions is the fact that with a careful choice of radii the product spaces still remain Einstein and this is all one needs to satisfy the equations of motion. This means of course that in general one can use any Einstein manifold with the proper curvature. In particular, then, instead of the five-sphere $S^{5}$ one can put a generalization of the $S^{3} \times S^{2}$ product, such as any of the representatives of the two-parameter class of spaces $T^{p, q} \mathrm{ob}-$ tained as $S^{1}$ fibrations over $S^{2} \times S^{2}$ or equivalently as the coset $(S U(2) \times S U(2)) / U(1)$, the parameter being the embedding indexes of $U(1)$ in the $S U(2)^{\prime} s^{1}$. Similarly, instead of $\mathrm{AdS}^{5}$ one can put a space written as a time-fibration over $\mathrm{H}_{2} \times \mathrm{H}_{2}$ or as the coset $(S L(2, \mathbb{R}) \times S L(2, \mathbb{R})) / \mathbb{R}$. The metric of such $L_{Q_{1}, Q_{2}}$ space can be written as

$$
\begin{array}{r}
\mathrm{d} s^{2}=Q_{1}\left(\frac{\mathrm{d} x^{2}}{1+x^{2}}+\left(1+x^{2}\right) \mathrm{d} u^{2}\right)+Q_{2}\left(\frac{\mathrm{d} y^{2}}{1+y^{2}}+\left(1+y^{2}\right) \mathrm{d} v^{2}\right)+ \\
+\frac{2 Q_{1}\left(Q_{1}-2 Q_{2}\right)}{Q_{2}}\left(\mathrm{~d} t+x \mathrm{~d} u+\frac{Q_{2}}{Q_{1}} \sqrt{\left.\frac{Q_{2}-2 Q_{1}}{Q_{1}-2 Q_{2}} y \mathrm{~d} v\right)^{2} .}\right.
\end{array}
$$

Such cosets were studied in [PZT00] where they were found to be exact string backgrounds by using a construction very close to the asymmetric cosets of Ch. 3.

Of course since in general these geometries don't preserve any supersymmetry we should address the problem of their stability, which we do in the following section.

\subsection{Stability}

The set of solutions we found above are not in general protected by supersymmetry. This implies in particular that we should care about their stability. In our analysis we will deal with the breathing modes of the compact $H_{n}$ and $S^{n}$ internal manifolds which, in an effective action, are to be described by scalar fields. The stability (with respect to small fluctuations) will then translate intro the positivity of the squared mass for such fields, condition that can be relaxed into the respect of a Breitenlohner-Freedman bound when the spacetime is of the anti-de Sitter type.

\footnotetext{
${ }^{1}$ This clearly is the basis of the conifold.
} 
First of all then we give an explicit derivation for the bound, so to completely fix the notation and then construct the general expression for the spacetime effective action for the breathing modes - hence finding again the same solutions as above as stationary points for a potential whose Hessian matrix encodes the stability for the background.

\section{Breitenlohner-Freedman bound}

Anti-de Sitter Consider an action of the kind

$$
S=\int \mathrm{d}^{d} x \sqrt{-g^{d}}\left(R-\frac{1}{2} \partial_{\mu} \phi \partial^{\mu} \phi-V(\phi)\right)
$$

the equation of motion for $\phi$ reads

$$
-\nabla_{\mu} \partial^{\mu} \phi=-\frac{\partial V}{\partial \phi}
$$

The relations that we obtain are all tensorial so we can just choose a suitable coordinate system, knowing that the result will remain invariant. In $\mathrm{AdS}_{d}$ a good choice would be

$$
\mathrm{d} s^{2}=\mathrm{d} r^{2}+e^{2 H r}\left(-\mathrm{d} t^{2}+\mathrm{d} x_{1}^{2}+\cdots+\mathrm{d} x_{d-2}^{2}\right)
$$

and we can consider a potential $V$ with a minimum in $\phi=0$ :

$$
V(\phi)=V_{0}+\frac{m^{2}}{2} \phi^{2}
$$

The equation of motion for an $r$-dependent field $\phi$ reads

$$
\phi^{\prime \prime}+(d-1) H \phi^{\prime}-m^{2} \phi=0 .
$$

Solving it one can see that the presence of the friction term effectively changes the mass to

$$
M^{2}=\left(\frac{d-1}{2} H\right)^{2}+m^{2} \geq 0
$$

or, given that $R=-d(d-1) H^{2}$

$$
M^{2}=-\frac{d-1}{4 d} R+m^{2} \geq 0
$$

or, again, in terms of minimum of the potential:

$$
M^{2}=-\frac{d-1}{4(d-2)}\langle V\rangle+m^{2} \geq 0 .
$$

Positivity of the effective mass squared, and thus stability, translate therefore into a less stringent constraint for $\mathrm{m}^{2}$. This is the BF bound. 
$L_{Q_{1}, Q_{2}}$ spaces. The presence of the bound is due to the curvature of the manifold. It can be restated by saying that in an appropriate coordinate system the Klein-Gordon equation can be put in the form

$$
-\square \phi+m^{2} \phi=(-\tilde{\square}+\Delta) \phi+m^{2} \phi=-\tilde{\square} \phi+M^{2} \phi=0,
$$

where $\square$ is the d'Alembertian for the curved space, $\square$ is the d'Alembertian for flat Minkowski space and $\Delta$ is some constant depending on the curvature and other details of the geometry $\left(\Delta=-(d-1) /(4 d) R\right.$ in the case of $\left.\operatorname{AdS}_{d}\right)$. It is natural to expect a similar behaviour for other negative-curvature spaces, but the precise value of $\Delta$ will depend on the details. In particular it is interesting to consider the $L_{Q, 1, Q_{2}}$ spaces introduced above. Again, as before, we can choose a coordinate system and then use the fact that the equations we get are tensor relations and hence invariant. Take into example the following metric:

$$
\begin{aligned}
\mathrm{d} s^{2}=Q_{1} & \left(\frac{\mathrm{d} x^{2}}{1+x^{2}}+\left(1+x^{2}\right) \mathrm{d} u^{2}\right)+Q_{2}\left(\frac{\mathrm{d} y^{2}}{1+y^{2}}+\left(1+y^{2}\right) \mathrm{d} v^{2}\right)+ \\
+ & \frac{2 Q_{1}\left(Q_{1}-2 Q_{2}\right)}{Q_{2}}\left(\mathrm{~d} t+x \mathrm{~d} u+\frac{Q_{2}}{Q_{1}} \sqrt{\left.\frac{Q_{2}-2 Q_{1}}{Q_{1}-2 Q_{2}} y \mathrm{~d} v\right)^{2},}\right.
\end{aligned}
$$

which describes an $L_{Q_{1}, Q_{2}}$ space with Ricci scalar

$$
R=-\frac{5}{3} \frac{Q_{1}+Q_{2}}{Q_{1} Q_{2}}
$$

The d'Alembertian on $\phi(x, y)$ gives:

$$
\square \phi(x, y)=\frac{1}{Q_{1}}\left[\left(1+x^{2}\right) \phi_{x x}+2 x \phi_{x}\right]+\frac{1}{Q_{2}}\left[\left(1+y^{2}\right) \phi_{y y}+2 y \phi_{y}\right] .
$$

This is the same expression we would have got by considering the d'Alembertian in an $\mathrm{AdS}_{3} \times \mathrm{AdS}_{3}$ space with coordinates

$$
\mathrm{d} s^{2}=\frac{1}{Q_{1}}\left[\frac{\mathrm{d} x^{2}}{1+x^{2}}-\mathrm{d} t^{2}-2 x \mathrm{~d} t \mathrm{~d} u+\mathrm{d} u^{2}\right]+\frac{1}{Q_{2}}\left[\frac{\mathrm{d} y^{2}}{1+y^{2}}-\mathrm{d} \tau^{2}-2 y \mathrm{~d} \tau \mathrm{d} v+\mathrm{d} v^{2}\right] .
$$

The two subspaces have curvature

$$
R_{i}=-\frac{3}{2 Q_{i}}
$$

and since the shifts compose linearly this gives an overall shift

$$
M^{2}=m^{2}+\frac{1}{4 Q_{1}}+\frac{1}{4 Q_{2}}=m^{2}+\frac{1}{4} \frac{Q_{1}+Q_{2}}{Q_{1} Q_{2}},
$$

which is therefore the BF bound for $L_{Q_{1}, Q_{2}}$ space. This can be compared with an $\mathrm{AdS}_{5}$ space with the same curvature as in Eq. (7.39), where the shift would be given by

$$
M^{2}=m^{2}+\frac{1}{3} \frac{Q_{1}+Q_{2}}{Q_{1} Q_{2}} .
$$


In this case the shift is larger: in some sense then, as one might have expected, an anti de-Sitter space is more stable with respect to small fluctuations than a $L_{Q_{1}, Q_{2}}$ space with the same scalar curvature.

\section{Effective low-dimensional description}

We want to write the $d$-dimensional effective action for a $\mathrm{AdS}_{d} \times M_{1} \times M_{2}$ background, where $M_{i}$ are constant curvature spaces, starting from the following sector of the M-theory action

$$
S=\frac{1}{2 \kappa_{11}^{2}} \int \mathrm{d}^{11} x \sqrt{-g^{(11)}}\left(R^{11}-V\right)
$$

where $V$ takes into account the presence of the fluxes. In order to study the stability of the product background at hand let us consider the following ansatz:

$$
\mathrm{d} s_{11}^{2}=\mathrm{d} s^{2}\left(M^{(d)}\right)+e^{2 \varphi_{1}(x)} \mathrm{d} s^{2}\left(M^{(1)}\right)+e^{2 \varphi_{2}(x)} \mathrm{d} s^{2}\left(M^{(2)}\right)
$$

where the fields $\varphi_{i}$ depend only on the coordinates of $M^{(d)}$. The strategy is the following:

(a) write the curvature $R^{(11)}$ in terms of the curvature for $M^{(d)}$

(b) separate the determinant $g^{(11)}$ in its compact and non-compact part

(c) Weyl-rescale the four-dimensional metric to get a canonical Einstein-Hilbert action

(d) rescale the scalar fields $\varphi_{i}$ to get a canonical kinetic term

(e) study the potential and verify the stability taking into account the BF bound.

Step a For a warped product

$$
\mathrm{d} s^{2}=\mathrm{d} s^{2}\left(M^{(d)}(x)\right)+e^{2 \varphi(x)} \mathrm{d} s^{2}\left(M^{(1)}\right)
$$

where $M^{(1)}$ has dimension $d_{1}$, the Ricci scalar is

$$
R=R^{(1)}+e^{-2 \varphi(x)} R^{(2)}-2 d_{1} \nabla_{\mu} \partial^{\mu} \varphi(x)+d_{1}\left(d_{1}-1\right) \partial_{\mu} \varphi(x) \partial^{\mu} \varphi(x)
$$

where the covariant derivative and the raising is done with the warped product metric. Note that the result only depends on $d_{1}$ and not on $d$. This easily generalizes to a warped product with three factors like the ones we study

$$
\mathrm{d} s^{2}=\mathrm{d} s^{2}\left(M^{(d)}(x)\right)+e^{2 \varphi_{1}(x)} \mathrm{d} s^{2}\left(M^{(1)}\right)+e^{2 \varphi_{2}(x)} \mathrm{d} s^{2}\left(M^{(2)}\right)
$$

and one obtains

$$
\begin{aligned}
& R^{(11)}=R^{(d)}+e^{-2 \varphi_{1}(x)} R^{(1)}+e^{-2 \varphi_{2}(x)} R^{(2)}-2 d_{1} \nabla_{\mu} \partial^{\mu} \varphi_{1}(x)+ \\
& +d_{1}\left(d_{1}-1\right) \partial_{\mu} \varphi_{1}(x) \partial^{\mu} \varphi_{1}(x)+-2 d_{2} \nabla_{\mu} \partial^{\mu} \varphi_{2}(x)+ \\
& +d_{2}\left(d_{2}-1\right) \partial_{\mu} \varphi_{2}(x) \partial^{\mu} \varphi_{2}(x)+2 d_{1} d_{2} \partial_{\mu} \varphi_{1}(x) \partial^{\mu} \varphi_{2}(x)
\end{aligned}
$$


where $d_{i}=\operatorname{dim} M^{(i)}$.

Since the covariant derivative is with respect to the whole $g^{(11)}$ metric, two of the terms above are total derivatives and the action is then equivalent to:

$$
\begin{aligned}
& S=\frac{1}{2 \kappa_{11}^{2}} \int \mathrm{d}^{11} x \sqrt{-g^{(11)}}\left(R^{(11)}-V\right) \sim \\
& \sim \frac{1}{2 \kappa_{11}^{2}} \int \mathrm{d}^{11} x \sqrt{-g^{(11)}}\left(R^{(d)}+e^{-2 \varphi_{1}(x)} R^{(1)}+e^{-2 \varphi_{2}(x)} R^{(2)}+\right. \\
& +d_{1}\left(d_{1}-1\right) \partial_{\mu} \varphi_{1}(x) \partial^{\mu} \varphi_{1}(x)+d_{2}\left(d_{2}-1\right) \partial_{\mu} \varphi_{2}(x) \partial^{\mu} \varphi_{2}(x)+ \\
& \left.2 d_{1} d_{2} \partial_{\mu} \varphi_{1}(x) \partial^{\mu} \varphi_{2}(x)-V\right)
\end{aligned}
$$

Step b The eleven-dimensional determinant can be written as

$$
\operatorname{det} g^{(11)}=\operatorname{det} g^{(1)} \operatorname{det} g^{(2)} \operatorname{det} g^{(d)} e^{d_{1} \varphi_{1}+d_{2} \varphi_{2}}
$$

in particular we can integrate over the internal coordinates and get:

$$
\begin{aligned}
S=\frac{V_{1} V_{2}}{2 \kappa_{11}^{2}} \int \mathrm{d}^{d} x \sqrt{-g^{(d)}} e^{d_{1} \varphi_{1}+d_{2} \varphi_{2}}\left(R^{(d)}+e^{-2 \varphi_{1}(x)} R^{(1)}+e^{-2 \varphi_{2}(x)} R^{(2)}+\right. \\
+d_{1}\left(d_{1}-1\right) \partial_{\mu} \varphi_{1}(x) \partial^{\mu} \varphi_{1}(x)+d_{2}\left(d_{2}-1\right) \partial_{\mu} \varphi_{2}(x) \partial^{\mu} \varphi_{2}(x)+ \\
\left.+2 d_{1} d_{2} \partial_{\mu} \varphi_{1}(x) \partial^{\mu} \varphi_{2}(x)-V\right)
\end{aligned}
$$

We will introduce

$$
\Psi=d_{1} \varphi_{1}+d_{2} \varphi_{2}
$$

for future purposes.

Step c The action above is not in the usual Hilbert-Einstein form because of the $e^{d_{1} \varphi_{1}+d_{2} \varphi_{2}}$ factor. For this reason we perform a Weyl rescaling

$$
\bar{g}_{\mu v}=e^{2 \sigma(x)} g_{\mu \nu}
$$

under which the curvature becomes:

$$
\bar{R}=e^{-2 \sigma(x)} R-2(d-1) \bar{\nabla}_{\mu} \partial^{\mu} \sigma(x)+(d-1)(d-2) \partial_{\mu} \sigma(x) \partial^{\mu} \sigma(x)
$$

where $\bar{\nabla}$ is the covariant derivative with respect to $\bar{g}$ and $\mu$ is raised with $\bar{g}$.

In $d$ dimensions a term $\sqrt{\operatorname{det} g} e^{\Psi} R$ is brought to the standard form by the Weyl rescaling

$$
\bar{g}_{\mu \nu}=\exp \left[\frac{2}{d-2} \Psi\right] g_{\mu \nu}
$$

and

$$
\sqrt{g} e^{\Psi} R=\sqrt{\bar{g}}\left(\bar{R}+2 \frac{d-1}{d-2} \bar{\nabla} \partial^{\mu} \Psi-\frac{d-1}{d-2} \partial_{\mu} \Psi \partial^{\mu} \Psi\right) .
$$


Discarding the total derivatives, the action now reads

$$
\begin{aligned}
& S=\frac{V_{1} V_{2}}{2 \kappa_{11}^{2}} \int \mathrm{d}^{d} x \sqrt{-\bar{g}^{(d)}}\left[\bar{R}^{(d)}-\frac{d-1}{d-2} \partial_{\mu} \Psi \partial^{\mu} \Psi+\right. \\
& +d_{1}\left(d_{1}-1\right) \partial_{\mu} \varphi_{1}(x) \partial^{\mu} \varphi_{1}(x)+d_{2}\left(d_{2}-1\right) \partial_{\mu} \varphi_{2}(x) \partial^{\mu} \varphi_{2}(x)+ \\
& \left.+2 d_{1} d_{2} \partial_{\mu} \varphi_{1}(x) \partial^{\mu} \varphi_{2}(x)+e^{-2 \Psi_{1} /(d-2)}\left(e^{-2 \varphi_{1}(x)} R^{(1)}+e^{-2 \varphi_{2}(x)} R^{(2)}-V\left(\varphi_{1}, \varphi_{2}\right)\right)\right]
\end{aligned}
$$

where indices are raised with $\bar{g}^{(d)}$.

Step d Now let us collect all the $\varphi$ terms in the action:

$$
\begin{aligned}
S= & \frac{V_{1} V_{2}}{2 \kappa_{11}^{2}} \int \mathrm{d}^{d} x \sqrt{-\bar{g}^{(d)}}\left[\bar{R}^{(d)}-d_{1}\left(\frac{d_{1}}{d-2}+1\right) \partial_{\mu} \varphi_{1} \partial^{\mu} \varphi_{1}+\right. \\
& \left.-d_{2}\left(\frac{d_{2}}{d-2}+1\right) \partial_{\mu} \varphi_{2} \partial^{\mu} \varphi_{2}-\frac{2 d_{1} d_{2}}{d-2} \partial_{\mu} \varphi_{1} \partial^{\mu} \varphi_{2}-\bar{V}\left(\varphi_{1}, \varphi_{2}\right)\right]
\end{aligned}
$$

where

$$
\bar{V}\left(\varphi_{1}, \varphi_{2}\right)=e^{-2\left(d_{1} \varphi_{1}+d_{2} \varphi_{2}\right) /(d-2)}\left(-e^{-2 \varphi_{1}(x)} R^{(1)}-e^{-2 \varphi_{2}(x)} R^{(2)}+V\left(\varphi_{1}, \varphi_{2}\right)\right) .
$$

To bring the kinetic terms to the standard form we introduce:

$$
\left\{\begin{array}{l}
\Phi_{1}=\sqrt{\frac{2(D-2)}{\left(d_{1}+d_{2}\right)(d-2)}}\left(d_{1} \varphi_{1}+d_{2} \varphi_{2}\right) \\
\Phi_{2}=\sqrt{\frac{2 d_{1} d_{2}}{d_{1}+d_{2}}}\left(\varphi_{1}-\varphi_{2}\right)
\end{array}\right.
$$

or, the other way round:

$$
\left\{\begin{array}{l}
\varphi_{1}=\frac{1}{\sqrt{2\left(d_{1}+d_{2}\right)}}\left(\sqrt{\frac{d-2}{D-2}} \Phi_{1}+\sqrt{\frac{d_{2}}{d_{1}}} \Phi_{2}\right) \\
\varphi_{1}=\frac{1}{\sqrt{2\left(d_{1}+d_{2}\right)}}\left(\sqrt{\frac{d-2}{D-2}} \Phi_{1}-\sqrt{\frac{d_{1}}{d_{2}}} \Phi_{2}\right)
\end{array}\right.
$$

where $D=d+d_{1}+d_{2}=11$, and we finally obtain

$$
S=\frac{V_{1} V_{2}}{2 \kappa_{11}^{2}} \int \mathrm{d}^{d} x \sqrt{-g^{(d)}}\left[R^{(d)}-\frac{1}{2} \partial_{\mu} \Phi_{1} \partial^{\mu} \Phi_{1}-\frac{1}{2} \partial_{\mu} \Phi_{2} \partial^{\mu} \Phi_{2}-\bar{V}\left(\Phi_{1}, \Phi_{2}\right)\right]
$$

Step e The type of backgrounds we obtain after compactification are AdS. Therefore, negative- $m^{2}$ modes are not tachyonic if they don't cross the BreitenlohnerFreedman bound.

Using the results of the previous section this means:

$$
M^{2}=-\frac{d-1}{4(d-2)}\langle V\rangle+m^{2} \geq 0
$$


that must be respected by each eigenvalue $m^{2}$ of the Hessian matrix

$$
m_{i j}^{2}=\left.\frac{\partial^{2} V}{\partial \Phi_{i} \partial \Phi_{j}}\right|_{V=V_{0}}
$$

These general considerations apply to any choice of background gauge fields. Then one should write the potential for each case at hand in terms of $\Phi_{1}$ and $\Phi_{2}$ (and possibly the dilaton in type II) and check the mass matrix against the BF bound.

$\mathrm{AdS}_{4} \times H^{3} \times S^{4}$

As a first example let us consider $\mathrm{AdS}_{4} \times H^{3} \times S^{4}$. The potential $V$ in Eq. (7.45) is due to the presence of a four-form field on the $S^{4}$ part.

$$
V\left(\varphi_{1}, \varphi_{2}\right)=\frac{Q^{2}}{2} e^{-8 \varphi_{2}}
$$

the dimensions are $d=4, d_{1}=3$ and $d_{2}=4$ and the curvatures read $R^{(1)}=$ $-3 / 2$ and $R^{(2)}=2$, so the expression in Eq. (7.61) becomes:

$$
\bar{V}\left(\varphi_{1}, \varphi_{2}\right)=e^{-3 \varphi_{1}-4 \varphi_{2}}\left(\frac{3}{2} e^{-2 \varphi_{1}}-2 e^{\varphi_{2}}+\frac{Q^{2}}{2} e^{-8 \varphi_{2}}\right) .
$$

To get the canonical scalar fields $\Phi_{i}$ and $\Phi_{2}$ we can use Eq. (7.63):

$$
\left\{\begin{array}{l}
\varphi_{1}=\frac{1}{3 \sqrt{7}}\left(\Phi_{1}+\sqrt{6} \Phi_{2}\right) \\
\varphi_{2}=\frac{1}{12 \sqrt{7}}\left(4 \Phi_{1}-3 \sqrt{6} \Phi_{2}\right)
\end{array}\right.
$$

and this leads to the effective potential

$$
V\left(\Phi_{1}, \Phi_{2}\right)=\frac{1}{2} e^{-5 \Phi_{1} / \sqrt{7}-2 \Phi_{2} \sqrt{2 / 21}}\left(3 e^{2 \Phi_{1} / \sqrt{7}}-4 e^{2 \Phi_{1} / \sqrt{7}+\Phi_{2} \sqrt{7 / 6}}+Q^{2} e^{8 \Phi_{2} \sqrt{2 / 21}}\right)
$$

which has a minimum for

$$
\Phi_{1}=\sqrt{7} \log \frac{2^{8 / 7} Q}{\sqrt{3}} \quad \Phi_{2}=\sqrt{\frac{6}{7}} \log 2
$$

and in correspondence of this point

$$
\langle V\rangle=-\frac{3 \sqrt{3}}{16 Q^{3}} .
$$

The Hessian matrix on the minimum is:

$$
\frac{\partial^{2} V}{\partial \Phi_{i} \partial \Phi_{j}}=\frac{3}{28 Q^{3}}\left(\begin{array}{cc}
\frac{15 \sqrt{3}}{4} & -\frac{9}{\sqrt{2}} \\
-\frac{9}{\sqrt{2}} & \frac{17 \sqrt{3}}{2}
\end{array}\right)
$$

Both eigenvalues are positive

$$
\left.m^{2}\right|_{1}=\left.\frac{3 \sqrt{3}}{16 Q^{3}} \quad m^{2}\right|_{2}=\frac{9 \sqrt{3}}{8 Q^{3}}
$$

and the solution is stable. 


\begin{tabular}{|c|c|c|c|c|c|}
\hline$d_{1}$ & $\left\langle\Phi_{1}\right\rangle$ & $\left\langle\Phi_{2}\right\rangle$ & $\langle V\rangle$ & $\frac{\left.m^{2}\right|_{1}}{\langle V\rangle}$ & $\frac{\left.m^{2}\right|_{2}}{\langle V\rangle}$ \\
\hline \hline 2 & $\log \frac{4 Q^{2}}{3}$ & $\sqrt{\frac{2}{3}} \log 2$ & $-\frac{9}{8 \times 2^{2 / 3} Q^{2}}$ & $2 / 3$ & 4 \\
\hline 3 & $\frac{\sqrt{7}}{2} \log \frac{4^{8 / 7} Q^{2}}{3}$ & $\sqrt{\frac{6}{7}} \log 2$ & $-\frac{3 \sqrt{3}}{16 Q^{3}}$ & 1 & 6 \\
\hline 4 & $2 \log \frac{4 \sqrt{2} Q^{2}}{3}$ & $\log 2$ & $-\frac{27}{512 Q^{6}}$ & 2 & 12 \\
\hline
\end{tabular}

Table 7.1: Minima and masses for AdS $\times H$ backgrounds

$\mathrm{AdS}_{7-d_{1}} \times H^{d_{1}} \times S^{4}$

All the backgrounds of the form $\mathrm{AdS}_{7-d_{1}} \times H^{d_{1}} \times S^{4}$ can be treated similarly. The potential in Eq. (7.61) becomes:

$$
\bar{V}\left(\varphi_{1}, \varphi_{2}\right)=e^{-2\left(d_{1} \varphi_{1}-4 \varphi_{2}\right) /\left(5-d_{1}\right)}\left(-\frac{d_{1}}{2} e^{-2 \varphi_{1}}-2 e^{\varphi_{2}}+\frac{Q^{2}}{2} e^{-8 \varphi_{2}}\right)
$$

and one finds that in each case there is a minimum (stable) solution. The actual values are in Tab. 7.1. In any case, not surprisingly one can see that for any $d_{1}$ the warping factors $\varphi_{1}$ and $\varphi_{2}$ always take the values

$$
\varphi_{1}=\frac{1}{6}\left(\log \frac{16 Q^{2}}{3}\right) \quad \varphi_{2}=\frac{1}{6}\left(\log \frac{3 Q^{2}}{2}\right)
$$

which agrees with $\Lambda_{2}$ and $\Lambda_{3}$ being

$$
\Lambda_{2}=-\left(\frac{3}{2}\right)^{1 / 3} \frac{1}{2 Q^{2 / 3}} \quad \Lambda_{3}=\left(\frac{3}{2}\right)^{1 / 3} \frac{1}{Q^{2 / 3}}
$$

as we have already seen by directly solving the equations of motion in eleven dimensions.

$\mathrm{AdS}_{4} \times S^{3} \times S^{4}$

Consider now $\mathrm{AdS}_{4} \times S^{3} \times S^{4}$. The potential $V$ in Eq. (7.45) is due to the presence of a seven-form field on the $S^{3} \times S^{4}$ factor.

$$
V\left(\varphi_{1}, \varphi_{2}\right)=\frac{Q^{2}}{2} e^{-6 \varphi_{1}-8 \varphi_{2}} .
$$

The dimensions are $d=4, d_{1}=3$ and $d_{2}=4$ and the curvatures read $R^{(1)}=$ $3 / 2$ and $R^{(2)}=2$, so the expression in Eq. (7.61) becomes:

$$
\bar{V}\left(\varphi_{1}, \varphi_{2}\right)=e^{-3 \varphi_{1}-4 \varphi_{2}}\left(-\frac{3}{2} e^{-2 \varphi_{1}}-2 e^{\varphi_{2}}+\frac{Q^{2}}{2} e^{-6 \varphi_{1}-8 \varphi_{2}}\right) .
$$

To get the canonical scalar fields $\Phi_{i}$ and $\Phi_{2}$ we can use Eq. (7.63):

$$
\left\{\begin{array}{l}
\varphi_{1}=\frac{1}{3 \sqrt{7}}\left(\Phi_{1}+\sqrt{6} \Phi_{2}\right) \\
\varphi_{2}=\frac{1}{12 \sqrt{7}}\left(4 \Phi_{1}-3 \sqrt{6} \Phi_{2}\right)
\end{array}\right.
$$


and this leads to the effective potential

$$
V\left(\Phi_{1}, \Phi_{2}\right)=\frac{1}{2} e^{-\sqrt{7} \Phi_{1}}\left(-3 e^{4 \Phi_{1} / \sqrt{7}-2 \Phi_{2} \sqrt{2 / 21}}-4 e^{4 \Phi_{1} / \sqrt{7}+\Phi_{2} \sqrt{3 / 14}}+Q^{2}\right)
$$

In this case there is an extremum for

$$
\Phi_{1}=\frac{\sqrt{7}}{4} \log \frac{Q^{2}}{3} \quad \Phi_{2}=0
$$

and in correspondence of this point:

$$
\langle V\rangle=-2 \frac{3^{3 / 4}}{Q^{3 / 2}} .
$$

To verify the stability we write the Hessian matrix:

$$
\frac{\partial^{2} V}{\partial \Phi_{i} \partial \Phi_{j}}=2 \frac{3^{3 / 4}}{Q^{3 / 2}}\left(\begin{array}{cc}
3 & 0 \\
0 & -\frac{1}{2}
\end{array}\right)
$$

which possesses a negative eigenvalue

$$
\left.m^{2}\right|_{1}=\left.3|\langle V\rangle| \quad m^{2}\right|_{2}=-\frac{1}{2}|\langle V\rangle| .
$$

This must be confronted with the BF bound we found in Eq. (7.65):

$$
M^{2}=\frac{3}{8}|\langle V\rangle|+m^{2}=\left(\frac{3}{8}-\frac{1}{2}\right)|\langle V\rangle|=-\frac{1}{8}|\langle V\rangle|<0 .
$$

The mode, corresponding to the ratio of the two radii $\Phi_{2}$, is actually unstable. This is not so surprising since in this case the flux is proportional to the total volume of the compact directions and the system is intrinsically unstable with respect to a perturbation that woukd keep this volume constant while changing the ratio between the two radii.

$\mathrm{AdS}_{4} \times S^{d_{1}} \times S^{7-d_{1}}$

The field choice we made above proves to be very useful when dealing with general $\mathrm{AdS}_{4} \times S^{d_{1}} \times S^{7-d_{1}}$ backgrounds. In fact the potential reads:

$$
\begin{aligned}
V\left(\Phi_{1}, \Phi_{2}\right)=\frac{1}{2} e^{-\sqrt{7} \Phi_{1}}( & -d_{1} e^{4 \Phi_{1} / \sqrt{7}-\Phi_{2} \sqrt{\left(-2 d_{1}+14\right) /\left(7 d_{1}\right)}}+ \\
& \left.-\left(7-d_{1}\right) e^{4 \Phi_{1} / \sqrt{7}+\Phi_{2} \sqrt{2 d_{1} /\left(7\left(d_{1}-7\right)\right)}}+Q^{2}\right)
\end{aligned}
$$

where $d_{1}$ appears only as a coefficient for $\Phi_{2}$. Therefore the solution is independent of the dimension $d_{1}$ and the stationary point is again

$$
\Phi_{1}=\frac{\sqrt{7}}{4} \log \frac{Q^{2}}{3} \quad \Phi_{2}=0
$$


with

$$
\langle V\rangle=-2 \frac{3^{3 / 4}}{Q^{3 / 2}}
$$

This solution is again unstable, since the square masses are again

$$
\left.m^{2}\right|_{1}=\left.3|\langle V\rangle| \quad m^{2}\right|_{2}=-\frac{1}{2}|\langle V\rangle|
$$

and the BF bound is always for $-3 / 8|\langle V\rangle|$ since it only depends on the noncompact dimensions that are $d=4$.

\subsection{Type IIB backgrounds}

Some of the solutions we found thus far can be naturally reduced to type II. This is the case when they contain factors of $\mathrm{AdS}_{3}$ or odd-dimensional spheres for they can be respectively written as space-like fibrations over $\mathrm{AdS}_{2}$ or complex projective planes and as such, when compactified on the fiber don't give rise to dilaton fields.

On the other hand one might also directly look for type II solutions with the same type of geometry factorized in constant curvature spaces. In this section we will in particular concentrate on type IIB solutions with structure $V_{4} \times M_{3} \times \tilde{M}_{3}$. Before starting one can try to make some educated guesses about the expected kind of solutions. It would then appear rather natural to expect perturbative $\mathrm{AdS}_{4} \times S^{3} \times S^{3}$ solutions that might prove to be unstable (in the same spirit as in $\left[\mathrm{DFG}^{+} 02\right]$ ) and one might further imagine that adding non-perturbative objects with negative tension - orientifold planes - the no-go theorem for de Sitter [MN01] can be contoured, thus allowing for internal hyperbolic manifolds and for de Sitter solutions (as it was suggested in [SS06]). In fact we will prove that both guesses are ultimately wrong by carefully studying the effective potential in four dimensions obtained by taking into account dilaton, RR zero-form and the two breathing modes for the internal manifolds. More precisely we will show that:

- no truly perturbative solution exists, i.e. the presence of D-branes is necessary;

- in presence of orientifolds the only possible compactification happens on a $T^{6}$

- no solution is allowed with hyperbolic or de Sitter components;

- the only allowed solution is $\mathrm{AdS}_{4} \times S^{3} \times S^{3}$ and this solution is perturbatively stable, thanks to the BF bound, although it can't be completely trusted since it belongs to an intermediate-coupling regime. 
Let us consider a type IIB background of the form $V_{4} \times M_{3} \times \tilde{M}_{3}$. The action in Einstein frame reads:

$$
\begin{aligned}
& S=S_{0}+S_{\text {loc }}=\frac{1}{2 \kappa_{10}^{2}} \int \mathrm{d}^{10} x \sqrt{-g^{(10)}}\left[R^{(10)}-\frac{\partial_{\mu} \tau \partial^{\mu} \bar{\tau}}{2(\operatorname{Im} \tau)^{2}}-\frac{1}{2} M_{i j} F_{3}^{i} F_{3}^{j}+\right. \\
& \left.-\frac{1}{2} C_{4} \wedge F_{3}^{1} \wedge F_{3}^{2}\right]+S_{\mathrm{loc}}
\end{aligned}
$$

where we didn't include the 1-form and the 5-form which are not compatible with the symmetries of the metric ansatz. As usual $\tau=C_{0}+\imath e^{-\Phi}$ is the dilaton-axion field, $M_{i j}$ is

$$
M_{i j}=\frac{1}{\operatorname{Im} \tau}\left(\begin{array}{cc}
|\tau|^{2} & -\operatorname{Re} \tau \\
-\operatorname{Re} \tau & 1
\end{array}\right)
$$

and $S_{\text {loc }}$ is the contribution due to $D_{3}$-branes and $O_{3}$-planes:

$$
S_{\mathrm{loc}}=N T_{3} \int \mathrm{d}^{4} x \sqrt{-g^{(4)}} .
$$

As before we look for a solution of the kind

$$
\mathrm{d} s_{(10)}^{2}=\mathrm{d} s_{(4)}^{2}+e^{2 \varphi(x)} \mathrm{d} s_{3}^{2}+e^{2 \tilde{\varphi}(x)} \mathrm{d} s_{(3)}^{2}
$$

where for the moment being the two internal manifolds can have positive or negative curvature. With respect to the M-theory situation, in this case we will have to pay attention to the extra $\tau$ complex scalar field and to the extra-term in the action $S_{\text {loc }}$.

Let's start with the latter. This already has the form of a four-dimensional integral, so to evaluate the corresponding contribution to the potential we only need to take into account the Weyl rescaling in Eq. (7.57) and the presence of the internal volumes and Newton constant:

$$
S_{\text {loc }}=N T_{3} \int \mathrm{d}^{4} x \sqrt{-g^{(4)}}=\frac{V_{3} \tilde{V}_{3}}{2 \kappa_{10}^{2}} \frac{2 N T_{3} \kappa_{10}^{2}}{V_{3} \tilde{V}_{3}} \int \mathrm{d}^{4} x e^{-2 \Psi_{1}} \sqrt{\bar{g}^{(4)}}
$$

and then

$$
V_{\mathrm{loc}}=\frac{2 N T_{3} \kappa_{10}^{2}}{V_{3} \tilde{V}_{3}} e^{-6 \varphi-6 \tilde{\varphi}}
$$

For reasons that will appear more clear in the following let us rescale the $\tau$ field as:

$$
z=\frac{\tau}{k}
$$

$k$ being a real constant. This does not affect the kinetic term:

$$
\frac{\partial_{\mu} \tau \partial^{\mu} \bar{\tau}}{2(\operatorname{Im} \tau)^{2}}=\frac{\partial_{\mu} z \partial^{\mu} \bar{z}}{2(\operatorname{Im} z)^{2}}
$$


but makes it canonical when studying the small perturbations around the equilibrium solutions.

The most general three-field configuration compatible with the symmetries is

$$
F_{3}^{i}=4 \pi^{2} \alpha^{\prime}\left(n_{i} \omega_{3}+\tilde{n}_{i} \tilde{\omega}_{3}\right)
$$

where $n_{i}$ and $\tilde{n}_{i}$ are integers and $\omega_{3}$ and $\tilde{\omega}_{3}$ are the normalized volume forms. The subscript 1 stands for RR field, 2 for NS. The corresponding potential reads:

$$
V_{F}=\frac{\left(4 \pi^{2}\right)^{2} \alpha^{\prime 2}}{2 k \operatorname{Im} z V_{3}^{2}}\left|n_{1} k z-n_{2}\right|^{2} e^{-6 \varphi}+\frac{\left(4 \pi^{2}\right)^{2} \alpha^{\prime 2}}{2 k \operatorname{Im} z \tilde{V}_{3}^{2}}\left|\tilde{n}_{1} k z-\tilde{n}_{2}\right|^{2} e^{-6 \tilde{\varphi}}
$$

in order to clean the notation let us write the volumes as

$$
V_{3}=2 x \pi^{2} \quad \tilde{V}_{3}=2 \tilde{x} \pi^{2} .
$$

Adding the contribution from the curvatures of the internal manifolds:

$$
R=\frac{3}{2} \epsilon \quad \tilde{R}=\frac{3}{2} \tilde{\epsilon}
$$

where $\epsilon=1$ for spheres and $\epsilon=-1$ for hyperbolic spaces, we can write the potential as

$$
\begin{aligned}
V(\varphi, \tilde{\varphi}, z)= & e^{-3 \varphi-3 \tilde{\varphi}}\left(-\frac{3 \epsilon}{2} e^{-2 \varphi}-\frac{3 \tilde{\epsilon}}{2} e^{-2 \tilde{\varphi}}+\frac{2 \alpha^{\prime 2}}{x^{2} k \operatorname{Im} z}\left|n_{1} k z-n_{2}\right|^{2} e^{-6 \varphi}+\right. \\
& \left.+\frac{2 \alpha^{\prime 2}}{x^{2} k \operatorname{Im} z}\left|\tilde{n}_{1} k z-\tilde{n}_{2}\right|^{2} e^{-6 \tilde{\varphi}}\right)+\frac{2 N T_{3} \kappa_{10}^{2}}{x \tilde{x}\left(2 \pi^{2}\right)^{2}} e^{-6 \varphi-6 \tilde{\varphi}}
\end{aligned}
$$

As a first step we normalize the scalars $\varphi$ and $\tilde{\varphi}$ :

$$
\varphi=\frac{\Phi_{1}+2 \Phi_{2}}{4 \sqrt{3}} \quad \tilde{\varphi}=\frac{\Phi_{1}-2 \Phi_{2}}{4 \sqrt{3}}
$$

so to rewrite the potential as

$$
\begin{aligned}
& V\left(\Phi_{1}, \Phi_{2}, z\right)=e^{-\Phi_{1} \sqrt{3} / 2}\left(-\frac{3}{2} \epsilon e^{-\left(\Phi_{1}+2 \Phi_{2}\right) /(2 \sqrt{3})}-\frac{3}{2} \epsilon e^{-\left(\Phi_{1}-2 \Phi_{2}\right) /(2 \sqrt{3})}+\right. \\
& +\frac{2 \alpha^{\prime 2}}{x^{2} k \operatorname{Im} z}\left|n_{1} k z-n_{2}\right|^{2} e^{-\left(\Phi_{1}+2 \Phi_{2}\right) \sqrt{3} / 2}+ \\
& \left.\quad \frac{2 \alpha^{\prime 2}}{x^{2} k \operatorname{Im} z}\left|\tilde{n}_{1} k z-\tilde{n}_{2}\right|^{2} e^{-\left(\Phi_{1}-2 \Phi_{2}\right) \sqrt{3} / 2}\right)+\frac{2 N T_{3} \kappa_{10}^{2}}{x \tilde{x}\left(2 \pi^{2}\right)^{2}} e^{-\Phi_{1} \sqrt{3}} .
\end{aligned}
$$

We have to take into account the anomaly cancellation condition

$$
N=n_{1} \tilde{n}_{2}-n_{2} \tilde{n}_{1}
$$

and express all the constants in terms of $\alpha^{\prime}$ :

$$
T_{3}=\left|\mu_{3}\right|=\frac{1}{(2 \pi)^{2} \alpha^{\prime 2}} \quad 2 \kappa_{10}^{2}=(2 \pi)^{7} \alpha^{4}
$$


so that $2 \kappa_{10}^{2} T_{3}=(2 \pi)^{4} \alpha^{\prime 2}$.

Up to this moment the sign of $N$ is arbitrary, so one might think that different kinds of solution are possible. Actually this is not the case. If we differentiate with respect to $\operatorname{Im} z$ and $\operatorname{Re} z$ we obtain the values corresponding to the stationary point

$$
\operatorname{Im} z=\frac{e^{\Phi_{2} \sqrt{3}}|N| x \tilde{x}}{k\left(e^{2 \Phi_{2} \sqrt{3}} \tilde{n}_{1}^{2} x^{2}+n_{1}^{2} \tilde{x}^{2}\right)} \quad \operatorname{Re} z=\frac{e^{2 \Phi_{2} \sqrt{3}} \tilde{n}_{1} \tilde{n}_{2} x^{2}+n_{1} n_{2} \tilde{x}^{2}}{k\left(e^{2 \Phi_{2} \sqrt{3}} \tilde{n}_{1}^{2} x^{2}+n_{1}^{2} \tilde{x}^{2}\right)}
$$

Putting these back into the potential one gets:

$V\left(\Phi_{1}, \Phi_{2}\right)=\frac{e^{\left(3 \Phi_{1}+\Phi_{2}\right) / \sqrt{3}}}{2 x \tilde{x}}\left(8 e^{\Phi_{2} / \sqrt{3}}(N+|N|)-3 x \tilde{x} e^{\Phi_{1} / \sqrt{3}}\left(\epsilon+\tilde{\epsilon} e^{\left(\Phi_{1}+2 \Phi_{2}\right) / \sqrt{3}}\right)\right)$

and for $N \gtrless 0$ this gives respectively:

$$
\begin{aligned}
& V^{+}\left(\Phi_{1}, \Phi_{2}\right)=\frac{e^{\left(3 \Phi_{1}+\Phi_{2}\right) / \sqrt{3}}}{2 x \tilde{x}}\left(16 e^{\Phi_{2} / \sqrt{3}} N-3 x \tilde{x} e^{\Phi_{1} / \sqrt{3}}\left(\epsilon+\tilde{\epsilon} e^{\left(\Phi_{1}+2 \Phi_{2}\right) / \sqrt{3}}\right)\right) \\
& V^{-}\left(\Phi_{1}, \Phi_{2}\right)=-\frac{3}{2} e^{-\left(2 \Phi_{1}+\Phi_{2}\right) / \sqrt{3}}\left(\epsilon+\tilde{\epsilon} e^{2 \Phi_{2} / \sqrt{3}}\right) .
\end{aligned}
$$

The second potential only admits a solution if $\epsilon=\tilde{\epsilon}=0$, but this corresponds to a flat internal space (Polchinski).

Choosing the first $N>0$ case one finds a stationary point for

$$
\left\langle\Phi_{1}\right\rangle=\sqrt{3} \log \frac{4 N \alpha^{\prime 2}}{x \tilde{x} \tilde{\epsilon}} \sqrt{\frac{\tilde{\epsilon}}{\epsilon}} \quad\left\langle\Phi_{2}\right\rangle=\frac{\sqrt{3}}{2} \log \frac{\epsilon}{\tilde{\epsilon}}
$$

which imposes $\epsilon=\tilde{\epsilon}=1$, ie the only solution has an internal $S^{3} \times S^{3}$ space, as advertized above.

To summarize, the stationary point corresponds to:

$$
\begin{array}{rr}
\langle\operatorname{Im} z\rangle=\frac{N x \tilde{x}}{k\left(\tilde{n}_{1}^{2} x^{2}+n_{1}^{2} \tilde{x}^{2}\right)} & \langle\operatorname{Re} z\rangle=\frac{\tilde{n}_{1} \tilde{n}_{2} x^{2}+n_{1} n_{2} \tilde{x}^{2}}{k\left(\tilde{n}_{1}^{2} x^{2}+n_{1}^{2} \tilde{x}^{2}\right)} \\
\left\langle\Phi_{1}\right\rangle=\sqrt{3} \log \frac{4 N \alpha^{\prime 2}}{x \tilde{x}} & \left\langle\Phi_{2}\right\rangle=0
\end{array}
$$

now we can choose

$$
k=\frac{N x \tilde{x}}{\tilde{n}_{1}^{2} x^{2}+n_{1}^{2} \tilde{x}^{2}}
$$

and putting $x=\tilde{x}=1$ (as we must in the case of spheres) we have

$$
\langle\operatorname{Im} z\rangle=1 \quad\langle\operatorname{Re} z\rangle=\frac{\tilde{n}_{1} \tilde{n}_{2}+n_{1} n_{2}}{n_{1} \tilde{n}_{2}-n_{2} \tilde{n}_{1}} \quad\left\langle\Phi_{1}\right\rangle=\sqrt{3} \log \left(4 N \alpha^{\prime 2}\right) \quad\left\langle\Phi_{2}\right\rangle=0
$$


and the corresponding potential is

$$
\langle V\rangle=-\frac{1}{16 N^{2} \alpha^{\prime 4}}
$$

To check the stability we write the Hessian matrix and compute its eigenvalues. They turn out to be

$$
\begin{array}{rr}
\left.m^{2}\right|_{1}=|\langle V\rangle| & \left.m^{2}\right|_{2}=2|\langle V\rangle| \\
\left.m^{2}\right|_{3}=\frac{\sqrt{13}+3}{2}|\langle V\rangle| & \left.m^{2}\right|_{4}=-\frac{\sqrt{13}-3}{2}|\langle V\rangle|
\end{array}
$$

and the only negative one $\left.m^{2}\right|_{4}$ doesn't cause any instability since it doesn't cross the BF bound:

$$
\left.m^{2}\right|_{4}+\frac{3}{8}|\langle V\rangle|=\frac{15-4 \sqrt{13}}{8}|\langle V\rangle|>0
$$

This result about the stability of the product of two three-spheres can at first sight be puzzling since, after analyzing the results of Sec. 7.2, we've grown to expect such configurations to be unstable under the mode in which one of the two spheres shrinks and the other grows, keeping constant the overall volume. Here it is not the case and this can be easily understood in terms of gauge fields. In the M-theory configurations, in fact, only one field was turned on and it spanned over the whole internal manifold, so that it effected only the total volume. Here, on the other hand, RR and NS-NS fields are turned on separately on the two submanifolds, thus contributing to the stabilization of each of the radii. 


\section{CHAPTER 8}

\section{Conclusions and further perspectives}

Beauty is truth, truth beauty, - that is all Ye know on earth, and all ye need to know.

Ode on a Grecian Urn JOHN KEATS

The search for exact string solutions is a fascinating subject by itself. It is based, as most of the wonders of string theory, on the interplay between the two-dimensional conformal field theory description on the world-sheet and the ten-dimensional low-energy interpretation in terms of spacetime fields.

In this thesis we have dealt with a new class of string backgrounds living in the moduli space of WZW models. They enjoy at the same time nice supergravity properties, all geometrical quantities being naturally expressed in terms of algebraic invariants, and a clear CFT characterization, inherited from the beautiful theory of group manifolds.

Apart from their intrinsic elegance, those new backgrounds also find interesting physical applications as compactification manifolds, laboratories for the analysis of string propagation in classically pathological backgrounds with closed time-like curves, in black hole configurations with non-trivial topology. Laboratories in which we can keep higher order effects under control and write down a modular-invariant partition function, or at least the spectra of primary operators.

Starting from this solid CFT ground we are then allowed to peep into the off-shell physics using RG techniques, both from a two-dimensional and fieldtheoretical perspective. We can thus observe the relaxation of out-of-equilibrium vacua described by charge transmutation, i.e. two gauge fields eventually collapsing into a single one, while the total charge is conserved. A new change of viewpoint then allows us to recast the problem in terms of a cosmological time-dependent solution. The RG dynamics becomes an approximate description - valid in a certain region of the moduli space depending on the central charge deficit - for the behaviour of a Big-bang-like isotropic FRW universe.

We have emphasized many times the importance of having a CFT description and exact models, but this is not possible in general. For example we 
completely lack such a kind of interpretation for type II string or M-theory, but - and maybe for this very reason - it is important to look for new insights in these frameworks. In this spirit we have studied compactifications involving maximally symmetric spaces which in general do not preserve supersymmetry, looking in particular for hyperbolic solutions, in the not-too-concealed hope of reaching de Sitter-like spacetimes.

So, what remains to do? By its very nature this is a work in progress. The path to further developments is full of technical and conceptual obstacles but one can easily name some of the natural possible directions. First of all it would be interesting to have a non-Abelian counterpart for our asymmetric gauging. This would be allowed by the heterotic string framework and it does indeed work at the supergravity level; on the other hand it is not clear how it could be implemented in a CFT framework - the evidence at hand pointing towards a discrete structure for the deformations. Then, one would like to reach a better understanding of non-rational CFTs and, in particular, of the $S L(2, \mathbb{R})$ WZW model; this would allow us to write the partition function for $\mathrm{AdS}_{2}$ spacetime, Bertotti-Robinson black hole and (charged) black string. This, in turn, might prove useful for obtaining a non-trivial microscopic description for the thermodynamics of such singular objects. Another more phenomenological direction would be to study the low energy field theory consequence of a compactification on geometric cosets, again using the complete knowledge which we have of the spectrum and partition function in this case. As soon as we move away from the familiar CFT framework things become more difficult and much more interesting. Even if a theory for the two-dimensional RG flow is established, there are very few cases in which one can really work out non-trivial examples such as the flow one expects to link WZW models at different levels. The spacetime description might then prove useful for getting new hints at the world-sheet physics. 


\section{Appendix A}

\section{Table of conventions}

Christoffel symbol

covariant derivative

dual Coxeter number

exterior derivative

Maurer-Cartan one-form

n-form

structure constants

trace

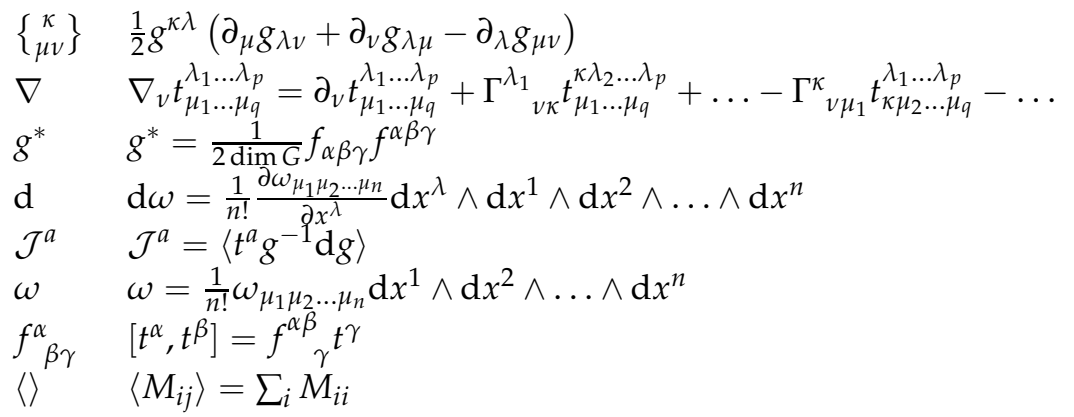





\section{APPENDix B}

\section{Explict parametrizations for some Lie groups}

In this appendix we collect the explicit parametrizations used for the SU(2), $S L(2, R), S U(3)$ and $U S p(4)$ groups.

\section{B.1 The three-sphere}

The commutation relations for the generators of $S U(2)$ are

$$
\left[J^{1}, J^{2}\right]=\imath J^{3} \quad\left[J^{2}, J^{3}\right]=\imath J^{1} \quad\left[J^{3}, J^{1}\right]=\imath J^{2} .
$$

A two-dimensional realization is obtained by using the standard Pauli matrices ${ }^{1} \sigma^{a}$ : $J^{a}=\sigma^{a} / 2$.

The Euler-angle parameterization for $S U(2)$ is defined as:

$$
g=e^{l \frac{\gamma}{2} \sigma^{3}} e^{l \frac{\beta}{2} \sigma^{1}} e^{l \frac{\alpha}{2} \sigma^{3}} .
$$

The $S U(2)$ group manifold is a unit-radius three-sphere. A three-sphere can be embedded in flat Euclidean four-dimensional space with coordinates $\left(x^{1}, x^{2}, x^{3}, x^{4}\right)$, as $\left(x^{1}\right)^{2}+\left(x^{2}\right)^{2}+\left(x^{3}\right)^{2}+\left(x^{4}\right)^{2}=L^{2}$. The corresponding SU(2) element $g$ is the following:

$$
g=L^{-1}\left(\begin{array}{cc}
x^{4}+\imath x^{2} & x^{3}+\imath x^{1} \\
-x^{3}+\imath x^{1} & x^{4}-\imath x^{2}
\end{array}\right)
$$

In general, the invariant metric of a group manifold can be expressed in terms of the left-invariant Cartan-Maurer one-forms. In the $S U(2)$ case under consideration (unit-radius $S^{3}$ ),

$$
\mathcal{J}^{1}=\frac{1}{2} \operatorname{tr}\left(\sigma^{1} g^{-1} \mathrm{~d} g\right), \quad \mathcal{J}^{2}=\frac{1}{2} \operatorname{tr}\left(\sigma^{2} g^{-1} \mathrm{~d} g\right), \quad \mathcal{J}^{3}=\frac{1}{2} \operatorname{tr}\left(\sigma^{3} g^{-1} \mathrm{~d} g\right)
$$

and

$$
\mathrm{d} s^{2}=\sum_{i=1}^{3} \mathcal{J}^{i} \otimes \mathcal{J}^{i}
$$

\footnotetext{
${ }^{1}$ The normalization of the generators with respect to the Killing product in $\mathfrak{s u}(2): \kappa(X, Y)=$ $\operatorname{tr}(X Y)$ is such that $\kappa\left(J^{a}, J^{b}\right)=1 / 2$ and correspondingly the root has length squared $\psi=2$.
} 
The volume form reads:

$$
\omega_{[3]}=\mathcal{J}^{1} \wedge \mathcal{J}^{2} \wedge \mathcal{J}^{3} .
$$

In the Euler-angle parameterization, Eq. (B.5) reads (for a radius-L three-sphere):

$$
\mathrm{d} s^{2}=\frac{L^{2}}{4}\left(\mathrm{~d} \alpha^{2}+\mathrm{d} \gamma^{2}+2 \cos \beta \mathrm{d} \alpha \mathrm{d} \gamma+\mathrm{d} \beta^{2}\right)
$$

whereas (B.6) leads to

$$
\omega_{[3]}=\frac{L^{3}}{8} \sin \beta \mathrm{d} \alpha \wedge \mathrm{d} \beta \wedge \mathrm{d} \gamma
$$

The Levi-Civita connection has scalar curvature $R=6 / L^{2}$.

The isometry group of the $S U(2)$ group manifold is generated by left or right actions on $g: g \rightarrow h g$ or $g \rightarrow g h \forall h \in S U(2)$. From the four-dimensional point of view, it is generated by the rotations $\zeta_{a b}=i\left(x_{a} \partial_{b}-x_{b} \partial_{a}\right)$ with $x_{a}=\delta_{a b} x^{b}$. We list here explicitly the six generators, as well as the group action they correspond to:

$$
\begin{array}{rlrl}
L_{1} & =\frac{1}{2}\left(-\zeta_{32}+\zeta_{41}\right), & g & \rightarrow \mathrm{e}^{-i \frac{\lambda}{2} \sigma^{1}} g, \\
L_{2} & =\frac{1}{2}\left(-\zeta_{43}-\zeta_{12}\right), & g & \rightarrow \mathrm{e}^{i \frac{\lambda}{2} \sigma^{2}} g, \\
L_{3} & =\frac{1}{2}\left(-\zeta_{31}-\zeta_{42}\right), & g & \rightarrow \mathrm{e}^{i \frac{\lambda}{2} \sigma^{3}} g, \\
R_{1} & =\frac{1}{2}\left(\zeta_{41}+\zeta_{32}\right), & g & \rightarrow g \mathrm{e}^{i \frac{\lambda}{2} \sigma^{1}}, \\
R_{2} & =\frac{1}{2}\left(-\zeta_{43}+\zeta_{12}\right), & g & \rightarrow g \mathrm{e}^{i \frac{\lambda}{2} \sigma^{2}}, \\
R_{3} & =\frac{1}{2}\left(\zeta_{31}-\zeta_{42}\right), & g \rightarrow g \mathrm{e}^{i \frac{\lambda}{2} \sigma^{3}} .
\end{array}
$$

Both sets satisfy the algebra (B.1). The norms squared of the Killing vectors are all equal to $L^{2} / 4$.

The currents of the $S U(2)_{k}$ WZW model are easily obtained as:

$$
J^{i}=-k \operatorname{tr}\left(\imath \sigma^{i} \partial g g^{-1}\right) \quad \bar{J}^{i}=-k \operatorname{tr}\left(\imath \sigma^{i} g^{-1} \bar{\partial} g\right),
$$

where $L=\sqrt{k}$, at the classical level. Explicit expressions are given in Tab. B.1.

\section{B.2 $\mathrm{AdS}_{3}$}

The commutation relations for the generators of the $S L(2, \mathbb{R})$ algebra are

$$
\left[J^{1}, J^{2}\right]=-\imath J^{3} \quad\left[J^{2}, J^{3}\right]=\imath J^{1} \quad\left[J^{3}, J^{1}\right]=\imath J^{2} .
$$

The sign in the first relation is the only difference with respect to the $S U(2)$ in Eq. (B.1).

The three-dimensional anti-de-Sitter space is the universal covering of the $S L(2, \mathbb{R})$ group manifold. The latter can be embedded in a Lorentzian flat space with signature $(-,+,+,-)$ and coordinates $\left(x^{0}, x^{1}, x^{2}, x^{3}\right)$ :

$$
g=L^{-1}\left(\begin{array}{ll}
x^{0}+x^{2} & x^{1}+x^{3} \\
x^{1}-x^{3} & x^{0}-x^{2}
\end{array}\right)
$$




\begin{tabular}{|c|c|c|}
\hline sector & Killing vector & Current \\
\hline 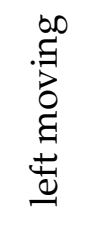 & $\begin{array}{l}\frac{\sin \gamma}{\sin \beta} \partial_{\alpha}+\cos \gamma \partial_{\beta}-\frac{\sin \gamma}{\tan \beta} \partial_{\gamma} \\
\frac{\cos \gamma}{\sin \beta} \partial_{\alpha}-\sin \gamma \partial_{\beta}-\frac{\cos \gamma}{\tan \beta} \partial_{\gamma} \\
\partial_{\gamma}\end{array}$ & $\begin{array}{l}k(\sin \beta \sin \gamma \partial \alpha+\cos \gamma \partial b) \\
k(\cos \gamma \sin \beta \partial \alpha-\sin \gamma \partial \beta) \\
k(\partial \gamma+\cos \beta \partial \alpha)\end{array}$ \\
\hline 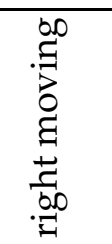 & $\begin{array}{l}-\frac{\sin \alpha}{\tan \beta} \partial_{\alpha}+\cos \alpha \partial_{\beta}+\frac{\sin \alpha}{\sin \beta} \partial_{\gamma} \\
\frac{\cos \alpha}{\tan \beta} \partial_{\alpha}+\sin \alpha \partial_{\beta}-\frac{\cos \alpha}{\sin \beta} \partial_{\gamma} \\
\partial_{\alpha}\end{array}$ & $\begin{array}{l}k(\cos \alpha \bar{\partial} \beta+\sin \alpha \sin \beta \bar{\partial} \gamma) \\
k(\sin \alpha \bar{\partial} \beta-\cos \alpha \sin \beta \bar{\partial} \gamma) \\
k(\bar{\partial} \alpha+\cos \beta \bar{\partial} \gamma)\end{array}$ \\
\hline
\end{tabular}

Table B.1: Killing vectors $\left\{\imath L_{1}, \imath L_{2}, \imath L_{3}\right\}$ and $\left\{\imath R_{1}, \imath R_{2}, \imath R_{3}\right\}$, and holomorphic and anti-holomorphic currents for $S U(2)$ in Euler angles.

where $L$ is the radius of $\mathrm{AdS}_{3}$. On can again introduce Euler-like angles

$$
g=\mathrm{e}^{l(\tau+\phi) \sigma_{2} / 2} \mathrm{e}^{\rho \sigma_{1}} \mathrm{e}^{l(\tau-\phi) \sigma_{2} / 2},
$$

which provide good global coordinates for $\mathrm{AdS}_{3}$ when $\tau \in(-\infty,+\infty), \rho \in[0, \infty)$, and $\phi \in[0,2 \pi)$.

An invariant metric (see Eq. (B.5)) can be introduced on $\mathrm{AdS}_{3}$. In Euler angles, the latter reads:

$$
\mathrm{d} s^{2}=L^{2}\left[-\cosh ^{2} \rho \mathrm{d} \tau^{2}+\mathrm{d} \rho^{2}+\sinh ^{2} \rho \mathrm{d} \phi^{2}\right] .
$$

The Ricci scalar of the corresponding Levi-Civita connection is $R=-6 / L^{2}$.

The isometry group of the $S L(2, \mathbb{R})$ group manifold is generated by left or right actions on $g: g \rightarrow h g$ or $g \rightarrow g h \forall h \in S L(2, \mathbb{R})$. From the four-dimensional point of view, it is generated by the Lorentz boosts or rotations $\zeta_{a b}=i\left(x_{a} \partial_{b}-x_{b} \partial_{a}\right)$ with $x_{a}=\eta_{a b} x^{b}$. We list here explicitly the six generators, as well as the group action they correspond to:

$$
\begin{array}{rlrl}
L_{1} & =\frac{1}{2}\left(\zeta_{32}-\zeta_{01}\right), & g & \rightarrow \mathrm{e}^{-\frac{\lambda}{2} \sigma^{1}} g, \\
L_{2} & =\frac{1}{2}\left(-\zeta_{31}-\zeta_{02}\right), & g & \rightarrow \mathrm{e}^{-\frac{\lambda}{2} \sigma^{3}} g, \\
L_{3} & =\frac{1}{2}\left(\zeta_{03}-\zeta_{12}\right), & g & \rightarrow \mathrm{e}^{i \frac{\lambda}{2} \sigma^{2}} g, \\
R_{1} & =\frac{1}{2}\left(\zeta_{01}+\zeta_{32}\right), & g & \rightarrow g \mathrm{e}^{\frac{\lambda}{2} \sigma^{1}}, \\
R_{2} & =\frac{1}{2}\left(\zeta_{31}-\zeta_{02}\right), & g \rightarrow g \mathrm{e}^{-\frac{\lambda}{2} \sigma^{3}}, \\
R_{3} & =\frac{1}{2}\left(\zeta_{03}+\zeta_{12}\right), & g \rightarrow g \mathrm{e}^{i \frac{\lambda}{2} \sigma^{2}} .
\end{array}
$$

Both sets satisfy the algebra (B.11). The norms of the Killing vectors are the following:

$$
\left\|\imath L_{1}\right\|^{2}=\left\|\imath R_{1}\right\|^{2}=\left\|\imath L_{2}\right\|^{2}=\left\|\imath R_{2}\right\|^{2}=-\left\|\imath L_{3}\right\|^{2}=-\left\|\imath R_{3}\right\|^{2}=\frac{L^{2}}{4} .
$$


Moreover $L_{i} \cdot L_{j}=0$ for $i \neq j$ and similarly for the right set. Left vectors are not orthogonal to right ones.

The isometries of the $S L(2, \mathbb{R})$ group manifold turn into symmetries of the $S L(2, \mathbb{R})_{k}$ WZW model, where they are realized in terms of conserved currents ${ }^{2}$ :

$$
\begin{array}{ll}
J^{1}(z) \pm J^{3}(z)=-k \operatorname{tr}\left(\left(\sigma^{1} \mp \imath \sigma^{2}\right) \partial g g^{-1}\right), & J^{2}(z)=-k \operatorname{tr}\left(\sigma^{3} \partial g g^{-1}\right), \\
\bar{J}^{1}(\bar{z}) \pm \bar{J}^{3}(\bar{z})=k \operatorname{tr}\left(\left(\sigma^{1} \pm \imath \sigma^{2}\right) g^{-1} \bar{\partial} g\right), & \bar{J}^{2}(\bar{z})=-k \operatorname{tr}\left(\sigma^{3} g^{-1} \bar{\partial} g\right) .
\end{array}
$$

At the quantum level, these currents, when properly normalized, satisfy the following affine $S L(2, \mathbb{R})_{k} \mathrm{OPA}^{3}$ :

$$
\begin{aligned}
J^{3}(z) J^{3}(0) & \sim-\frac{k}{2 z^{2}} \\
J^{3}(z) J^{ \pm}(0) & \sim \pm \frac{J^{ \pm}}{z}, \\
J^{+}(z) J^{-}(0) & \sim \frac{2 J^{3}}{z}-\frac{k}{z^{2}}
\end{aligned}
$$

and similarly for the right movers. The central charge of the enveloping Virasoro algebra is $c=3+6 /(k-2)$.

We will introduce three different coordinate systems where the structure of $\mathrm{AdS}_{3}$ as a Hopf fibration is more transparent. They are explicitly described in the following.

- The $(\rho, t, \phi)$ coordinate system used to describe the magnetic deformation is defined as follows:

$$
\left\{\begin{array}{l}
\frac{x_{0}}{L}=\cosh \frac{\rho}{2} \cosh \frac{\phi}{2} \cos \frac{t}{2}-\sinh \frac{\rho}{2} \sinh \frac{\phi}{2} \sin \frac{t}{2} \\
\frac{x_{1}}{L}=-\sinh \frac{\rho}{2} \sinh \frac{\phi}{2} \cos \frac{t}{2}-\cosh \frac{\rho}{2} \sinh \frac{\phi}{2} \sin \frac{t}{2} \\
\frac{x_{2}}{L}=-\cosh \frac{\rho}{2} \sinh \frac{\phi}{2} \cos \frac{t}{2}+\sinh \frac{\rho}{2} \cosh \frac{\phi}{2} \sin \frac{t}{2} \\
\frac{x_{3}}{L}=-\sinh \frac{\rho}{2} \sinh \frac{\phi}{2} \cos \frac{t}{2}-\cosh \frac{\rho}{2} \cosh \frac{\phi}{2} \sin \frac{t}{2}
\end{array}\right.
$$

The metric (B.5) reads:

$$
\mathrm{d} s^{2}=\frac{L^{2}}{4}\left(\mathrm{~d} \rho^{2}+\mathrm{d} \phi^{2}-\mathrm{d} t^{2}-2 \sinh \rho \mathrm{d} t \mathrm{~d} \phi\right)
$$

and the corresponding volume form is:

$$
\omega_{[3]}=\frac{L^{3}}{8} \cosh \rho \cdot \mathrm{d} \rho \wedge \mathrm{d} \phi \wedge \mathrm{d} t
$$

Killing vectors and currents are given in Tab. B.2. It is worth to remark that this coordinate system is such that the $t$-coordinate lines coincide with the integral curves of the Killing vector $\imath L_{3}$, whereas the $\phi$-lines are the curves of $\imath R_{2}$.

\footnotetext{
${ }^{2}$ When writing actions a choice of gauge for the NS potential is implicitly made, which breaks part of the symmetry: boundary terms appear in the transformations. These must be properly taken into account in order to reach the conserved currents. Although the expressions for the latter are not unique, they can be put in an improved-Noether form, in which they have only holomorphic (for $L_{i}{ }^{\prime}$ s) or anti-holomorphic (for $R_{j}$ 's) components.

${ }^{3}$ In some conventions the level is $x=-k$. This allows to unify commutation relations for the affine $S L(2, \mathbb{R})_{x}$ and $S U(2)_{x}$ algebras. Unitarity demands $x<-2$ for the former and $0<x$ with integer $x$ for the latter.
} 
- The $(r, x, \tau)$ coordinate system used to describe the electric deformation is defined as follows:

$$
\left\{\begin{array}{l}
\frac{x_{0}}{L}=\cosh \frac{r}{2} \cosh \frac{x}{2} \cos \frac{\tau}{2}+\sinh \frac{r}{2} \sinh \frac{x}{2} \sin \frac{\tau}{2} \\
\frac{x_{1}}{L}=-\sinh \frac{r}{2} \cosh \frac{x}{2} \cos \frac{\tau}{2}+\cosh \frac{r}{2} \sinh \frac{x}{2} \sin \frac{\tau}{2} \\
\frac{x_{2}}{L}=-\cosh \frac{r}{2} \sinh \frac{x}{2} \cos \frac{\tau}{2}-\sinh \frac{r}{2} \cosh \frac{x}{2} \sin \frac{\tau}{2} \\
\frac{x_{3}}{L}=\sinh \frac{r}{2} \sinh \frac{x}{2} \cos \frac{\tau}{2}-\cosh \frac{r}{2} \cosh \frac{x}{2} \sin \frac{\tau}{2} .
\end{array}\right.
$$

For $\{r, x, \tau\} \in \mathbb{R}^{3}$, this patch covers exactly once the whole $\mathrm{AdS}_{3}$, and is regular everywhere [CH94]. The metric is then given by

$$
\mathrm{d} s^{2}=\frac{L^{2}}{4}\left(\mathrm{~d} r^{2}+\mathrm{d} x^{2}-\mathrm{d} \tau^{2}+2 \sinh r \mathrm{~d} x \mathrm{~d} \tau\right)
$$

and correspondingly the volume form is

$$
\omega_{[3]}=\frac{L^{3}}{8} \cosh r \mathrm{~d} r \wedge \mathrm{d} x \wedge \mathrm{d} \tau .
$$

Killing vectors and currents are given in Tab. B.3. In this case the $x$-coordinate lines coincide with the integral curves of the Killing vector $\imath R_{2}$, whereas the $\tau$-lines are the curves of $\imath R_{3}$.

- The Poincaré coordinate system used to obtain the electromagnetic-wave background is defined by

$$
\begin{cases}x^{0}+x^{2} & =\frac{L}{u} \\ x^{0}-x^{2} & =L u+\frac{L x^{+} x^{-}}{u} \\ x^{1} \pm x^{3} & =\frac{L x^{ \pm}}{u} .\end{cases}
$$

For $\left\{u, x^{+}, x^{-}\right\} \in \mathbb{R}^{3}$, the Poincaré coordinates cover once the $S L(2 \mathbb{R})$ group manifold. Its universal covering, $\mathrm{AdS}_{3}$, requires an infinite number of such patches. Moreover, these coordinates exhibit a Rindler horizon at $|u| \rightarrow \infty$; the conformal boundary is at $|u| \rightarrow 0$. Now the metric reads:

$$
\mathrm{d} s^{2}=\frac{L^{2}}{u^{2}}\left(\mathrm{~d} u^{2}+\mathrm{d} x^{+} \mathrm{d} x^{-}\right)
$$

and the volume form:

$$
\omega_{[3]}=\frac{L^{3}}{2 u^{3}} \mathrm{~d} u \wedge \mathrm{d} x^{-} \wedge \mathrm{d} x^{+} .
$$

In these coordinates it is simple to write certain a linear combination of the Killing vector so to obtain explicitly a light-like isometry generator. For this reason in Tab. B.4 we report the $\left\{L_{1}+L_{3}, L_{1}-L_{3}, L_{2}, R_{1}+R_{3}, R_{1}-R_{3}, R_{2}\right\}$ isometry generators and the corresponding $\left\{J_{1}+J_{3}, J_{1}-J_{3}, J_{2}, \bar{J}_{1}+\bar{J}_{3}, \bar{J}_{1}-\bar{J}_{3}, \bar{J}_{2}\right\}$ currents.

Finally, another useful although not global, set of coordinates is defined by

$$
g=e^{\frac{\psi-\varphi}{2} \sigma^{3}} e^{\imath t \sigma^{1}} e^{\frac{\psi+\varphi}{2} \sigma^{3}},
$$

( $\psi$ and $\varphi$ are not compact coordinates). The metric reads:

$$
\mathrm{d} s^{2}=L^{2}\left[\cos ^{2} t \mathrm{~d} \psi^{2}-\mathrm{d} t^{2}+\sin ^{2} t \mathrm{~d} \varphi^{2}\right],
$$

with volume form

$$
\omega_{[3]}=\frac{L^{3}}{2} \sin 2 t \mathrm{~d} t \wedge \mathrm{d} \psi \wedge \mathrm{d} \varphi .
$$

Now $L_{2}=\frac{1}{2}\left(\partial_{\psi}-\partial_{\varphi}\right)$ and $R_{2}=\frac{1}{2}\left(\partial_{\psi}+\partial_{\varphi}\right)$. 


\begin{tabular}{|c|c|c|}
\hline sector & Killing vector & Current \\
\hline 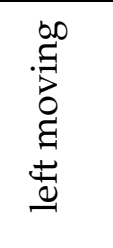 & $\begin{array}{l}\cos t \partial_{\rho}+\frac{\sin t}{\cosh \rho} \partial_{\phi}-\sin t \tanh \rho \partial_{t} \\
-\sin t \partial_{\rho}+\frac{\cos t}{\cosh \rho} \partial_{\phi}-\cos t \tanh \rho \partial_{t} \\
-\partial_{t}\end{array}$ & $\begin{array}{l}k(\cos t \partial \rho+\cosh \rho \sin t \partial \phi) \\
k(\cos t \cosh \rho \partial \phi-\sin t \partial \rho) \\
k(\partial t+\sinh \rho \partial \phi)\end{array}$ \\
\hline 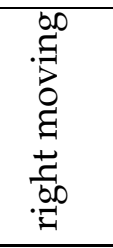 & $\begin{array}{l}\cosh \phi \partial_{\rho}-\sinh \phi \tanh \rho \partial_{\phi}-\frac{\sinh \phi}{\cosh \rho} \partial_{t} \\
\partial_{\phi} \\
\sinh \phi \partial_{\rho}-\cosh \phi \tanh \rho \partial_{\phi}-\frac{\cosh \phi}{\cosh \rho} \partial_{t}\end{array}$ & $\begin{array}{l}-k(\cosh \phi \bar{\partial} \rho+\cosh \rho \sinh \phi \bar{\partial} t) \\
k(\bar{\partial} \phi-\sinh \rho \bar{\partial} t) \\
k(\cosh \rho \cosh \phi \bar{\partial} t+\sinh \phi \bar{\partial} \rho)\end{array}$ \\
\hline
\end{tabular}

Table B.2: Killing vectors $\left\{\imath L_{1}, \imath L_{2}, \imath L_{3}\right\}$ and $\left\{\imath R_{1}, \imath R_{2}, \imath R_{3}\right\}$, and holomorphic and anti-holomorphic currents for the $(\rho, t, \phi)$ coordinate system (elliptic base).

\begin{tabular}{|c|c|c|}
\hline sector & Killing vector & Current \\
\hline 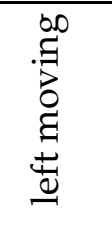 & $\begin{array}{l}\cosh x \partial_{r}-\sinh x \tanh r \partial_{x}+\frac{\sinh x}{\cosh r} \partial_{\tau} \\
\partial_{x} \\
-\sinh x \partial_{r}+\cosh x \tanh r \partial_{x}-\frac{\cosh x}{\cosh r} \partial_{\tau}\end{array}$ & $\begin{array}{l}k(\cosh x \partial r-\cosh r \sinh x \partial \tau) \\
k(\partial x+\sinh r \partial \tau) \\
k(\cosh r \cosh x \partial \tau-\sinh x \partial r)\end{array}$ \\
\hline 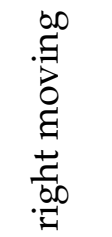 & $\begin{array}{l}-\cos \tau \partial_{r}+\frac{\sin \tau}{\cosh \tau} \partial_{x}-\sin \tau \tanh \tau \partial_{\tau} \\
\frac{(\cos \tau+\sin \tau \tanh r) \partial_{x}+\left(\cos \tau \sinh r-\frac{\sin \tau}{\cosh r}\right) \partial_{\tau}}{\cosh r} \\
-\partial_{\tau}\end{array}$ & $\begin{array}{l}k(-\cos \tau \bar{\partial} r+\cosh r \sin \tau \bar{\partial} r) \\
k(\cos \tau \cosh r \bar{\partial} x+\sin \tau \bar{\partial} r) \\
k(\bar{\partial} \tau-\sinh r \bar{\partial} x)\end{array}$ \\
\hline
\end{tabular}

Table B.3: Killing vectors $\left\{\imath L_{1}, \imath L_{2}, \imath L_{3}\right\}$ and $\left\{\imath R_{1}, \imath R_{2}, \imath R_{3}\right\}$, and holomorphic and anti-holomorphic currents for the $(r, x, \tau)$ coordinate system (hyperbolic base).

\section{B.3 SU (3)}

To obtain the the Cartan-Weyl basis $\left\{H_{a}, E^{\alpha_{j}}\right\}$ for the $\mathfrak{s u}(3)$ algebra we need to choose the positive roots as follows:

$$
\alpha_{1}=[\sqrt{2}, 0] \quad \alpha_{2}=[-1 / \sqrt{2}, \sqrt{3 / 2}] \quad \alpha_{3}=[1 / \sqrt{2}, \sqrt{3 / 2}]
$$

The usual choice for the defining representation is:

$$
\begin{array}{ccc}
H_{1}=\frac{1}{\sqrt{2}}\left(\begin{array}{ccc}
1 & 0 & 0 \\
0 & -1 & 0 \\
0 & 0 & 0
\end{array}\right) & H_{2}=\frac{1}{\sqrt{6}}\left(\begin{array}{ccc}
1 & 0 & 0 \\
0 & 1 & 0 \\
0 & 0 & -2
\end{array}\right) \\
E_{2}^{+}=\left(\begin{array}{lll}
0 & 0 & 0 \\
0 & 0 & 1 \\
0 & 0 & 0
\end{array}\right) & E_{3}^{+}=\left(\begin{array}{ccc}
0 & 0 & 1 \\
0 & 0 & 0 \\
0 & 0 & 0
\end{array}\right) &
\end{array}
$$

and $E_{j}^{-}=\left(E_{j}^{+}\right)^{t}$. 


\begin{tabular}{|c|c|c|}
\hline sector & Killing vector & Current \\
\hline 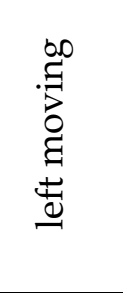 & $\begin{array}{l}-\partial_{-} \\
u x^{-} \partial_{u}-u^{2} \partial_{+}+\left(x^{-}\right)^{2} \partial_{-} \\
\frac{u}{2} \partial_{u}+x^{-} \partial_{-}\end{array}$ & $\begin{array}{l}-2 k \frac{\partial x^{+}}{u^{2}} \\
2 k\left(2 x^{-} \frac{\partial u}{u}-\partial x^{-}+\left(x^{-}\right)^{2} \frac{\partial x^{+}}{u^{2}}\right) \\
2 k\left(\frac{\partial u}{u}+x^{-} \frac{\partial x^{+}}{u^{2}}\right)\end{array}$ \\
\hline 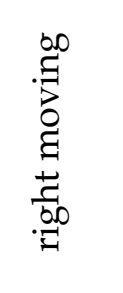 & $\begin{array}{l}\partial_{+} \\
-u x^{+} \partial_{u}-\left(x^{+}\right)^{2} \partial_{+}+u^{2} \partial_{-} \\
\frac{u}{2} \partial_{u}+x^{+} \partial_{+}\end{array}$ & $\begin{array}{l}2 k \frac{\bar{\partial} x^{-}}{u^{2}} \\
2 k\left(-2 x^{+} \frac{\bar{\partial} u}{u}+\bar{\partial} x^{+}-\left(x^{+}\right)^{2} \frac{\bar{\partial} x^{-}}{u^{2}}\right) \\
2 k\left(\frac{\bar{\partial} u}{u}+x^{+} \frac{\bar{\partial} x^{-}}{u^{2}}\right)\end{array}$ \\
\hline
\end{tabular}

Table B.4: Killing vectors, and holomorphic and anti-holomorphic currents in Poincaré coordinates (parabolic base). The $\left\{\imath L_{1}+\imath L_{3}, \imath L_{1}-\imath L_{3}, \imath L_{2}, \imath R_{1}+\right.$ $\left.\imath R_{3}, \imath R_{1}-\imath R_{3}, \imath R_{2}\right\}$ isometry generators and the corresponding $\left\{J_{1}+J_{3}, J_{1}-\right.$ $\left.J_{3}, J_{2}, \bar{J}_{1}+\bar{J}_{3}, \bar{J}_{1}-\bar{J}_{3}, \bar{J}_{2}\right\}$ currents are represented so to explicitly obtain lightlike isometry generators.

A good parametrisation for the $S U$ (3) group can be obtained via the Gauss decomposition: every matrix $g \in S U(3)$ is written as the product:

$$
g=b_{-} d b_{+}
$$

where $b_{-}$is a lower triangular matrix with unit diagonal elements, $b_{+}$is a upper triangular matrix with unit diagonal elements and $d$ is a diagonal matrix with unit determinant. The element $g$ is written as:

$$
\begin{aligned}
& g\left(z_{1}, z_{2}, z_{3}, \psi_{1}, \psi_{2}\right)=\exp \left[z_{1} E_{1}^{-}+z_{2} E_{3}^{-}+\left(z_{3}-\frac{z_{1} z_{2}}{2}\right) E_{2}^{-}\right] \times \\
& \quad \times e^{-F_{1} H_{1}-F_{2} H_{2}} \exp \left[\bar{w}_{1} E_{1}^{+}+\bar{w}_{2} E_{3}^{+}+\left(\bar{w}_{3}-\frac{\bar{w} 1 \bar{w}_{2}}{2}\right) E_{2}^{+}\right] e^{\imath \psi_{1} H_{1}+\imath \psi_{2} H_{2}}
\end{aligned}
$$

where $z_{\mu}$ are 3 complex parameters, $\psi_{i}$ are two real and $F_{1}$ and $F_{2}$ are positive real functions of the $z_{\mu}$ 's:

$$
\left\{\begin{array}{l}
F_{1}=\log f_{1}=\log \left(1+\left|z_{1}\right|^{2}+\left|z_{3}\right|^{2}\right) \\
F_{2}=\log f_{2}=\log \left(1+\left|z_{2}\right|^{2}+\left|z_{3}-z_{1} z_{2}\right|^{2}\right)
\end{array}\right.
$$

By imposing $g\left(z_{\mu}, \psi_{a}\right)$ to be unitary we find that the $w_{\mu}$ 's are complex functions of the $z_{\mu}$ 's:

$$
\left\{\begin{array}{l}
w_{1}=-\frac{z_{1}+\bar{z}_{2} z_{3}}{\sqrt{f 2}} \\
w_{2}=\frac{z_{1} z_{3}-z_{2}\left(1+\left|z_{1}\right|^{2}\right)}{\sqrt{f_{1}}} \\
w_{3}=-\left(z_{3}-z_{1} z_{2}\right) \sqrt{\frac{f_{1}}{f_{2}}}
\end{array}\right.
$$


and the defining element $g\left(z_{\mu}, \psi_{a}\right)$ can then be written explicitly as:

$$
\begin{aligned}
g\left(z_{1}, z_{2}, z_{3}, \psi_{1}, \psi_{2}\right)= & \left(\begin{array}{ccc}
1 & 0 & 0 \\
z_{1} & 1 & 0 \\
z_{3} & z_{2} & 1
\end{array}\right)\left(\begin{array}{ccc}
\frac{1}{\sqrt{f_{1}}} & 0 & 0 \\
0 & \sqrt{f_{1} / f_{2}} & 0 \\
0 & 0 & \sqrt{f_{2}}
\end{array}\right) \times \\
& \times\left(\begin{array}{ccc}
1 & \bar{w}_{1} & \bar{w}_{3} \\
0 & 1 & \bar{w}_{2} \\
0 & 0 & 1
\end{array}\right)\left(\begin{array}{ccc}
e^{\imath \psi_{1} / 2} & 0 & 0 \\
0 & e^{-\imath\left(\psi_{1}-\psi_{2}\right) / 2} & 0 \\
0 & 0 & e^{\imath \psi_{2} / 2}
\end{array}\right)
\end{aligned}
$$

Now, to build a metric for the tangent space to $S U$ (3) we can define the 1-form $\Omega(\mathbf{z},-)=g^{-1}(\mathbf{z},-) \mathrm{d} g(\mathbf{z},-)$ and write the Killing-Cartan metric tensor as $g_{\mathrm{KC}}=\operatorname{tr}\left(\Omega^{\dagger} \Omega\right)=$ $-\operatorname{tr}(\Omega \Omega)$ where we have used explicitly the property of anti-Hermiticity of $\Omega$ (that lives in the $\mathfrak{s u}$ (3) algebra). The explicit calculation is lengthy but straightforward. The main advantage of this parametrization from our point of view is that it allows for a "natural" embedding of the $S U$ (3) /U (1) ${ }^{2}$ coset (see e.g. [GK98] or [KT00]): in fact in these coordinates the Kähler potential is

$$
\begin{aligned}
& K\left(z_{\mu}, \bar{z}_{\mu}\right)=\log \left(f_{1}\left(z_{\mu}\right) f_{2}\left(z_{\mu}\right)\right)= \\
& \quad=\log \left[\left(1+\left|z_{1}\right|^{2}+\left|z_{3}\right|^{2}\right)\left(1+\left|z_{2}\right|^{2}+\left|z_{3}-z_{1} z_{2}\right|^{2}\right)\right]
\end{aligned}
$$

and the coset Kähler metric is hence simply obtained as:

$$
g_{\alpha \bar{\beta}} \mathrm{d} z^{\alpha} \otimes \mathrm{d} \bar{z}^{\beta}=\frac{\partial^{2}}{\partial z_{\alpha} \partial \bar{z}_{\beta}} K\left(z_{\mu}, \bar{z}_{\mu}\right) \mathrm{d} z^{\alpha} \otimes \mathrm{d} \bar{z}^{\beta}
$$

Another commonly used $\mathfrak{s u}$ (3) basis is given by the Gell-Mann matrices:

$$
\begin{aligned}
& \gamma_{1}=\frac{1}{\sqrt{2}}\left(\begin{array}{lll}
0 & \imath & 0 \\
\imath & 0 & 0 \\
0 & 0 & 0
\end{array}\right) \quad \gamma_{2}=\frac{1}{\sqrt{2}}\left(\begin{array}{ccc}
0 & 1 & 0 \\
-1 & 0 & 0 \\
0 & 0 & 0
\end{array}\right) \quad \gamma_{3}=\frac{1}{\sqrt{2}}\left(\begin{array}{ccc}
\imath & 0 & 0 \\
0 & -\imath & 0 \\
0 & 0 & 0
\end{array}\right) \\
& \gamma_{4}=\frac{1}{\sqrt{2}}\left(\begin{array}{lll}
0 & 0 & \imath \\
0 & 0 & 0 \\
\imath & 0 & 0
\end{array}\right) \quad \gamma_{5}=\frac{1}{\sqrt{2}}\left(\begin{array}{ccc}
0 & 0 & 1 \\
0 & 0 & 0 \\
-1 & 0 & 0
\end{array}\right) \quad \gamma_{6}=\frac{1}{\sqrt{2}}\left(\begin{array}{lll}
0 & 0 & 0 \\
0 & 0 & \imath \\
0 & \imath & 0
\end{array}\right) \\
& \gamma_{7}=\frac{1}{\sqrt{2}}\left(\begin{array}{ccc}
0 & 0 & 0 \\
0 & 0 & 1 \\
0 & -1 & 0
\end{array}\right) \quad \gamma_{8}=\frac{1}{\sqrt{6}}\left(\begin{array}{ccc}
1 & 0 & 0 \\
0 & \imath & 0 \\
0 & 0 & -2 \imath
\end{array}\right)
\end{aligned}
$$

which presents the advantage of being orthonormal $\kappa\left(\lambda_{i}, \lambda_{j}\right)=\delta_{i j}$. In this case the Cartan subalgebra is generated by $\mathfrak{k}=\left\langle\lambda_{3}, \lambda_{8}\right\rangle$.

\section{B.4 USp (4)}

The symplectic group $S p(4, \mathbb{C})$ is the set of $4 \times 4$ complex matrices that preserve the symplectic form $J$ :

$$
J=\left(\begin{array}{c|c}
0 & \mathbb{I}_{2 \times 2} \\
\hline-\mathbb{I}_{2 \times 2} & 0
\end{array}\right)
$$

that is

$$
S p(4, \mathbb{C})=\left\{g \mid g^{t} J g=J\right\}
$$


The unitary symplectic group $U S p(4, \mathbb{C})$ is the compact group obtained as the intersection of $S p(4, \mathbb{C})$ with $U(4)$ :

$$
U S p(4, \mathbb{C})=S p(4, \mathbb{C}) \cap U(4)
$$

It follows easily that the Lie algebra usp (4) is the set of complex matrices $X$ such that:

$$
\mathfrak{u} \mathfrak{p} p(4)=\left\{X \mid X^{t} J+J X^{t}=0\right\}
$$

To obtain the the Cartan-Weyl basis $\left\{H_{a}, E^{\alpha_{j}}\right\}$ we need to choose the positive roots

$$
\alpha_{1}=[\sqrt{2} / 2,-\sqrt{2} / 2] \quad \alpha_{2}=[0, \sqrt{2}] \quad \alpha_{3}=[\sqrt{2} / 2, \sqrt{2} / 2] \quad \alpha_{4}=[\sqrt{2}, 0]
$$

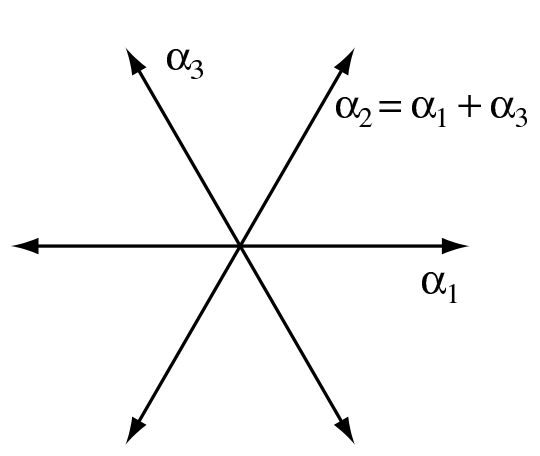

(a) $\operatorname{SU}(2)$

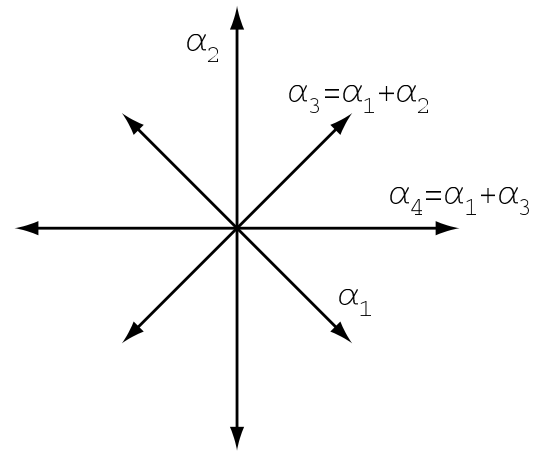

(b) $U S p(4)$

Figure B.1: Root system for $\mathfrak{s u}(3)$ and $\mathfrak{s p}(4)$.

and the $N_{\mu, \nu}$ coefficients:

$$
N_{1,2}=1 \quad N_{1,3}=2
$$

The defining realization is given by the following choice:

$$
\begin{array}{ccc}
H_{1}=\frac{1}{\sqrt{2}}\left(\begin{array}{cccc}
1 & 0 & 0 & 0 \\
0 & 0 & 0 & 0 \\
0 & 0 & -1 & 0 \\
0 & 0 & 0 & 0
\end{array}\right) & H_{2}=\frac{1}{\sqrt{2}}\left(\begin{array}{cccc}
0 & 0 & 0 & 0 \\
0 & 1 & 0 & 0 \\
0 & 0 & 0 & 0 \\
0 & 0 & 0 & -1
\end{array}\right) & E_{1}^{+}=\left(\begin{array}{cccc}
0 & 1 & 0 & 0 \\
0 & 0 & 0 & 0 \\
0 & 0 & 0 & 0 \\
0 & 0 & -1 & 0
\end{array}\right) \\
E_{2}^{+}=\left(\begin{array}{cccc}
0 & 0 & 0 & 0 \\
0 & 0 & 0 & 1 \\
0 & 0 & 0 & 0 \\
0 & 0 & 0 & 0
\end{array}\right) & E_{3}^{+}=\left(\begin{array}{cccc}
0 & 0 & 0 & 1 \\
0 & 0 & 1 & 0 \\
0 & 0 & 0 & 0 \\
0 & 0 & 0 & 0
\end{array}\right) & E_{4}^{+}=\left(\begin{array}{cccc}
0 & 0 & 1 & 0 \\
0 & 0 & 0 & 0 \\
0 & 0 & 0 & 0 \\
0 & 0 & 0 & 0
\end{array}\right)
\end{array}
$$

and $E^{-\alpha_{\mu}}=\left(E^{\alpha_{\mu}}\right)^{t}$ 
Just like in the case of $S U(3)$, the general element in $U S p(4)$ is written as:

$$
\begin{aligned}
& g\left(\gamma_{\mu}, \psi_{a}\right)=\exp \left[\gamma_{1} E_{1}^{-}+\frac{\gamma_{2}}{\sqrt{2}} E_{2}^{-}+\frac{2 \gamma_{3}-\gamma_{1} \gamma_{2}}{2} E_{3}^{-}+\frac{\gamma_{1}^{2} \gamma_{2}-\gamma_{1} \gamma_{3}+\gamma_{4}}{\sqrt{2}} E_{4}^{-}\right] e^{-F_{1} H_{1}-F_{2} H_{2}} \\
& \exp \left[\bar{\beta}_{1} E_{1}^{+}+\frac{\bar{\beta}_{2}}{\sqrt{2}} E_{2}^{+}+\frac{2 \bar{\beta}_{3}-\bar{\beta}_{1} \bar{\beta}_{2}}{2} E_{3}^{+}+\frac{\bar{\beta}_{1}^{2} \bar{\beta}_{2}-\bar{\beta}_{1} \bar{\beta}_{3}+\bar{\beta}_{4}}{\sqrt{2}} E_{4}^{+}\right] e^{\imath \psi_{1} H_{1}+\imath \psi_{2} H_{2}}= \\
& =\left(\begin{array}{cccc}
1 & 0 & 0 & 0 \\
\gamma_{1} & 1 & 0 & 0 \\
\gamma_{4} & -\gamma_{1} \gamma_{2}+\gamma_{3} & 1 & -\gamma_{1} \\
\gamma_{3} & \gamma_{2} & 0 & 1
\end{array}\right)\left(\begin{array}{cccc}
f_{1} & 0 & 0 & 0 \\
0 & f_{2} & 0 & 0 \\
0 & 0 & 1 / f_{1} & 0 \\
0 & 0 & 0 & 1 / f_{2}
\end{array}\right)\left(\begin{array}{cccc}
1 & \bar{\beta}_{1} & \bar{\beta}_{4} & \bar{\beta}_{3} \\
0 & 1 & -\bar{\beta}_{1} \bar{\beta}_{2}+\bar{\beta}_{3} & \bar{\beta}_{2} \\
0 & 0 & 1 & 0 \\
0 & 0 & -\bar{\beta}_{1} & 1
\end{array}\right) \times \\
& \times\left(\begin{array}{cccc}
e^{\imath \psi_{1}} & 0 & 0 & 0 \\
0 & e^{\imath \psi_{2}} & 0 & 0 \\
0 & 0 & e^{-\imath \psi_{1}} & 0 \\
0 & 0 & 0 & e^{-\imath \psi_{2}}
\end{array}\right)
\end{aligned}
$$

A orthonormal basis for the $\mathfrak{u s p}(4)$, similar to the Gell-Mann matrices system is given by the following set of matrices:

$$
\begin{aligned}
& T_{1}=\frac{1}{\sqrt{2}}\left(\begin{array}{cccc}
\imath & 0 & 0 & 0 \\
0 & 0 & 0 & 0 \\
0 & 0 & -\imath & 0 \\
0 & 0 & 0 & 0
\end{array}\right) \quad T_{2}=\frac{1}{\sqrt{2}}\left(\begin{array}{cccc}
0 & 0 & 0 & 0 \\
0 & \imath & 0 & 0 \\
0 & 0 & 0 & 0 \\
0 & 0 & 0 & -\imath
\end{array}\right) \quad T_{3}=\frac{1}{2}\left(\begin{array}{cccc}
0 & \imath & 0 & 0 \\
\imath & 0 & 0 & 0 \\
0 & 0 & 0 & -\imath \\
0 & 0 & -\imath & 0
\end{array}\right) \\
& T_{4}=\frac{1}{2}\left(\begin{array}{cccc}
0 & 1 & 0 & 0 \\
-1 & 0 & 0 & 0 \\
0 & 0 & 0 & 1 \\
0 & 0 & -1 & 0
\end{array}\right) \quad T_{5}=\frac{1}{\sqrt{2}}\left(\begin{array}{cccc}
0 & 0 & 0 & 0 \\
0 & 0 & 0 & \imath \\
0 & 0 & 0 & 0 \\
0 & 1 & 0 & 0
\end{array}\right) \quad T_{6}=\frac{1}{\sqrt{2}}\left(\begin{array}{cccc}
0 & 0 & 0 & 0 \\
0 & 0 & 0 & 1 \\
0 & 0 & 0 & 0 \\
0 & -1 & 0 & 0
\end{array}\right) \\
& T_{7}=\frac{1}{2}\left(\begin{array}{llll}
0 & 0 & 0 & \imath \\
0 & 0 & \imath & 0 \\
0 & \imath & 0 & 0 \\
\imath & 0 & 0 & 0
\end{array}\right) \quad T_{8}=\frac{1}{2}\left(\begin{array}{cccc}
0 & 0 & 0 & 1 \\
0 & 0 & 1 & 0 \\
0 & -1 & 0 & 0 \\
-1 & 0 & 0 & 0
\end{array}\right) \quad T_{9}=\frac{1}{\sqrt{2}}\left(\begin{array}{cccc}
0 & 0 & 1 & 0 \\
0 & 0 & 0 & 0 \\
1 & 0 & 0 & 0 \\
0 & 0 & 0 & 0
\end{array}\right) \\
& T_{10}=\frac{1}{\sqrt{2}}\left(\begin{array}{cccc}
0 & 0 & 1 & 0 \\
0 & 0 & 0 & 0 \\
-1 & 0 & 0 & 0 \\
0 & 0 & 0 & 0
\end{array}\right)
\end{aligned}
$$




\section{APPENDix $C$}

\section{Symmetric deformations of $S L(2, \mathbb{R})$}

The group manifold of $S L(2, \mathbb{R})$ is anti de Sitter in three dimensions. Metric and antisymmetric tensor read (in Euler coordinates, see App. B):

$$
\begin{aligned}
\mathrm{d} s^{2} & =L^{2}\left[\mathrm{~d} \rho^{2}+\sinh ^{2} \rho \mathrm{d} \phi^{2}-\cosh ^{2} \rho \mathrm{d} \tau^{2}\right], \\
H_{[3]} & =L^{2} \sinh 2 \rho \mathrm{d} \rho \wedge \mathrm{d} \phi \wedge \mathrm{d} \tau,
\end{aligned}
$$

with $L$ related to the level of $S L(2, \mathbb{R})_{k}$ as usual: $L=\sqrt{k+2}$. In the case at hand, three different lines of symmetric deformations arise due to the presence of time-like $\left(J^{3}, \bar{J}^{3}\right)$, space-like $\left(J^{1}, \bar{J}^{1}, J^{2}, \bar{J}^{2}\right)$, or null generators [FR03, Fö4, IKP03]. The residual isometry is $U(1) \times U(1)$ that can be time-like $\left(L_{3}, R_{3}\right)$, space-like $\left(L_{2}, R_{2}\right)$ or null $\left(L_{1}+L_{3}, R_{1}+R_{3}\right)$ depending on the deformation under consideration.

The elliptic deformation is driven by the $J^{3} \bar{J}^{3}$ bilinear. At first order in $\alpha^{\prime}$ the background fields are given $b^{1}$ :

$$
\begin{aligned}
\mathrm{d} s^{2} & =k\left[\mathrm{~d} \rho^{2}+\frac{\sinh ^{2} \rho \mathrm{d} \phi^{2}-\kappa_{3}^{2} \cosh ^{2} \rho \mathrm{d} \tau^{2}}{\Theta_{\kappa_{3}}(\rho)}\right], \\
H_{[3]} & =k \frac{\kappa_{3}^{2} \sinh 2 \rho}{\Theta_{\kappa_{3}}(\rho)^{2}} \mathrm{~d} \rho \wedge \mathrm{d} \phi \wedge \mathrm{d} \tau, \\
\mathrm{e}^{\Phi} & =\frac{\Theta_{\kappa_{3}}(\rho)}{\kappa_{3}} .
\end{aligned}
$$

where $\Theta_{\kappa_{3}}(\rho)=\cosh ^{2} \rho-\kappa_{3} \sinh ^{2} \rho$. At extreme deformation $\left(\kappa_{3}^{2} \rightarrow 0\right)$, a time-like direction decouples and we are left with the axial ${ }^{2} S L(2, \mathbb{R})_{k} / U(1)_{\text {time. }}$. The target space of the latter is the cigar geometry (also called Euclidean two-dimensional black hole):

$$
\begin{aligned}
\mathrm{e}^{\Phi} & \sim \cosh ^{2} \rho, \\
\mathrm{d} s^{2} & =k\left[\mathrm{~d} \rho^{2}+\tanh ^{2} \rho \mathrm{d} \phi^{2}\right],
\end{aligned}
$$

$(0 \leq \rho<\infty$ and $0 \leq \phi \leq 2 \pi)$.

\footnotetext{
${ }^{1}$ The extra index " 3 " in the deformation parameter $\kappa$ reminds that the deformation refers here to $J^{3} \bar{J}^{3}$.

${ }^{2}$ The deformation parameter has two T-dual branches. The extreme values of deformation correspond to the axial or vector gaugings. The vector gauging leads to the trumpet. For the $S U(2)_{k} / U(1)$, both gaugings correspond to the bell.
} 
Similarly, with $J^{2} \bar{J}^{2}$ one generates the hyperbolic deformation. This allows to reach the Lorentzian two-dimensional black hole times a free space-like line. Using the coordinates defined in Eq. (B.28), we find:

$$
\begin{aligned}
\mathrm{d} s^{2} & =k\left[-\mathrm{d} t^{2}+\frac{\sin ^{2} t \mathrm{~d} \varphi^{2}+\kappa_{2}^{2} \cos ^{2} t \mathrm{~d} \psi^{2}}{\Delta_{\kappa_{2}}(t)}\right], \\
H_{[3]} & =k \frac{\kappa_{2}^{2} \sin 2 t}{\Delta_{\kappa_{2}}(t)^{2}} \mathrm{~d} t \wedge \mathrm{d} \psi \wedge \mathrm{d} \phi, \\
\mathrm{e}^{\Phi} & =\frac{\Delta_{\kappa_{2}}(t)}{\kappa_{2}},
\end{aligned}
$$

where $\Delta_{\kappa_{2}}(t)=\cos ^{2} t+\kappa_{2}^{2} \sin ^{2} t$. This coordinate patch does not cover the full $\mathrm{AdS}_{3}$. We will expand on this line in Sec. 4.4.

Finally, the bilinear $\left(J^{1}+J^{3}\right)\left(\bar{J}^{1}+\bar{J}^{3}\right)$ generates the parabolic deformation. Using Poincaré coordinates in Eq. (B.26) $)^{3}$ we obtain:

$$
\begin{aligned}
\mathrm{d} s^{2} & =k\left[\frac{\mathrm{d} u^{2}}{u^{2}}+\frac{\mathrm{d} X^{2}-\mathrm{d} T^{2}}{u^{2}+1 / v}\right], \\
H_{[3]} & =k \frac{2 u}{\left(u^{2}+1 / v\right)^{2}} \mathrm{~d} u \wedge \mathrm{d} T \wedge \mathrm{d} X, \\
\mathrm{e}^{\Phi} & =\frac{u^{2}+1 / v}{u^{2}} .
\end{aligned}
$$

The deformation parameter is $1 / v$. At infinite value of the parameter $v$, we recover pure $\mathrm{AdS}_{3}$; for $v \rightarrow 0$, a whole light-cone decouples and we are left with a single direction and a dilaton field, linear in this direction.

The physical interpretation of the parabolic deformation is far reaching, when $\mathrm{AdS}_{3}$ is considered in the framework of the NS5 / F1 near-horizon background, $\mathrm{AdS}_{3} \times$ $S^{3} \times T^{4}$. In this physical set-up, the parameter $v$ is the density of F1's (number of fundamental strings over the volume of the four-torus $T^{4}$ ) [IKP03, KKPR03 $]^{4}$. At infinite density, the background is indeed $\mathrm{AdS}_{3} \times S^{3} \times T^{4}$. At null density, the geometry becomes $\mathbb{R}^{1,2} \times S^{3} \times T^{4}$ plus a linear dilaton and a three-form on the $S^{3}$.

\footnotetext{
${ }^{3}$ Note that $x^{ \pm}=X \pm T$.

${ }^{4}$ Our present convention for the normalization of the dilaton results from Eq. (3.15b). It differs by a factor -2 with respect to the one used in those papers.
} 


\section{Appendix D}

\section{Spectrum of the $S L(2, \mathbb{R})$ super-WZW \\ model}

In this appendix we give a reminder of the superconformal WZW model on $S L(2, \mathbb{R})_{k}$ (for a recent discussion see [GKPS03]). The affine extension of the $\mathfrak{s l}(2, \mathbb{R})$ algebra at level $k$ is obtained by considering two sets of holomorphic and anti-holomorphic currents of dimension one, defined as

$$
J^{\mathrm{M}}(z)=k\left\langle T^{\mathrm{M}}, \operatorname{Ad}_{g} g^{-1} \partial g\right\rangle, \quad \bar{J}^{\mathrm{M}}(\bar{z})=k\left\langle T^{\mathrm{M}}, g^{-1} \bar{\partial} g\right\rangle,
$$

where $\langle\cdot, \cdot\rangle$ is the scalar product (Killing form) in $\mathfrak{s l}(2, \mathbb{R}),\left\{T^{\mathrm{M}}\right\}$ is a set of generators of the algebra that for concreteness we can choose as follows:

$$
T^{1}=\sigma^{1}, \quad T^{2}=\sigma^{3}, \quad T^{3}=\sigma^{2} .
$$

Each set satisfies the OPE

$$
J^{\mathrm{M}}(z) J^{\mathrm{N}}(w) \sim \frac{k \delta^{\mathrm{MN}}}{2(z-w)^{2}}+\frac{f_{\mathrm{P}}^{\mathrm{MN}} J^{\mathrm{P}}(w)}{z-w},
$$

where $f_{\mathrm{P}}^{\mathrm{MN}}$ are the structure constants of the $\mathfrak{s l}(2, \mathbb{R})$ algebra. The chiral algebra contains the Virasoro operator (stress tensor) obtained by the usual Sugawara construction:

$$
T(z)=\sum_{\mathrm{M}} \frac{: J^{\mathrm{M}} J^{\mathrm{M}}:}{k-2} .
$$

A heterotic model is built if we consider a left-moving $\mathcal{N}=1$ extension, obtained by adding 3 free fermions which transform in the adjoint representation. More explicitly:

$$
\begin{aligned}
& T(z)=\sum_{\mathrm{M}} \frac{: J^{\mathrm{M}} J^{\mathrm{M}}:}{k-2}+: \psi_{\mathrm{M}} \partial \psi_{\mathrm{M}}: \\
& G(z)=\frac{2}{k}\left(\sum_{\mathrm{M}} J^{\mathrm{M}} \psi_{\mathrm{M}}-\frac{\imath}{3 k} \sum_{\mathrm{MNP}} f^{\mathrm{MNP}}: \psi_{\mathrm{M}} \psi_{\mathrm{N}} \psi_{\mathrm{P}}:\right) .
\end{aligned}
$$

On the right side, instead of superpartners, we add a right-moving current with total central charge $c=16$. 
Let us focus on the left-moving part. The supercurrents are given by $\psi_{\mathrm{M}}+\theta \sqrt{2 / k} \mathcal{J}_{\mathrm{M}}$ where:

$$
\mathcal{J}_{\mathrm{M}}=J^{\mathrm{M}}-\frac{l}{2} \sum_{\mathrm{NP}} \epsilon^{\mathrm{MNP}} \psi_{\mathrm{N}} \psi_{\mathrm{P}}
$$

it should be noted that the bosonic $J^{\mathrm{M}}$ currents generate an affine $\mathfrak{s l}(2, \mathbb{R})$ algebra at level $k+2$, while the level for the total $\mathcal{J}_{\mathrm{M}}$ currents is $k$.

Let us now single out the operator that we used for both the deformation (Eqs. (4.87)) and the identifications (Sec. 4.4):

$$
\mathcal{J}_{2}=J^{2}+\imath \psi_{1} \psi_{3} \text {. }
$$

Let us now bosonize these currents as follows:

$$
\begin{aligned}
\mathcal{J}_{2} & =-\sqrt{\frac{k}{2} \partial \vartheta_{2},} \\
J^{2} & =-\sqrt{\frac{k+2}{2}} \partial \theta_{2}, \\
\psi_{1} \psi_{3} & =\partial H,
\end{aligned}
$$

and introduce a fourth free boson $X$ so to separate the $\vartheta_{2}$ components both in $\theta_{2}$ and $H$ :

$$
\begin{aligned}
\imath H & =\sqrt{\frac{2}{k}} \vartheta_{2}+\imath \sqrt{\frac{k+2}{k}} X, \\
\theta_{2} & =\sqrt{\frac{2}{k}}\left(\sqrt{\frac{k+2}{2}} \vartheta_{2}+\imath X\right) .
\end{aligned}
$$

A primary field $\Phi_{j \mu \tilde{\mu}}$ of the bosonic $S L(2, \mathbb{R})_{k+2}$ with eigenvalue $\mu$ with respect to $J^{2}$ and $\bar{\mu}$ with respect to $\bar{J}^{2}$ obeys by definition

$$
\begin{aligned}
J^{2}(z) \Phi_{j \mu \bar{\mu}}(w, \bar{w}) & \sim \frac{\mu \Phi_{j \mu \bar{\mu}}(w, \bar{w})}{z-w}, \\
\bar{J}^{2}(\bar{z}) \Phi_{j \mu \bar{\mu}}(w, \bar{w}) & \sim \frac{\bar{\mu} \Phi_{j \mu \bar{\mu}}(w, \bar{w})}{\bar{z}-\bar{w}} .
\end{aligned}
$$

Since $\Phi_{j \mu \bar{\mu}}$ is purely bosonic, the same relation holds for the supercurrent:

$$
\mathcal{J}_{2}(z) \Phi_{j \mu \bar{\mu}}(w, \bar{w}) \sim \frac{\mu \Phi_{j \mu \bar{\mu}}(w, \bar{w})}{z-w} .
$$

Consider now the holomorphic part of $\Phi_{j \mu \bar{\mu}}(z, \bar{z})$. If $\Phi_{j \mu}$ is viewed as a primary in the SWZW model, we can use the parafermion decomposition as follows:

$$
\Phi_{j \mu}(z)=U_{j \mu}(z) e^{\imath \mu \sqrt{2 / k} \vartheta_{2}},
$$

where $U_{j \mu}(z)$ is a primary of the superconformal $S L(2, \mathbb{R})_{k} / U(1)$. On the other hand, we can just consider the bosonic WZW and write:

$$
\Phi_{j \mu}(z)=V_{j \mu}(z) e^{\imath \mu \sqrt{2 /(k+2)} \theta_{2}}=V_{j \mu}(z) e^{\imath \frac{2 m}{k+2} \sqrt{\frac{k+2}{k}} X+\imath \mu \sqrt{2 / k} \vartheta_{2}},
$$

where now $V_{j \mu}(z)$ is a primary of the bosonic $S L(2, \mathbb{R})_{k+2} / U(1)$. The scaling dimension for this latter operator (i.e. its eigenvalue with respect to $L_{0}$ ) is then given by:

$$
\Delta\left(V_{j \mu}\right)=-\frac{j(j+1)}{k}-\frac{\mu^{2}}{k+2} .
$$


An operator in the full supersymmetric $S L(2, \mathbb{R})_{k}$ theory is then obtained by adding the $\psi^{1} \psi^{3}$ fermionic superpartner contribution:

$$
\Phi_{j \mu \nu}(z)=\Phi_{j \mu}(z) e^{\imath v H}=V_{j \mu}(z) e^{\imath\left(\frac{2 \mu}{k+2}+v\right) \sqrt{\frac{k+2}{k}} X} e^{\imath \sqrt{2 / k}(\mu+v) \vartheta_{2}}
$$

that is an eigenvector of $\mathcal{J}_{2}$ with eigenvalue $\mu+v$ where $\mu \in \mathbb{R}$ and $v$ can be decomposed as $v=n+a / 2$ with $n \in \mathbb{N}$ and $a \in \mathbb{Z}_{2}$ depending on whether we consider the NS or R sector. The resulting spectrum can be read directly as:

$$
\begin{array}{r}
\Delta\left(\Phi_{j \mu n}(z)\right)=-\frac{j(j+1)}{k}-\frac{\mu^{2}}{k+2}-\frac{k+2}{2 k}\left(\frac{2 \mu}{k+2}+n+\frac{a}{2}\right)^{2}+\frac{1}{k}\left(\mu+n+\frac{a}{2}\right)^{2}= \\
=-\frac{j(j+1)}{k}-\frac{1}{2}\left(n+\frac{a}{2}\right)^{2} .
\end{array}
$$

Of course the last expression was to be expected since it is the sum of the $\mathfrak{s l}(2, \mathbb{R})_{k+2}$ Casimir and the contribution of a light-cone fermion. Nevertheless the preceding construcion is useful since it allowed us to isolate the $\mathcal{J}_{2}$ contribution to the spectrum $(\mu+v)^{2} / k$.

The right-moving part of the spectrum is somewhat simpler since there are no superpartners. This means that we can repeat our construction above and the eigenvalue of the $\bar{L}_{0}$ operator is simply obtained by adding to the dimension in Eq. (D.18) the contribution of the $\bar{J}^{2}$ operator and of some $U$ (1) coming from the gauge sector:

$$
\bar{\Delta}\left(\bar{\Phi}_{j \bar{\mu} \bar{n}}(\bar{z})\right)=-\frac{j(j+1)}{k}-\frac{\bar{\mu}^{2}}{k+2}+\left\{\frac{\bar{\mu}^{2}}{k+2}+\frac{1}{k_{g}}\left(\bar{n}+\frac{\bar{a}}{2}\right)^{2}\right\},
$$

where again $\bar{n} \in \mathbb{N}$ and $\bar{a} \in \mathbb{Z}_{2}$ depending on the sector. 



\section{Bibliography}

[ABEN89] I. Antoniadis, C. Bachas, J. R. Ellis and D. V. Nanopoulos, AN EXPANDING UNIVERSE IN STRING THEORY, Nucl. Phys. B328, 117-139 (1989).

[AGFM81] L. Alvarez-Gaume, D. Z. Freedman and S. Mukhi, THE BACKGROUND FIELD METHOD AND THE ULTRAVIOLET STRUCTURE OF THE SUPERSYMMETRIC NONLINEAR sigma MODEL, Ann. Phys. 134, 85 (1981).

[AGS00] R. Argurio, A. Giveon and A. Shomer, Superstring theory on AdS(3) $x$ $G / H$ and boundary $N=3$ superconformal symmetry, JHEP 04, 010 (2000), hep-th/0002104.

[AW03] M. Atiyah and E. Witten, M-theory dynamics on a manifold of $G(2)$ holonomy, Adv. Theor. Math. Phys. 6, 1-106 (2003), hep-th/0107177.

[BB02] G. Barnich and F. Brandt, Covariant theory of asymptotic symmetries, conservation laws and central charges, Nucl. Phys. B633, 3-82 (2002), hep-th/0111246.

$\left[\mathrm{BBH}^{+} 00\right]$ N. Berkovits, M. Bershadsky, T. Hauer, S. Zhukov and B. Zwiebach, Superstring theory on $A d S(2) \times S(2)$ as a coset supermanifold, Nucl. Phys. B567, 61-86 (2000), hep-th/9907200.

[BCZ85] E. Braaten, T. L. Curtright and C. K. Zachos, TORSION AND GEOMETROSTASIS IN NONLINEAR SIGMA MODELS, Nucl. Phys. B260, 630 (1985).

[BFHP96] J. Balog, P. Forgacs, Z. Horvath and L. Palla, Perturbative quantum (in)equivalence of dual sigma models in two-dimensions, Nucl. Phys. Proc. Suppl. 49, 16-26 (1996), hep-th/9601091.

[BFM $\left.{ }^{+} 98\right]$ J. Balog, P. Forgacs, N. Mohammedi, L. Palla and J. Schnittger, On quantum T-duality in sigma models, Nucl. Phys. B535, 461-482 (1998), hepth/9806068.

[BH58] A. Borel and F. Hirzebruch, Characteristic classes and homogeneous spaces. I, Amer. J. Math. 80, 458 (1958).

[BHTZ93] M. Banados, M. Henneaux, C. Teitelboim and J. Zanelli, Geometry of the (2+1) black hole, Phys. Rev. D48, 1506-1525 (1993), gr-qc/9302012.

[BJKZ96] P. Berglund, C. V. Johnson, S. Kachru and P. Zaugg, Heterotic Coset Models and (0,2) String Vacua, Nucl. Phys. B460, 252-298 (1996), hepth/9509170.

[BK94] C. Bachas and E. Kiritsis, Exact string theory instantons by dimensional reduction, Phys. Lett. B325, 103-114 (1994), hep-th/9311185. 
[BPS98] H. J. Boonstra, B. Peeters and K. Skenderis, Brane intersections, anti-de Sitter spacetimes and dual superconformal theories, Nucl. Phys. B533, 127-162 (1998), hep-th/9803231.

[BTZ92] M. Banados, C. Teitelboim and J. Zanelli, The Black hole in threedimensional space-time, Phys. Rev. Lett. 69, 1849-1851 (1992), hepth/9204099.

[Bus87] T. H. Buscher, A SYMMETRY OF THE STRING BACKGROUND FIELD EQUATIONS, Phys. Lett. B194, 59 (1987).

[Byr97] M. Byrd, The Geometry of SU(3), (1997), physics/9708015.

[C ${ }^{+}$98] P. Claus et al., Black holes and superconformal mechanics, Phys. Rev. Lett. 81, 4553-4556 (1998), hep-th/9804177.

[CCKM01] M. Cadoni, P. Carta, D. Klemm and S. Mignemi, AdS(2) gravity as conformally invariant mechanical system, Phys. Rev. D63, 125021 (2001), hep-th/0009185.

[CH94] O. Coussaert and M. Henneaux, Self-dual solutions of 2+1 Einstein gravity with a negative cosmological constant, (1994), hep-th/9407181.

[CHS91] J. Callan, Curtis G., J. A. Harvey and A. Strominger, World sheet approach to heterotic instantons and solitons, Nucl. Phys. B359, 611-634 (1991).

[CLW94] E. J. Copeland, A. Lahiri and D. Wands, Low-energy effective string cosmology, Phys. Rev. D50, 4868-4880 (1994), hep-th/9406216.

[CMPF85] J. Callan, Curtis G., E. J. Martinec, M. J. Perry and D. Friedan, STRINGS IN BACKGROUND FIELDS, Nucl. Phys. B262, 593 (1985).

[CS89] S. Chaudhuri and J. A. Schwartz, A CRITERION FOR INTEGRABLY MARGINAL OPERATORS, Phys. Lett. B219, 291 (1989).

[DFG ${ }^{+}$02] O. DeWolfe, D. Z. Freedman, S. S. Gubser, G. T. Horowitz and I. Mitra, Stability of $A d S(p) \times M(q)$ compactifications without supersymmetry, Phys. Rev. D65, 064033 (2002), hep-th/0105047.

[DFS03] N. Drukker, B. Fiol and J. Simon, Goedel's universe in a supertube shroud, Phys. Rev. Lett. 91, 231601 (2003), hep-th/0306057.

[DLP98] M. J. Duff, H. Lu and C. N. Pope, AdS(5) x S(5) untwisted, Nucl. Phys. B532, 181-209 (1998), hep-th/9803061.

[DLP99] M. J. Duff, H. Lu and C. N. Pope, AdS(3) $x S^{* * 3}$ (un)twisted and squashed, and an $O(2,2, Z)$ multiplet of dyonic strings, Nucl. Phys. B544, 145-180 (1999), hep-th/9807173.

[Dob87] V. K. Dobrev, CHARACTERS OF THE UNITARIZABLE HIGHEST WEIGHT MODULES OVER THE N=2 SUPERCONFORMAL ALGEBRAS, Phys. Lett. B186, 43 (1987).

[DOPS05] S. Detournay, D. Orlando, P. M. Petropoulos and P. Spindel, Threedimensional black holes from deformed anti de Sitter, JHEP 07, 072 (2005), hep-th/0504231.

[DVV92] R. Dijkgraaf, H. Verlinde and E. Verlinde, String propagation in a black hole geometry, Nucl. Phys. B371, 269-314 (1992).

[ES01] T. Eguchi and Y. Sugawara, CFT description of string theory compactified on non- compact manifolds with G(2) holonomy, Phys. Lett. B519, 149158 (2001), hep-th/0108091. 
[ESY03] T. Eguchi, Y. Sugawara and S. Yamaguchi, Supercoset CFT's for string theories on non-compact special holonomy manifolds, Nucl. Phys. B657, 3-52 (2003), hep-th/0301164.

[F9̈4] S. Förste, A Truly marginal deformation of $S L(2, R)$ in a null direction, Phys. Lett. B338, 36-39 (1994), hep-th/9407198.

[FKS95] S. Ferrara, R. Kallosh and A. Strominger, $N=2$ extremal black holes, Phys. Rev. D52, 5412-5416 (1995), hep-th/9508072.

[FR03] S. Förste and D. Roggenkamp, Current current deformations of conformal field theories, and WZW models, JHEP 05, 071 (2003), hep-th/0304234.

[Fri80] D. Friedan, NONLINEAR MODELS IN TWO epsilon DIMENSIONS, Phys. Rev. Lett. 45, 1057 (1980).

[Fri85] D. H. Friedan, NONLINEAR MODELS IN TWO + epsilon DIMENSIONS, Ann. Phys. 163, 318 (1985).

[Gaw91] K. Gawedzki, Noncompact WZW conformal field theories, (1991), hepth/9110076.

[Gep87] D. Gepner, NEW CONFORMAL FIELD THEORIES ASSOCIATED WITH LIE ALGEBRAS AND THEIR PARTITION FUNCTIONS, Nucl. Phys. B290, 10 (1987).

[Gep88] D. Gepner, SPACE-TIME SUPERSYMMETRY IN COMPACTIFIED STRING THEORY AND SUPERCONFORMAL MODELS, Nucl. Phys. B296, 757 (1988).

[Ger95] D. Gershon, Exact solutions of four-dimensional black holes in string theory, Phys. Rev. D51, 4387-4393 (1995), hep-th/9202005.

[GHMS03] M. Gutperle, M. Headrick, S. Minwalla and V. Schomerus, Space-time energy decreases under world-sheet RG flow, JHEP 01, 073 (2003), hepth/0211063.

[GHR84] J. Gates, S. J., C. M. Hull and M. Rocek, TWISTED MULTIPLETS AND NEW SUPERSYMMETRIC NONLINEAR sigma MODELS, Nucl. Phys. B248, 157 (1984).

[GIS02] G. W. Gibbons, D. Ida and T. Shiromizu, Uniqueness and non-uniqueness of static black holes in higher dimensions, Phys. Rev. Lett. 89, 041101 (2002), hep-th/0206049.

[GK94] A. Giveon and E. Kiritsis, Axial vector duality as a gauge symmetry and topology change in string theory, Nucl. Phys. B411, 487-508 (1994), hepth/9303016.

[GK98] S. Gnutzmann and M. Kuś, Coherent states and the classical limit on irreducible SU (3) representations, J.Phys. A 31, 9871 (1998).

[GK99] A. Giveon and D. Kutasov, Little string theory in a double scaling limit, JHEP 10, 034 (1999), hep-th/9909110.

[GKP99] A. Giveon, D. Kutasov and O. Pelc, Holography for non-critical superstrings, JHEP 10, 035 (1999), hep-th/9907178.

[GKPS03] A. Giveon, A. Konechny, A. Pakman and A. Sever, Type 0 strings in a 2-d black hole, JHEP 10, 025 (2003), hep-th/0309056.

[GL93] R. Gregory and R. Laflamme, Black strings and p-branes are unstable, Phys. Rev. Lett. 70, 2837-2840 (1993), hep-th/9301052. 
[GP94] D. S. Goldwirth and M. J. Perry, String dominated cosmology, Phys. Rev. D49, 5019-5025 (1994), hep-th/9308023.

[GQ87] D. Gepner and Z.-a. Qiu, MODULAR INVARIANT PARTITION FUNCTIONS FOR PARAFERMIONIC FIELD THEORIES, Nucl. Phys. B285, 423 (1987).

[GT99] G. W. Gibbons and P. K. Townsend, Black holes and Calogero models, Phys. Lett. B454, 187-192 (1999), hep-th/9812034.

[Heu98] M. Heusler, Stationary black holes: Uniqueness and beyond, Living Rev. Rel. 1, 6 (1998).

[HH92] J. H. Horne and G. T. Horowitz, Exact black string solutions in threedimensions, Nucl. Phys. B368, 444-462 (1992), hep-th/9108001.

[HHS92] J. H. Horne, G. T. Horowitz and A. R. Steif, An Equivalence between momentum and charge in string theory, Phys. Rev. Lett. 68, 568-571 (1992), hep-th/9110065.

[HKV02] S. Hemming and E. Keski-Vakkuri, The spectrum of strings on BTZ black holes and spectral flow in the SL(2,R) WZW model, Nucl. Phys. B626, 363-376 (2002), hep-th/0110252.

[Hor92] P. Horava, Some exact solutions of string theory in four-dimensions and five-dimensions, Phys. Lett. B278, 101-110 (1992), hep-th/9110067.

[HS93] S. F. Hassan and A. Sen, Marginal deformations of WZNW and coset models from $O(d, d)$ transformation, Nucl. Phys. B405, 143-165 (1993), hep-th/9210121.

[HT87] C. M. Hull and P. K. Townsend, THE TWO LOOP BETA FUNCTION FOR sigma MODELS WITH TORSION, Phys. Lett. B191, 115 (1987).

[HT95] G. T. Horowitz and A. A. Tseytlin, A New class of exact solutions in string theory, Phys. Rev. D51, 2896-2917 (1995), hep-th/9409021.

[HW93] G. T. Horowitz and D. L. Welch, Exact three-dimensional black holes in string theory, Phys. Rev. Lett. 71, 328-331 (1993), hep-th/9302126.

[IKOP05a] D. Israël, C. Kounnas, D. Orlando and P. M. Petropoulos, Electric / magnetic deformations of $S^{* *} 3$ and $A d S(3)$, and geometric cosets, Fortsch. Phys. 53, 73-104 (2005), hep-th/0405213.

[IKOP05b] D. Israel, C. Kounnas, D. Orlando and P. M. Petropoulos, Heterotic strings on homogeneous spaces, Fortsch. Phys. 53, 1030-1071 (2005), hepth/0412220.

[IKP03] D. Israël, C. Kounnas and M. P. Petropoulos, Superstrings on NS5 backgrounds, deformed AdS(3) and holography, JHEP 10, 028 (2003), hepth/0306053.

[IKPT04] D. Israël, C. Kounnas, A. Pakman and J. Troost, The partition function of the supersymmetric two-dimensional black hole and little string theory, JHEP 06, 033 (2004), hep-th/0403237.

[Isr67] W. Israel, Event horizons in static vacuum space-times, Phys. Rev. 164, 1776-1779 (1967).

[Isr04] D. Israël, Quantization of heterotic strings in a Gödel/anti de Sitter spacetime and chronology protection, JHEP 01, 042 (2004), hep-th/0310158. 
[Joh95] C. V. Johnson, Heterotic Coset Models, Mod. Phys. Lett. A10, 549-560 (1995), hep-th/9409062.

[Kir88] E. Kiritsis, CHARACTER FORMULAE AND THE STRUCTURE OF THE REPRESENTATIONS OF THE N=1, N=2 SUPERCONFORMAL ALGEBRAS, Int. J. Mod. Phys. A3, 1871 (1988).

[KK95] E. Kiritsis and C. Kounnas, Infrared behavior of closed superstrings in strong magnetic and gravitational fields, Nucl. Phys. B456, 699-731 (1995), hep-th/9508078.

[KKPR03] E. Kiritsis, C. Kounnas, P. M. Petropoulos and J. Rizos, Five-brane configurations, conformal field theories and the strong-coupling problem, (2003), hep-th/0312300.

[KLL99] D. Kutasov, F. Larsen and R. G. Leigh, String theory in magnetic monopole backgrounds, Nucl. Phys. B550, 183-213 (1999), hepth/9812027.

[KN69] S. Kobayashi and K. Nomizu, Foundations of Differential Geometry, Wyley, 1969.

[KPR91] C. Kounnas, M. Porrati and B. Rostand, ON N=4 extended superLiouville theory, Phys. Lett. B258, 61-69 (1991).

[KS89a] Y. Kazama and H. Suzuki, CHARACTERIZATION OF N=2 SUPERCONFORMAL MODELS GENERATED BY COSET SPACE METHOD, Phys. Lett. B216, 112 (1989).

[KS89b] Y. Kazama and H. Suzuki, NEW N=2 SUPERCONFORMAL FIELD THEORIES AND SUPERSTRING COMPACTIFICATION, Nucl. Phys. B321, 232 (1989).

[KT94] C. Klimcik and A. A. Tseytlin, Exact four-dimensional string solutions and Toda like sigma models from 'null gauged' WZNW theories, Nucl. Phys. B424, 71-96 (1994), hep-th/9402120.

[KT00] K.-I. Kondo and Y. Taira, Non-Abelian Stokes theorem and quark confinement in $S U(N)$ Yang-Mills gauge theory, Prog. Theor. Phys. 104, 11891265 (2000), hep-th/9911242.

[KZ84] V. G. Knizhnik and A. B. Zamolodchikov, Current algebra and WessZumino model in two dimensions, Nucl. Phys. B247, 83-103 (1984).

[LP96] H. Lu and C. N. Pope, p-brane Solitons in Maximal Supergravities, Nucl. Phys. B465, 127-156 (1996), hep-th/9512012.

[LPS96] H. Lu, C. N. Pope and K. S. Stelle, Weyl Group Invariance and p-brane Multiplets, Nucl. Phys. B476, 89-117 (1996), hep-th/9602140.

[LS92] H. Leutwyler and M. A. Shifman, Perturbation theory in the WessZumino-Novikov-Witten model, Int. J. Mod. Phys. A7, 795-842 (1992).

[Ma198] J. M. Maldacena, The large $N$ limit of superconformal field theories and supergravity, Adv. Theor. Math. Phys. 2, 231-252 (1998), hep-th/9711200.

[Mat87] Y. Matsuo, CHARACTER FORMULA OF C < 1 UNITARY REPRESENTATION OF N=2 SUPERCONFORMAL ALGEBRA, Prog. Theor. Phys. 77, 793 (1987).

[MHS88] F. Mueller-Hoissen and R. Stuckl, COSET SPACES AND TENDIMENSIONAL UNIFIED THEORIES, Class. Quant. Grav. 5, 27 (1988). 
[MN01] J. M. Maldacena and C. Nunez, Supergravity description of field theories on curved manifolds and a no go theorem, Int. J. Mod. Phys. A16, 822-855 (2001), hep-th/0007018.

[MO01] J. M. Maldacena and H. Ooguri, Strings in AdS(3) and SL(2,R) WZW model. I, J. Math. Phys. 42, 2929-2960 (2001), hep-th/0001053.

[Nak] M. Nakahara, Geometry, topology and physics, Bristol, UK: Hilger (1990) 505 p. (Graduate student series in physics).

[NS98] M. Natsuume and Y. Satoh, String theory on three dimensional black holes, Int. J. Mod. Phys. A13, 1229-1262 (1998), hep-th/9611041.

[OPS06] D. Orlando, P. M. Petropoulos and K. Sfetsos, Renormalization-group flows and charge transmutation in string theory, Fortsch. Phys. 54, 453461 (2006), hep-th/0512086.

[Orl05a] D. Orlando, $\operatorname{AdS}(2) \times S^{* * 2}$ as an exact heterotic string background, (2005), hep-th/0502213, Talk given at NATO Advanced Study Institute and EC Summer School on String Theory: From Gauge Interactions to Cosmology, Cargese, France, 7-19 Jun 2004.

[Orl05b] D. Orlando, Coset models and D-branes in group manifolds, (2005), hepth/0511210.

[Osb90] H. Osborn, GENERAL BOSONIC sigma MODELS AND STRING EFFECTIVE ACTIONS, Ann. Phys. 200, 1 (1990).

[Per87] A. M. Perelomov, CHIRAL MODELS: GEOMETRICAL ASPECTS, Phys. Rept. 146, 135-213 (1987).

[Pet90] P. M. S. Petropoulos, COMMENTS ON SU(1,1) STRING THEORY, Phys. Lett. B236, 151 (1990).

[Pol88] A. M. Polyakov, Two-dimensional quantum gravity: Superconductivity at high T/c, (1988), In *Les Houches 1988, Proceedings, Fields, strings and critical phenomena* 305-368.

[PW83] A. M. Polyakov and P. B. Wiegmann, Theory of nonabelian Goldstone bosons in two dimensions, Phys. Lett. B131, 121-126 (1983).

[PZT00] L. A. Pando Zayas and A. A. Tseytlin, Conformal sigma models for a class of $T(p, q)$ spaces, Class. Quant. Grav. 17, 5125-5131 (2000), hepth/0007086.

[QS03] T. Quella and V. Schomerus, Asymmetric cosets, JHEP 02, 030 (2003), hep-th/0212119.

[RS98] M. Rooman and P. Spindel, Goedel metric as a squashed anti-de Sitter geometry, Class. Quant. Grav. 15, 3241-3249 (1998), gr-qc/9804027.

[RT83] M. J. Reboucas and J. Tiomno, ON THE HOMOGENEITY OF RIEMANNIAN SPACE-TIMES OF GODEL TYPE, Phys. Rev. D28, 1251-1264 (1983).

[RY87] F. Ravanini and S.-K. Yang, MODULAR INVARIANCE IN N=2 SUPERCONFORMAL FIELD THEORIES, Phys. Lett. B195, 202 (1987).

[Sfe93] K. Sfetsos, Conformally exact results for $S L(2, R) \times S O(1,1)(d-2) / S O(1,1)$ coset models, Nucl. Phys. B389, 424-444 (1993), hep-th/9206048.

[Sfe98] K. Sfetsos, Poisson-Lie T-duality beyond the classical level and the renormalization group, Phys. Lett. B432, 365-375 (1998), hep-th/9803019. 
[Sfe99] K. Sfetsos, Duality-invariant class of two-dimensional field theories, Nucl. Phys. B561, 316-340 (1999), hep-th/9904188.

[Som68] C. M. Sommerfield, Currents as dynamical variables, Phys. Rev. 176, 2019-2025 (1968).

[SS06] A. Saltman and E. Silverstein, A new handle on de Sitter compactifications, JHEP 01, 139 (2006), hep-th/0411271.

[Ste98] K. S. Stelle, BPS branes in supergravity, (1998), hep-th/9803116.

[Sug68] H. Sugawara, A Field theory of currents, Phys. Rev. 170, 1659-1662 (1968).

[Tes99] J. Teschner, On structure constants and fusion rules in the $S L(2, C) / S U(2)$ WZNW model, Nucl. Phys. B546, 390-422 (1999), hep-th/9712256.

[Tse92a] A. A. Tseytlin, Cosmological solutions with dilaton and maximally symmetric space in string theory, Int. J. Mod. Phys. D1, 223-245 (1992), hepth/9203033.

[Tse92b] A. A. Tseytlin, Dilaton, winding modes and cosmological solutions, Class. Quant. Grav. 9, 979-1000 (1992), hep-th/9112004.

[Tse93] A. A. Tseytlin, Effective action of gauged WZW model and exact string solutions, Nucl. Phys. B399, 601-622 (1993), hep-th/9301015.

[Tse94] A. A. Tseytlin, Conformal sigma models corresponding to gauged WessZumino- Witten theories, Nucl. Phys. B411, 509-558 (1994), hepth/9302083.

[Tse95] A. A. Tseytlin, Exact solutions of closed string theory, Class. Quant. Grav. 12, 2365-2410 (1995), hep-th/9505052.

[Ver04] H. Verlinde, Superstrings on $A d S(2)$ and superconformal matrix quantum mechanics, (2004), hep-th/0403024.

[VK91] N. J. Vilenkin and A. Klimyk, Representation of Lie groups and special functions, Kluwer Academic Publishers, Dordrecht, 1991.

[Wit84] E. Witten, Nonabelian bosonization in two dimensions, Commun. Math. Phys. 92, 455-472 (1984).

[Wit91] E. Witten, On string theory and black holes, Phys. Rev. D44, 314-324 (1991).

[You99] D. Youm, Black holes and solitons in string theory, Phys. Rept. 316, 1-232 (1999), hep-th/9710046.

[Zam86] A. B. Zamolodchikov, 'IRREVERSIBILITY' OF THE FLUX OF THE RENORMALIZATION GROUP IN A 2-D FIELD THEORY, JETP Lett. 43, 730-732 (1986). 